Florida International University FIU Digital Commons

3-15-2007

\title{
An integrated framework for ensuring the quality of software design
}

\author{
Yujian $\mathrm{Fu}$ \\ Florida International University
}

DOI: $10.25148 /$ etd.FI15101455

Follow this and additional works at: https://digitalcommons.fiu.edu/etd

Part of the Computer Sciences Commons

\section{Recommended Citation}

$\mathrm{Fu}$, Yujian, "An integrated framework for ensuring the quality of software design" (2007). FIU Electronic Theses and Dissertations. 3612. https://digitalcommons.fiu.edu/etd/3612

This work is brought to you for free and open access by the University Graduate School at FIU Digital Commons. It has been accepted for inclusion in FIU Electronic Theses and Dissertations by an authorized administrator of FIU Digital Commons. For more information, please contact dcc@fiu.edu. 
FLORIDA INTERNATIONAL UNIVERSITY

Miami, Florida

AN INTEGRATED FRAMEWORK FOR ENSURING THE QUALITY OF SOFTWARE DESIGN

A dissertation submitted in partial fulfillment of the

requirements for the degree of

DOCTOR OF PHILOSOPHY

in

COMPUTER SCIENCE

by

Yujian Fu

2007 
To: Dean Vish Prasad

College of Engineering and Computing

This dissertation, written by Yujian Fu, and entitled An Integrated Framework for Ensuring the Quality of Software Design, having been approved in respect to style and intellectual content, is referred to you for judgment.

We have read this dissertation and recommend that it be approved.

Shu-Ching Chen

Peter J. Clarke

Yi Deng

Jie $\mathrm{Mi}$

Xudong He, Major Professor

Date of Defense: March 15, 2007

The dissertation of Yujian Fu is approved.

Dean Vish Prasad College of Engineering and Computing

Dean George Walker University Graduate School

Florida International University, 2007 
(C) Copyright 2007 by Yujian Fu

All rights reserved. 


\section{DEDICATION}

To my parents, my husband and my son. 


\section{ACKNOWLEDGMENTS}

I almost cannot believe that this long thesis marathon is nearing the end, and I am greatly indebted to many people who helped me get started, pointed in the right direction, kept me on track, and supported along the way, and without whom I would have never made it.

First of all, I would like to thank my advisor, Xudong $\mathrm{He}$, who provided the invaluable guidance and financial support throughout my entire stay at Florida International University. He has taught me innumerable things one needs as a researcher from writing papers. I am especially grateful for his inspiration for the instructing and correcting me to do certain parts of my work, which, as I discovered later, turned out to be absolutely necessary, and without them my work would be incomplete.

Thanks to all my committee members for taking their time to serve on the committee, Shu-Ching Chen, Peter J Clarke, Yi Deng, Raimund K. Ege, and Jie Mi and for the feedback they have provided. Most of all, I appreciate the detailed discussions with Ken McMillan about SMV, the theory behind it and practical issues, and many interesting subtleties in the abstraction mechanism and its implementation.

Many thanks to the members of our research group, it has been my great pleasure to work with so many bright and talented people. Zhijiang Dong has significantly contributed to the development of the tool SAM Parser, and in particular, wrote the logic server that pass data from core Maude to Java construct and all of the property verification of the system, which was crucial to my finishing the implementation in time. And thanks to the other group members for a lot of inspiring and insightful discussions: Gonzalo Argote, Weixiang Sun, Miaohua Xu, and everyone else for both research and life concerning in Miami during past years.

Furthermore, I cannot imagine surviving the tense life of a graduate student within gloomy walls of my office without encouragement of my parents, Xuebo Fu and Xiuyun Yao, helping me stay upbeat, letting me vent all my thesis bitterness, and assisting with many small problems. 
And finally, most of my gratitude goes to my dear husband, Zhijiang Dong, for his unsurpassed gift of persuasiveness, great talent of ferreting out information, and amazing faith in what I always thought as impossible. Without him, I would have not even come to FIU in the first place. With his help and support, I have made it through the application process, moved to Miami, stayed sane and in working order for all six years, and finally is about to receive my degree from FIU something I have never even dreamed of before I met him. I cannot thank him enough for being wonderfully helpful.

This work is supported in part by NSF under grant HRD-0317692, and by NASA under grant NAG 2-1440. I also appreciate the financial support from the University Graduate School, Florida International University in the form of Dissertation Year Fellowship. 


\section{ABSTRACT OF THE DISSERTATION}

\section{AN INTEGRATED FRAMEWORK FOR ENSURING THE QUALITY OF SOFTWARE \\ DESIGN}

by

Yujian Fu

Florida International University, 2007

Miami, Florida

Professor Xudong He, Major Professor

Software development is an extremely complex process, during which human errors are introduced and result in faulty software systems. It is highly desirable and important that these errors can be prevented and detected as early as possible. Software architecture design is a high-level system description, which embodies many system features and properties that are eventually implemented in the final operational system. Therefore, methods for modeling and analyzing software architecture descriptions can help prevent and reveal human errors and thus improve software quality. Furthermore, if an analyzed software architecture description can be used to derive a partial software implementation, especially when the derivation can be automated, significant benefits can be gained with regard to both the system quality and productivity.

This dissertation proposes a framework for an integrated analysis on both of the design and implementation. To ensure the desirable properties of the architecture model, we apply formal verification by using the model checking technique. To ensure the desirable properties of the implementation, we develop a methodology and the associated tool to translate an architecture specification into an implementation written in the combination of ArchJava/Java/AspectJ programming languages. The translation is semi-automatic so that many manual programming errors can be prevented. Furthermore, the translation inserting monitoring code into the implementation such that runtime verification can be performed, this provides additional assurance for the quality of the implementation. Moreover, validations 
for the translations from architecture model to program are provided. Finally, several case studies are experimented and presented. 


\section{TABLE OF CONTENTS}

CHAPTER

PAGE

1 Introduction . ......................... 1

1.1 Assurance of the Correctness of Software Systems . . . . . . . . . . . 2

1.2 Necessity of the Integration of Model Checking and Runtime Analysis . . . 4

1.3 Contribution . . . . . . . . . . . . . . . . . . 6

1.4 Chapter Organization of Dissertation . . . . . . . . . . . . . . . 9

2 Related Work . . . . . . . . . . . . . . . . . . . . . . . 11

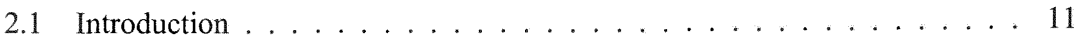

2.2 Analysis of Systems . . . . . . . . . . . . . . . . . . 11

2.2.1 Analysis on the Model of Systems . . . . . . . . . . . . 11

2.2.2 Analysis of Implementation . . . . . . . . . . . . . . 23

2.3 Translation Correctness . . . . . . . . . . . . . . . . . . . . . . . . . . . 29

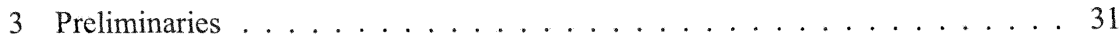

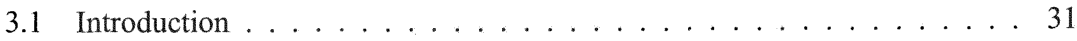

3.2 SAM - Software Architecture Model . . . . . . . . . . . . . . . 31

$3.2 .1 \quad$ Petri Nets . . . . . . . . . . . . . . . . . . 33

3.2 .2 Temporal Logic . . . . . . . . . . . . . . . . . . 35

3.3 Maude . . . . . . . . . . . . . . . . . . . . . 37

3.3 .1 Introduction . . . . . . . . . . . . 37

3.3.2 Rewriting Logic and Membership Logic . . . . . . . . . . . . 38

3.3.3 Functional Module \& System Module . . . . . . . . . . . . . . . . 39

3.3.4 Maude Model Checking . . . . . . . . . . . . . . . . . . 41

3.4 ArchJava Programming Language . . . . . . . . . . . . . . . 43

3.4.1 Components, Ports and Architecture Composition . . . . . . . . . 43

3.4.2 Connector Abstractions in ArchJava . . . . . . . . . . . . . 46

3.5 Aspect Oriented Programming Languages . . . . . . . . . . . . . . . 46

3.6 Summary . . . . . . . . . . . . . . . . . . . . . . . . . . 48

4 An Overview of Integrated Framework . . . . . . . . . . . . . . . . . . . . 49

4.1 Introduction . . . . . . . . . . . . . . . . . . . . . 49

4.2 The Integrated Framework . . . . . . . . . . . . . . . . 51

4.2 .1 Input Layer . . . . . . . . . . . . . . . . . . . . 53

4.2 .2 Translation Phase . . . . . . . . . . . . . . . . . . 54

4.2 .3 Translation Validation . . . . . . . . . . . . . 55

4.2 .4 Verification Phase . . . . . . . . . . . . . . . . . . 55

5 Verification and Validation in the Design $\ldots \ldots \ldots \ldots$

5.1 Introduction . . . . . . . . . . . . . . . 56

5.2 Related Work . . . . . . . . . . . . . . . . . . . . . 57

5.3 Software Architecture Verification \& Analysis . . . . . . . . . . . . . 57

5.3.1 Software Architecture Verification \& Analysis Method . . . . . . . 57

5.3.2 Software Architecture Perspectives . . . . . . . . . . . . . . 59 
5.4 Translation from SAM to Maude . . . . . . . . . . . . . . . 66

5.5 Summary . . . . . . . . . . . . . . . . . . 70

6 Verification \& Validation in Implementation . . . . . . . . . . . . . . 71

6.1 Introduction . . . . . . . . . . . . . . . 71

6.2 Related Works . . . . . . . . . . . . . . . . . . . . . 71

6.3 System Architecture . . . . . . . . . . . . . . . . . . . . 73

6.4 Translation from SAM to Java Construct . . . . . . . . . . . . . . . . 74

6.4.1 Architecture Structure Translation . . . . . . . . . . . . . . . 74

6.4.2 Behavior Model Translation . . . . . . . . . . . . . . . 77

6.4 .3 Property Specification . . . . . . . . . . . . . . . 77

6.4 .4 Tool Support . . . . . . . . . . . . . . . . . . . . . . . . . . . . . . 79

6.5 Runtime Verification on SAM . . . . . . . . . . . . . . . 80

6.5.1 Propositional Linear Temporal Logic Formulae . . . . . . . . . . 80

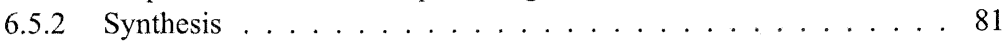

6.6 Summary . . . . . . . . . . . . . . . . . 82

7 Translation Validation $\ldots \ldots \ldots \ldots$. . . . . . . . . 86

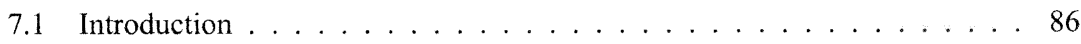

7.1.1 Translation Correctness . . . . . . . . . . . . . . . . . . . 87

7.2 Related Work . . . . . . . . . . . . . . . . . . . 88

7.3 Validation of Translation from SAM to Maude . . . . . . . . . . 90

7.3.1 Rewriting Theories and Deduction . . . . . . . . . . . 90

7.3 .2 A theory for Petri nets . . . . . . . . . . . . . . . 994

7.3.3 An interleaving correspondence . . . . . . . . . . 995

7.4 Validation of Translation from SAM to Java Construct . . . . . . . . . 98

7.4.1 Interleaving Semantics of Restricted PrT nets . . . . . . . . . 100

7.4.2 Sequential Semantics of Simplified Java Construct . . . . . . . . . 104

7.4 .3 Mapping Relation . . . . . . . . . . . . . . . . . . . . . . 109

7.4 .4 Correspondence . . . . . . . . . . . . . . . . . . 111

7.5 Discussion . . . . . . . . . . . . . . . . . . . . 112

7.5.1 Interleaving Semantics vs. Causal Semantics . . . . . . . . 112

7.5 .2 Granularity . . . . . . . . . . . . . . . . . . . 113

7.5.3 State-based vs. action-based . . . . . . . . . . . . 114

7.6 Summary . . . . . . . . . . . . . . . . . . . 115

8 Case Studies . . . . . . . . . . . . . . . . . . . . . . . . . 121

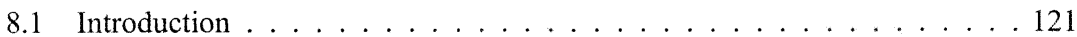

8.2 Coffee Machine . . . . . . . . . . . . . . . . . . . . . 121

8.2 .1 Model Checking . . . . . . . . . . . . . . 125

8.2 .2 Runtime Verification . . . . . . . . . . . . . . . . . . 126

8.2 .3 Discussion . . . . . . . . . . . . . . . . . . 128

8.3 Network Security Protocol Under Attack . . . . . . . . . . . . . . . . . . 129

8.3.1 Scenario Introduction . . . . . . . . . . . . . . . . . . . . . 129

8.3.2 SAM Model of Network Security Protocol Under Attack . . . . . . 130

8.3.3 System Properties . . . . . . . . . . . . . . . . . . . . 132 
8.3 .4 Model Checking . . . . . . . . . . . . . . . . . . 133

8.3 .5 Runtime Verification . . . . . . . . . . . . . . . . . 133

8.4 Online Shopping . . . . . . . . . . . . . . . . . . . . . . . 134

8.4 .1 Scenario Introduction . . . . . . . . . . . . . . . . . 134

8.4 .2 SAM Model of Online Shopping System . . . . . . . . . 135

8.4 .3 System Properties . . . . . . . . . . . . . . . 137

8.4 .4 Model Checking . . . . . . . . . . . . . . . . . . . . . 140

8.4.5 Runtime Verification . . . . . . . . . . . . . . . . . . . 140

8.5 Cruise Control System . . . . . . . . . . . . . . . . . . . . 141

8.5 .1 Scenario Introduction . . . . . . . . . . . . . . . . . . 141

8.5 .2 SAM Model of CC System . . . . . . . . . . . . . . . . 142

8.5.3 Properties of the System . . . . . . . . . . . . . . . . . 143

8.5 .4 Runtime Verification . . . . . . . . . . . . . . . . . . . . . 144

8.6 Service Oriented Software Architecture Model (SO-SAM) . . . . . . . 145

8.6.1 SO-SAM Specification . . . . . . . . . . . . . . . . . 147

8.6 .2 Service Composition . . . . . . . . . . . . . . . 151

8.6.3 A Case Study on the SO-SAM Model . . . . . . . . . . . . 156

8.7 Application on UML Architecture Description of Web Systems . . . . . . 156

8.7.1 An Image Processing Example . . . . . . . . . . . . . . . . 159

8.7.2 Transformation From Component \& Connector View . . . . . . . 163

8.7.3 Validation of the Approach . . . . . . . . . . . . 173

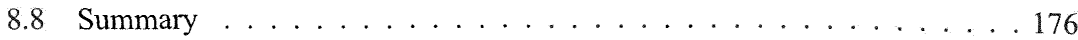

9 Conclusion . . . . . . . . . . . . . . . . . . . . . 180

9.1 Summary . . . . . . . . . . . . . . . . . . 180

9.2 Future Work . . . . . . . . . . . . . . . . . . . . . . . 182

LIST OF REFERENCES . . . . . . . . . . . . . . . . . . . . 183

APPENDICES . . . . . . . . . . . . . . . . 200

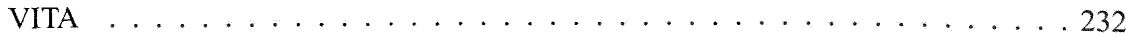




\section{LIST OF TABLES}

TABLE

PAGE

1 Event trace semantics of TL formula . . . . . . . . . . . . . . 19

2 Summary of Implementation Analysis . . . . . . . . . . . . . . . . . . . 29

3 Correspondence between Reference Frame and Integrated Framework . . . 52

4 Mapping relation between architecture structure and ArchJava . . . . . . 75

5 Component Class definition in ArchJava . . . . . . . . . . . . . . 76

6 Mapping relation between Syntax of Petri net and Java . . . . . . . . 78

$7 \quad$ Inference Rules . . . . . . . . . . . . . . . . . . 92

8 COORE Syntax of Sequential Java Construct [170] . . . . . . . . . . 106

9 Expression Semantics [169] . . . . . . . . . . . . . . 107

10 Operational Semantics of COORE [169] . . . . . . . . . . . . . 117

11 Communication Trace . . . . . . . . . . . . . . 118

12 Expression Semantics [169] . . . . . . . . . . . . . . 119

13 A Mapping Relation of Elements in Component/Connector of SAM model . 119

14 Summary of Generated Files . . . . . . . . . . . . . . . . 127

15 Relation between Size and Time of Coffee Machine Example . . . . . . . . 128

16 Statistic Data of Generated Files . . . . . . . . . . . . . . . 141

17 Relation between Size and Time of Online Shopping Example . . . . . . . 142

18 A Path of Executing Protocols RawData and DataPacket . . . . . . . . 174 


\section{LIST OF FIGURES}

FIGURE

PAGE

1 Three Areas of the Integrated Framework Addressed . . . . . . . . . . . 7

2 Summarization of the formal analysis methods of the software design . . . 24

3 A SAM Architecture Model . . . . . . . . . . . . . . . . 32

4 SAM Model of Coffee Machine . . . . . . . . . . . . . . . . . . 32

5 Behavior of a Subcomponent (CMInterface) in CoffeeMachine . . . . . . . 35

6 A Shorthand Example of ArchJava . . . . . . . . . . . . . . . . . 44

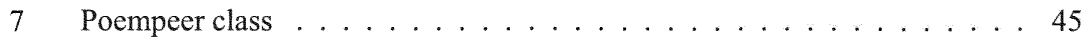

8 PoemSwap class . . . . . . . . . . . . . . . . . 46

9 An Example of AspectJ . . . . . . . . . . . . . . . . . 47

10 Reference Frame of the Idea . . . . . . . . . . . . . . . . . 49

11 Overview of the Integrated Framework . . . . . . . . . . . 51

12 Property Hierarchy . . . . . . . . . . . . . . . . . 62

13 An Overview of Our Methodology .................. 84

14 Generated Aspect for Composition ECompany . . . . . . . . . . . . . . 85

15 Visualization of 5 Inference Rules . . . . . . . . . . . . 93

16 An Architecture of Translation . . . . . . . . . . . . . . . . . . 99

17 The Proof Methodology of Translation to Java Construct . . . . . . . . . 100

18 SAM Model of Coffee Machine . . . . . . . . . . . . . . . . . 122

19 Behavior of Subcomponents - CoinHandler - in CoffeeMachine . . . . . . 123

20 Behavior of Subcomponents - BrewingFacility - in CoffeeMachine . . . . 124

21 Behavior of Subcomponents - CMInterface - in CoffeeMachine ... . . 125

22 SAM Architecture of STS with Intruder . . . . . . . . . . 131

23 Natural language description of electronic shopping System . . . . . . . . 135

24 SAM Architecture of online shopping scenario . . . . . . . . . . . . 136

25 SAM Model of Cruise Control System . . . . . . . . . . . . . . . 143

26 A General Service Oriented Architecture . . . . . . . . . . . . . . 152 
27 UML Extension for component and connector View . . . . . . . . . . 160

28 Structural Aspect of Component and Connector View . . . . . . . . . 161

29 Protocols in Component ImageProcessing . . . . . . . . . . . . 162

30 Behavior of Elements (UML) . . . . . . . . . . . . . . 163

31 Behavior of Elements (PrT Nets) . . . . . . . . . . . . . 178

32 ImageProcessing in SO-SAM . . . . . . . . . . . . . . . 179

33 SAM model of online shopping . . . . . . . . . . . . . . . 227

34 Behavior model of $U s e r$ component $\ldots \ldots \ldots \ldots \ldots$

35 Behavior model of Cart component . . . . . . . . . . . . . . . . 229

36 Behavior model of Warehouse component . . . . . . . . . . . . . . 229

37 Behavior model of Order component . . . . . . . . . . . . . 230

38 Behavior model of PaymentProcess component . . . . . . . . . 231

39 Behavior model of RetailerBank component . . . . . . . . . . . . . 231 


\section{CHAPTER 1 \\ INTRODUCTION}

This dissertation presents an integrated framework for the software quality assurance at the design level and its automated realization. Methods for proving the correctness the translation programs are also provided. This framework is part of the overall SAM [198] software development paradigm, which has been developed at FIU for the past 10 years and funded by several major NSF grants. In the SAM software development paradigm, a software system is formally modeled and specified at software architecture design level with subsequent refinements. The correctness of a formal SAM architecture design is formally verified. The translation from SAM architecture model to a program language of a formal verification tool is developed and the correctness is formally validated. Furthermore, this research extends existing research results on SAM through automated implementation of SAM architecture specifications. This prototype implementation provides additional means for ensuring system properties at implementation level using runtime verification, and can serve as a basis for production quality implementation. The translation method from a SAM architecture design consists of several parts: structure translation, behavior translation, and property mapping.

So this framework is not specific to one model but with general purpose. The formal verification technique of the software architecture specification is applied and correctness of translation is well established based on the well defined theory - rewriting theory [144]. A prototype implementation of the runtime checker of the framework on SAM, a software architecture description language, was developed. Structure and behavior of SAM model are translated into ArchJava and Java code respectively. System instrumentation and runtime monitor is implemented using AspectJ. The translation design demonstrates a systematical mapping from the model to its corresponding implementation. In addition, a 
correctness proof of the translated program in the implementation level is provided. This chapter describes the difficulties faced by the software engineering field with regard to quality, specifically correctness and the motivation for this work. This chapter concludes with contributions of this research to the field of software engineering in terms of increasing quality.

\subsection{Assurance of the Correctness of Software Systems}

When compared to software engineering's significance and broad application areas, the field's ability to provide correct and reliable products seems greatly lacking. This deficiency has become a great danger in today's world. Specifically, safety critical software systems such as airplane controllers or nuclear reactor controllers can devastate human lives and properties when they fail to behave correctly. There have been many disasters due to the incorrect execution of software systems [127], including the tragic accident of the Ariance 5 flight 501 [13]. The report "Information Technology Research: Investing in Our Future" from PITAC (President's Information Technology Advisory Committee) [16] pointed out that the software maintaining and testing are under the pressure of change.

In 2003, Tony Hoare proposed the "Grand Challenge" of the program verifier [102], a project that involved thousands of researchers for the next 15 to 20 years to build a verifying compiler that

"uses mathematical and logical reasoning to check that programs that it compiles." [102]

The fundamental problems are how to obtain and document the correctness specification of a program and how to fully automate the verification/checking of the documented aspects correctness. While accomplishing this goal now requires effort from "(almost) the entire research community" including theoretical researchers, compiler writers, tool builders, software developers and users, these two interrelated problems have been the major concern of the formal methods research community in the past several decades. This dissertation discusses the correctness assurance of software, which is one of the fundamental aspects of the "Grand challenge." 
Currently many methods and techniques have been developed to verify software systems in both design ([106, 115, 139]) and implementation level ( $[91,95,125,126])$. Among them model checking, a formal verification technique, is widely used in the design verification due to its highly automation. runtime analysis [95], a light-weighted formal verification technique, has been explored in the implementation level. Verification of software architecture specification is to check the software architecture model (generally formally specified) against the system properties. At present, various of meaningful architectural specifications make it feasible to determine the properties of a software system and enable more thorough and less costly testing. Unfortunately such specifications are rarely used in industrial. One of the reasons is that there is not a correspondence between a specification and the software itself. In other words, even if some properties can be verified in the system model through the endeavored efforts, the implemented code of the model after we shifted can still be messed up because of the inconsistency between model and implementation. At the same time, this consistency relationship is hard to be maintained without a systematic mapping strategy. Also, the properties of the implementation of the corresponding system model still need to be checked to validate the model at the implementation level. Furthermore, often software behavior and flaws are observable only during a program's execution, and even then may be invisible except under certain unusual conditions. Programs written in such circumstances frustrate attempts to create robust systems and are inherently fragile.

Thus, a scientifically sound approach to software development is required to be able to deal with meaningful and practical verification for consistency of specifications and implementations.

\subsection{Necessity of the Integration of Model Checking and Runtime Analysis}

For the past twenty years, formal verification methods have enlarged its domain of application as computing power has increased, so that formal verification can handle more than just toy examples. Testing has been a standard practice to ensure the correctness quality of software. However, formal verification and testing have limitations. Formal verification 
methods are performed before programs are put into the real environment. Thus these heavy-weighted formal methods are mostly concerned as static verification techniques. They have their own limitations in terms of effectiveness. I explain in the following.

Formal verification requires a formal software specification language. Temporal logic [171] is a formal specification language that extends propositional and first order logic with the timing span. With its accurate semantics temporal logic has been widely applied in the formal verification applications. There are some user-defined logics for its simple syntax and implementation environment [119]. However, there is a huge gap between a specification and the software itself. Model checking and theorem proving are two main formal methods used for the verification of software systems. Because of expert knowledgement, notations and less automation theorem proving are mainly used for the infinite system verification in that case model checking has less ability. Model checking is a technique building a finite system and a finite state model of a system and checking whether a given property holds in that model. Model-checking algorithms offer an exhaustive searching and automatic application to completely analyze the system. Counterexamples are provided when errors are found. However, model-checking may only verify systems expressed through state-based machines, it is more limited than theorem proving, and it suffers of the state explosion problem. Moreover, model-checking may be difficult to be applied, since it usually requires skills on formal specification languages.

Furthermore, because of the difficulty of using formalism and the limitation on the size of the model, formal verification is still not a feasible solution to obtain the correctness of the execution of whole software. Testing has been the prevalent choice for ensuring the execution of software. However, testing cannot give complete assurance of correct execution in the sense that exhaustive testing is infeasible [154]. Therefore, we cannot rely on only these two methods for obtaining reliability on the correct execution of software.

Compared to the above approaches, runtime verification is designed to ensure the runtime conformance of an execution trace of a system with its formal requirement specification. Runtime verification takes a program and a formal requirement specification as 
inputs. When a program is running, the execution of the program is checked against the formal requirement specification at runtime. The purpose of runtime formal analysis is to bridge the gap left by formal verification and testing. Formal verification attempts to guarantee that all possible executions of software yield correct results. In comparison, runtime formal analysis provides the correctness on the current execution of a program. While a program is running under the supervision of a monitor, we can make sure that so far the program has behaved correctly. Thus researchers can say that runtime verification is not complete. Furthermore, runtime verification is done after the program is released while formal verification has to be done before the product is released. Finally, formal verification is performed in the development stage so it is considered as verification technique while runtime analysis is performed after the product is released and conducted while the program is running so it is a post-development, monitoring technique.

Runtime formal analysis helps detect, identify and correct errors while the program is running, which is a great aid to the assurance of program correctness. There are many cases when users cannot detect errors because of the subtlety. For example, the Pentium floating point unit bug is so subtle that it could not be discovered for a long time. Even when errors are detected, it is hard to identify the source. For example, when a program crashes by Segmentation Fault on Unix, let us just guess there is incorrect pointer arithmetic in the program. However, we do not know the place and computation data where the Segmentation Fault happens. Furthermore, early detection of an error can lead an effectual meta-level activity, such as human intervention or automatic recovery action.

Considering the strong complementarity between those two worlds, I believe an integration between model checking and runtime analysis may provide an useful approach to verify large scale complex software systems.

Moreover, runtime verification at the design level is more significant than that in the implementation. The error detection is performed at an earlier stage is one of the most obvious advantage. First, the verification at the design level can check the implementation is consistent with its model. Although there is a fine model of a software system, when we 
we move to the implementation, various errors may be introduced due to the requirement of implementation of the program. Thus the runtime verification at the implementation level can provide more confidence for the developer and designer for the system analyst. Furthermore, some issues can only be observed in the execution. For instance, duplicate definition, overflow, file not found, and others, are all program errors exhibits only during program running. Finally, because of the state explosion of model checking, runtime verification is preferred to solve the scalability of the system. Although several heavyweighted formal verification methods are widely used in the verification of system model, runtime verification in the design level is still a very challengeable research area. The next chapter deals with most of the current research work and focuses on the program in the implementation level. Thus this is an open area for the runtime verification on the model of the systems. Even if one can show that the implementation is correct in terms of some properties, one cannot say the implementation follows the system design and system design correctness. This area can also bridge the gap between the design and implementation of the system development process.

\subsection{Contribution}

This dissertation addresses the following four areas of the integrated verification on SAM architecture model depicted in the Figure. 1,

1. Model checking at the design level

This dissertation discusses what properties model checking can verified based on the SAM architecture model. The model checker used in this dissertation is (core) Maude [47], which is a rewriting logic based high level reflective programming language with tool support. Because of its efficient rewriting engine and its metalanguage features, Maude turns out to be an excellent tool to create executable environments for various logics, theorem provers, and even programming languages. In this context, we identify following contributions. First, software architecture verification 


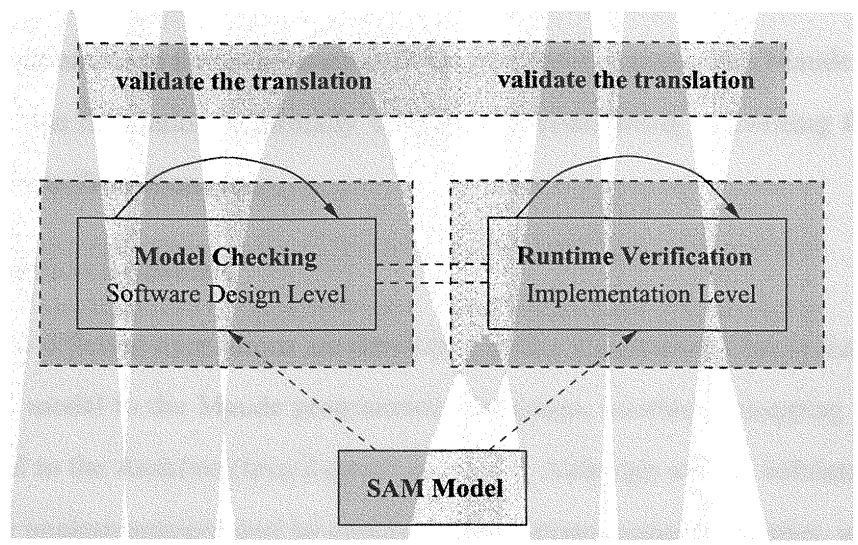

Figure 1: Three Areas of the Integrated Framework Addressed

and analysis methods in design level is developed, which is a general systematic stepwise approach that summarizes the formal analysis methods for the design model. Secondly, the instantiation of the method on the model checking tool (Maude) with several case studies reveal the necessity and flexibility of the method. Statistical data is collected and analyzed. Finally, the translation from SAM architecture model to the Maude programming language has been formally validated.

2. Runtime checking at the implementation level

The runtime verification architecture monitors and checks the execution of the target SAM program through a property based monitor extracted from the core Maude. Maude is used here to generate the finite automaton for a property. The runtime checker is generated from the finite automaton based on the algorithm proposed by Rosu [177]. The target SAM program is generated from the behavior model of SAM. The translation algorithm is needed to transform the SAM model to the input of runtime checker, a package of XML format file. In this context, we identify following contributions. First, in the development of prototype tool, SAM Parser, architecture structure translation is developed and realized using ArchJava. Second, properties 
transformation to finite automata is developed and extracted from Maude output. Finally, the translation is formally validated by establishing a rewriting theory for a Predicate Transition Net.

\section{Translation validations}

Two different translations are developed in this dissertation. One is mapping from SAM model to the Maude programming language, another is mapping from SAM model to the ArchJava/Java/AspectJ construct. Although we can validate the model at the implementation level by checking the program against properties, we still need to ensure the correctness of the translation at the implementation level - the program is the corresponding image to the model. Although we can validate the model at the design level by checking properties of the model using some model checker, we don't know the program written by the model checker programming language corresponding to the model after the translation, e.g., the correctness of translation at the design level is not validated yet.

The contribution in this context is presented as follows. First, a translation correctness is formally defined as a criteria for the target validation. To validate the translation from SAM to the ArchJava/Java/AspectJ construct, the correctness of the generated Java program is proved on the behavior model - Petri nets. Based on the implementation, the ArchJava/Java/AspectJ construct is compiled to Java program. After the careful analysis based on the simplification, a sequential object oriented program and its operational semantics are introduced to represent the Java program. After that, the definition of communication event is given based on the asynchronous invocation among objects and furthermore, the communication trace is defined as a sequence of communication events. Finally, I prove the consistency between the communication trace of the simplified object oriented program and the execution trace of the behavior model of SAM component/connector - a Predicate Transition Net. 
4. System modeling and analysis of Service Oriented Architecture (SOA)

SOAs will not scale without significant mechanization of service discovery, service adaptation, service composition, and service monitoring; as well as data, protocol, and process mediation. In this context, my contributions are: first extend SAM model to the service oriented architecture, as named SO-SAM, to formally analysis service composition, integration; secondly, apply the integrated framework on the SO-SAM model to validate the methodology on the SO-SAM model; finally, a case study is presented.

\subsection{Chapter Organization of Dissertation}

Chapter 2 describes the analysis methods for the correctness of the formal architecture specification in both design and implementation level. First, I classify the different analysis method used in the formal specifications: theorem proving vs. model checking. After that, a classification for the representation of the target program in the implementation level is given: abstract model vs. implementation. In this chapter, I evaluate related work, which helps the reader to understand the design rationale of the integrated framework.

Chapter 3 identifies fundamental knowledge used in this dissertation, including software architecture model (SAM) with Petri nets and temporal logic, ArchJava and Aspect oriented programming language. The last two are used in the runtime analysis and the implementation of the prototype tool SAM Parser.

Chapter 4 gives an overview of the integrated framework. This chapter includes the structure of the framework, how to analyze the system properties using this framework. This chapter is mainly from my previous work [56].

Chapter 5 introduces a software architecture verification and analysis method and applied it in the model checking of SAM model [77]. Moreover, a translation algorithm from SAM model to Maude programming language is presented. The translation algorithm is automatically implemented in the java program and integrated with the runtime verification code (Chapter 6). 
Chapter 6 focuses on the method and implementation of realizing the runtime analysis of SAM model in the implementation level. The translation algorithm is presented from SAM model to ArchJava/Java/AspectJ construct. The functional code and monitor code generation are discussed. The synthesis of a runtime checker is demonstrated. Finally, a prototype tool, SAM Parser is developed and presented. Details of the components of the prototype tool are described.

Chapter 7 discusses the validation approaches for the translations from SAM architecture model. Two translations are validated - from SAM to Maude programming language and to Java construct. Translation from SAM to Maude programming language is validated by establishing consistency between behavior model of SAM component/connector and rewriting theory. Translation from SAM to ArchJava/Java/AspectJ construct is constructed by establishing the consistency between behavior model of SAM component/connector and Java construct. The limitations of Petri nets are imposed on the second translation validation. Both are established on the translation correctness criteria.

Chapter 8 demonstrates the effectiveness of the integrated framework by illustrating case studies. The case studies include a coffee machine, a network protocol under attack scenario, an online shopping example, a cruise control system. An extension of SAM model to analyze the service oriented architecture is demonstrated. Finally, an application of the integrated framework on the UML architecture description is presented.

Chapter 9 summarizes and concludes the dissertation has achieved and points out future works. 


\section{CHAPTER 2 \\ RELATED WORK}

\subsection{Introduction}

In this chapter, we first review analysis methods for checking the correctness of programs (see Section 2.2). Two groups are discussed: methods for a model of a program and methods for an implementation of a program. After that we introduce the correctness proof of translation based on the design and implementation level (see Section 2.3).

\subsection{Analysis of Systems}

In this section we discuss the analysis methods in two groups. Section 2.2.1 describes analysis methods applied to the models of programs. The purpose of this section is to give readers a brief background on analysis methods for models, which helps one to understand the necessity for analysis methods on implementation. Section 2.2.2 describes analysis methods for implementations. In section 2.2.2 we describe the goals of these methods and the approaches these methods have taken.

\subsubsection{Analysis on the Model of Systems}

The program development process begins with the model of a program and desired properties for the program. Formal methods are mathematical techniques that describe the models of programs and desired properties. In addition, formal methods can be used to verify the correctness of a program in terms of given properties. A method is formal if it has a sound mathematical basis, typically given by a formal specification language.

An advantage of formal methods is that formal methods reveal the ambiguity, incompleteness, and inconsistency in the model of a program. When formal methods are used in the early program development process, they can reveal design flaws that might otherwise be discovered only during costly testing and debugging phases. During the past two 
decades, tools dedicated to formal methods have been introduced. There have been an increasing number of successful industry case studies using formal methods $[46,58]$.

A weak point of this approach, however, is that the correctness of a model does not necessarily mean that the implementation is correct. This is because the implementation has more detail than the model and is susceptible to errors not present in the model. In addition, there are negative aspects of formal methods including hard to learn/use notations $[33,58]$, high computing power requirements, and primitive tools inadequate for practical usage [58]. Two common formal paradigms are theorem proving and model checking.

\section{Theorem Proving}

The goal of theorem proving is to prove whether a model satisfies given properties. The approach of theorem proving is to prove $M \vdash p$ by mathematical deduction where $M$ is a model of a program given as characteristic statements on the program and $p$ is a property.

An advantage of theorem proving over model checking is that theorem proving provides better scalability to handle infinite state model by induction. Another strong point is that theorem proving uses a more expressive language which includes quantifiers, leading to succinct specification of parameterized systems [15]. The last strong point is that theorem proving works on the syntactical reasoning which provides a relatively complete proof system and can prove any temporal property that indeed holds over the given system.

A weak point of theorem proving is that theorem proving is undecidable as many logic systems are, so that it is hard to be automatic. Due to this limitation, completely automated theorem provers are infeasible. Therefore, theorem proving requires user ingenuity and interaction. Another weak point is that theorem provers suffer from the inability to find counter examples. If the property fails to hold, deductive methods normally do not give much useful feedback, and the user must try to determine whether the fault lies with the system and property being verified or with the failed proof. Finally, theorem proving needs the expert knowledge about the theory used during the proving, which means it is hard to be mastered by the people not working in this area. For a list of theorem provers see [3]. 


\section{Model Checking}

The goal of model checking is similar to that of theorem proving - to prove whether a model satisfies given properties. However, the approach of model checking is different from that of theorem proving. A model checker provides a design specification language for describing a model of a system, which is similar to a programming language but simplified. Also, a model checker provides a property specification language for describing desired properties. A property specification language may be the same as the design specification language. A model checker generates and explores all possible/reachable states of the model and checks whether all the states satisfy the given properties.

An advantage of model checking compared to theorem proving is that describing a model in a design specification language is more familiar to a programmer than describing a model as characteristic statements. Another advantage is that model checking provides automatic verification. In other words, given a model and properties written in formal specification language, the verification process does not require user interaction. Furthermore, model checking can give counter examples which are useful for debugging.

A disadvantage of model checking is that tractable reachability testing algorithms exist for only very simple systems $[60,122]$. In many cases, even those simple systems require large amount of computational resources. (There has been active research on state reduction techniques [121], such as symbolic model checking [113, 138], binary decision diagram reduction [35], and partial order reduction [165]).

To avoid state explosion problem [202], modeling languages have restrictions on the language construct, such as data type and expression [23-25]. Consequently, restriction in the language requires approximation and make modeling of systems difficult. There has been active research on the state reduction technique $[45,121]$, such as symbolic model checking [113, 138], binary decision diagram reduction [35], partial order reduction [165]. Symbolic representations are mainly used for the hardware verification, while partial order reduction is good at the loosely coupled systems. However, symbolic representation is very sensitive to the order of variables. For a list of model checkers, see [3]. 
Symbolic Representation Explicit representation of system models bring tons of states that is hard to be handled by the computer. McMillan [138] proposed a symbolic representation of the states in his dissertation. The basic idea is shown in the following. Any finite-state system can be encoded using a set $\left\{b_{1}, \ldots, b_{n}\right\}$ of binary variables, just as ordinary data types of programming languages are represented in binary form on a digital computer. For this reason, such representations are often called symbolic model checking techniques $[113,138]$. The data structure for it is using Binary decision diagrams $[35,36]$ (BDD)that has following features:

- Every boolean function has a unique, canonical BDD representation.

- Boolean operations can be implemented with complexity proportional to the product of the inputs.

Partial Order Reduction Whereas symbolic model checking derives its power from efficient data structures for the representation and manipulation of large sets of sufficiently regular structure, algorithms based on explicit state enumeration can be improved if only a fraction of the reachable pairs need to be explored. This idea can be successfully applied in the case of asynchronous systems that are composed of concurrent processes with relatively little interaction.

The full transition system has as its runs all possible interleavings of the actions of the individual processes. For many properties, however, the relative order of concurrent actions is irrelevant, and it suffices to consider only a few sequentializations. Reduction techniques that take advantage of the commutativity of actions are therefore often called partial-order reductions. The main problem in the design of a practical algorithm is to detect when two actions commute, given only the "local" knowledge available at a given system state.

The general idea of the partial-order reduction algorithms $[105,166,194]$ is to approximate the semantic notion of commutativity of actions using syntactic criteria. There is always a tradeoff between the potential effectiveness of a reduction method and the overhead involved in computing a sufficient set of actions that must be explored at a given state. 
Moreover, the effectiveness of partial-order reductions in general depends on the structure of the system: it is well suitable for loosely coupled, asynchronous systems instead of tightly synchronized systems.

Unfoldings $[67,68,139]$ are a partial-order approach to the automatic verification of concurrent and distributed systems, in which partial-order semantics is used to generate a compact representation of the state space. For systems exhibiting a high degree of concurrency, this representation can be exponentially more succinct than the explicit enumeration of all states or the symbolic representation in terms of a BDD, thus providing a very good solution to the state-explosion problem.

\section{Software Testing}

Full-scale formalization of complex systems is an extremely expensive activity both technically demanding and labor intensive. In particular, formal proof that an implementation conforms to its specification is rarely cost-effective (in terms of risk reduction). A balance between proof and testing offers a more pragmatic approach. Software testing consists of the dynamic verification of the behavior of a program on a finite set of test cases, suitably selected from the usually infinite executions domain, against the specified expected behavior. Because testing requires the execution of the software, it is often called dynamic analysis. By using formal methods and testing together, we can: Reduce the cost of development by applying testing techniques much earlier in the lifecycle while defects are relatively inexpensive to correct. In the work of [80], the author demonstrated in the form of the Vmodel, the limited benefits that can be accrued when the only formal model available is the code itself. Static analysis of the code can help in deriving adequacy criteria, and dynamic analysis of the code to access whether the criteria have been met. Besides, the author pointed out that the far greater possibilities that exist when formality is introduced into higher layers - the specification and design.

As a form of verification, testing has several advantages over static-analysis techniques (e.g. model checking). One advantage of testing is the relative ease with which many of 
the testing activities can be performed. A second advantage of testing is that the software being developed can be executed in its expected environment. A third advantage of testing is that much of the process can be automated.

Although, as a form of verification, testing has a number of advantages, it also has a number of limitations. Testing cannot show the absence of faults - it can show only their presence. Additionally, testing cannot show that the software has certain qualities. Moreover, test execution results for specific test cases cannot usually be generalized. Software testing is mostly about empirical correctness, and historically testing techniques have been applied, quite naturally, at the code level. Testing by its nature can never conclude anything mathematically valid, as it amounts to taking a sample and trying to infer a generally valid judgment on the whole from the observed part.

\section{Runtime Verification}

Runtime verification is considered as a so-called light-weighted formal analysis method. Runtime verification is a method or algorithm to analyze the execution trace of a running program based on a formal specification and concludes a certain property about that program. Two main features are exposed in the runtime verification - a formal specification of system property and the execution trace to be analyzed. A runtime checker takes a system and properties as input, check the system against properties when the system is running.

Currently, most of runtime verification tool use temporal logic(TL) as the specification language of the program. For instance, Temporal Rover and DBRover tools [8] translates LTL properties into comments, which are inserted at chosen positions in the program. $\mathrm{JPaX}[95]$ presented three algorithms with some optimization to monitor future and past linear time temporal logic (LTL). Java MultiPathExplorer [184] is a tool that checks a past time LTL safety property against a partial order extracted online from an execution trace. Java-MoP [39] is a generic logic monitoring tool for a paradigm merging specification and implementation. POTA [183] is a partial order trace analyzer system that analyzes distributed execution traces. Almost all of these tools are working on the code level. Next, 
we enumerate design issues on the runtime formal analysis and the rationale for design decisions. These issues are general issues, not specific to certain system architecture.

Infrastructure Runtime verification, a light-weighted formal analysis, is more practically feasible than so-called heavy-weighted formal analysis on the large complex systems, in terms of scalability and on-line checking. Scalability is a feature of a technique that should be practically applicable to large systems consisting of thousands or millions of lines of code. Because the behavior of a system in the execution might be different from that in the static, e.g. data racing and deadlock among threads, checking system online is quite necessary.

Currently the runtime verification monitor or observer is generally implemented in the following processes. The system is designed to have two basic functions. First function is the ability to discharge the program execution events as soon as possible, in order to minimize the space requirements. Second is the ability to check the observable events against the system specification, in most cases are formally specified. The typical example is $\mathrm{JPaX}$ [95], which is composed of two modules - instrumentation and observer. Instrumentation module is responsible for extracting event traces and feed them to the observer, while observer is to check high-level and/or low-level properties. JavaMaC $[119,126]$ consists of three parts (filter, event recognizer, and runtime checker), however, the filter and event recognizer are the part that deals with the event extraction and feeding. Although some method, e.g. AspectJ, can weave the instrumentation by join point and advice, the basic functionality of this part cannot be weaved. The systems that using insertion, e.g. Temporal Rover and DBRover [8], do not have a clear two-module infrastructure. However, the insertion procedure and checking code are corresponding to these two modules.

This two-module infrastructure does not depend on any logic and programming language. One of the most obvious benefits of this infrastructure is that the observer/monitor can be executed in parallel with the monitored program and can be implemented distributed. 
Program Execution Trace and Its LTL Semantics The events can be categorized as low-level and high-level events. In the case of runtime verification of the system model in the implementation, we have both kinds of events. The low-level events are variables, method calls, thread switch, etc., and the high-level events are those are relevant to the specification elements. In the case of monitoring system model in the implementation level, low-level events are actually those model elements that are mapped into the implementation level.

The interpretation of the execution traces depend on the logic we used or the specification language of the system. In the standard temporal logic, the event trace is infinite while in the practice we use finite trace with the last state stuttering. The linear temporal logic semantics in the event traces can be recursively defined using rewriting logics. We present the semantics of execution trace semantics in terms of propositional LTL as follows [88, 92, 93, 95, 177]:

Assume two total functions on traces, head: Trace $\rightarrow$ Event returning the head event of a trace and length returning the length of a finite trace, and a partial function tail : Trace $\rightarrow$ Trace for taking the tail of a trace. That is, head $(e, t)=\operatorname{head}(e)=e, \operatorname{tail}(e, t)=t$, and length $(e)=1$ and length $(e, t)=1+$ length $(t)$. Assume further for any trace $t$, that $t_{i}$ denotes the suffix trace that starts at position $i$, with positions starting at 1 . The satisfaction relation $\vDash$ Trace $\subseteq$ Formula defines when a trace $t$ satisfies a formula $f$, written $t \vDash f$, and is defined inductively over the structure of the formulae as follows, where $A P$ is any atomic proposition and $\phi_{1}$ and $\phi_{2}$ are any formulae 1 :

In our work we use this event trace semantics.

Formula Transforming The formal specification logic cannot be directly used as the observers. The logic formulae are first transformed into finite state automatons and then can be used for the input file of the runtime checker. Currently, there are two main methods in the transformation of linear temporal logic. One method is called automata-based and another is rewriting-based. 
Table 1: Event trace semantics of TL formula

$$
\begin{aligned}
& t \vDash t r u e \quad \text { iff } t \text { is always true } \\
& t \vDash \text { false } \quad \text { iff } t \text { is always false } \\
& t \vDash p \quad \text { iff } t \neq \epsilon \text { and head }(t) \text { is } p \\
& t \vDash \phi_{1} \text { op } \phi_{2} \quad \text { iff } t \vDash \phi_{1} \text { and/or/xor/implies/iff } t \vDash \phi_{2} \\
& \text { when } o p \text { is } \vee / \wedge / \oplus / \rightarrow / \leftrightarrow \\
& t \vDash \bigcirc \phi \quad \text { iff } t \neq \epsilon \text { and } \operatorname{tail}(t) \vDash \phi \\
& t \vDash \diamond \phi \quad \text { iff } \exists 1 \leq i \leq \text { length }(t)+1: t_{i} \vDash \phi \\
& t \vDash \square \phi \quad \text { iff } \forall 1 \leq i \leq \text { length }(t): t_{i} \vDash \phi \\
& t \vDash \phi_{1} U \phi_{2} \quad \text { iff } \exists 1 \leq i \leq \text { length }(t)+1: t_{i} \vDash \phi_{2} \text { and } \\
& \forall 1 \leq j \leq i: t_{j} \vDash \phi_{1} \\
& t \vDash \diamond \phi \quad \text { iff } \exists 1 \leq i \leq \operatorname{length}(t): t_{i} \vDash \phi \\
& t \vDash \square \phi \quad \text { iff } \forall 1 \leq i \leq \text { length }(t): t_{i} \vDash \phi \\
& t \vDash \phi_{1} \mathcal{S} \phi_{2} \quad \text { iff } \exists 1 \leq i \leq \text { length }(t): t_{i} \vDash \phi_{2} \text { and } \\
& \forall 1 \leq j \leq i: t_{j} \vDash \phi_{1}
\end{aligned}
$$

\section{Automata-based Transformation.}

In the automata-based method, there are two groups. Bernd Finkbeiner etc. [69] using alternating automata [196] to represent the temporal logic formula. They proposed three basic algorithms based on the transformation and some optimization on these algorithms. Depth-first search is easy to be implemented by inefficient in that it may traverse part of a trace multi-times. The breadth-first algorithm traverses the trace once but it may have an exponential number of sets of nodes. Backward traversal can avoid these issues and it was also proposed and discussed in the work [92, 177]. The advantage of using alternating automata to represent the language accepted by temporal formula is that the alternating automaton that accepts the same language as the formula is linear in the size of the formula, while it is worst-case exponential for nondeterministic or $\forall$-automata.

In the work of [82], the authors proposed a method that transform LTL formula into Büchi automata, which is efficiently implemented in the TaX (Trace analyZer) that is integrated in the JPaX. The algorithm presented in the work [82] is based on an efficient tableau-like LTL to Büchi automata translation. The difference of this work 
from the work in [69] is that this work uses a deterministic automaton. But nondeterministic automata are more concise than deterministic one.

The event trace semantics of LTL is infinite in the sense of automata, which is different from the above semantics.

2. Rewriting-based Transformation.

Rewriting logic [144] is logic of concurrent change that can naturally deal with state and with highly nondeterministic concurrent computations. It has good properties as a flexible and general semantic framework for giving semantics to a wide range of languages and models of concurrency. Membership logic [32] is a sublogic of rewriting logic. Maude [47] is a high-level meta-language that support both equational and rewriting logic computation. JPaX [95] is an example of the rewriting-based transformation which uses Maude [47] as logic engine. In the paper [95], they presented 8 rules to translate LTL based on several functional modules which are theories in membership equational logic [32]. Rewriting logic semantics was introduced before.

Translation Mechanisms From Model to Program Language The translation mechanism or a mapping methodology from the system design model to the programming language is necessary and critical for the runtime verification application on the system model. It aims at systematically constructing a relationship between high level model of the system and its mirrored low level implementation. The benefits of the translation are listed as follows:

- allow the model checking in the programming language context.

Model is a high level abstraction of the system and often written in the mathematical expressions to be concise and precise. These expressions cannot be recognized by the programming language compiler without the effort on the translation. In this case, the target language is the programming langauge that is mapped from the model. 
- bridge the gap between the high level specification and its implementation.

Model is an abstract description of software systems thus many issues are ignored in order to obtain the high-level structure and behavior in the design. Many system implementations are hard to follow the model description because of the environment of the system implemented. There is a gap between system models and their mirrored implementation. Without a systematic translation, the model does not make sense for the released systems.

Program Instrumentation Program instrumentation is a procedure for inserting monitoring code into monitored program to get dynamic behaviors of monitored program. It can be done in both source code and byte code level to extract relevant events. Many program instrumentation tools are designed on different programming languages, such as $\mathrm{C}$, $\mathrm{C}++[159]$ and Java [18] code in the byte code level.

Valgrind [159] implements bytecode instrument in two ways, one is using insertions to put calls to $\mathrm{C}$ functions, another is extending the UCode with new instructions. The BCEL (Byte Code Engineering Library) [50] is a toolkit for the static analysis and dynamic creation or transformation of Java class files. It enables developers to implement the desired features on a high level of abstraction without handling all the internal details of the Java class file format and thus re-inventing the wheel every time. BIT (Bytecode Instrumenting Tool) [125] is a collection of Java classes that allow one to build customized tools to instrument Java Virtual Machine (JVM) bytecodes. jContractor [18] performs on-the-fly bytecode instrumentation to detect violation of the contract specification during a program's execution. jContractor is a pure Java library providing a rich set of syntactic constructs for expressing contracts and it allows the checking without source code. jMonitor [114] is a pure Java library and runtime utility for specifying event patterns and associating them with user provided event monitors. jMonitor instrumentation works at the Java bytecode level and does not require the presence of source code for the Java application monitored. The class bytecodes of the monitored Java program are instrumented on the fly by the jMonitor 
class loader according to the needs of the externally specified jMonitor event patterns and event monitors.

Jass [27], Java with assertions, allows annotating Java programs with specifications in the form of assertions. The Jass tool translates annotated assertions into Java programs in which compliance with the specification is dynamically tested. Jass supports two groups of annotations, classical program verification (e.g. pre- and postconditions, invariants) and behavioral subtyping for the trace assertions using JDI (Java Debugger Interface). Jass is a pre-compiler which is written in Java.

Compared with manual assertion, some tools can automatic instrument programs. ANNA (ANNotated Ada) $[181,192]$ is a generalized assertion checking system for annotated Ada programs. Given Ada program, a user makes annotations written in ANNA language. ANNA provides classes of annotations for monitoring different entities-object, subtype, statement, subprogram, result and exception. Given annotated Ada program, ANNA compiler compiles annotations into corresponding inline Ada codes or checking functions or tasks depending on the annotations. Then, ANNA compiler puts these code into proper place of the source code. ALAMO (A Lightweight Architecture for MOnitoring) $[110,111,193]$ is a monitoring system for ANSI C programs. ALAMO monitors every activity in syntax level, such as addition, assignment, loop. The configuration language provides which activities are to be monitored. Based on the configuration, given ANSI C source code, ALAMO instruments the source code following the configuration.

There is an interesting relation between program instrumentation and aspect-oriented programming [118]. Aspect-oriented is one of software development methodology that complements object-oriented method in the system design and development process. It performs cross-cutting on different concerns of system subunits or components. In comparison, the runtime verification is put forth something done more after the fact. Runtime verification is to deal with some aspects in different units of a running application that concerns properties specified. These properties are related with different classes, functions and can be observed on the interleaving execution with the running application. Furthermore, 
some work using Aspect $\mathrm{C}++[134]$ on the instrumentation of $\mathrm{C}++$ code proved that little performance overhead is introduced into the monitored program, which is better than that of $\mathrm{C}++$ instrumentation. Runtime verification workshop 2007 [179] is to create a synergy between the fields of runtime verification and aspect oriented programming.

\section{Summary}

Software Quality Assurance (SQA) aims to ensure that the final product will have an acceptable quality. It is mainly a management activity to identify quality problems early in the development. In the quality engineering, to accurately assure the correctness of software systems, formal methods is necessarily applied. The above discussion about the formal analysis of software design can be summarized in the Figure 2.

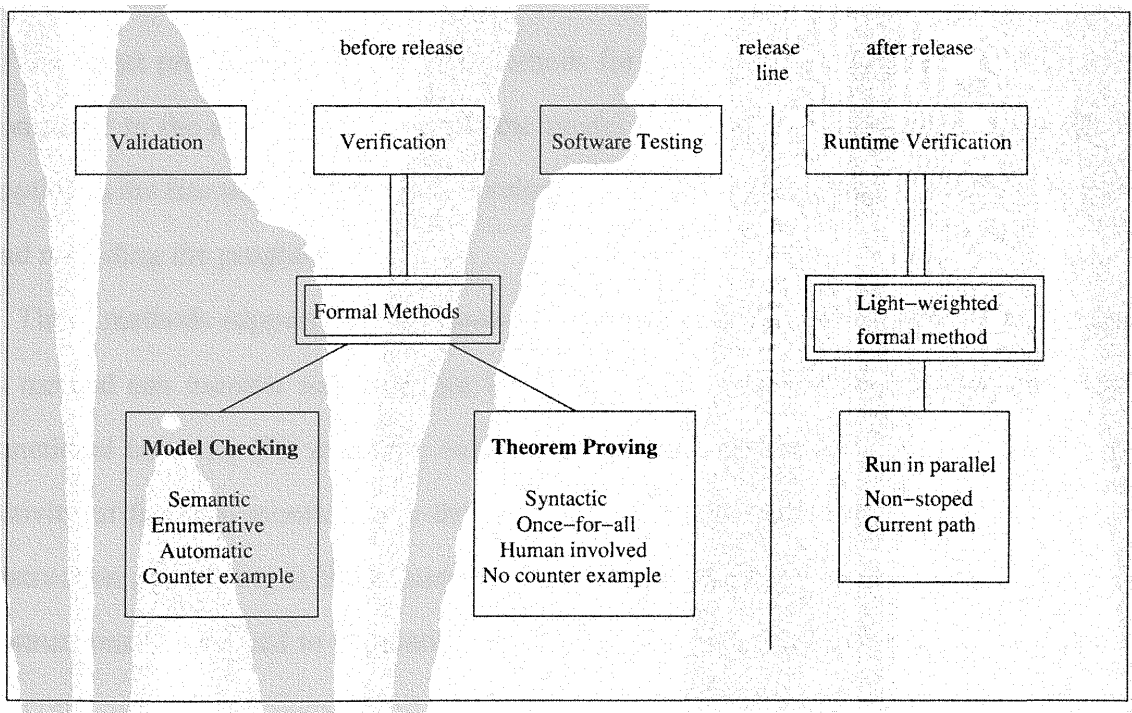

Figure 2: Summarization of the formal analysis methods of the software design

\subsubsection{Analysis of Implementation}

Methods for the implementation of a program check whether executions of the program satisfy given properties. These methods are applied in later stages of the program development process because they require the implementation of the program. 
An advantage of these methods is that they can check actual executions of a program. Although a model is proven correct, however, it does not necessarily indicate that the implementation is correct; the implementation has more detail than the model and is susceptible to errors not present in the model. For example, a model may implicitly assume a natural number can be arbitrarily large. However, an implementation may restrict a natural number to be less than $2^{32}$ because the implementation chooses to represent a natural number in 32 bits. Then, overflow error which may happen in the implementation cannot be detected in the model. Furthermore, there is no guarantee that implementation is strictly following a model because there can be errors caused by human programmers who implement the program after understanding the model.

These methods, however, do not provide mathematical guarantees on the correctness of the target program as the analysis methods for the model do. Another negative when compared to the analysis methods for the model of a program is that these methods are applied in the late stages of program development process, which leads to costly debugging and re-coding the program.

These methods support different levels of monitoring according to application purpose. A method can monitor unit behavior (for example, communication between processes), functional behavior (for example, step-by-step instruction trace). The finest-level target activity of function monitoring is the execution of a statement in a source code. That of instruction monitoring is the execution of an instruction. A statement consists of several instructions. Compared to instruction monitoring, function monitoring needs to recognize all consecutive instructions forming the statement. We classify analysis methods on implementation into the following three groups according to the level of monitoring.

1. unit monitoring: to monitor behaviors intra-processes;

2. function monitoring: to monitor behaviors inter-processes;

3. instruction monitoring: to monitor behaviors in an instruction level, more specific into the machine level. 
The level of monitoring affects the amount of overhead and the instrumentation stages in general. For example, analysis methods for process-level behavior instrument the communication interface between processes. Analysis methods for instruction-level activity instrument the run-time execution environment on which each instruction of the target program is executed. Details of three levels of monitoring are discussed in the follows.

\section{Unit Monitoring}

The goal of this group of methods is to monitor externally observable behavior of processes such as input/output or communication between processes. An advantage of these methods is that they decrease the difficulty of instrumenting target program by instrumenting only an interface of a process that is well defined and observable outside the process. Another benefit is its low overhead cost. Each process is considered as a unit so the interaction of it with the outside is the only concern for the monitoring. The external behavior of a process generates less frequent events compared to the internal behavior. This leads to less overhead in the instrumented target program. A drawback to these methods is that they check only external behavior of processes. They cannot check desirable properties concerning internal behavior of a process. For example, suppose a process has a stack implementing push() and pop(). A non-emptiness property stating that the number of pop()'s should be less than the number of push()'s cannot be checked in process-level monitoring. In other words, unit monitoring cannot detect a violation in internal behavior which does not effect external behavior or at least not until the violation corrupts external behavior eventually. Even when an error in external behavior is detected, the source of the error inside of a process is hard to find. Some systems performing unit monitoring are described below.

Model-based testing aims to detect violations by using a formal model of the program. Testing oracle takes input/output execution of a program and check whether the execution is correct with regard to the formal model of the program. The difference between these works is mainly the formalism they use to describe a model. Supervisor [182] compares 
the output of a target program and expected output generated by interpreting the model of the program written in SDL $[11,12]$. Dillon [53] develops an algorithm to generate a testing oracle from a Graphical Interval Logic specification [52]. Clarke [43] conducts a case study on automatic testing of Philips Audio Control Protocol using a specification written in ACSR [34].

JEM (Java Event Monitor) [129] is an event-mediator type of system like the CORBA event channel. In other words, JEM provides its own communication channel similar to the CORBA event channel. A benefit of using its own event channel rather than a standard event channel is freedom in defining primitive events. However, using its own event channel does not scale well compared to using a standard event channel.

\section{Function Monitoring}

The goal of this group of methods is to monitor internal behavior of processes at a statement-level. For example, a non-emptiness property that the number of pop()'s should be less than the number of push()'s in a stack can be monitored at this level of monitoring. A strong point of these methods is that these methods can check various desirable properties not limited to properties on external behavior. A weak point of these methods is that the instrumentation of a target program for monitoring various activities is a complicated task. Deciding where to insert probes into a program in general is a nontrivial task [188] because it requires knowledge of program structure. Furthermore, instrumentation may modify the semantics of the target program if thorough care is not taken. For example, suppose a return statement is inserted into a method accidently. The statements of the method after the return would not be executed. This is a serious problem especially for manual instrumentation. Second, source code is usually only available to the developers of a program. In many cases, though not all cases, these methods require the source code of a program in order to recognize and instrument target statements. The prototype tool - SAM Parser, belongs to this category. 
ALAMO (A Lightweight Architecture for MOnitoring) [110,111] is to reduce the difficulties in writing monitoring tools by constructing a platform on which monitor construction is relatively easy. However, accessibility to the entire set of states in the target program causes 2-3 orders of magnitude overhead. Since SAM Parser uses online instrumentation, there is very light overhead. $\mathrm{MaC} \mathrm{MaC} \mathrm{[126]} \mathrm{architecture} \mathrm{monitors} \mathrm{events} \mathrm{generated} \mathrm{from}$ three types of statements: variable assignments, method invocation, and method returns. Thus, the $\mathrm{MaC}$ architecture does not cause such high overhead. A monitor and a checker of the $\mathrm{MaC}$ architecture are generated automatically from the requirement specifications. The $\mathrm{MaC}$ architecture provides a low-level specification language to define primitive events In addition, the $\mathrm{MaC}$ architecture instruments the target program automatically according to the definitions of primitive events. SAM Parser architecture is similar as MaC's. We will compare them in Chapter 6. JRTM (Java Run-time Timing constraint Monitor) [150,151] aims to detect violation of timing properties using Real-Time Logic (RTL) [109] as a property specification language. Based on informal definitions of primitive events, the target program is instrumented manually. Unless instrumentation is performed correctly, the analysis cannot produce the correct result. SAM Parser instruments program automatically which ease lots of work and reduce errors in the most extent. Sentry System [41,42] aims at a low-cost, low-precision monitor. Sentry is a monitor watching over the behavior of a target program continuously. Time Rover [8] is to detect the violations of assertions over target program variables. Assertions are written in temporal logic at run-time. Time Rover uses a normal temporal logic and metric temporal logic which extends the temporal logic by supporting the specification of real-time constraints to temporal operators. Also, the temporal logic used by Time Rover supports counting operators so that a user can specify the number of event occurrences in the assertion. A user has to write and insert assertions into a target source code. Time Rover supports various languages such as Java, $\mathrm{C}++$, and Verilog. 


\section{Instruction Monitoring}

The purposes of this group of analysis methods are 1) to monitor and check the behavior of the target program in terms of instruction-level activities; 2) is to steer the target program. These methods provide a virtual machine as run-time execution environment to monitor the execution of the target program instructions one by one. An advantage of these methods is that they do not require the instrumentation of a program because a virtual machine can monitor execution of a target program as it is without instrumentation. This removes the difficulty of instrumenting a target program. In addition, a code size does not increase due to inserted probes. Furthermore, these methods can monitor and check the behavior of the target program at a finer-grained level, compared to the function monitoring methods. Finally, these methods provide the accessibility and controllability of entire set of target program states. A weak point is that this fine-grain monitoring capability costs a run-time overhead magnitude of 2-3 because of the slow interpretation speed of a virtual machine. In addition, these methods are machine dependent. Furthermore, these methods need a specialized run-time execution environment rather than the existing environment which is reliable and familiar to users. This requirement may prohibit these methods from being widely applied. Finally, reasoning about high-level properties based on instruction-level behavior is not an easy task.

Dynascope [189-191] serves as an instruction-level behavior monitor such as an array bound checker. A weak point is that Dynascope does not provide a requirement specification language. As an event-based debugger for $\mathrm{C}$ programs, Dalek $[160,161]$ is to detect composite events from the instruction level execution of target program. JPDA (Java Platform Debugger Architecture) [4] provides a debugging interface so that a programmer can access information available on JVM and manipulate the execution of the target program.

We summarize the above discussion based on the features to be examined for the monitored program in the Table 2 . 
Table 2: Summary of Implementation Analysis

\begin{tabular}{|c|c|c|c|c|c|c|c|}
\hline \multicolumn{2}{|c|}{ Methods } & $\begin{array}{l}\text { High Level } \\
\text { Specification }\end{array}$ & $\begin{array}{l}\text { Code } \\
\text { Size }\end{array}$ & $\begin{array}{l}\text { Overhead } \\
\text { Overhead }\end{array}$ & $\begin{array}{l}\text { Event } \\
\text { Frequency }\end{array}$ & $\begin{array}{l}\text { Instru. } \\
\text { Complex. }\end{array}$ & $\begin{array}{l}\text { Property } \\
\text { Class }\end{array}$ \\
\hline \multirow{2}{*}{$\begin{array}{l}\text { Unit } \\
\text { Monitoring }\end{array}$} & Model-based & Yes & Small & Small & Low & Low & No \\
\hline & JEM & Yes & No & Small & Low & No & No \\
\hline \multirow{6}{*}{$\begin{array}{l}\text { Function } \\
\text { Monitoring }\end{array}$} & ALAMO & No & Large & Large & Medium & Auto & No \\
\hline & MAC & Yes & Medium & Medium & Medium & Auto & No \\
\hline & JRTM & Yes & Medium & Medium & Medium & High & No \\
\hline & Sentry & Yes & Medium & Small & Medium & Auto & No \\
\hline & Time R. & Yes & Medium & Medium & Medium & High & No \\
\hline & SAM Parser & Yes & Medium & Low & Low & Low & Yes \\
\hline \multirow{3}{*}{$\begin{array}{l}\text { Instruction } \\
\text { Monitoring }\end{array}$} & Dynascope & No & No & Low & High & No & No \\
\hline & Dalek & No & No & Low & High & No & No \\
\hline & JPDA & No & No & Low & High & No & No \\
\hline
\end{tabular}

\subsection{Translation Correctness}

Translation is a necessary unavoidable step in most cases for the verification of the system model at both design and implementation program. In the verification of design level, the tools require certain programming language that may not be the specification of the model. It is necessary to translate from the model to the program languages recognized by model checkers. In the verification of implementation level, the specification language of the model may not be same as language for the program, then similar situation happens. In both situations, a validation process is necessary to show the correctness of the translated code being consistent with the original model. This procedure is similar as the procedure defined in the "verified compiler" [102] - the output from a compiler is consistent with the input program, which is proposed by C. R. Hoare in the "Grand Challenge" in 2003. Currently, several research works have been done in the translation validation.

Pnueli [173] etc. presented the notion of translation validation as a new approach to the verification of translators (compilers, code generators). In their approach each individual translation is followed by a validation phase which verifies that the target code produced on this run correctly implements the submitted source code. [172] presented an automatic code validation tool based on the uninterpreted functions and symbolic represented models.

There are many works have been done on the translation from the formal specification to a model checker programming languages. The basic goal of translation from the formal specification to the model checker programming language is to verify the system properties 
specified in the specification. [78] presented a set of rules that translate from Petri nets to Promela for use within SPIN. [99] presented two translations, one is from high level Petri nets to SMV, another one is from high level Petri nets to FTS (Finite Transition System). Both translations are necessary for the system properties verification and analysis. The soundness of the second translation was also justified. [98] discussed the analysis method on the SAM [198] architecture model using SMV. In this work, several translation rules from SAM to SMV are provided. These rules are consistent with the stepwise translation algorithm in the work [99]. WRIGHT [21] and DARWIN [133] are two other software architectures that have been successfully verified by model checking techniques. In summary, the translation step is necessary for most model checking process. Currently, however, the justification and validation of the translation steps are less provided. 


\section{CHAPTER 3 \\ PRELIMINARIES}

\subsection{Introduction}

As discussed in Chapter 1, the integrated framework is proposed for verification and validation of software architecture model in both design and implementation level.

In this chapter, Section 3.2 gives a brief introduction of Software Architecture Model - SAM, including two formalisms - Petri nets and temporal logic. Two object oriented programming language used in the target code ArchJava and AspectJ will be discussed in Sections 3.4 and 3.5 respectively.

\subsection{SAM - Software Architecture Model}

SAM, an architectural description model based on Petri nets and Temporal logic, is wellsuited for modeling distributed systems. SAM architecture model is hierarchically defined as follows. A set of compositions $C=\left\{C_{1}, C_{2}, \ldots, C_{k}\right\}$ represents different design levels or subsystems. A set of component $C_{m_{i}}$ and connectors $C_{n_{i}}$ are specified within each composition $C_{i}$ as well as a set of composition constraints $C_{s_{i}}$, e.g. $C_{i}=\left\{C_{m_{i}}, C_{n_{i}}, C_{s_{i}}\right\}$. In addition, each component or connector is composed of two elements, a behavioral model and a property specification, e.g. $C_{i j}=\left(S_{i j}, B_{i j}\right)$. Each behavioral model is described by a PrT net, while a property specification by a temporal logical formula. The atomic proposition used in the first order temporal logic formula is the ports of each component or connector. Thus each behavioral model can be connected with its property specification. A component $C_{m_{i}}$ or a connector $C_{n_{i}}$ can be refined to a low level composition $C_{l}$ by a mapping relation $h$, e.g. $h\left(C_{m_{i}}\right)$ or $h\left(C_{m_{i}}\right)=C_{l}$. Fig. 3 shows a graphical view of a simple SAM architecture model.

Fig. 18 shows the SAM model of coffee machine with three components and three connectors. From this figure, we can see the coffee machine itself is modelled as a composition 


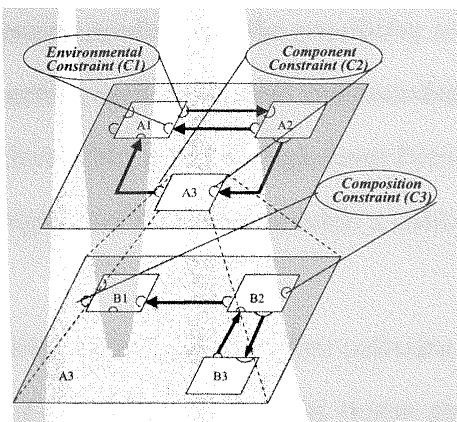

Figure 3: A SAM Architecture Model

CoffeeMachine, which has three sub components: CMInterface, CoinHandler, and BrewingFacility. The example coffee machine is discussed in the Chapter 8 . In this section, I introduce two component methods integrated in SAM model: Petri nets and Temporal Logic.

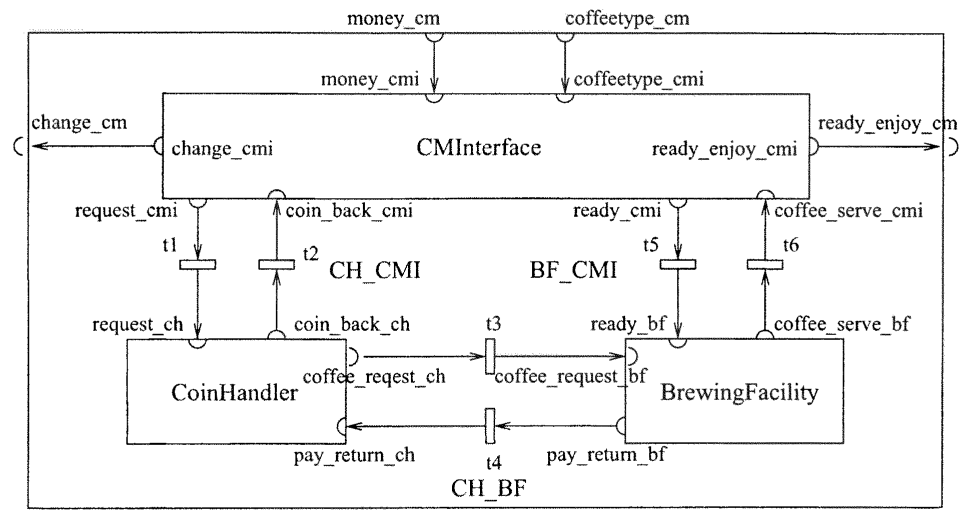

CoffeeMachine

Figure 4: SAM Model of Coffee Machine

\subsubsection{Petri Nets}

Petri nets [153], introduced by Dr. Carl Adam Petri in his PhD thesis (Kommunikation mit Automaten), is a formal and graphical appealing language that is appropriate for modeling concurrent and distributed systems. A main motivation for the use of Petri nets in 
concurrent and distributed systems modeling is the possibility to formally state and decide certain desirable system properties, such as liveness and boundedness. There are in general two kinds of Petri nets: low-level Petri nets and high-level Petri nets. Although they have the same expressive power, high level Petri nets provide a more succinct and manageable system description.

In the development of reliable, secure, and error-free software systems the application of Petri nets is a well-known approach. Petri nets with its syntax and structure are suitable for the description of concurrent and distributed systems. In the following we use PrT nets as an example to introduce the syntax and semantics of high level Petri nets.

\section{Predicate/Transition Nets}

We introduce Predicate Transition Nets (PrT Nets) as an example of a high level Petri net.

Definition 1 (PrT Nets) $A$ Predicate/Transition net is a 8 -tuple $\left(P, T, F, \Sigma, \varphi, L, R, M_{0}\right)$, where:

1. $P$ is a finite set of places, $T$ is a finite set of transitions $(P \cap T=\Phi, P \cup T \neq \Phi)$, and $F$ is a set of arcs or flow relations between each pair of $P$ and $T$, e.g. $F \subseteq(P \times T) \cup(T \times P)$. The tuple (P, T, F) forms a basic Petri net structure.

2. $\Sigma$ consists of some sorts of constants together with some operations and relations.

3. $\varphi$ is a relation associated each place $p$ in $P$ with a subset of sorts.

4. $L$ is a labelling function on arcs. Given an arc $f \in F$, the labelling function of $f$, $L(f)$, is a set of labels, which are tuples of constants (CONs) and variables $(X)$. If $f \notin F, L(f)=\Phi$.

5. $R$ is a mapping from transitions to a set of inscription formulae. The inscription on transition $t \in T, R(t)$, is a logical formula built from variables and the constants, operations, and relations in structure $\Sigma$; variables occurring free in a formula have to occur at an adjacent input arc of the transition. 
6. $M_{0}$ is the initial or current marking with respect to sort, which assigns a multi-set of tokens to each place $p$ in $P$ with the same sort, $M_{0}: P \rightarrow M C O N s$.

Dynamic semantics of PrT nets:

1. A marking $M$ of a PrT net $N$ is a mapping function defined from set of places $P$ to constants MCONs.

2. The enabling condition of a transition $t \in T$ under a marking $M$ with a substitution $\alpha=\left\{x_{i} \leftarrow c_{i} \mid x_{i} \in X, c_{i} \in M C O N s\right\}$ is defined as follows:

$\forall p \in P .(L(p, t): \alpha) \subseteq M(p) \wedge R(t): \alpha$

3. If a transition $t \in T$ under a marking $M$ with a substitution $\alpha$ is enabled, a marking $M^{\prime}$ is obtained after the transition $t$ is fired, then the firing condition of a transition $t$ is defined as:

$\forall p \in P . M^{\prime}(p)=M(p)-L(p, t): \alpha \cup L(t, p): \alpha$.

In PrT nets, each place is assigned a sort indicating what kind of tokens it can contain. The tokens in a place can be viewed as a multi-set over the sort. A marking of a PrT net is a function that assigns tokens to each place. A label is assigned to each arc to describe types and numbers of tokens that flow along this arc. Each transition has a boolean expression called guard, which specifies the relationship among arcs related with the transition. Petri nets in Fig. 21 demonstrates the behavior model of the components in the coffee machine example (in Chapter 8).

For example, in Fig. 21 transition input is enabled in the marking $M_{0}$ under the assignment $f$ such that $f(x)=50, f(y)=2$. After firing of transition input under assignment $f$, $M($ money_cmi $)=M($ coffeetype_cmi $)=\emptyset, M($ request_cmi $)=\langle 50,2\rangle, M($ sig $)=$ true, and the tokens in other places remain the same.

\subsubsection{Temporal Logic}

Temporal logic defines each propositional predicate calculus on a time slot. It is specifically referred to the modal-logic introduced around 1960 by Arthur Prior [174] under the 


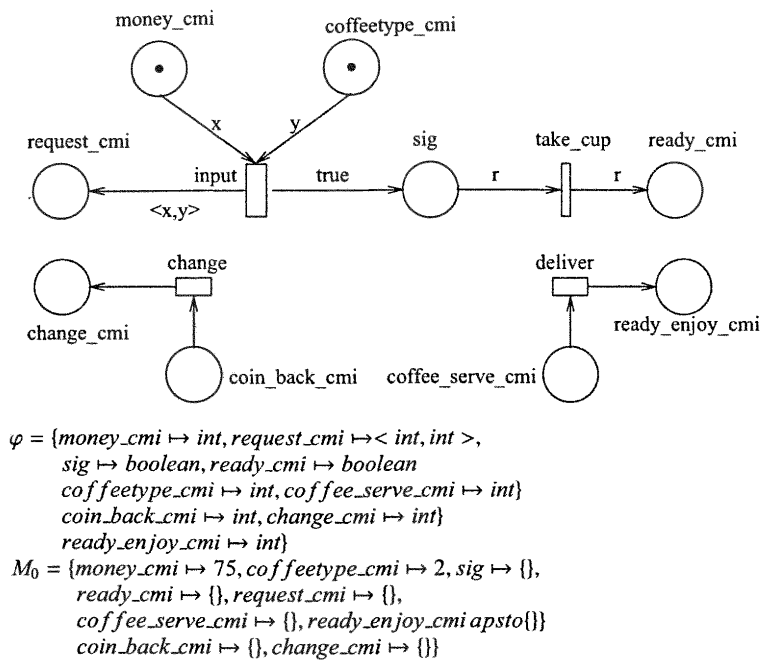

Figure 5: Behavior of a Subcomponent (CMInterface) in CoffeeMachine

name of Tense Logic and subsequently developed further by logicians and computer scientists. In 1977, Amir Pnueli [171] extended the tense logic with four operators $(\square, \diamond, \bigcirc$, $\mathcal{U})$ for future time linear temporal logic and four for past time ( $\square$, Diamonddot, odot, $\S)$ and concerned with the specification and verification of programs, especially concurrent programs in which the computation is performed by two or more processors working in parallel.

Temporal logic [136] is a widely used logic for specifying properties of reactive and concurrent systems. Formulae in classical Linear Temporal Logic (LTL) can be built using the following operators:

$$
\begin{aligned}
F::= & \text { true } \mid \text { false }|A P| \neg \phi \mid \phi_{1} \text { op } \phi_{2} & & \text { Propositional operators } \\
& \circ \phi|\diamond \phi| \square \phi \mid \phi_{1} U \phi_{2} & & \text { Future time operators } \\
& \odot \phi|\diamond \phi| \square \phi \mid \phi_{1} S \phi_{2} & & \text { Past time operators }
\end{aligned}
$$

The semantics of temporal logic is defined on the system behaviors (infinite sequences of states). The behaviors are obtained from the execution sequences of petri nets where the last marking of a finite execution sequence is repeated infinitely many times at the end 
of execution sequence. For example, for an execution sequence $M_{0}, \ldots, M_{n}$, the following behavior $\sigma=\ll M_{0}, \ldots, M_{n}, M_{n}, \ldots \gg$ is obtained, where $M_{i}$ is a marking of the petri net.

Let $\sigma=\ll M_{0}, M_{1}, \ldots \gg$ be the behavior, where each state $M_{i}$ provides an interpretation for the variables mentioned in predicates. Let $D$ be the data domain. The semantics of a temporal formula $p$ in behavior $\sigma$ and position $j$ is denoted by $(\sigma, j) \vDash p$. We define:

- For a state formula $p,(\sigma, j) \vDash p \Leftrightarrow M_{j} \vDash p$;

- $(\sigma, j) \vDash \neg p \Leftrightarrow(\sigma, j) \forall p ;$

- $(\sigma, j) \vDash p \vee q \Leftrightarrow(\sigma, j) \vDash p$ or $(\sigma, j) \vDash q$;

- $(\sigma, j) \vDash \square p \Leftrightarrow(\sigma, i) \vDash p$ for all $i \geq j$;

- $(\sigma, j) \vDash \diamond p \Leftrightarrow(\sigma, i) \vDash p$ for some $i \geq j$;

- $(\sigma, j) \vDash p U q \Leftrightarrow \exists i \geq j:(\sigma, i) \vDash q$, and $\forall j \leq k<i,(\sigma, k) \vDash p$;

- $(\sigma, j) \vDash \forall x \in D . p(x) \Leftrightarrow$ for any element $m$ in $D,(\sigma, j) \vDash p(m)$;

- $(\sigma, j) \vDash \exists x \in D . p(x) \Leftrightarrow$ for some element $m$ in $D,(\sigma, j) \vDash p(m)$;

The models of LTL are infinite execution traces, reflecting the behavior of such systems as ideally always being ready to respond to requests, operating system being typical example. LTL has been mainly used to specify properties of finite-state reactive and concurrent systems, so that the full correctness of the system can be verified automatically. In the real environment, the event sequence is finite instead of infinite. There are two possible options currently to describe this finite trace using that infinite semantics. We think the most appropriate assumption to make at the end of the trace is that it is stationary in the last state. Then its semantics can be defined as the finite trace is infinitely extended by repeating the last state. Another option would be to consider that all atomic predicates are false or true in the state following the last one, but this would be problematic when inter-dependent predicates are involved, such as "gate-up" and "gate-down". The semantics of finite trace LTL is described in the next chapter. 
Property specifications for each component/connector in SAM are defined by LTL formulae. Some heuristic rules of how to specify temporal properties are given in [97]. The following is a property of component CoffeeMachine called Request:

$$
\begin{aligned}
& ((\text { money_cmi }(85) \wedge \text { coffeetype_cmi }(2)) \longrightarrow \\
& \diamond(\text { change_cmi }(85) \vee(\text { change_cmi }(10) \wedge \text { ready_enjoy_cmi }(1))))
\end{aligned}
$$

This formula indicates that (Fig. 21), if the place money_cmi has token 85 and place coffeetype_cmi has token 2 , in the some future time, either we will has token 85 in the place change_cmi and token 1 in the place ready_enjoy_cmi.

\subsection{Maude}

\subsubsection{Introduction}

Maude [48] is a freely distributed high-performance system, supporting both rewriting logic and membership equational logic. Because of its efficient rewriting engine and its metalanguage features, Maude turns out to be an excellent tool to create executable environments for various logics, theorem provers, and even programming languages.

In Core Maude [48] the basic units are functional modules and system modules. Maude's functional modules define data types and operations on them by means of equational theory, which is an extension of ordered equational logic called membership equational logic [146]. A Maude system module specifies a rewrite theory. We explain the rewrite theory and equational theory as follows.

\subsubsection{Rewriting Logic and Membership Logic}

Maude is a declarative language based on rewriting logic, which has its underlying equational logic as a parameter. In particular, the underlying equational logic chosen for Maude is membership equational logic, a conservative extension of both order-sorted equational logic and partial equational logic with existence equations. It supports partiality, subsort relations, operator overloading, and error specification. 


\section{Membership Logic}

A signature in membership equational logic [144] is a triple $\Omega=(K, \Sigma, S)$ with $K$ a set of kinds, $(K, \Sigma)$ a many-sorted (or "many-kinded") signature, and $S=\left\{S_{k}\right\}_{k \in K}$ a $K$-kinded family of disjoint sets of sorts. The kind of sorts $s$ is denoted as [s]. An $\Omega$-algebra is then a $(K, \Sigma)$-algebra $A$ together with the assignment to each sort $s \in S_{k}$ of a subset $A_{S} \subseteq A_{k}$. A $\Sigma$-algebra contains a set $A_{k}$ for each kind $k \in K$, a function $A_{f}: A_{k_{1}} \times \ldots \times A_{k_{n}} \rightarrow A_{k}$ for each operator $f \in \Sigma_{k_{1} \ldots k_{n}, k}$ and a subset $A_{s} \subseteq A_{k}$ for each sort $s \in S_{k}$, with the meaning that the elements in sorts are well-defined, while elements without a sort are errors. Intuitively, the elements in sorts are the good, or correct, or non-error, or defined, elements, whereas the elements without a sort are error or undefined elements. In general, a total function at the kind level restricts only to a partial function at the level of sorts.

We use $T_{\Sigma}(X)_{k}$ to denote the set of $\Sigma$-terms with kind $k$ over variables in $X$, where $X$ is the set of kinded variables. Atomic formulas are either $\Sigma-$ equations of the form $t=t^{\prime}$, or $\Sigma$-memberships of the form $t: s$, where the term $t, t^{\prime} \in T_{\Sigma}(X)_{k}$ and $s \in S_{k}$. $\Sigma-$ sentences are conditional formulae of the form $\forall X . \phi$ if $\wedge_{i} p_{i}=q_{i} \wedge \wedge_{j} w_{j}: s_{j}$, where $\phi$ is either a $\Sigma$-equation or a $\Sigma$-membership, and all the variables in $\phi, p_{i}, q_{i}$, and $w_{j} \in X$. These sentences are Horn clauses on the atomic formulas, quantified by finite sets of $K$ kinded variables. Such memberships are a generalization of sort constraints and can be used to specify partial functions, that become defined when their arguments satisfy certain equational and membership conditions.

\section{Rewriting Logic}

A Maude system module specifies a rewrite theory [144]. A rewrite theory has sorts, kinds, and operators (perhaps with frozen arguments), and can have three types of statements: equations, memberships, and rules, all of which can be conditional.

A signature in rewriting logic is an equational theory $(\Sigma, E)$, where $\Sigma$ is an equational signature and $E$ is a set of $\Sigma$-equations. Rewriting will operate on equivalence classes of terms modulo $E$. For example, string rewriting is obtained by imposing an associativity 
axiom; multiset rewriting by imposing associativity and commutativity, etc.. Many techniques for rewriting modulo equations have been studied, which is precisely what Maude does, using the equational attributes given in operator declarations - such as associativity, commutativity, and identity - to rewrite modulo such axioms.

Sentences over a signature $(\Sigma, E)$ have the form $[t]_{E} \rightarrow\left[t^{\prime}\right]_{E}$, where $t$ and $t^{\prime}$ are $\Sigma$-terms possibly involving some variables, and $[t]_{E}$ denotes the equivalence class of the term $t$ modulo the equations $E$ (usually, we omit the subscript and simply write $[t]$ ). A rewrite theory $\mathcal{R}$ is a 4-tuple $\mathcal{R}=(\Sigma, E, L, R)$ where $\Sigma$ is an equational signature, $E$ is a set of $\Sigma$-equations, $L$ is a set of labels, and $R$ is a set of labeled rewrite rules with the form of $R \subseteq L \times T_{\Sigma, E}(X)^{2}$ that are either of the unconditional form $r:[t] \rightarrow\left[t^{\prime}\right]$, or of the conditional form $[t] \rightarrow\left[t^{\prime}\right]$ if $\left(\bigwedge_{i} u_{i}=v_{i}\right) \wedge\left(\bigwedge_{j} w_{j}: s_{j}\right) \wedge\left(\bigwedge_{k} p_{k} \rightarrow q_{k}\right)$.

Rewriting logic is a logic for reasoning about concurrent systems having states, and evolving by means of transitions. The signature of a rewrite theory describes a particular structure for the states of a system, and the rewrite rules describe which elementary local transitions are possible in the distributed state. The inference rules of rewriting logic [177] allow to deduce general concurrent transitions which are possible in a system satisfying such a description.

\subsubsection{Functional Module \& System Module}

In Core Maude [48] the basic units are functional modules and system modules. A functional module is an equational style functional program with user-definable syntax in which a number of sorts, their elements, and functions on those sorts are defined. A system module is a declarative style concurrent program with user-definable syntax.

A functional module is declared in Maude using the keywords

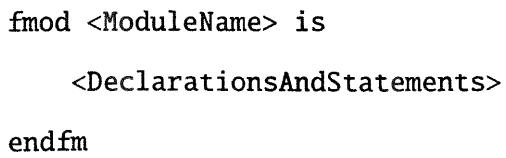


In Maude, functional modules are equational theories in membership equational logic satisfying some additional requirements. Computation in a functional module is accomplished by using the equations as rewrite rules until a canonical form is found. This is the reason why the equations must satisfy the additional requirements of being Church-Rosser, terminating, and sort decreasing.

A system module is declared in Maude using the keywords

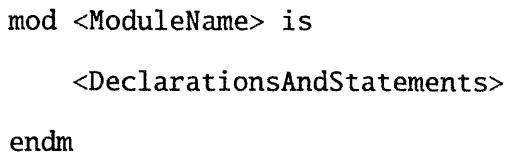

$<$ DeclarationsAndStatements $>$ includes sorts and subsorts, operation, equation, rules, etc.. declaration.

The system modules specify the initial model $\mathcal{T}$ of a rewrite theory $\mathcal{R}=(\Omega, E, L, R)$ in the membership equational logic variant of rewriting logic. These initial models capture nicely the intuitive idea of "rewrite systems" in the sense that they are transition systems whose states are equivalence classes $[t]$ of ground terms modulo the equations $E$ in $\mathcal{R}$, and whose transitions are proofs $\alpha:[t] \rightarrow\left[t^{\prime}\right]$ in rewriting logic, that is concurrent rewriting computations in the system described by the rules in $R$. Such proofs are equated modulo a natural notion of proof equivalence that computationally corresponds to the "true concurrency" of the computations. By adopting a logical instead of a computational perspective, we can alternatively view such models as "logical systems" in which formulas are validly rewritten to other formulas by concurrent rewritings which correspond to proofs for the logic in question.

\subsubsection{Maude Model Checking}

The Maude LTL model checker supports on-the-fly explicit state model checking of concurrent systems expressed as rewrite theories. In the Maude LTL model checker [66] implementation, they introduce a distinguished sort State, the initial model $\mathcal{T}_{\mathcal{R}}$ of a rewrite 
theory $\mathcal{R}=(\Sigma, E, R)$ has an underlying Kripke structure given by the total binary relation extending its one step sequential rewrites. This can be express in the following.

For each kinded term $[t] \in T_{\Sigma / E \text {, State }}$, if we have $[\alpha]:[t] \rightarrow\left[t^{\prime}\right]$ is a proof term in $\mathcal{T}_{\mathcal{R}}$, then $\left[t^{\prime}\right] \in T_{\Sigma / E \text { State. }}$. We can then associate to $\mathcal{R}$ and $S$ tate the Kripke structure $K(\mathcal{R}$, State $)=$ $\left(T_{\Sigma / E \text {,State }}, \rightarrow_{\mathcal{R}}, \mathcal{L}_{\mathcal{R}}\right)$, where $\rightarrow_{\mathcal{R}}$ is the one-step sequential $\mathcal{R}$-rewriting relation on $T_{\Sigma / E, \text { State, }}$, and $\mathcal{L}_{\mathcal{R}}$ is the labeling function with the form $\mathcal{L}_{\mathcal{R}}: T_{\Sigma / \text { EState }} \rightarrow \mathcal{P}(\operatorname{SPred}(\Sigma$, State $))$, where $S$ Pred $(\Sigma$, State $)$ is the set of first order formula.

Maude model checking is an automatic process on a finitary rewrite theory $\mathcal{R}=(\Sigma, E$, $R)$ at initial states $[t]$ of sort State, with the set $\left\{[u] \in T_{\Sigma / E} \mid \mathcal{R} \vdash[t] \rightarrow[u]\right\}$, of all states reachable from $[t]$ is finite. The rewrite theory $\mathcal{R}$ should satisfy the previously mentioned requirement with all reachable states $[u]$ having sort State. Furthermore, the equational theory $(\Sigma, E)$ should be confluent and terminating and the rules $R$ should be coherent relative to the equations $E$ [32]. Note that many rewrite theories of interest may have an infinite number of states, yet the states reachable from any given initial state may still be finite.

Based on the above illustration of the model checking strategy on rewriting theory, we demonstrated the syntactical definition of each model checking module in Maude. A rewrite theory $\mathcal{R}$ satisfying the above assumptions can be specified in Maude by a system module, denoted by $M$. Then, given an initial state, init of sort $S t_{a t}{ }_{M}$, we can model check different LTL properties beginning at this initial state by the following steps:

- defining a new module, say $C H E C K-M$, that includes the modules $\mathrm{M}$ and the predefined module $M O D E L-C H E C K E R$ as submodules;

- giving a subsort declaration, subsort State $_{M}<$ State., where State is one of the key sorts in the module MODEL - CHECKER;

- defining the semantics of the state predicates by means of equations involving the operator

$$
\text { op _ } 1=\text { _ : State Prop } \rightarrow \text { Result [special ...] . }
$$


in MODEL - CHECKER. We define the semantics of each state predicate, say a parameterized state predicate $p$, by giving a set of (conditional) equations of the form:

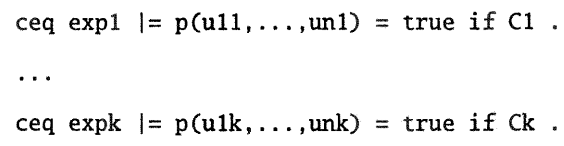

where:

- the expi, $1 \leq i \leq k$, are patterns of sort State $_{M}$, that is, terms, possibly with variables, and involving only constructors, so that any of their instances by simplified ground terms cannot be further simplified;

- the terms $p(u 1 i, \ldots, u n i), 1 \leq i \leq k$ are likewise patterns of sort Prop;

- each condition $C i, 1 \leq i \leq k$, is a conjunction of equalities and memberships; such conditions may involve auxiliary functions, either imported from auxiliary modules, or defined by additional equations in our module $C H E C K-M$.

Once the semantics of each of the state predicates has been defined, we are then ready, given an initial state init, to model check any LTL formula, say form, involving such predicates. This can be done by using the reduce command in Maude as follows:

reduce init $\mid=$ formula .

\subsection{ArchJava Programming Language}

Object oriented programming languages are popular for its object point of view on the program. An object is an entity, either abstract or concrete, which encapsulate the information inside. For the communication to outside, this entity provides an interface that can be invoked by other entities totally or partially. Its attributes generally are invisible so that the 
characters of the entity can be well protected without the malicious attacks or unintended damage.

Although there are many advantages and widely applicable areas of object oriented programming languages, most of object orient programming language is not very good at describing the component-based software architecture. The component building block in the component-based architecture has to be written manually with good care and well design so that it can implement and realize the general function and communicate with environment in a good manner. In order to more efficiently handle the software architecture, which is SAM in our implementation, we used ArchJava as the target programming language in the translation.

ArchJava [19] is an extension to Java that seamlessly unifies software architecture with implementation, using a type system to ensure that the implementation conforms to architectural constraints. In other words, ArchJava is proposed to avoid inconsistency, confusion, and violation of architecture properties when decoupling implementation code from software architecture. Fig. 6 shows a piece of ArchJava code.

\subsubsection{Components, Ports and Architecture Composition}

A component in ArchJava is an object that communicates with other components in a structured way [17]. In ArchJava, a component type class is introduced to specify the type of components that share some behavior and structure.

A component can only communicate with other components at its level in the architecture through explicitly declared ports. A port in ArchJava represents a logical communication channel between two or more components. Ports in ArchJava declare two sets of port modifiers - provides, requires. A provided method is implemented by the component. The definition of a provided method must be given either inside or outside the port. For example (Fig. 7), the search port provides downloadPoem method that can be invoked from the user interface (component SwapUI). The implementation of downloadPoem is shown in Fig. 7. Conversely, each required method is provided by some other component 


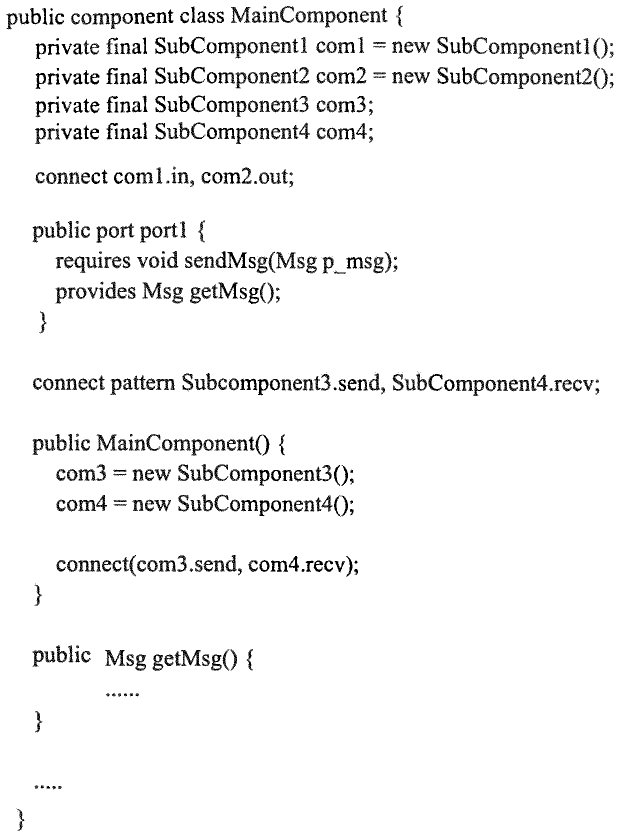

Figure 6: A Shorthand Example of ArchJava

connected to this port. In Fig. 7, the poems port requires method that get descriptions of all the poems in the database (component PoemStroe) getPoem(PoemDesc desc). A port may have both required and provided methods, or have only one or the other as shown in the example.

In ArchJava, a hierarchical software architecture is expressed as a composition of a number of subcomponents connected together. A subcomponent is a component instance nested within another component. For example, in Fig. 8. three subcomponents are a user interface (SwapUI), a poem database (PoemStore), and a poem peer (PoemPeer) instance in the main component PoemSwap.

A static hierarchy of interacting component instances is defined by connect primitive. The connect primitive connects two or more ports together, binding each required method to a provided method with the same name and signature. 


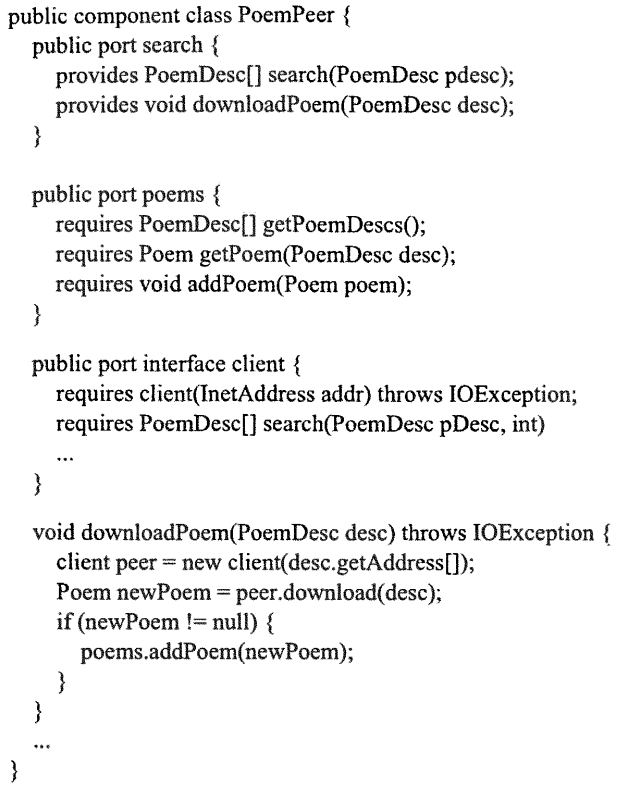

Figure 7: Poempeer class

Connect expression is to describe interaction between dynamically created components at run time. For example, three connect expressions are declared in the class PoemSwap (Fig. 8). A connection pattern is used to document a set of connections that can be instantiated at run time using connect expressions. Each connect expression must match a connection pattern declared in the enclosing component. For example, the first three connect patterns in Fig. 8.

\subsubsection{Connector Abstractions in ArchJava}

Connector [20] in ArchJava is mainly designed for the situation that connectors need to be specified explicitly in large scale systems. The connector class has the ability of type checking and invocation of matched ports in communicated components. User can explicitly define built-in semantics in ArchJava for the invocation of matched ports in a connector. 


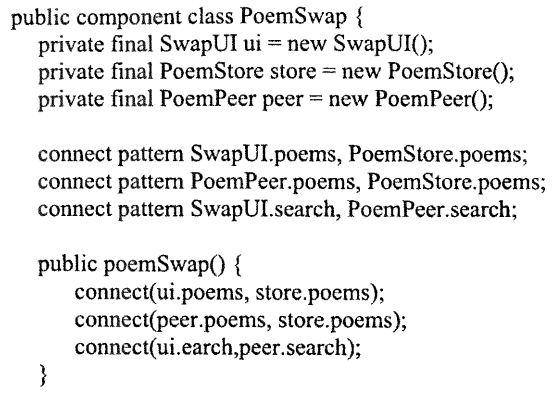

Figure 8: PoemSwap class

\subsection{Aspect Oriented Programming Languages}

Although $\mathrm{OO}$ technology offers greater ability for separation of concerns, it still has difficulty for localizing concerns that do not fit naturally into a single program module, or even several closely related program modules. Concerns can range from high-level notions such as security and quality of services to low-level notions like buffering, caching, and logging. They can also be functional, such as business logics, or non-functional, such as synchronization. Some concerns, such as XML parsing and URL pattern matching, usually couple with a few objects, yet achieve good cohesion. Other concerns, such as logging, will intertwine with many highly unrelated modules.

Aspect-oriented programming (AOP) $[26,118]$ is a new technology for separation of crosscutting concerns into single units called aspects. An aspect is a modular unit of crosscutting implementation. It encapsulates behaviors that affect multiple classes into reusable modules through the pointcut(s) and advice(s). Through this constructs, Aspect-Oriented Programming complements OO programming by allowing the developer to dynamically modify the static $\mathrm{OO}$ model to create a system that can grow to meet new requirements. In other words, it allows us to dynamically modify models or implementations to include code required for secondary requirements (in our case, it is runtime verification) without modifying the original code. Fig. 9(a) is a simple Java class, and Fig. 9(b) shows the aspect 
code that monitors the setting of a point. A pointcut setter () is defined at the method calls either set $X()$ or set $Y()$. Advice after() returning is used to deal with after the method call is returned. For more detailed syntax information, please refer to [26].

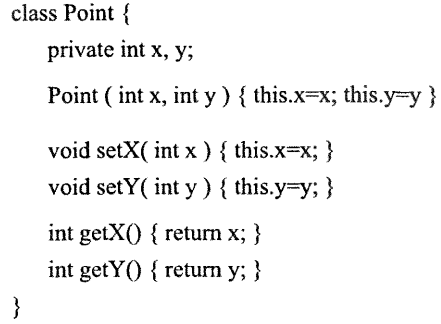

(a) Class Point

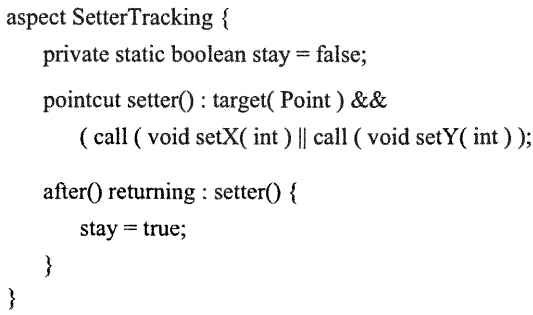

(b) Aspect SetterTracking

Figure 9: An Example of AspectJ

Our runtime checker in the implementation is implemented by AspectJ. Besides, our work shows that an aspect-oriented approach can assist in the generation of component customizations that extend the component with properties declared in the model.

\subsection{Summary}

As we have discussed in the previous chapter, the proposed framework integrates multiple techniques seamlessly: algebraic specifications, Petri nets, category theory, and transformation systems. This chapter gives a brief introduction for each of them as the background knowledge for the following chapters. 


\section{CHAPTER 4}

\section{AN OVERVIEW OF INTEGRATED FRAMEWORK}

\subsection{Introduction}

This chapter describes the basic idea and the structure of an integrated framework for the verification of software architecture model. The basic idea of the integrated framework can be illustrated in a reference frame in Fig. 10.

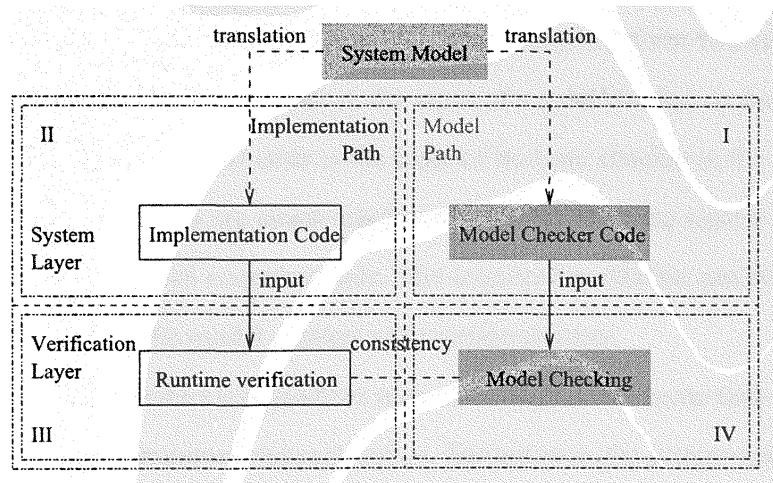

Figure 10: Reference Frame of the Idea

In Fig. 10, each quadrant represents the scope of a technique or data module (holds the file of a model or a program). For instance, the Quadrant I represents the scope of model of the system, the Quadrant IV represents the model checking technique, the Quadrant II represents the program module, and the Quadrant III represents the runtime checking technique. In this reference frame, horizontally there are two layers - system layer and verification layer. System layer (Quadrant I \& II) has the model denoted in the Maude programming language (Quadrant I) and the Java construct code of the model (Quadrant I). Verification layer (Quadrant III \& IV) has the runtime checker (Quadrant IV) and model checker (Quadrant III). Vertically there are two paths - implementation path and model 
path. Model path (Quadrant I \& IV) has the model denoted in the Maude code programming language, and the model checker that is used to check the correctness of the model. Implementation path (Quadrant II \& III) has the Java construct of the model (Quadrant II) that is obtained from a translator, and the runtime checker (Quadrant III) that is used for the property checking of the Java construct. Both the model denoted in the Maude code programming language and Java construct of the model are obtained from the translation on the software architecture model of the system. The architecture model can be viewed as in the third coordinate axis.

The layers and paths with the system model form three types of relations: translation relation, input relation, and consistency relation. The two paths form two input relations - system model in model checker code is the input of model checker in the model path and corresponding Java construct code is the input of runtime checker in the implementation. The translation relations are constructed by translators from the system model to the model checker code and Java construct code. The consistency relation can be obtained by comparing the results of the model checker and runtime checker.

The layers and paths make each quadrant more meaningful. We name each quadrant using a combination of layer names and path names. For instance, the system-model quadrant represents the model is designed and translated into a programming language recognized by a model checker, but has not yet been verified. The system-implementation quadrant represents an individual piece of code translated into Java construct and has not been verified by runtime checker, the Java construct is corresponding to the architecture model. The verification-implementation quadrant denotes the runtime checker that is used to verify and validate some program represented in Java construct. The verification-model quadrant denotes the model checker that is used to verify and validate the model of systems. The above vertical and horizontal relations connect different quadrants together. Next, let us see how to implement each quadrant in the integrated framework. 


\subsection{The Integrated Framework}

The framework [73] is designed on the model checking and runtime analysis of the software architecture model based on the previously illustrated idea. The overall structure of the integrated framework is shown in Fig. 11.

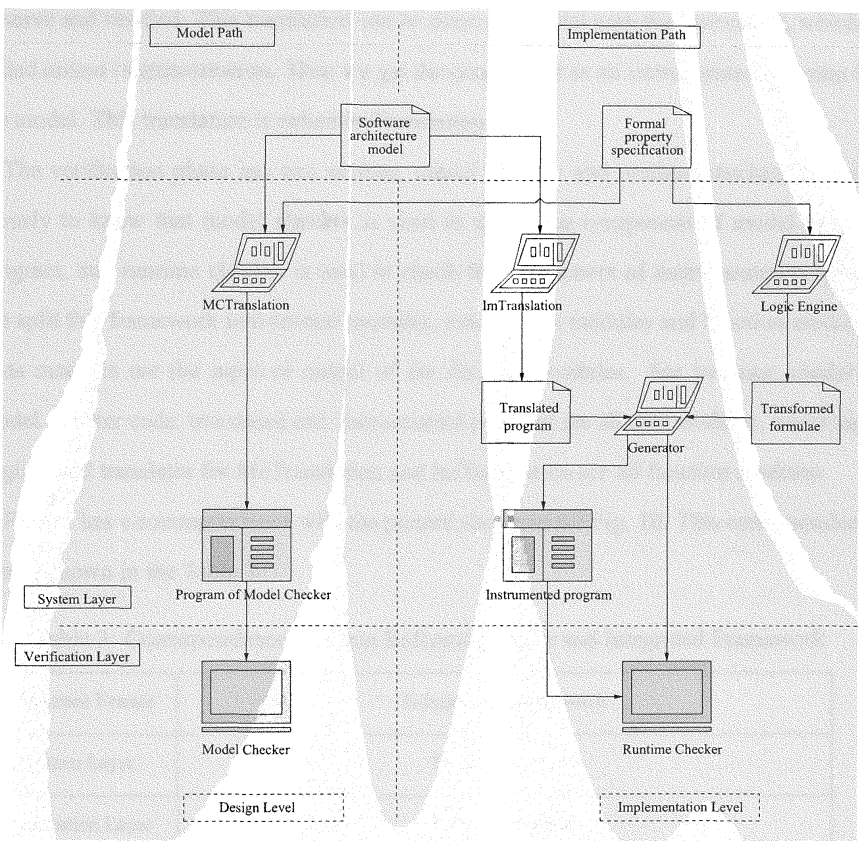

Figure 11: Overview of the Integrated Framework

This framework includes three layers, input layer, translation phase, and verification phase. Input layer has the input file of the software architecture model and formal requirement of the system properties. The translation phase can be split into two parts - model checking parts and runtime checking parts. The model has to be translated into the programming language that recognized by the model checker. This translation outputs the 
component specified by the programming language of model checker. This translation is called MCTranslation. In the runtime checking part the model is translated into the program language in the implementation level. Moreover, it needs to instrument the translated program based on the property specification, so that we can know where and what we should observe and verified. This translation can be done in parallel with the instrument, which is called online instrumentation. Thus we get the component as an instrumented program for the model. This translation is called ImTranslation.

The verification phase has two entities, model checker and runtime checker. It is obviously to know that model checker is used to check the component of model checker program, and runtime checker is used to check the component of instrumented program. We split this framework into several modules, mainly data modules and function modules. Data modules are the input of output of the function modules. For instance, model in model checker code, translated and instrumented program are all data modules, while logic engine, and translator for McTranslation and ImTranslation are all function modules.

Fig. 11 has a correspondence with the picture shown in the Fig. 10. This correspondence can be shown in the Table 3 .

Table 3: Correspondence between Reference Frame and Integrated Framework

\begin{tabular}{|c|c|}
\hline Reference Frame & Integrated Framework \\
\hline System Layer & Translation Phase \\
\hline Verification Layer & Verification Phase \\
\hline Model Path & McTranslation - Program for Model Checker - Model Checker \\
\hline Implementation Path & ImTranslation + Logic Engine - Instrumented Program - Runtime Checker \\
\hline
\end{tabular}

From the Fig. 11, we can see that the model path implements the verification in the design level, and the implementation path realizes the verification in the implementation 
level. Our framework integrates the verification of software architecture model in the design level with that of in the implementation level because of the highly complementary between model checking and runtime verification. The runtime verification is used to resolve the state explosion problem in the model checking. For those properties that cannot be verified due to the size of the system increasing, I can move the verification from design level using model checking to the implementation level using runtime analysis. This results are demonstrated by several case studies in Chapter 8 .

\subsubsection{Input Layer}

The input layer has two parts - Model module $(\mathrm{Mm})$ holds input file of software architecture model, and the Property module ( $\mathrm{Pm}$ ) holds the input file of property specification of the system. Software architecture model is the core part of all data modules in the framework, e.g., all other data modules can be obtained from this module. The model can be any architecture description language that is model-oriented [200]. The reason that I limit the model description language to be model oriented [200] because the model-oriented formal method is constructed in terms of mathematical structures such as tuples, relations, functions, sets, and sequences, thus the mapping strategy can be designed easily from the model to the implementation. On the other hand, I can save the property-oriented description of the system for expected properties. In short,

Model module (Mm) is a set of states $\Sigma=\left\{\sigma \mid \sigma_{1}, \ldots, \sigma_{n}, \ldots\right\}$ that describes the system model $(M)$ behavior using a formal specification language.

Besides, it holds the properties of the system specified formally.

The property module $(\mathrm{Pm})$ is the formatted file for the system property specification. Linear temporal logic (LTL) is used in this work to specify the system properties. These LTL formulae are rewritten into a certain format (e.g., XML file) that can feed to the modules in the translation phase. Since the property module is a transformed specification for system properties, it specifies a set of states that satisfies a proposition $(P)$. In short, 
Property module $(\mathrm{Pm})$ is a set of states $\Sigma^{\prime}=\left\{\mathcal{P}\left(\sigma^{\prime}\right) \mid P\left(\sigma^{\prime}\right) \wedge \sigma^{\prime} \in \Sigma^{\prime}\right\}$, where $\mathcal{P}\left(\sigma^{\prime}\right)$ is a power set of state $\sigma^{\prime}, P\left(\sigma^{\prime}\right)$ denotes property $P$ holds on the state $\sigma$.

We have $\Sigma^{\prime} \subseteq \Sigma$.

\subsubsection{Translation Phase}

The translation phase has two parts - McTranslation and ImTranslation. McTranslation module translates the architecture Model module to the program that is recognized by a model checker. Let $P m$ represent the data for the program recognized by a model checker. The McTranslation defines a mapping relation $\left(T_{M c}\right)$ from the Model module $(\mathrm{Mm})$ to the program module recognized by a model checker $(P m)$, e.g., $T_{M c}: M m \rightarrow P m$. ImTranslation module translates architecture Model module to the low level programming language in the implementation level (in this case I use object oriented programming language (OOPL)) to feed to the runtime checker. Let $P j$ denote the data module specified by the object oriented programming language. ImTranslation implements a mapping relation $T_{I m}$ from Model module $(\mathrm{Mm})$ to the $\mathrm{OO}$ programming language data module $(P j)$. In short, $T_{I m}: M m \rightarrow P j$. The translation algorithms are demonstrated in the following two chapters: Chapter 5 and Chapter 6.

Currently there are several different model checker tools developed during recent decades, such as Maude, SMC, SPIN, etc.. In this dissertation, I did not develop a new model checking tool. Ichoose one available tool for the model checking purpose since develop a model checker is not the research goal. Model checking (Mc) is an exhaustive searching process of the property states out of all system states, e.g., $M c=\left\{\forall \sigma_{i} \in \Sigma . \cup_{i} \sigma_{i}=\right.$ $\left.\Sigma^{\prime}\right\}$.

Runtime checker module $(R v)$ has two basic functions - discharge the necessary events for the model monitoring as soon as possible, and check the event traces against the properties specification. In short,

$R v=\left\{\forall \sigma_{i} \in \Sigma_{\text {curr }} \cup_{i} \sigma_{i}=\Sigma^{\prime}\right\}$, where $\Sigma_{\text {curr }}$ is the currents system states that the model is executing, $\Sigma_{\text {curr }} \subseteq \Sigma$. 


\subsubsection{Translation Validation}

There are two translations in the integrated framework. The verification results on the model highly depend on the correctness of the translations. If the translation is not correct, it does not make sense to verify the system model because the verification is not reliable for the model. Several works have been done for the correctness proof of the translation from a formal model to the model checker program using category theory $[65,162,163$, 168]. In Chapter 7 I proposed methods to formally prove the behavioral consistency for the translation from SAM architecture model to the Maude programming language [47].

The correctness proof from the formal model to the low level implementation program is still a very challenging research issue. In Chapter 7, I proposed a semantic-based method to prove the behavior consistency between the SAM model and the Java construct output from the runtime checker SAM Parser by stepwise comparison of the state sequence.

\subsubsection{Verification Phase}

In the verification Phase there are two purposes - verify the system properties of the model in the design level and in the implementation level. In this work, I choose some available model checking tool (Maude [47]) to verify the system model in the design level.

The discussion of the design level verification using model checking is presented in Chapter 5.

In the implementation level, the primary objective was to create a runtime environment that would simplify the realization, implementation, execution, and monitoring of SAM architectures while preserving the properties specified in the model. To develop a methodology to reach this goal, I (1) identify an automatic implementation of SAM models, (2) generate runtime verification code from SAM properties, and (3) instrument or weave the runtime verification code into the implementation.

The discussion of the implementation level verification is presented in Chapter 6 . 


\section{CHAPTER 5}

\section{VERIFICATION AND VALIDATION IN THE DESIGN}

\subsection{Introduction}

A software architecture (SA) design provides a high-level abstraction of system topology, functionality, and/or behavior $[167,187]$, which provides a basis for early system understanding and analysis as well as a foundation for subsequent detailed design and implementation. Therefore, software architecture plays a critical role in the software development process. In the past decades, tremendous research has been done on software description languages and their analysis.

There are two main research groups in the field of software architectures analysis: one group has focused on the design issues, formal foundations, and analysis of architectures, which is discussed in this chapter; while the other has resulted in technologies for implementing software architectures, which is discussed in the Chapter 6. The first approach has focused on architectural design abstractions called styles and the semantics underpinning such styles [187]. To date theorem proving and model checking are two main streams for the architectural analysis. As discussed in Chapter 2, model checking is widely used in academic and industrial for the software analysis and verification due to its highly automatic feature and counter example feedback.

Model checking is a technique building a finite state model of a system and checking whether a given property holds in that model [44]. It designates a collection of techniques for the automatic analysis of reactive system. The inputs to a model checker are a (usually finite-state) description of the system and a number of properties. The model checker either confirms that the properties hold or reports that they are violated by giving an counter example. There are several different model checking tools, such SMV $[113,138]$, SPIN $[45,121]$, etc.. The Maude model checker supports on-the-fly explicit state model checking 
of concurrent systems expressed as rewrite theories. We introduce the related work on architecture analysis in the following section.

\subsection{Related Work}

Analyzing software architecture is to predict the quality of a system before it has been built and not to establish precise estimates but the principal effects of an architecture. Various architecture analysis methods have been proposed in the literature. An overview of scenario-based architecture analysis method is given [54]. Scenario-based architecture analysis method $[116,117]$ appeared in 1993 , whose purpose is to develop a systematic way to evaluate quality attributes on the model-based architecture. Correctness is one of the key attributes of the software product quality. The correctness verification is infused into the several system key properties, such as safety, functional requirements analysis methodology $[89,90,94,95]$. Our work is to develop an integrated and systematic methodology for the correctness assurance based on the integration of the formal analysis methods - namely, model checking and runtime verification. In this chapter, we focus on the model checking based architecture analysis and verification method for the correctness assurance.

\subsection{Software Architecture Verification \& Analysis}

In this section we discuss the software architecture verification \& analysis in the design level. First, we give an architecture-independent software architecture verification \& analysis method (SAVAM). Then based on the method, we present the application of SAVAM on the Software Architecture Model (SAM).

\subsubsection{Software Architecture Verification \& Analysis Method}

Software architecture provides a high level abstraction of large scale complex systems in the design level. However, the verification and analysis of software systems lag far behind the increasing growth in size and complexity of software systems. This is due to the fact that methods, techniques and tools for assuring the software quality, e.g,. correctness, reliability, etc., fall behind the software modeling and specification. In this section, we 
address a model checking based, architecture model independent verification and analysis method with respect to software quality, especially, correctness. This method can be mainly enumerated below.

1. Identify the software system using specific software architecture description language.

2. Characterize a canonical functional partitioning for the domain.

3. Map the functional partitioning onto the architecture's structural decomposition.

4. Choose a (set of) quality attribute(s) (in our work is correctness) with which to assess the architecture.

5. Choose a proper model checker as the verification and analysis tool.

6. Map the software model onto the programming langauge recognized by the model checker.

7. Identify a proper set of structure properties from the architecture structure.

8. Identify a proper set of behavior properties from the architecture behavior.

9. Map the software architecture properties to the specification specified by the model checker.

10. Evaluate the architecture model of the system against the properties using model checker.

11. Analyze the results.

In our work, as an example of the software architecture verification and analysis method (SAVAM), we chose SAM as a software architecture model, and Maude as the model checker. In the application of SAVAM on the SAM architecture model and Maude model checker, we have instantiate the above procedure as follows. 
1. Identify the software system using SAM architecture model.

2. Characterize a canonical functional partitioning as components, connectors for the domain.

3. Map components and connectors onto the architecture's structural decomposition.

4. In our work, we focus on the correctness verification. Correctness is one of the features of the software product qualities.

5. Choose Maude model checker as the verification and analysis tool.

6. Map the SAM model onto the Maude programming langauge. This procedure is provided in the next section in detail. The validation of the translation is provided in the Chapter 7.

7. Identify a proper set of structure properties from the SAM architecture structure.

8. Identify a proper set of behavior properties from the SAM architecture behavior.

9. Map the software architecture properties to the specification specified by the Maude model checker.

10. Evaluate the architecture model of the system against the properties using Maude model checker. The results are demonstrated in several case studies in Chapter 8 .

\subsubsection{Software Architecture Perspectives}

The architectural design of a software system can be described from (at least) three perspectives - the behavior of its domain of interest, its structure, and the property classification on the architecture domain. These perspectives reflect a consensus within the software architecture community, as witnessed by the literature [142]. We will discuss each of these perspectives in turn. 


\section{Behavior - Functionality}

A system's functionality is what the system can do during the execution. It may be a single function or a bundle of related functions which together describe the system's overall behavior. For large systems, a partitioning divides the behavior into a collection of functions which together comprise the system's function but which are individually simple to describe or otherwise conceptualize. Typically, a single system's functionality is decomposed through techniques such as structured analysis [143] or object oriented analysis [178], but this is not always the case. For the software product quality analysis, such as correctness, a model based approach will bring more accurate and precise reasoning of the system. An important feature of software architectures is the ability to allow reasoning on interesting properties of the system. This would allow an early in the software development analysis of relevant features of the system, and it would help in evaluating the suitability of a software architecture. Automatical analysis of the software architecture model with tool support can further highly reduce the errors introduced by human involvement. Several works have been done in formal analysis and tool support for the architecture quality analysis $[98,203]$.

\section{Structure}

A system's structure is the combination of ingredients of the system abstraction, which reveals how it is constructed from smaller connected pieces. The structure is described in terms of the following parts: 1 . A collection of components which represent computational entities (e.g., a process) or persistent data repositories (e.g., a file); 2. A representation of the connections between the components, that is, the communication and control relationships among the components. In our analysis of architectures, we use notations in software architecture model SAM - a component based formal architecture model as lexicon.

To understand the overall behavior of a system, we would need to provide more detailed descriptions of the computations possible within components and the overall coordination of a collection of components with various connection relationships between them. Such 
computational and coordination models are explicit in a SAM architectural description model.

\section{Property Classification}

Property classification is one of the key issue for the software architecture verification and analysis due to the following reasons. First, the verification of system is based on the architecture model against the system properties. Without property specification, there is no way to put the verification. Second, system behaviors are specified on the functional and nonfunctional features, which are formalized in the property specification of the system. Thus the formal verification techniques, such as model checking, can be applied. In our work, system properties are specified as temporal logic formula. Although the main two streams of the temporal properties are safety and liveness, several works have been done for the temporal logic property classification.

Safety liveness properties are first proposed by and stipulated in the work of [123]. The first formal definition of safety and liveness was given by Alpern and Schneider [22]. In contrast to Lamport, they represented a finite prefix of an execution as the set of all possible continuations from that point on, which leads to a slightly more general notion of safety properties. A property is a liveness property, if and only if it contains at least one continuation for every finite prefix. This corresponds to the intuition that the "good thing" can still happen after any finite execution.

The difference between this two characterization is in those properties that are not invariant under stuttering. However, an explicit use of finite executions for the characterization settle the matter.

Further distinct work on safety-liveness property characterization was presented by Zohar and Amir in their work of [135]. In this paper [135], the property is characterized as a hierarchical organization mainly being composed of safety and liveness properties. The combination of these two basic property categories, four more complicated categories are derived. The hierarchical organization is shown in the figure Fig. 12. 


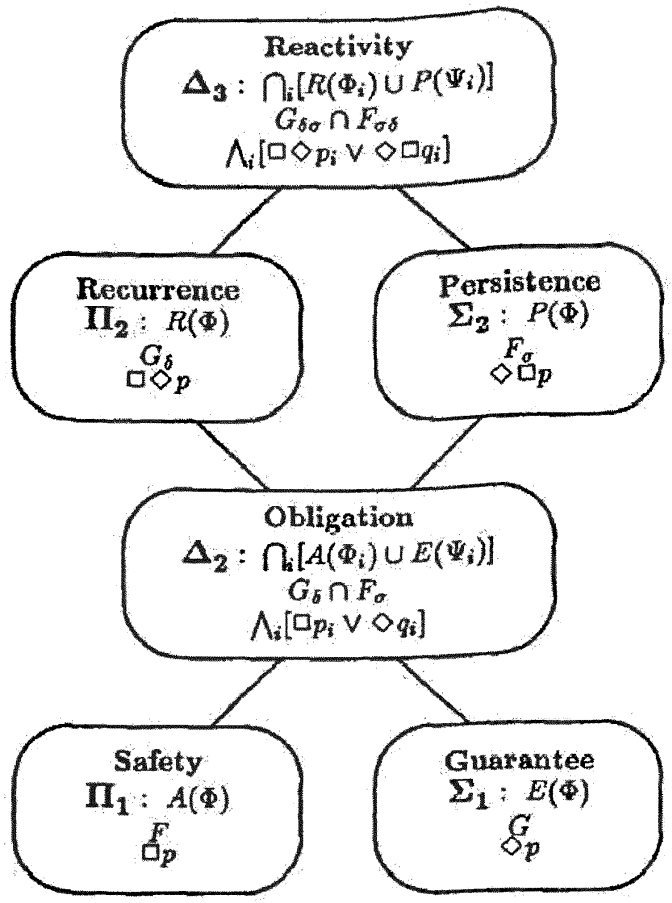

Figure 12: Property Hierarchy

In this hierarchy, the upper level contains properties in the lover level, i.e., properties in the lower level is a proper set of those in the upper level. This hierarchical classification is based on the investigation of finite words or sequence of states. Properties of property classes in the hierarchy are

1. Closure: (using set operations)

safety, guarantee properties - closure under union and intersection;

persistence, recurrence properties - closed under union and intersection;

obligation is closed under three boolean operations;

reactivity is closed under three boolean operations. 
2. Duality: safety under complement is liveness properties; recurrence under complement is persistent properties; and vice versa.

3. Topological properties: safety properties are the closed sets and liveness properties are the open sets (This coincides with [22].); recurrence properties are the $G_{\delta}$ sets and persistent properties are the $G_{\sigma}$ sets.

4. Every property can be represented as the intersection of safety and liveness property.

5. Semantical relation: Every specifiable k-property is a $\mathrm{k}$-specifiable property.

All these properties hold for the four characterizations: language view, topological view, temporal logic view, automata view. Finally, the conclusion from [135] is coherent with that in [22] (for those invariant on stuttering). Further work is [38]. They do not characterize a partition but a hierarchy of properties. The least level of this hierarchy coincides with the characterization of safety properties. The higher levels provide a finer distinction of different liveness properties.

[155] extended the safety-liveness classification on the finite state sequence for a computation to the infinite state sequence for all computations. I think, this can fall in the group of CTL classification.

A different kind but practical classification based on the system context was proposed by [59].

All these classifications fall in the safety-liveness point of view. Moreover, few of them considered the complexity of model checking on different classes. The work of Ivana and Radek [37] investigated the complexity based on the classification in [135], thus filled in the piece of blank in this area.

In [37], Ivana and Radek pointed out for explicit model checkers, there is no complexity difference for different classes in the hierarchy for all algorithms presented. Some benefits can be obtained by employing some heuristics on different algorithms. While it's more efficient to use specialized algorithm for symbolic model checkers. Thus properties are 
classified in terms of the verification complexity as three classes: reactivity, recurrence and safety from difficulty to easy.

The work [59] provided a thorough overview on the property classification patterns and also discussed the mappings to LTL formulas. These patterns are abstracted from over 500 examples. The authors also created an online repository for information about property specification for finite-state verification, and keep updating the property patterns. The property patterns identified in the work [59] are general to many systems, but some are not suitable for specific domains. In the following, we will identify some of the properties in the SAM architecture model.

To be consistent with the SAM architecture specification, we identify the properties as two main streams in SAM - structure properties and behavior properties. Besides that, since SAM is also suitable for the realtime embedded systems, the realtime properties are listed. Because realtime system is out of this dissertation, we do not consider this group in detail.

1. Structure Properties. This kind of properties is closely related to the topology of the model. Most of these properties can be directly verified on the SAM model without animating the system. These properties are necessary conditions that ensure the feasibility of the system. If one of them is not fulfilled, we can assert firmly that the system is erroneous. Among these properties, we cite the following properties:

- Port consistency. The port $p_{1}$ for a component/connector has to be semantically consistent with the port $p_{2}$ that communicates with it of a connector/component. The semantically consistency means that 1 ) the two ports have to have the same name; 2) the token (tk) flowing out of one port flows into another port. This property has the following pattern: $\square\left(p_{1}(t k) \rightarrow p_{2}(t k)\right)$.

- Token flow. For a component/connector, if a token (tk) is consumed by the transition after the input port $\left(p_{i}\right)$, and then the same token (tk) is produced by 
the transition before some output port $\left(p_{o}\right)$. The property for the token flowing has the pattern: $\square\left(p_{i}(t k) \rightarrow \diamond p_{o}(t k)\right)$

- Sort consistency. If there is token flowing through a component/connector, the sorts for the input and output have to be consistent, this can be validated through the guard condition defined in the transitions.

- Structure splitting. One component $\left(C_{m 1}\right)$ can communicate more than one components $\left(C_{m i}\right)$ through one connector $\left(C_{n}\right)$. The communicated ports in the other components $\left(C_{m i}\right)$ have to follow sort consistency, sometimes may also need to follow token flowing.

Those enumerated structure properties are easily to be verified. Many of them can be observed through the structure or the guard in the transitions.

2. Behavior Properties. This kind of properties concern the dynamic behavior of the systems. This means that they are related to state changing of the system. The evaluation of the dynamic behavior properties are based on the behavior description - Petri nets. Its verification is achieved on a set of places describing a possible evolution of the system. Among these properties, we cite the following properties:

- Responsiveness. This property indicates for any request sent from an output port $\left(p_{o}\right)$ in a component, there must be a response from an input port $\left(p_{i}\right)$ in another component. The token information may be different. The pattern for this property is: $\square\left(p_{o}\left(t k_{o}\right) \rightarrow \diamond p_{i}\left(t k_{i}\right)\right)$.

- Selective responsiveness. The receiving ports $\left(p_{s}\right)$ get information from the same sending ports $\left(p_{r}\right)$, and the relation between two receiving ports $\left(p_{s 1}, p_{s 2}\right)$ are exclusive or. The pattern for this property is: $\square\left(p_{r} \rightarrow\left(p_{s 1} \vee p_{s 2}\right)\right)$. Here we use or to represent exclusive or.

- Conjoined reverse. This property provide a reverse reasoning from the input port in a component, generally it has to be past time LTL. 
- Cause-effect. This property expresses a chain relation among more than one component. The ports involved have to be in an ordered relation. If the ordered relation is broken, the property cannot hold.

3. Realtime Properties. These properties aim at evaluating the system in terms of time response. Having constraints on the different activities, we want to be able to determine if the system can answer in a limited time. The constraints that can be stated on activities include delay, period, deadline, etc.

Most of the behavior properties can be referred to the work [72].

In the verification and analysis process, we concern mainly the behavior properties, since structure properties are easily to be identified and verified. The results for the property verification will be discussed for each case study in the Chapter 8 . Next, we discuss the translation algorithm from SAM to Maude model checker in order to fulfill the requirement of the verification and analysis method using Maude.

\subsection{Translation from SAM to Maude}

The SAM model allows formal validation of a component system against system constraints and property specified on its abstraction represented by a component or connector. Here, validation means that the developer can animate the specification by providing initial markings and checking if the responses meet the expected results. Validation of SAM is based on the precise syntax and semantics of Petri net formal language and temporal logic. The validation will cover the topology and dynamic behavior of the Petri net as well as temporal logic formulae. Here we present how to translate SAM model to the Maude language.

Step 1. Translation to the functional module: generate the sorts operators used in the functional modules for the model signatures. This step translates each place, sorts, markings in a Petri net into the corresponding part in Maude's functional module.

1. For all sorts in the behavior model. 
- Int, bool and String sort in a Petri net are also sorts in Maude. Int and bool are implicitly imported while String module must be imported by user explicitly.

- Other user defined sorts that are not defined in Maude must be declared as sorts in the functional module.

- Declare a sort named Marking.

2. For all ports in a component/connector and places in the behavior model.

- Map them to the operator $p$ with the same identifiers, and we have the following sentence in function module:

op p : <sorts> -> Marking .

- Note: each element in the Cartesian product is a parameter in the operator.

Step 2. Translation to the system modules. There are three types of system modules, one is for the model signature that corresponds to the architecture structure and dynamic behavior of the model, one is for the mapping to the predicates, and one is for the model checking, which includes the property specification.

1. Model structure and dynamic behavior Module (SysID).

- Define each basic component or connector as a system module with the declaration of variables and necessary rules and operators.

- Each composition is specified as a system module that including its subcomponents and connector that are predefined as a module.

- All guard conditions in a transition are a (un)conditional rule with the following format:

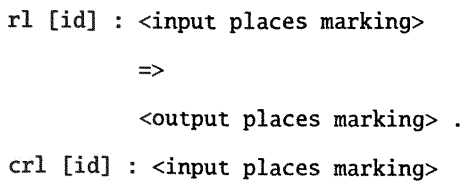




$$
\begin{aligned}
& \Rightarrow \\
& \text { <output places marking> } \\
& \text { if <condition marking>. }
\end{aligned}
$$

where $i d$ is the identifier of the transition.

2. Predicates mapping module(SysID-PREDS). In this module each place is mapped to an operator in Maude.

- The identifier of an operator is $P$ connected with port or place's identifier.

- The parameters of an operator are the sorts of the place.

- Result type of this operation is predefined type for logic formula in Maude (Prop).

- Finally, we have to connect each operator with a predicate by defining equations as following format:

$$
\text { eq } \mathrm{m} 1 \text { op } \mathrm{m} 2 \quad \mathrm{l}=\mathrm{Pid}=\text { true } \text {. }
$$

where $m 1$ and $m 2$ are Markings.

3. Property specification module (SysID-CHECK). This is the model checking part.

- Define initial marking and property identifiers as constant operators with the following formats:

$$
\begin{aligned}
& \text { op init : } \rightarrow \text { Marking . } \\
& \text { op property : } \rightarrow \text { Formula . }
\end{aligned}
$$

- Each initial marking and property are specified as equations with following formats:

$$
\begin{aligned}
& \text { eq init }=<\text { initial markings }>\text {. } \\
& \text { eq property }=\langle\text { predicates }>\text {. }
\end{aligned}
$$


Each module lately created must import necessary modules predefined by user or in Maude to reuse those specifications or program. The rules in the structure and behavior module (SysID) need to use operators defined in the model signature functional module so the functional module (SysID) is imported in the structure and behavior module. The predicate system module (SysID-PREDS) use sort State and logic operations predefined in the Maude so functional module Satisfaction was imported. This module also use signature functional module (SysId) so the structure and behavior module (SysID-PREDS) is imported. Finally property specification and checking module (SysID-CHECK) imports predicates module (SysID-PREDS) and model checking module MODEL-CHECKER and linear temporal logic module $L T L$-SIMPLIFIER.

In our translation, system signature such as sorts and operators are declared in the functional module. This translates the places/ports, sorts into algebra in Maude that will be used in the system modules. The dynamic semantics of Petri net can be mapped to the rewriting rules used in Maude. Computationally, the meaning of rewriting rules is to specify local concurrent transitions that can take place in a system if the pattern in the rule's lefthand side matches a fragment of the system state and the rule's condition is satisfied. In that case, the transition specified by the rule can take place, and the matched fragment of the state is transformed into the corresponding instance of the righthand side. Thus we can see an amazing match between semantics of Petri net and rewriting logic. These are theoretic aspect of the above translation algorithm.

The underlying theoretical background can be explained as follows. A rewrite specification $\mathcal{R}$ consists of a single-sorted signature $\Omega_{\mathcal{R}}$, a set $E_{\mathcal{R}}$ of equations over $\Omega_{\mathcal{R}}$, and a set $R_{\mathcal{R}}$ or labeled rewrite rules. A rewrite $P: M \rightarrow N$ means that the term $M$ rewrites to the term $N$ modulo $E_{\mathcal{R}}$, and this rewrite is witnessed by the proof term $P$. Apart from general (concurrent) rewrites $P: M \rightarrow N$ that are generated from identity and atomic rewrites by parallel and sequential composition, rewriting logic classifies its most basic rewrites as follow: a one-step (concurrent) rewrite is generated by parallel composition from identity and 
atomic rewrites and contains at least one atomic rewrite, and a one-step sequential rewrite is a one-step rewrite containing exactly one atomic rewrite.

The operational semantics of Rewrite specification extends the operational semantics of membership equational specification by applying computational equations $E_{\mathcal{R}}$ and rewrite rules $R_{\mathcal{R}}$ modulo the structural equations $E_{\mathcal{R}}$. The process net for the behavior model Petri nets - can be matched into the rewrite theory R. For instance, signatures and rewrite rules can be mapped into the net specification and places, and transitions with their preand post-sets. Instead of using operational semantics, the work [144] demonstrated the isomorphism between category Petri nets and category Rewrite theory.

\subsection{Summary}

This chapter demonstrated a model checking based software architecture verification and validation method. The method is established on a general picture for the software architecture verification and analysis method (SAVAM), and composed of several key steps. In our work, the model we choose is SAM software architecture model, the model checker is Maude model checker. Based on the SAVAM, we provided a systematic translation algorithm from SAM to Maude programming language. The background theory is rewriting logic and membership equation logic.

With the property specified in the LTL, we classified properties in SAM architecture into three main streams, two of them are discussed. The last group of properties is out of scope of this dissertation, we skip it as future works. 


\section{CHAPTER 6 \\ VERIFICATION \& VALIDATION IN IMPLEMENTATION}

\subsection{Introduction}

To date, most architectural tools have focused on the simulation and analysis of architectural models to exploit the semantic power of ADLs. At the same time, insufficient progress has been made on supporting implementation of applications based on styles and ADL models [142]. However, analysis of software architecture is not enough to generate correct product due to two reasons. On one hand, not all properties can be verified at design level because of the state space explosion problem, verification costs and characteristics of open-system. On the other hand, a correct and valid software architecture design does not ensure a correct implementation due to the error-prone characteristics of the software development process.

As discussed in Chapter 5, the group focuse on providing software frameworks, often through object-oriented reuse techniques such as design patterns and object hierarchies. This approach has lead to creation of a variety of mediate techniques and associated commercial technologies for component-based development $[14,185,199]$. However, software implementations resulting from such use of frameworks often differ widely from conceptual models and lack adequate semantic underpinnings for analytic purposes.

\subsection{Related Works}

We can identify in the literature by two categories of works that are mostly related to our research. The first one concerns the works that implementing software architecture descriptions automatically without considering the software correctness and software product quality after release. The second one is composed of the works generating and weaving runtime verification code into the implementation. 
Focusing on the first category, several $\mathrm{ADL}$ tools such as MetaH and $\mathrm{C} 2$ support code generation that translates an $\mathrm{ADL}$ to a programming language with various strategies. In Darwin [81], components, portals, and bindings were implemented as ordinary Java classes and methods. The communication between component instances were designed as the client-server pattern through message passing using Java RMI (Java Remote Method Invocation) mechanism. C2DRADEL [141], the development environment for Chiron-2 (C2) architecture, was one of the successful works of Java or $\mathrm{C}++$ code generation from software architectures. It provided both code generation and system analysis mechanisms. MetaH [197] toolkit was designed to generate Ada code and provided system analysis function modules such as schedulability and reliability etc. Unicon [186] and Weaves [87] used "glue code" to integrate high level description with implementation. However, they cannot enforce communication integrity $[131,152]$ in the implementation that is necessary to enable architectural reasoning about an implementation [19]. This is the main reason to choose ArchJava as the target language in our work.

Considering the second category, among the existing works on runtime verification, $\mathrm{MaC}[126]$ is the closest one to ours. $\mathrm{MaC}$ framework needs several inputs from users: a monitoring script in PEDL that provides a mapping between high-level events used in the requirement specification and low-level state information, a requirement specification in MEDL that define properties in a special interval logic, and a system implementation. Runtime checker generated from the requirement specification evaluates requirements over the current event trace received from the event recognizer. Our work can be viewed as a special case of $\mathrm{MaC}$ on software architecture descriptions, more specifically SAM models. Therefore, we can obtain more benefits in terms of automation. In our work, monitoring script and requirement specification is not necessary since they are either implicit or explicit expressed in SAM models. Further more, the system implementation of SAM models is also generated automatically. Unlike MaC framework, runtime verification systems such as JPAX $[92,95]$ currently support linear temporal logic, and some analysis algorithms and deadlock detection algorithm. Currently, our work does not implement such 
algorithms. However we extend the range of properties to be verified: a subset of first order linear temporal logic formulae. Although this subset looks small, it actually covers most of SAM properties such as response properties involving quantifiers. Monitoring Oriented Programming (MOP) [39] shows a different way to implement runtime monitoring, where monitoring code is synthesized automatically from the requirement logic annotations before compilation and inserted into the appropriate places, it supports both on-line and off-line monitoring. However, MOP requires that software developers have a deep understanding of the code to catch all "critical" places manually, which is the issue we want to avoid.

\subsection{System Architecture}

Our primary objective was to create a runtime environment that would simplify the realization, implementation, execution, and monitoring of SAM architectures while preserving the properties specified in the model. To develop a methodology to reach this goal, we (1) identify an automatic implementation of SAM models, (2) generate runtime verification code from SAM properties, and (3) instrument or weave the runtime verification code into the implementation.

Fig. 13 is the overview of the structure of our methodology. Our method has three levels: design level, tool support level and implementation level, in terms of data flowing.

Design level handles the SAM model and its input format. Two different types of input are specified: SAM_XML and PNML. SAM models are expressed in an XML-based interchange format (e.g. SAM XML), which specifies the SAM structure, property specifications for components or connectors. Behavior model (Petri nets) are defined as PNML (Petri Net Markup Language) [30] files, which specify Petri nets in an XML-based interchange format. Although SAM supports any temporal logic and any kinds of Petri nets, here only linear temporal logic and high level Petri nets are considered.

SAM Parser and the logic server, the tools we developed, fall in the tool support level. The purpose of SAM Parser is to take the design level as input, by communicating through 
logic server and generate the function and monitoring code to be executed. This level will be discussed in detail in the next subsection. All generated code forms the implementation level. They work together for an integrated system of architecture realization and runtime monitoring. Their output results, the runtime verification of architecture model against system properties in the implementation level, are used to analyze the architecture implementation and guide the application of the system design.

\subsection{Translation from SAM to Java Construct}

There is a growing awareness, both industry and academia, of the crucial role of formally proving the correctness of safety-critical portions of systems. Most verification methods focus on the verification of a design against requirements, and high-level code with respect to a given design specification. However, if one is to prove that the high-level specification is correctly implemented in low-level code, one needs to verify the translation which performs the conversion from high-level specification to the low-level code. Verifying the correctness of translation is challenging because of the complexity and syntactic and semantic diversity of the target architectures, as well as the sophisticated analysis and optimization algorithms used in the process of translation. This is discussed in the next chapter.

Formally verifying a full-fledged general translation from a high-level specification to an object oriented programming language is not feasible, due to its size, variety of source and target languages, and, possibly, proprietary considerations. This dissertation proposed an approach to validating the translation used in the SAM Parser, a runtime analysis tool that verifies the high-level architecture specification SAM in the implementation.

\subsubsection{Architecture Structure Translation}

Architecture structure includes the entities: compositions, components, connectors, ports, which are mapped into the corresponding entities in ArchJava. The mapping relation is shown in the following Table 4.

From the Table 4., we can see that there is a one-to-one mapping from the elements of SAM architecture structure to entities in the ArchJava programming language. Although 
Table 4: Mapping relation between architecture structure and ArchJava

\begin{tabular}{|c|c|c|c|}
\hline \multicolumn{2}{|r|}{ SAM } & \multicolumn{2}{|l|}{ ArchJava } \\
\hline \multicolumn{2}{|c|}{ Composition C } & \multicolumn{2}{|l|}{ Component Class } \\
\hline \multicolumn{2}{|c|}{ Component $\mathrm{Cm}$} & \multicolumn{2}{|l|}{ Component Class } \\
\hline \multicolumn{2}{|c|}{ Connector $\mathrm{Cn}$} & \multicolumn{2}{|l|}{ Component Class } \\
\hline \multirow{4}{*}{ Port } & Incoming Port & provides method within port modifier & \multirow{4}{*}{ port } \\
\hline & Outgoing Port & \multirow{2}{*}{$\begin{array}{l}\text { requires method within port modifier } \\
\text { connect pattern }\end{array}$} & \\
\hline & Glued Port & & \\
\hline & Connected Port & connect pattern & \\
\hline & Tokens & Java Classes & \\
\hline
\end{tabular}

all composition, component, connector are mapped to component class in ArchJava, for different concrete element in SAM Architecture structure, there is a corresponding component class after the translation.

In the ArchJava code, there is no structural difference between composition, and compound components. Since the components with the subcomponents are still compositions, this translation is consistent with the definition in SAM specification. Secondly, there is no difference between the translated component and connector since both are translated into component classes in ArchJava. The difference exists whether or not the translated component class is a flattened component/connector. In SAM specification, the component and connector share the same architecture structure and behavior model definition. So this translation design is consistent with the definition in SAM specification. Finally, the only difference existed between translated codes of components is whether or not the component is a flattened component.

Component class is the most important class in the ArchJava code since each component/connector in SAM has to have and only one component class. The system is started when one of the component class is triggered and run. The implementation of Component class is shown in the Table 5. Each component class is implemented as a thread, thus each component class instance is self activated. When it is activated, a Net object is generated for its behavior model - Petri net. Its behavior is registered which indicates the component instance is a flat component in SAM. 
Table 5: Component Class definition in ArchJava

\begin{tabular}{|l|l|l|}
\hline \hline & FIELDS & METHODS \\
\hline Component Class & $\begin{array}{l}\text { Subcomponent declaration; } \\
\text { Behavior behavior; }\end{array}$ & $\begin{array}{l}\text { public Constructors f } \\
\text { Connect); } \\
\text { Subcomponents.add(); } \\
\text { Nubcomponents.start); /for flat component } \\
\text { Register behavior; } \\
\text { connect patterns for all ports. } \\
\text { ports declaration; } \\
\text { private sendMessageFromPort); } \\
\text { public run();/for flat component }\end{array}$ \\
\hline Port declaration & $\begin{array}{l}\text { requires/provides port interface } \\
\text { provides port method im- } \\
\text { plementation }\end{array}$ & $\begin{array}{l}\text { public addMessage(String pt, Object msg) } \\
\text { public recvMessage(String pt, Object msg) } \\
\text { public removeMessage(String pt, Object msg) }\end{array}$ \\
\hline \hline
\end{tabular}

From the Table 5, we can see the differences between flattened component and composition (compound component) are:

1. If a component is a flattened component, there is no subcomponent instances in the constructor, nor connect() method invoked, which are numbered 1.a, 1.b, 1.c;

2. If a component is a flattened component, there is no connect pattern defined, which is numbered 2.

3. If a component is a flattened component, a Net instance must be declared and behavior model must be registered.

4. If a component is a flattened component, sendMessageFromPort() method must be overridden. This method has been declared in the template class.

5. If a component is a flattened component, run () method must be overridden. This method is from the thread implementation. 
It is worth to note that there is no definition for the requires port method in the Table 5. In ArchJava programming language, the implementation of requires port method is defined by the provides port method. The requires port method only provides an interface point that can be used to connect with the corresponding provides port method. Furthermore, each composition, component or connector in SAM will have one component class, thus each component class in ArchJava will have one object after instantiation. We can say that the number of architecture elements $N_{A S}$ and the number of component classes in ArchJava $N_{c o m}$ are equal, e.g., $N_{A S}=N_{c o m}$. Since all architecture elements are uniquely identified in SAM model, this result indicates that all component class instantiated once. In other words, there is only one object instantiated for each architecture elements in SAM.

\subsubsection{Behavior Model Translation}

Each composition, component or connector has a Petri net as its own behavior model in SAM specification. They connect to each other through ports. Finally these Petri nets form a large Petri net through ports connection.

The behavior model (Petri net) is translated to Java code. In our implementation, we define a template for both of the architecture and behavior model. Each concrete element in ArchJava/Java code can inherit from these templates. In other words, for each Petri net, we define some classes such as place, transition, arc, and some sorts etc.. Table 6. shows the mapping relation between each pair of elements in SAM behavior model and Java code.

\subsubsection{Property Specification}

Property specification in SAM model is expressed by temporal logic formula. Each associated property specifies a system behavior (structure) constraint for a component/connector. The atomic predicate in a property is a port holds some messages. During the verification, property specification is used to regulate what the system behavior would be. In the runtime checking code, this is implemented using AspectJ since it provides an online instrument and brings less performance penalty. The property implementation is discussed in the next section. 
Table 6: Mapping relation between Syntax of Petri net and Java

\begin{tabular}{|c|c|c|}
\hline Petri Nets & & Java \\
\hline \multirow[b]{2}{*}{$\begin{array}{l}\text { Interface } \\
\text { places }\end{array}$} & \multicolumn{2}{|c|}{ Java class (Place) } \\
\hline & $\begin{array}{l}\text { Fields: } \\
\text { String name; } \\
\text { String id; } \\
\text { Marking marking; } \\
\text { Net net; }\end{array}$ & $\begin{array}{l}\text { Methods: } \\
\text { //a set of get methods for fields } \\
\text { 1. getMarkings(); } \\
\text { 2. getTokenNo() } \\
\text { //a set of synchronous methods } \\
\text { 1. addTokens() } \\
\text { 2. removeTokens() } \\
\text { 3. addMarkings() } \\
\text { 4. removeTokens() }\end{array}$ \\
\hline internal places & \multicolumn{2}{|c|}{ Java Class (Place) } \\
\hline & \multicolumn{2}{|c|}{ Java Class (Transition) } \\
\hline Transition & $\begin{array}{l}\text { Fields: } \\
\text { Net net; } \\
\text { String name; } \\
\text { String id; } \\
\text { Guard guard; }\end{array}$ & $\begin{array}{l}\text { Methods: } \\
\text { //a set of get/set methods for fields } \\
\text { 1. IsEnabled }(/ / 3 \\
\text { 2. Fire }(\text { // fire this transition } \\
\text { 3. getEnabledAssignment }() \\
\text { 4. guardEvaluate }(/ / \text { call } \\
\text { guard.evaluate }()\end{array}$ \\
\hline & \multicolumn{2}{|c|}{ Java Class (Arcs) } \\
\hline $\operatorname{Arcs}$ & $\begin{array}{l}\text { Fields: } \\
\text { Net net; } \\
\text { String name; } \\
\text { String id; } \\
\text { Inscription insc; } \\
\text { Place place; } \\
\text { Transition transition; } \\
\text { boolean isFromPlace2Trans; }\end{array}$ & $\begin{array}{l}\text { Methods: } \\
\text { //a set of get/set methods for fields } \\
\text { 1. enabled }() / / 3 \\
\text { 2. getVariables }() \\
\text { 3. setInscription() }\end{array}$ \\
\hline
\end{tabular}




\subsubsection{Tool Support}

SAM parser, the tool we construct, consists of three parts: Petri net parser, which implements Petri nets in Java code semiautomatically (automatic if Petri nets meet some restrictions), SAM structure parser, which translates SAM structure such as components, connectors and ports to ArchJava [19] code, and property parser that constructs runtime verification code as aspects with the help from the logic engine. Different parts have different target programming languages. ArchJava is chosen as the target language in structure parser because it is an extension to Java that seamlessly unifies software architecture with implementation and uses a type system to ensure that the implementation conforms to architectural constraints. For more details about automatic generation of functionality code of SAM models, please refer to [71,72].

The logic engine, Maude [132] in our case, is used to synthesize efficient algorithms for checking linear temporal logic (LTL) formulae on a finite event trace. The algorithms implemented in Maude are described in [177]. Both algorithms for past time LTL formulae and future time LTL formulae are synchronous and there is no need to traverse events backwards. A middleware called logic server, which converts the generated algorithms to the target programming language, is introduced between the Property parser and the logic engine. A protocol between the Property parser and the logic server is defined to make the Property parser independent from the logic engine. The property parser synthesizes generated algorithms from the logic server for all properties defined in a component or connector, and constructs an aspect, in which runtime verification code is defined and weaved into the implementation of SAM models.

\subsection{Runtime Verification on SAM}

The purpose of runtime verification is to monitor, analyze and guide the execution of programs. Traditionally the correctness of a model is verified at design level, runtime verification provides additional correctness assurance at implementation level. 
Specific to our case, we need to monitor property specifications for each component/connector during model execution. These property specifications are described as temporal logic formulae. Following subsections describe the approach to generate runtime verification code for propositional and first order linear temporal logic formulae respectively.

\subsubsection{Propositional Linear Temporal Logic Formulae}

Algorithms proposed in [94] and [177] are adopted to evaluate future time LTL formulae and past time LTL formulae on finite execution traces respectively. Both of these two algorithms have been implemented in Maude, the chosen logic engine in our work.

The dynamic programming algorithm in [94] first enumerates sub-formulae of the input formula that satisfies the enumeration invariant: any formula has an enumeration number smaller than the numbers of all its sub-formulae. This algorithm is synchronous, i.e. it reports a violation of a formula as soon as the first event making the formula fail is captured. The algorithm in [177] is explored to evaluate future time LTL formulae. This algorithm is also synchronous and suitable for online monitoring. The notion of binary transition tree (BTT), a generalization of binary decision diagrams (BDD) [35], is introduced to minimize the calculation of next state, which is in general time consuming task. More specifically, this algorithm constructs from a LTL formula a binary transition tree finite state machine, which can be used to analyze execution traces without the need for a rewriting system. Therefore the algorithm can be implemented in traditional programming languages.

Fig. 14 shows the generated monitoring code by SAM parser for the composition ECompany in the online shopping case study (Chapter 8). The body of the switch statement is the binary transition tree finite state machine, generated by the algorithm in [177]. The case number of the switch statement is also the state number. Current state is recorded in a variable. This variable has different values to indicate different system states. State -1 indicates that the formula is satisfied by the current event trace, while state -2 indicates violation of the formula on the current event trace. 
Three different results can be returned from the execution of the monitoring code of a formula: true, false and neither true nor false called unsure. True means the formula is satisfied while false means the formula is violated. Unsure is an intermediate result, from which we cannot tell if the formula holds or fails. The intermediate result can be returned by the monitoring code of any type of formulae. If the monitoring code of a safety property returns unsure, it means the safety property does not fail during the previous execution. For a liveness property, unsure generally means it is not true during the previous execution. For the past time LTL formulae, the unsure result actually indicates this past time LTL formula does not fail during past.

\subsubsection{Synthesis}

In general, runtime verification consists of several parts: event script defining events related with formulae, compilation and instrumentation tools that insert codes into source or binary programs to capture events required in the event script, and runtime checker that evaluates properties or formulae over the captured event trace during the execution of the program. In our case, event script is implicit in SAM models due to its characteristics, i.e. each property describes a kind of relationship between messages in different ports. Therefore, runtime verification of SAM properties is and only is executed at the occurrence of sending and receiving a message to or from concerned ports. The SAM parser illustrated in section 1 is the tool that not only generates runtime checker automatically from SAM properties, but also acts as the instrumentation tool to weave runtime checker into SAM functionality code (also generated automatically by the SAM parser) through aspect, in which implied events is defined explicitly as pointcuts.

Aspect-Oriented Programming allows us to dynamically modify models or implementations to include code required for secondary requirements (in our case, it is runtime verification) without modifying the original code. In aspect-oriented programming, AspectJ [26] in our case, aspects wrap up pointcuts, advice, and inter-type declarations in a modular unit 
of crosscutting implementation. Fig. 14, which is a part of generated aspect for composition Consumer in Fig. 24, clearly shows the way to weave runtime verification code into functionality code through aspects.

\subsection{Summary}

Over the past decade, many architectural modeling notations and analysis techniques have emerged from the software architecture research community as a possible answer to many of the problems inherent in large, complex, distributed software systems. However, these architectural methods have not satisfiably addressed the relationship between the abstract architectural models and concrete system realizations [142]. On the other hand, a number of software interoperability technologies have emerged primarily from industry $[14,185,199]$ that provide solutions for combining implementation-level software components with powerful system building tools. However, these technologies rarely used the architectural models that typically precede the implementation and/or influence the key properties of the realized systems.

In this paper, we have discussed our attempt in bridging the gap between the model-based and implementation-based approaches as well as enhancing the quality of large scale, distributed systems. We have coupled an explicit architectural model with an implementation infrastructure through automatic code generation, accompanying the runtime monitoring and verification of the implementation. In doing so, we have developed a generic monitoring approach that is used to enhance the quality of distributed component software. The generality of the approach is achieved through using runtime monitoring technology, i.e. automatically generating functional code, synthesize and weave runtime verification code into the implementation through aspect oriented programming to implement software architecture description automatically. We believe that a more reliable system can be obtained by combining formal analysis techniques at design level and runtime verification at implementation level. We also extend the scope of runtime verification to a subset of first order LTL formulae. Although the subset is small, it is enough for most SAM properties 
such as response property involving quantifiers. Finally, both the functional code and monitoring code are generated modularly. This code generation method is efficient and scalable and the generation procedure is linear to the model size.

However, runtime verification technique has its own limitations which are discussed in the following two aspects. In the code generation from SAM model, it is hard to generate code from Petri net automatically [128]. Fully automatic code generation is only possible when certain restrictions are enforced [71]. In the runtime verification, not all properties can be verified during program execution. More specifically, safety properties can be verified on a finite or infinite event trace to detect the violation, while liveness properties can only be verified on a finite event trace to check if it holds. 


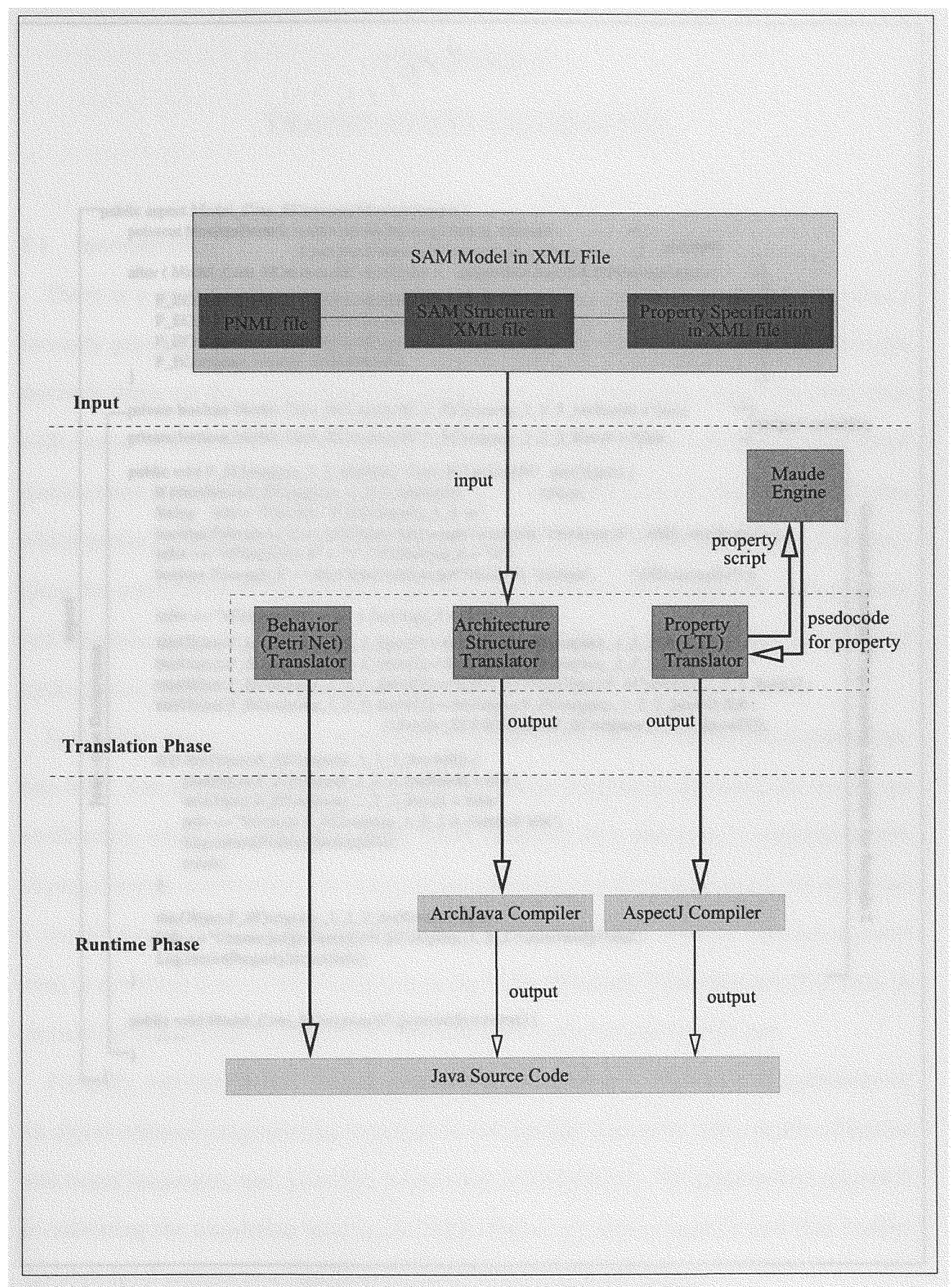

Figure 13: An Overview of Our Methodology 


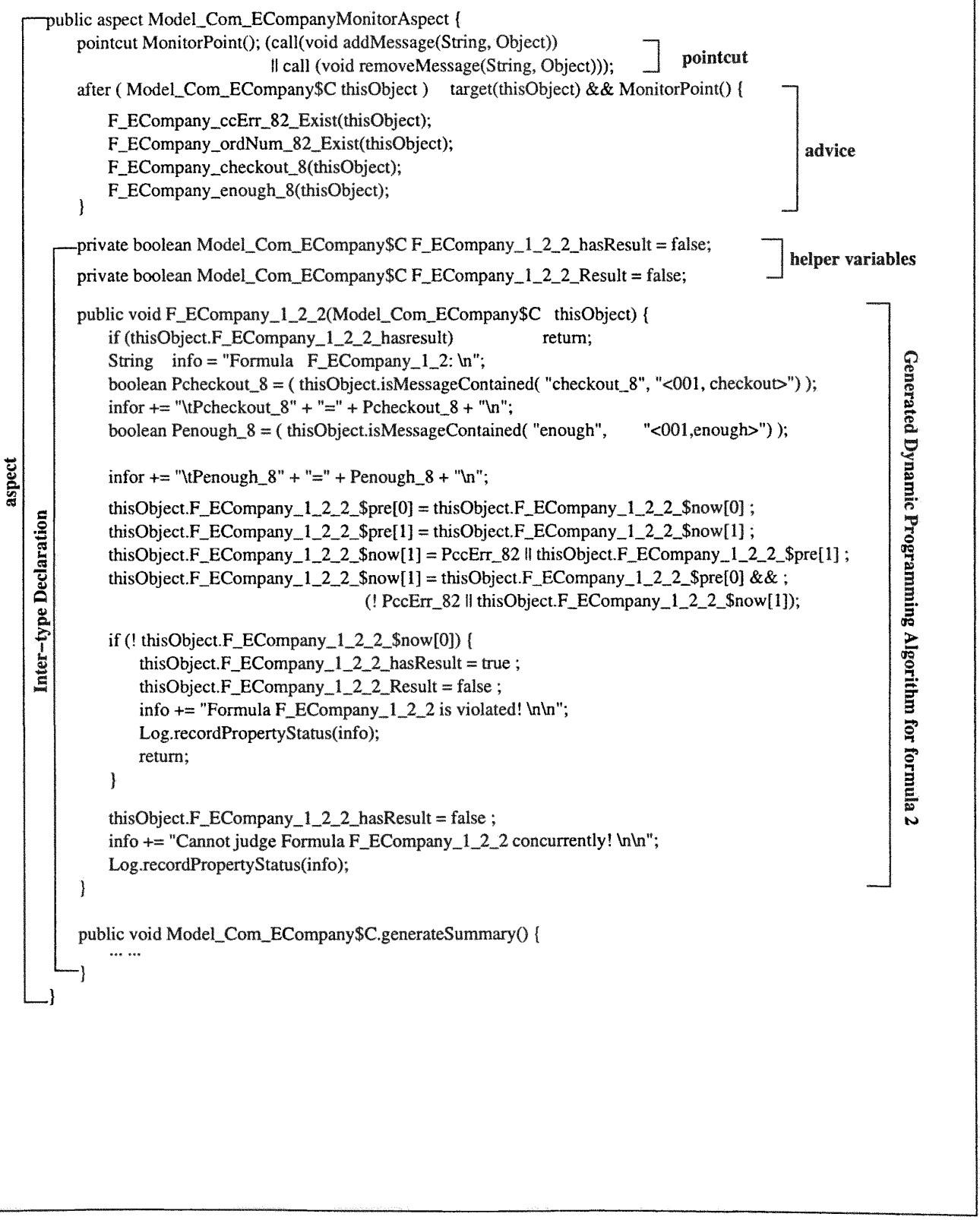

Figure 14: Generated Aspect for Composition ECompany 


\section{CHAPTER 7}

\section{TRANSLATION VALIDATION}

\subsection{Introduction}

There is a growing awareness, both in industry and academic, of the crucial role of formally proving the correctness of safety-critical portions of systems. Most verification methods focus on the verification of a design against requirements, and high-level code with respect to a given design specification. However, if one is to prove that the high-level specification is correctly implemented in low-level code, one needs to verify the translation which performs the conversion from high-level specification to the low-level code. Verifying the correctness of translation is challenging because of the complexity and syntactic and semantic diversity of the target architectures, as well as the sophisticated analysis and optimization algorithms used in the process of translation.

In this dissertation two translations have been developed in both design and implementation level. The translation from SAM model to Maude program is necessary for the design verification, while the translation from SAM model to Java construct is required for the implementation verification. To validate the translation in the design level, we construct a rewrite theory on a SAM behavior model from a stepwised relation between a proof term in rewriting theory and a transition firing in the Predicate Transition net (PrT net). A semantical consistency is proved between this rewrite theory and the $\operatorname{PrT}$ net.

Formally verifying a full-fledged general translation from a high-level specification to an object oriented programming language is not feasible, due to its size, variety of source and target languages, and, possibly, proprietary considerations. We proposed an approach to validating the translation used in the SAM Parser, a runtime analysis tool that verifies the high-level architecture specification SAM in the implementation. 


\subsubsection{Translation Correctness}

Generally speaking, software correctness is defined in terms of the desirable properties of a system. The correctness of software design must be correct in all observable aspects in correspondence with specification.

In the design level, we need to validate the translation from SAM model to the Maude program. The correctness of the Maude code depends on the specification of the architecture model being consistent with the specification of Maude in both structure and behavior. In other words, we need to show the semantical consistency between PrT net (behavior model of SAM) and rewriting logic (the principle of the Maude system and function modules). In section 7.3 we discuss how to prove the translation correctness from SAM model to Maude program.

In the runtime analysis, we verify the system properties of the translated code to show the correctness of the architecture model in the implementation. Thus the correctness of the translated architecture depends on two aspects: 1) implementation correctness - the correctness of code refers to whether the implementation satisfies the given property specification. 2) The correctness of translation define whether the behavior of an implementation agrees with that of specification. In the SAM Paser [71], we translated architecture model to a java construct (ArchJava/Java/AspectJ) for software architecture model (SAM) to automatically generate code and runtime checker. Runtime checker runs in parallel with architecture model code to verify the implementation correctness against system properties. In section 7.4 we discuss the correctness proof of translation from SAM to java construct in the runtime analysis supported by prototype tool SAM Parser.

The translation correctness refers to the agreement of the translated code with its specification, which is, in our case, a software architecture model. Therefore, describing the software translation $X$ as being correct is identical to stating that software translation $X$ meets all requirements imposed by the specifications [130]. In the formal method standing point of view, translation correctness is meant to be the combination of consistency and 
completeness. Translation consistency refers to the dynamic behavior in the source is preserved in the target. There are no internal contradictions, whereas translation completeness refers to the situations where each entity in the source is correctly reflected and represented in the target.

Definition 2 (Translation Correctness) Let $X$ be a translation that maps a specification $\mathcal{S} p$ to a target construct $\mathcal{P}, X: \mathcal{S} p \rightarrow \mathcal{P}$. The specification $\mathcal{S} p$ is a 3-tuple $\langle A, B, C\rangle$, where $A$ denotes the architecture structure, $B$ denotes the behavior of the specification, and $P$ denotes the constraints of a system. Let sem(spec) denotes the semantics of a specification spec $\in \mathcal{S} p$. The correctness of translation $X$ is defined by two following aspects:

1. Consistency: $\operatorname{sem}(B) \sim \operatorname{sem}(X(B))$, where $B \in \mathcal{S} p \wedge X(B) \in \mathcal{P}, \operatorname{sem}(C) \sim \operatorname{sem}(X(C))$, where $C \in \mathcal{S} p \wedge X(C) \in \mathcal{P}$;

2. Completeness: $\forall a \in A . \exists a^{\prime} \in \mathcal{P}$. such that $X(a)=a^{\prime}$, and $\forall b \in B . \exists b^{\prime} \in \mathcal{P}$. such that $X(b)=b^{\prime}$, and $\forall c \in A . \exists c^{\prime} \in \mathcal{P}$. such that $X(c)=c^{\prime}$.

\subsection{Related Work}

This work is an extension of the work in [71,72]. Work in [72] presents a method that efficiently translates software architecture (structure and behavior) model to ArchJava code. Work in [71] presented a methodology that translates structure, behavior and property of software architecture model and compile to Java code for the runtime analysis. Work in [76] extended the work in [72] to first order logic formula and applied to an embedded system example. None of these works $[71,72,76]$ validate the translations. Furthermore, the time complexity of the translation algorithm is analyzed, which is linear to the size of the model.

Currently many research works have been done on the translation validation for the optimizing compiler. The work in [157] covers some important aspects of transformation verification of semantics preservation. The work in [205] deals with the translation validation using runtime analysis, but there is not correctness notion. Another related work is [175] which proposed a comparable approach to translation validation, where an important contribution is the ability to handle pointers in the source program. However, the 
method proposed there assumes full instrumentation of the compiler, which is not assumed here or in [157]. The notion of correct translation that appears in [86] is similar to the one in the work [172]. However, [86] did not deal with optimizations of compiler.

More weakly related are the works reported in [156] and [158], which do not purport to establish full correctness of a translation but are only interested in certain "safety" properties. However, the techniques of program analysis described there are very relevant to the automatic generation of refinement mappings and auxiliary invariants. Rival [176] presents a methodology based on abstract-interpretation for certification of assembly code that uses the analysis of the source code and the debugging information.

The work in [70] presents a framework for describing global optimizations by rewrite rules with CTL formulae as side conditions, which allow for generation of correct optimizations, but not for verification of (possibly incorrect) optimizations. The correctness is established in an imperative languages without procedures. The work in [83] proposes a method for deploying optimizing code generation while correct translation between input program and code. They focus on code selection and instruction scheduling for SIMD machines.

Somewhat similar to our approach, in the treatment of software specification syntax transformation to the model checking programming language, is the work in [201]. There, static analysis is used to validate the syntax, semantics and property translations, on which software testing strategy is adopted to validate the translation. Preserving equivalence conditions was not checked in that work. Furthermore, this work focuses on static transformations that are manually applied in system design, while the focus of our work is automatical code generation using SAM Parser tool. The work in [120] describes a translation from textual transition system to Petri nets. They validated the translation by proof of the translated model are bisimularly equivalent with the source model based on the step by step comparison. Although it is a stepwise proof between translated model and the source model, The soundness and completeness cannot be established because it is based on the semi-formal explanation for the comparison by the natural language. 


\subsection{Validation of Translation from SAM to Maude}

First, we briefly survey a fragment of rewriting logic, that is relevant to our study. Then we transform Petri nets into the rewriting logic. We finally define the correspondence results between the standard SOS and the logic one, lifting it to the proved semantics.

\subsubsection{Rewriting Theories and Deduction}

The mapping of a Petri net model into a rewriting theory requires fairly sophisticated algebraic techniques. As a first step, we recall the set-theoretical construction of the term algebra for many-sorted signatures [84]. In the following we first recall the rewriting logic and membership equational logic.

\section{Basic Definition}

An equational logic is a pair $(\Sigma, E)$, where $\Sigma$ is a set of operations, also called its "syntax", and $E$ is a set of equations of the form $\forall X . t=t^{\prime}$ constraining the syntax, where $X$ is some set of variables and $t, t^{\prime}$ are well-formed terms over variable set $X$ and operations in $\Sigma$. Equational logics can be many-sorted (operations in $\Sigma$ have arguments of specific sorts), or even order-sorted (sorts with a partial order on them). Equations can be conditional or unconditional. Conditions is a typically finite set of pairs $u=u^{\prime}$ over set of variables $X$.

Term rewriting is an approach related to the equational logic $(\Sigma, E)$ in which equations are oriented from left to right, with the form $\forall X . p \rightarrow q$ if $\bigwedge_{i} u_{i} \rightarrow u_{i}^{\prime}$, and called rewrite rules. A rewrite rule can be applied to a term $t$ at any position where $p$ is matched. A pair $(\Sigma, R)$, where $R$ is a set of rewrite rules, is called a rewrite system.

Rewriting logic [144] is a logic for concurrency. A rewrite theory is a tuple $(\Sigma, E, L, R)$, where $(\Sigma, E)$ is an equational logic, $L$ is set of labels, and $R$ is a set of rewrite rules. Rewriting logic therefore extend equational logic with rewrite rules, allowing one to derive both equations and rewrites. Deduction remains the same for equations, but the symmetry rule is dropped for rewrite rules. Rewriting logic is a framework for true concurrency [145]: the locality of rules allows multiple rules to apply at the same time provided they don't modify the shared part. A formal definition of a labeled rewrite theory is given in the following. 
Definition 3 An many-sorted labeled rewrite theory $\mathcal{R}$ is a 4-tuple $<\Sigma, E, L, R>$ where $\Sigma$ is an many-sorted signature, $E$ is a set of $\Sigma$-equations, $L$ is the set of labels, and $R \subseteq$ $L \times T_{\Sigma, E}(X)^{2}$ is the set of labeled rewrite rules.

We often write $l:[s] \rightarrow[t]$ if $[\vec{u}] \rightarrow[\vec{v}]$ for $l:[s] \rightarrow[t]$ if $\left[u_{1}\right] \rightarrow\left[v_{1}\right] \wedge \ldots \wedge\left[u_{k}\right] \rightarrow\left[v_{k}\right]$.

Rewrite rules in $\mathcal{R}$ may be understood as the basic rewriting steps of a theory, the building blocks of the actual rewrite relation. More complex deductions can be obtained by a finite number of applications of inference rules. We introduce a suitable signature for building an algebra of labels, each element of the term algebra encoding a justification of a rewrite. Out of all possible different ways to introduce such a signature, we follow the lines of [49].

Definition 4 (rewriting step) Let $\mathcal{R}=<\Sigma, E, L, R>$ be a rewrite theory, let $\Omega$ be the signature containing all the labels $r$ as suitable operators, with the corresponding arity and sort given by the variables in $R(r)$

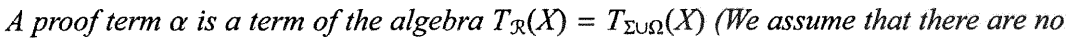
clashes of names between the sets of operators).

A rewriting step is a triple $<\alpha,[s],[t]>$ (usually written as $\alpha:[s] \rightarrow[t]$ ) where $\alpha$ is a proof term and $[s],[t] \in T_{\Sigma, E}(X)$.

As argued in [144], "a rewrite theory is just a static description of 'what a system can do'; the behavior of the theory is instead given by the rewrite relation induced by the set of rules of deduction". Given a set of rewriting rules, we can derive a series of rewriting theorems about the system. This procedure is called entailment and defined in the next definition.

Definition 5 (Entailment) Let $\mathcal{R}=<\Sigma, E, L, R>$ be a rewrite theory. We say that $\mathcal{R}$ entails the rewriting step $\alpha:[s] \rightarrow[t]$, written as $\mathcal{R} \vdash \alpha:[s] \rightarrow[t]$, if and only if it can be obtained by a finite number of applications of the inference rules in Table 7. 


\begin{tabular}{|ll|}
\hline (reflexivity) & $\frac{[t] \in T_{\Sigma, E}(X)}{t:[t] \rightarrow[t]}$ \\
(transitivity) & $\frac{\alpha:[s] \rightarrow[u], \beta:[u] \rightarrow[t]}{\alpha ; \beta:[s] \rightarrow[t]}$ \\
(congruence) & $\frac{f \in \Sigma_{n}, \alpha_{i}:\left[s_{i}\right] \rightarrow\left[t_{i}\right]}{f\left(\alpha_{1}, \ldots, \alpha_{n}\right):\left[f\left(s_{1}, \ldots, s_{n}\right)\right] \rightarrow\left[f\left(t_{1}, \ldots, t_{n}\right)\right]}$ \\
(replacement) & $\frac{\alpha_{i}:\left[w_{i}\right] \rightarrow\left[z_{i}\right], R+r:[s] \rightarrow[t]}{r\left(\alpha_{1}, \ldots, \alpha_{n}, \alpha\right):[s(\vec{w} / \vec{x})] \rightarrow[t(\vec{z} / \vec{x})]}$ \\
(equality) & $\frac{E \vdash s=u, \alpha^{\prime}: u \rightarrow u^{\prime}, E+u^{\prime}=t}{\alpha: s \rightarrow t}$ \\
(composition) & $\frac{\alpha:[s] \rightarrow[u], \beta:[u] \rightarrow[t]}{\alpha ; \beta:[s] \rightarrow[t]}$ \\
(associativity) & $\frac{-}{\alpha ;(\beta ; \gamma) \equiv(\alpha ; \beta) ; \gamma}$ \\
(distributivity) & $\frac{f \in \Sigma}{f\left(\alpha_{1} ; \beta_{1}, \ldots, \alpha_{n} ; \beta_{n}\right) \equiv f\left(\alpha_{1}, \ldots, \alpha_{n}\right) ; f\left(\beta_{1}, \ldots, \beta_{n}\right)}$ \\
\hline
\end{tabular}

\section{Inference Rules}

The deduction system in Table 7 was introduced in [144]. Some rules are very easily to be understood, such as reflexivity, transitivity, equality, etc. Here we only explain one of them, replacement rule, as an example. Replacement rule implies that the transition relation is stable, that is, it is closed under substitution. Moreover, the associated rewriting step describes the simultaneous execution of nested rewrites: Two subterms matching the left-hand sides of two rules can be rewritten in parallel even if their roots are not disjoint, i.t., if one is above the other, provided that they do not overlap.

Indeed, in [144] Jose Meseguer establishes a correspondence between deductions in rewriting logic and concurrent computations, defining a model for a rewrite theory as a system whose states are $E$-equivalence classes of $\Sigma$-terms, and whose transitions are equivalence classes of terms representing proofs in rewriting deduction, that is, concurrent rewrites using the rules in $R$. 
The first five rules can be visualized in the Figure. 15 [147].

Reflexivity

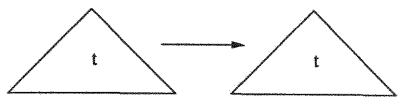

Equality

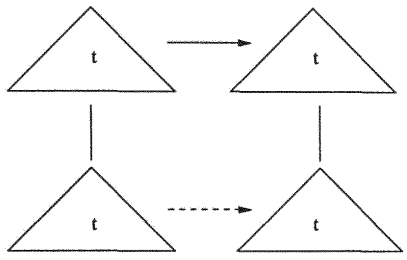

Transitivity
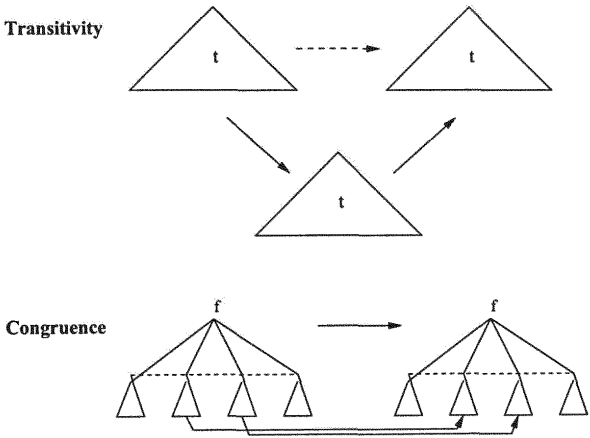

Replacement

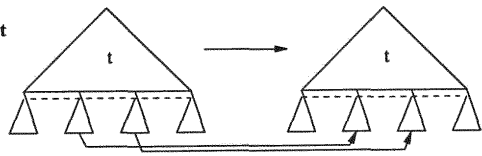

Figure 15: Visualization of 5 Inference Rules

\subsubsection{A theory for Petri nets}

Given a PrT net $N=(N e t, S$ pec, Ins $)$ [96], where

- Net $=(P, T, F)$, where $P, T$ are finite set of places and transitions, and $P \cap T=\Phi$, $F=P \times T \cup T \times P$ is a set of flow relation. 
- $S$ pec $=(S, O P, E q)$, signature $\Sigma=(S, O P)$ where $S$ is a set of sorts, and $O P$ is a set of operations on sorts $s \in S, E q$ is a set of $\Sigma$-equations.

- Ins $=\left(\phi, L, R, M_{0}\right)$, where $\phi: P \rightarrow \mathcal{P}(S)$ is a sort assignment function, $L: F \rightarrow$ Label $_{S}(X)$ is a sort-respecting labeling function of $N$ (where $X$ is the set of variables), $R: T \rightarrow \operatorname{Term}(X)$ is a constraint mapping from each transition $t \in T$ to a boolean logic formula $\operatorname{Term}(X)$, and $M_{0}: P \rightarrow M C O N_{S}$ is a sort-respecting initial marking of $N$.

we define a rewriting theory $\mathcal{R}^{\operatorname{Pr} T}=\left(\Sigma_{\mathcal{R}}, E_{\mathcal{R}}, L_{\mathcal{R}}, R_{\mathcal{R}}\right)$ for each ingredient of PrT nets as follows. If there is no confusion in the context, we refer $\mathcal{R}$ simply as $\mathcal{R}^{P r T}$.

- Signature $\left(\Sigma_{\mathcal{R}}, S_{\mathcal{R}}\right)$ with $S \mathcal{R}$ is a set of sorts, and $S \mathcal{R}=S_{P r T}$, where $S_{P r T}$ denotes the set of sorts in PrT nets, and $\Sigma_{\mathcal{R}}=\Sigma_{P r T}$, where $\Sigma_{P r T}$ denotes signature in PrT specification. We define a sort named marking in the Maude implementation, thus we have marking $\in S_{\mathcal{R}}$.

- $E_{\mathcal{R}}=E q$.

- $L_{\mathcal{R}}$ is set of labels, which is defined by a function such that $L_{\mathcal{R}}: P \cup T \rightarrow f_{\mathcal{\Sigma}} \cup R_{\mathcal{R}}$, where $f_{\Sigma}$ denotes the operators on the signature $\Sigma$, if no confusion, we simply refer $f$ as $f_{\Sigma}$.

- $\forall p \in P . L_{\mathcal{R}}(p)=f_{\Sigma}$, where $f$ is defined as a mapping from sorts of $p$ to the sort marking, i.e., $f: s \rightarrow$ marking, where $s$ is the sort of the place $p$.

- $\forall t \in T \cdot L_{\mathcal{R}}(t): \alpha$, where $\alpha$ is a rewriting step defined as $\alpha: \operatorname{pre}(t) \rightarrow \operatorname{post}(t)$ if $R_{P r T}(t)$, where $R^{P r T}$ is the constraints defined in the PrT net, which is a mapping function from transition $T$ to the boolean terms $\operatorname{Term}_{O p, b o o l}$. pre $(t)$ and post $(t)$ is the preset and the postset of transition $t$, respectively, and they are $\Sigma$-terms in $\mathcal{R}_{P r T}$.

- $\forall p \in P . M_{0}(p)=L(p)(C O N s)$, where CONs is a set of constants. 
- $S$ pec $=(S, O P, E q)$, signature $\Sigma=(S, O P)$ where $S$ is a set of sorts, and $O P$ is a set of operations on sorts $s \in S, E q$ is a set of $\Sigma$-equations.

- Ins $=\left(\phi, L, R, M_{0}\right)$, where $\phi: P \rightarrow \mathcal{P}(S)$ is a sort assignment function, $L: F \rightarrow$ Label $_{S}(X)$ is a sort-respecting labeling function of $N$ (where $X$ is the set of variables), $R: T \rightarrow \operatorname{Term}(X)$ is a constraint mapping from each transition $t \in T$ to a boolean logic formula $\operatorname{Term}(X)$, and $M_{0}: P \rightarrow \operatorname{MCON}_{S}$ is a sort-respecting initial marking of $N$.

we define a rewriting theory $\mathcal{R}^{P r T}=\left(\Sigma_{\mathcal{R}}, E_{\mathcal{R}}, L_{\mathcal{R}}, R_{\mathcal{R}}\right)$ for each ingredient of PrT nets as follows. If there is no confusion in the context, we refer $\mathcal{R}$ simply as $\mathcal{R}^{\operatorname{Pr} T}$.

- Signature $\left(\Sigma_{\mathcal{R}}, S_{\mathcal{R}}\right)$ with $S \mathcal{R}$ is a set of sorts, and $S \mathcal{R}=S_{P r T}$, where $S_{P r T}$ denotes the set of sorts in PrT nets, and $\Sigma_{\mathcal{R}}=\Sigma_{P r T}$, where $\Sigma_{P r T}$ denotes signature in PrT specification. We define a sort named marking in the Maude implementation, thus we have marking $\in S_{\mathcal{R}}$.

- $E_{\mathcal{R}}=E q$.

- $L_{\mathcal{R}}$ is set of labels, which is defined by a function such that $L_{\mathcal{R}}: P \cup T \rightarrow f_{\Sigma} \cup R_{\mathcal{R}}$, where $f_{\Sigma}$ denotes the operators on the signature $\Sigma$, if no confusion, we simply refer $f$ as $f_{\Sigma}$.

- $\forall p \in P . L_{\mathcal{R}}(p)=f_{\Sigma}$, where $f$ is defined as a mapping from sorts of $p$ to the sort marking, i.e., $f: s \rightarrow$ marking, where $s$ is the sort of the place $p$.

- $\forall t \in T . L_{\mathcal{R}}(t): \alpha$, where $\alpha$ is a rewriting step defined as $\alpha: \operatorname{pre}(t) \rightarrow \operatorname{post}(t)$ if $R_{P r T}(t)$, where $R^{P r T}$ is the constraints defined in the PrT net, which is a mapping function from transition $T$ to the boolean terms $\operatorname{Term}_{O p, b o o l} . \operatorname{pre}(t)$ and $\operatorname{post}(t)$ is the preset and the postset of transition $t$, respectively, and they are $\Sigma$-terms in $\mathcal{R}_{\text {PrT }}$.

- $\forall p \in P . M_{0}(p)=L(p)(C O N s)$, where CONs is a set of constants. 
- $R_{\mathcal{R}}$ is defined by the firing rule for each transition $t \in T . \forall l \in R$,

- if $l$ is unconditional rule with the form $l:[p] \rightarrow[q]$, then we have $p, q \in S$ with $\Sigma$-algebra, with $p=\operatorname{pre}(t)$ and $q=\operatorname{post}(t)$.

- if $l$ is conditional rule with the form $l:[p] \rightarrow[q]$ if $\wedge_{i} C_{i}$ where $C_{i} \in$ Term $_{O p, b o o l}(X)$ and $\forall v_{i} \in C_{i} \cdot v_{i} \in X, p$ and $q$ are pre $(t)$ and postt of $t$ respectively., $\bigwedge_{i} C_{i}=g_{i, t}$, where $g_{i, t}$ is constraint for transition $t$ and $g_{i, t} \in R_{P r T}$ (a constraint function defined in the PrT).

It is worth to note that $E_{\mathcal{R}}$ can be empty.

\subsubsection{An interleaving correspondence}

We state a claim that relates the semantics of PrT nets based on the rewriting theory $\mathcal{R}^{\text {PrT }}$ with the dynamics stated in the Chapter 3. To this end, we need to define and characterize a few classes of proof terms.

Definition 6 Let $\alpha$ be a closed proof term over the rewriting theory $\mathcal{R}_{\text {PrT }}$. Then we have $\alpha$ is

- is initial, if it is element of sort $S_{P r T}$.

- is enabled, if it is initial and contains occurrence of an operator in $O P$ and $E \neq \Phi$.

- is one step, if it is enabled and contains no occurrence of $M(p)$ with $p \in P$ and no occurrence of composition operator :

- is fired, if it is one step.

- is many steps, if $\alpha=\alpha_{1} ; \ldots ; \alpha_{n}$ with $1 \leq n \leq \Sigma$ and each $\alpha_{i}$ is one step.

- is rewriting step, if it is many steps and all the one-step rewriting step is enabled.

In the following, we define the relation between an occurrence for a transition $t \in T$ of a PrT net and a rewriting step $\alpha$. 
Definition 7 An occurrence for a transition $t \in T$ of a PrT net $N$ is a rewriting step $\alpha_{t}$ : $[p] \rightarrow[q]\left(\right.$ if $\left.\wedge_{i} C_{i}\right)$ with the following conditions:

- a transition $t$ is initial, if $R(t) \in \operatorname{Term}(X)$ and $\alpha_{t}$ is initial.

- a transition $t$ is enabled, if $t$ is initial and $\alpha_{t}$ is enabled.

- a transition $t$ is fired once under marking $M_{1}$ and reach marking $M_{2}$, if $\alpha_{t}$ is one step.

This characterization is needed to prove the correspondence between PrT nets and rewriting theory $\mathcal{R}_{P r T}$ : given a PrT net $N$, there is a one-to-one correspondence between the computation sequence of $\operatorname{Pr} T$ net $N$ and the rewriting theory $\mathcal{R}_{P r T}$.

Proposition 1 (One-step Correspondence) Let $t \in T$ be a transition in $\operatorname{Pr} T$ net $N, M_{1}$ and $M_{2}$ be two markings before and after $t$ is fired, then a one step computation sequence for PrT net $N$ is $M_{1} t M_{2}$.

If $\mathcal{R}_{P r T}$ entails an initial, one-step rewriting step $\alpha:($ pre $\left.(t))(s) \rightarrow(p o s t(t))(s)\right)$, then there is a computation sequence $M_{1} t M_{2}$ with $(\operatorname{pre}(t))(s) \in M_{1}$ and $(\operatorname{post}(t))(s) \in M_{2}$ defined for PrT nets.

Proof.

The proof has two steps on the definition of rewriting step.

- For any transition $t \in T, \exists M$ such that $(\operatorname{pre}(t))(s) \in M_{1}$, and

- for any transition $t \in T, \exists M$ such that $(\operatorname{post}(t))(s) \in M_{1}$.

Based on the definition 4, we can conclude the above proposition. endProof.

We already observed in previous definition that each place defines an $\Sigma$-signature on the set of sorts and the fixed sort marking as the operation/function with arguments. The markings are connected by transition firing defined by the Petri net semantics [96]. Thus we can conclude this section by the correspondence result between Petri net computational sequence semantics and the rewriting theory semantics. 
Corollary 1 Let $M_{i}$ and $M_{j}$ be two markings of a PrT net $N$. Then a computation sequence $M_{i} \ldots M_{j}$ entails a transition $M_{i}$ to $M_{j}$ iff $\mathcal{R}^{P r T}$ entails a rewriting step $\alpha: M_{i}(P) \rightarrow M_{j}(P)$.

Finally, next result lifts the correspondence to computations.

Proposition 2 (Computational Correspondence) Let $M_{c 0}$ and $M_{c j}$ be two markings of a $\operatorname{PrT}$ net $N$. Then there exists a proved computation $\sigma=M_{c 0} t_{c 0} \ldots t_{c j-1} M_{c j}$ with source $M_{c 0}$ and target $M_{c j}$ iff $\mathcal{R}_{P_{r T}}$ entails an initial one step sequential rewriting step $\alpha=\alpha_{1} ; \ldots ; \alpha_{m}$ with $\alpha_{i}: L\left(\operatorname{pre}\left(t_{k}\right)\right)(s) \rightarrow L\left(\operatorname{post}\left(t_{k}\right)\right)(s)$ where $i \leq k \leq j$.

\section{Proof.}

This can be proved inductively on the computation sequence.

Base case: $k=c 0$ we have the computation sequence $M_{c 0} t_{c 0} M_{c 1}$, from Proposition for the One-step Correspondence, we have $\alpha_{c 0}: L\left(\operatorname{pre}\left(t_{c 0}\right)\right)(s) \rightarrow L\left(\operatorname{post}\left(t_{c 0}\right)\right)(s)$.

Hypothesis Assumption: Suppose it is true for $k=n$ where $i \leq n \leq j$ the proposition for computational correspondence holds. Then we have $\alpha=\alpha_{1} ; \ldots ; \alpha_{m}$ with $\alpha_{i}:\left(\operatorname{pre}\left(t_{k}\right)\right)(s) \rightarrow\left(\operatorname{post}\left(t_{k}\right)\right)(s)$ holds. Because $\mathcal{R}_{P r T}$ entails an initial one step sequential rewriting step $\alpha=\alpha_{1} ; \ldots ; \alpha_{m}$, there exists one rewriting step $\alpha_{I}: \alpha_{1} ; \ldots ; \alpha_{i}$ where $i \leq k \leq j$ and $\alpha_{i}:\left(\operatorname{pre}\left(t_{k}\right)\right)(s) \rightarrow\left(\operatorname{post}\left(t_{k}\right)\right)(s)$. We need to show for $k=n+1$ it still holds.

If $k=n+1$, we need to consider two situations:

- (1) one is that $\operatorname{post}\left(t_{n}\right)=\operatorname{pre}\left(t_{n+1}\right)$;

- (2) $\operatorname{post}\left(t_{n}\right)$ is not $\operatorname{pre}\left(t_{n+1}\right)$. Because we use interleaving semantics for the PrT nets, and in the rewriting theory, each rewriting rule can be executed concurrently, $t_{n}$ and $t_{n+1}$ may be in a causal relation.

Based on the one-step correspondence, for each transition firing, we can have a rewriting step, so for $t_{n+1}$ under some marking $M_{n}$, we have $\left.\alpha_{c n+1}:\left(\operatorname{pre}\left(t_{c n+1}\right)\right)(s)\right) \rightarrow\left(\operatorname{post}\left(t_{c n+1}\right)\right)(s)$.

For case (1), under the marking $M_{n}$, based on the hypothesis assumption, we know that $\left(\operatorname{post}\left(t_{n}\right)\right)=\left(\operatorname{pre}\left(t_{n+1}\right)\right)$. From transition rule, the proposition holds. 
For case (2), there must be some transition $t_{h}$ fired and make the proposition holds, i.e., let $t_{h}$ be the transition in the hypothesis assumption, that is $t_{h}=t_{n}$. Similarly, the proposition holds.

endProof.

\subsection{Validation of Translation from SAM to Java Construct}

As discussed in Chapter 6, the translation from SAM model to java construct includes three parts - architecture structure translation, behavior model translation, and property translation. The target programming languages for the three parts are ArchJava, Java, and AspectJ. The structure of the translation can be shown in the Figure 16.

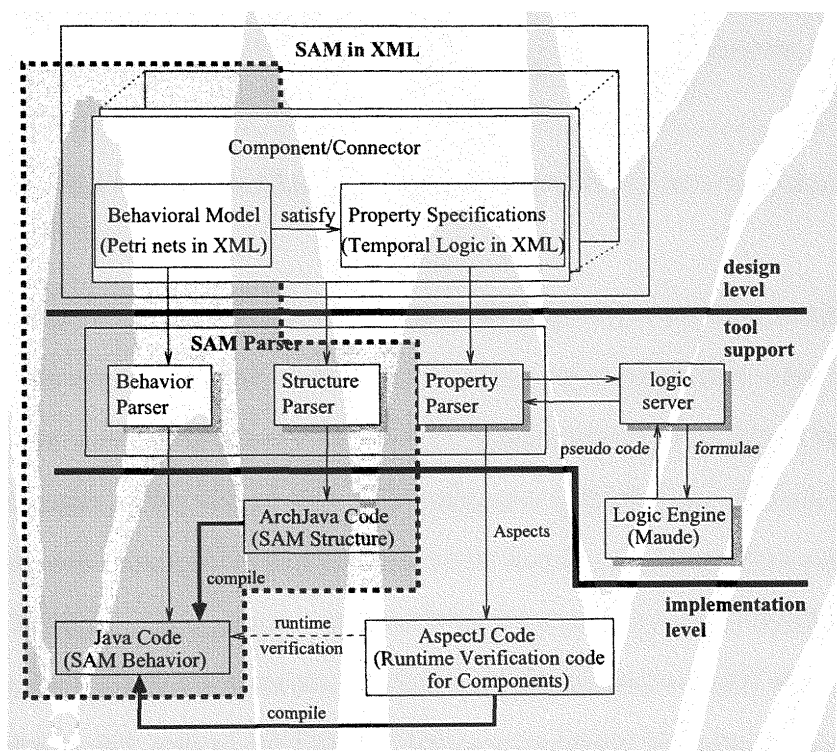

Figure 16: An Architecture of Translation

Both ArchJava and AspectJ code are compiled to java code, they are synthesized with java code during execution. The translated properties are used to generate the runtime monitor to validate the system model. In this section, we only consider how to validate the architecture structure and behavior model translation. These parts are highlighted in 
the Figure 16. As discussed in the introduction, the "validation" here means to show the translation correctness of the java construct. Since ArchJava has be precompiled into java code, so here we consider only the correctness proof of java implementation against the SAM model. The proof methodology is shown in the Figure 17.

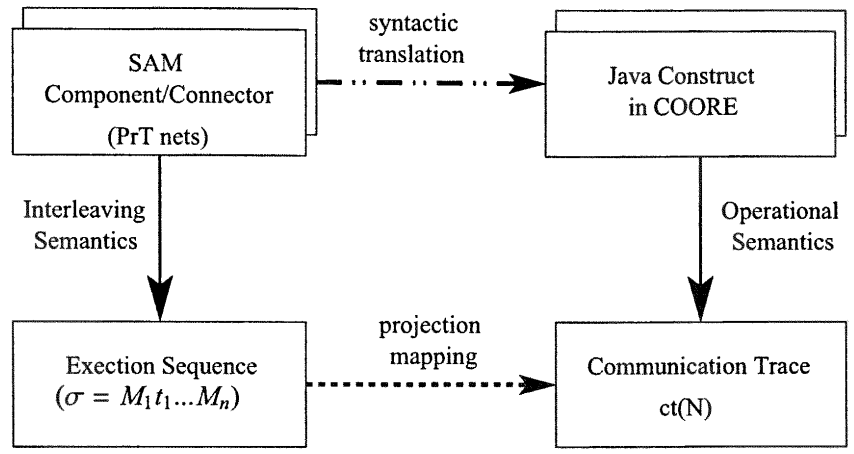

Figure 17: The Proof Methodology of Translation to Java Construct

The proof can be captured in the following steps (Fig. 17). First, some restrictions are enforced on the sorts and variables for the PrT nets. These restrictions make the PrT net fits for the java syntax, but do not have effect on the expressive power of the PrT nets, which is discussed in the next section. Secondly, we simplified and represented the java construct for each component/connector of SAM model into a sequential java assertion language (COORE). This is simply represented by the arrow "syntactic translation" in Fig. 17. Thirdly, we construct a syntactic relation from the behavior model of SAM component/connector (a PrT net) to the COORE-like java assertion language. The operational semantics for the java assertion langauge is given. This is simply represented by the arrow "operational semantics" in Fig. 17. Moreover, we define the communication trace for the java assertion language based on its semantics. Finally, a mapping relation is established from the projection on the interleaving semantics of PrT net and projection on the communication trace. This is simply represented by the arrow labeled as "projection mapping" in Fig. 17. 


\subsubsection{Interleaving Semantics of Restricted PrT nets}

In this section, we first give some restrictions for the PrT nets. One of the reasons is with these restrictions, we can automatically generate the behavior code for the SAM model. These restrictions do not have effect on the expressive power of PrT nets. After that, we give the sequential semantics of the restricted PrT nets and the firing rule for each enabled and fired transition. The transition based firing rule are useful for the further analysis of the semantics consistency.

The following restriction if imposed on the PrT nets:

1. The sorts of PrT nets are either Java primitive types (denoted by $D_{\text {java }}$ ) such as int, boolean etc., or defined as a Java class (denoted by $D_{\text {java-def }}$ ). A product type $P_{D}$ can be defined by either primitive type or user defined type or mixed, can be implemented as a user defined class. Thus we have follows:

$$
D::=D_{\text {java }}\left|D_{\text {java-def }}\right| D_{\text {prod }} \text { where } D_{\text {prod }}=D(\times D)^{*}
$$

2. The variables occurred in the label of an incoming arc of a transition have the same type as the token sort of the incoming place.

3. The variables occurred in the label of an outgoing arc of a transition is defined in the label of an incoming arc of the same transition. In other words, only the label of an incoming arc can define new variables.

4. The trap places only appear in the places that have Set sort.

5. Each Set sort is assumed to have finite number of elements. Thus the substitutions for each variable in the guard are finite since we model a closed system.

All the following subsections of this section refers restricted version of PrT nets based on this definition. If there is no confusion we would use PrT nets instead of restricted version of PrT nets during this section.

An occurrence of a restricted PrT net is defined by all substitutions of the variables in the labels of the arc and the Boolean expressions in the guard and this set of substitutions 
make the evaluation of the guard true. For the first order formula, it can be infinite sets of substitutions for a true evaluation of a guard. Thus for each restricted PrT net, it is possible to have infinite number of process nets.

A semantics is a mapping that assigns to every element of a syntactic domain a meaning or interpretation, i.e. an element of a semantic domain. Usually the semantics of Petri nets is given by means of sequence of states - markings. Different markings indicate the transition firing rules. This approach is known as an interleaving approach and it is based on a single observer model in which the occurrence of events (transitions) is serialized even for independent processes.

We can define the interleaving semantics using the sequence of markings with the occurrence of corresponding transitions for each set of substitutions.

Definition 8 (Interleaving Semantics of PrT nets) The sequence $\sigma=M_{0} t_{0} M_{1} t_{1} \ldots t_{n-1} M_{n}$ with $n \geq 0$ is called a finite interleaving execution starting with $M_{0}$ iff $\forall i \in N a$ and $0 \leq i \leq n$ and $M_{i-1} \rightarrow t_{i-1} M_{i}$, where $M_{i}: P \rightarrow \mathcal{P}(S t)$.

where $S t$ represents the set of sorts. The interleaving semantics of a PrT net is defined on a sequence of fired transitions with markings. The state sequence $\sigma$ of a PrT net can be either finite or infinite, depending on the system we modeled. Even though we restricted the tokens in each Set to be finite, and the output tokens are produced to output ports finally, the local infinite loop structure with some specific guard conditions can also enable and fire a transition infinitely and thus generates infinite state of sequence. A definition of interleaving semantics for the S/T systems was given in the Best's book [28]. The markings for the $\mathrm{S} / \mathrm{T}$ systems [28] represents the data without sorts information and the transitions do not have guard conditions.

It is well known that Petri net is a model having true concurrency as well as interleaving semantics. A common model for representing concurrent software is the interleaving model, in which all of the events in a single execution are arranged in a linear order called 
an interleaving sequence. Concurrently executed events appear arbitrarily ordered with respect to one another. Most logics for specifying properties of concurrent systems cannot distinguish between interleaving sequences in which two independent events are executed in different orders. Because of this, all possible interleavings of such events are normally considered. Due to this reason, we can say that there is no information loss in the interleaving model. In our work we choose the interleaving semantics because it has no information loss and is wildly applied in practice.

To show the Java construct is correctly translated from SAM model, we use communication history or trace defined in CSP [149] model to describe the execution path. First, we simplify and reduce the java program for each component/connentor in SAM to a sequential program. This program is represented by an assertion language with COORE-like syntax [170]. Then, we define the elements of a communication trace in this assertion language based on its operational semantics. After that, a mapping relation is established between the COORE-like syntax representation and the java construct to identify the correspondence between each element in the communication trace of an execution path and the state in a PrT execution. Finally, we show that the correspondence between the communication trace and the state sequence based on the mapping relation.

In the interleaving semantics of PrT nets, we examine each individual transition instead of set of transitions during the execution. As markings represent a global state of the system, during each transition firing, the state changing only effects the places that related to the fired transition, i.e., preset and postset of the transition. Thus preset and postset of a transition with the transition form a sequence that provides a local view for the system execution.

We represent a firing for a transition $t$ under marking $M$ with binding $\alpha$ (occurrence mode) as follows:

Let $\left\{p_{r 1}, \ldots, p_{r m}\right\}$ be the preset of the transition $t,\left\{p_{o 1}, \ldots, p_{o n}\right\}$ be the postset of the transition $t$, for one execution path $i$ of $t, \alpha$ be a binding of a set of variables related to $t$. For some execution path $i$ of $t$, we have the firing for a transition $t$ as follows: 


$$
\left\{p_{r 1}(\alpha, t), \ldots, p_{r m}(\alpha, t)\right\} t\left\{p_{o 1}(\alpha, t), \ldots, p_{o n}(\alpha, t)\right\}
$$

The firing rule for each individual transition provides a local view for each place as well as tokens in that place with regard to the transition. This definition provides a possible local reasoning for the system on the interleaving semantics.

For instance, in the model of online shopping example (the User component of the onlineshopping example Chapter 8). Initial markings are given as in the figure in Chapter 8 . Suppose after some execution, there is a token in the input port uinfoReq-5, then transition $t_{7}$ is enabled. Let the current marking be $M_{1}$, after $t_{7}$ is fired, the marking is $M_{2}$. The preset of the transition $t_{7}$ is in the marking $M_{1}$, and the postset of the transition is in the marking $M_{2}$. Let take a look at the marking of the preset and postset of the transition, we have the sequence:

$\left\{U_{\operatorname{serID}}\left(\right.\right.$ uid $\left._{i}\right), u \operatorname{Info}\left(\right.$ uid $\left._{i}\right), u \operatorname{InfoReq}_{5}\left(\right.$ uid $\left.\left._{i}, \operatorname{Req}\right)\right\} t_{7}$

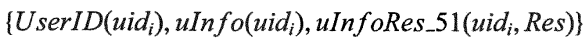

Whenever the transition $t_{7}$ is fired, the sequence

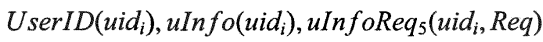

would be in the marking $M_{1}$, and the sequence

UserID $\left(\right.$ uid $\left._{i}\right)$, Info( $\left(\right.$ uid $\left._{i}\right)$, uInfoRes_51(uid, , Res $)$

would be in the marking $M_{2}$.

It is worth to note that every time the transition $t_{7}$ is fired, and if the order of places is fixed, the above firing pattern can be observed except for the tokens in some place might be different.

\subsubsection{Sequential Semantics of Simplified Java Construct}

In this subsection, we first overview the features of the SAM and the java construct generated from SAM Parser. Then we use a COORE-like syntax assertion language to represent the java construct. The operational semantics for this COORE-like assertion language is listed. Finally, the sequential semantics of the simplified java construct is presented. 


\section{Simplification of Java Construct}

To fully automatically generate code of PrT nets from SAM Parser, several restrictions has been made on the SAM behavior model [71]. The algorithm for the execution of the generated code is highly dependent on the algorithm chosen for the transition firing. Generally used algorithm for the transition firing can be breadth first or depth first searching for all possible (enabled) transitions and construct a directed graph with a transition as a node. In our implementation, we randomly choose one enabled transition and fire it. Each time only one enabled transition is fired because we choose interleaving semantics.

Although the generated code is a multithreaded object-oriented program, if we view each component class, the code for behavior model is executed sequentially in terms of each fired transition. Since we use interleaving semantics for the java construct, and we only choose the current path during the runtime checking, we can reduce a java construct for a SAM component/connector to a sequential object oriented program.

All AspectJ and ArchJava code are finally compiled to the java before executed, we can treat them as same as java code. Among component classes, the concurrent execution of multithreaded program is implemented. In this proof, we only consider each component/connector's behavior model - Petri net. Thus we did not discuss the concurrency in the multithreaded program. Besides, the synchronized communication among objects is used to reach the mutual exclusion. We simply choose a sequential object oriented assertion language that did not consider the synchronized methods. In our proof, we assume the correctness of the synchronized methods in the java program. Finally, the data observation modifiers (i.e. public, private, and protected) are not discussed and silently assume to be correct during the program execution. Based on these concerns and since we choose interleaving semantics, we simplify a java program for a component/connector's behavior model to a sequential program, and use a sequential assertion language to represent the java program for each component/connector. The syntax for assertion language is adopted from [170]. 


\section{An Assertion Language for Java Construct}

In this part we describe a programming language [170] (COORE) that embodies the object-oriented features and mainly resembles the features of the java construct output from SAM Parser. This assertion language supports single-inheritance and subtyping as in Java and $\mathrm{C \#}$. We use this syntax as an abstraction of our Java construct. The syntax of COORE only describe the sequential $\mathrm{OO}$ programming language, it cannot cover concurrent aspect used in Java construct. The following Table 8 is the syntax of COORE - a subset of java programming language.

Table 8: COORE Syntax of Sequential Java Construct [170]

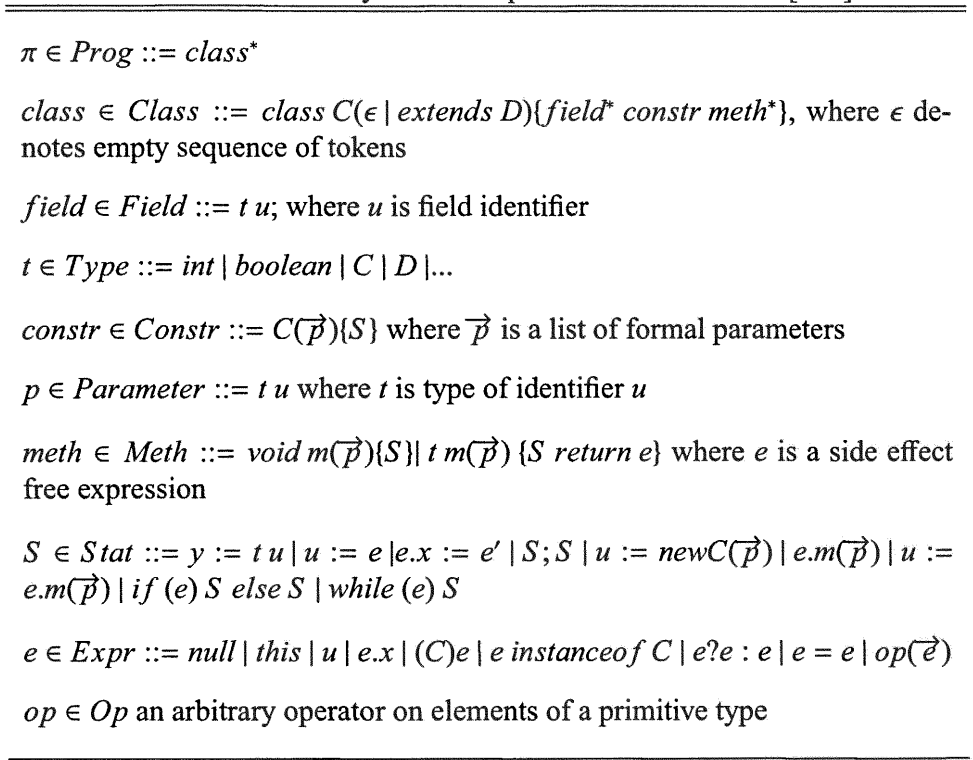

As shown in the Table 8, the exception handling is not included in the syntax. For our work, we only concern the features related with the model. Since exception handling is a general function provided for programming langauge, we do not discuss it. Secondly, interface classes and abstract classes are not covered in the above syntax. These features facilitate the programming especially in the object oriented program. However, they are not 
concerned in the high level behavior model, in this case, we simply assume their correctness in the java context. Finally, the main omission is concurrency. Since each component class is implemented as a sequential program using interleaving semantics, this syntax can describe the java construct of the SAM behavior model.

An operational semantics [169] for this assertion language is discussed as follows and given in the Table 12 and Table 10.

In this simple object oriented programming language, a state is captured by two aspects, a global state outside an object and a local state inside an object. A state is thus represented by a pair $(s k, h)$, where $s k$ is a local variable function (in [169] called stack), a total function that maps local variable to values, and $h$ is object state function (in [169] called heap), a partial function that maps each existing object to its internal state. Let $\mathcal{E} \llbracket e \rrbracket$ be the. evaluation on an expression $e$. The expression evaluation is shown in the Table 12.

In the Table 12, $\operatorname{origin}(C, x)$ yields the origin of field $x$ of an object of class $C$,

$$
\operatorname{origin}(C, x)= \begin{cases}C & \text { if } x \in \text { Fields }(C) \\ \operatorname{origin}(\operatorname{par}(C), x) & \text { otherwise }\end{cases}
$$

where $\operatorname{par}(C)$ denotes the parent class of class $\mathrm{C}$; it is undefined for class object. $\operatorname{dom}(t)$ denotes the value domain of type $t$. $[e]$ denotes the type of an expression $e$.

Additional rules of the operational semantics are given in the Table 10.

In Table 10, $h .[o \mapsto \operatorname{init}(C)]$ denotes the object state function that is obtained from $h$ by extending its domain with an object $o$ when $h(o)$ is undefined, $O C \triangleq \operatorname{constr}(C)$ (be the constructor method declaration in class $S), M C_{1} \triangleq u:=e_{0} . m\left(e_{1}, \ldots, e_{n}\right)\{S$ return $e\}$, $M C_{2} \triangleq m\left(p_{1}, \ldots, p_{n}\right)\{S\}$, If $\triangleq i f(e)\left\{S_{1}\right\}$ else $\left\{S_{2}\right\}$, and $W h \triangleq$ while $(e)\{S\}$. init $(t)$ denotes the default value of type $t$ according to following cases:

$$
\begin{aligned}
& \text { init(boolean) }=\text { false } \\
& \text { init(int })=0 \\
& \operatorname{init}(C)=\text { null }
\end{aligned}
$$




$$
\begin{aligned}
& \mathcal{E} \llbracket \operatorname{null} \rrbracket(s k, h)=\text { null } \\
& \mathcal{E} \llbracket u \rrbracket(s k, h)=s k(u) \\
& \mathcal{E} \llbracket e . x \rrbracket(s k, h)= \begin{cases}\perp & \text { if } v \in\{\text { null }, \perp\} \\
h(v)(\operatorname{origin}([e], x))(x) & \text { otherwise }\end{cases} \\
& \mathcal{E} \llbracket(C) e \rrbracket(s k, h)= \begin{cases}v & \text { if } v \in \operatorname{dom}(C) \\
\perp & \text { otherwise }\end{cases} \\
& \mathcal{E} \llbracket \text { e instanceof } C \rrbracket(s k, h)= \begin{cases}\perp & \text { if } v=\perp \\
\text { true } & \text { if } v \in \operatorname{dom}(C) \\
\text { false } & \text { otherwise }\end{cases} \\
& \mathcal{E} \llbracket e_{1} ? e_{2}: e_{3} \rrbracket(s k, h)= \begin{cases}\perp & \text { if } v_{1}=1 \\
v_{2} & \text { if } v_{1}=\text { true } \\
v_{3} & \text { if } v_{1}=\text { false }\end{cases} \\
& \mathcal{E} \llbracket e_{1}=e_{2} \rrbracket(s k, h)= \begin{cases}\text { true } & \text { if } v_{1}=v_{2} \\
\text { false } & \text { otherwise }\end{cases} \\
& \mathcal{E} \llbracket o p\left(e_{1}, \ldots, e_{n}\right) \rrbracket(s k, h)= \begin{cases}\perp & \text { if } v_{i}=\perp \\
o p\left(v_{1}, \ldots, v_{n}\right) & \text { otherwise }\end{cases}
\end{aligned}
$$

The first column is the evaluation on the statements, we use $\triangleq$ represent define, $L A \triangleq$ $\mathcal{E} \llbracket u:=e \rrbracket, F A \triangleq \varepsilon \varepsilon \llbracket e . x:=e^{\prime} \rrbracket$, and so on.

In the program defined in the COORE, we consider following as atomic actions:

1. Expression evaluation as atomic action. Thus the local variable set $y$ and $o p$ all atomic actions. In the semantics concern, we care about the elements that are related to those appear in the SAM model, markings, transitions that form the state sequence. In order to correctly implement these elements, objects are created and methods are invoked. Expression evaluation is one of the fundamental steps in these operations, which is related to the memory location, register allocation, etc.. We can simply assume it is correctly implemented without any interruption because it is far from 
being visualized in the high level model. Thus we consider it as an atomic action with regard to those high level elements that are related to SAM model.

2. We also consider assignments as atomic actions. Assignment is a notorious issue in the analysis of programming langauge semantics due to side effects. To avoid side effects in the multi-tasks environment, the assignment much be executed as an uninterruptible atomic action, so that only one task at a time can execute any part of it.

An object's observable communication trace can be defined by the history of all communication events between the object and its environment, which represents an abstract view of its state, readily available for reasoning about past and present behavior. Let $O b j$ be the set of object id, Meth be the set of methods, $S$ be the set of statements. We define communication events as follows.

Definition 9 (Communication Event) $A$ communication event of an object $o \in O b j$ (where Obj is the set of object id) is defined by a tuple $\left\langle o_{1}, o_{2}, m>\right.$ such that $o_{1}, o_{2} \in O b j$ and $o_{1} \neq o_{2}, m \in$ Meth.

Communication events are the ingredients of the communication trace, which is defined as follows.

Definition 10 (Communication Trace) Let Prog be the set of java construct program for a SAM component/connector translated by SAM Parser. Class is the set of classes defined in the Prog, Field, Meth are the set of fields and methods defined in a class class $\in$ Class respectively. We use lower case to represent elements in a set, e.g., a method meth $\in$ Meth.

The communication trace ct(Prog) in the java construct is composed of a sequence of communication events. We define the communication trace of java construct constructively in Table 11:

Next, we give an operational semantics [169] for this assertion language as follows. 
A state is represented by a pair $(s k, h)$, where $s k$ is a local variable function (in [169] called stack), a total function that maps local variable to values, and $h$ is object state function (in [169] called heap), a partial function that maps each existing object to its internal state. Let $\mathcal{E} \llbracket e \rrbracket$ be the evaluation on an expression $e$. We have the expression evaluation in the Table 12 .

In the Table 12, origin $(C, x)$ yields the origin of field $x$ of an object of class $C$,

$$
\operatorname{origin}(C, x)= \begin{cases}C & \text { if } x \in \text { Fields }(C) \\ \operatorname{origin}(\operatorname{par}(C), x) & \text { otherwise }\end{cases}
$$

where $\operatorname{par}(C)$ denotes the parent class of class $\mathrm{C}$; it is undefined for class object. $\operatorname{dom}(t)$ denotes the value domain of type $t .[e]$ denotes the type of an expression $e$.

\subsubsection{Mapping Relation}

During the execution of the java construct, each component class is instantiated as one object in the implementation. This indicates that there is only one instance for each component/connector in SAM model. In reality, in the java construct, following the above operational semantics for the sequential oo program, there are many internal methods, fields, assignments, expressions in the output communication trace. That is because we are working on the real program that contains many detailed execution steps. As shown in the example for the communication trace of transition $t_{7}$ in the component $U s e r$, we can say that the communication trace for even one fired transition can contain many information that is barely to matched the transition firing rule. In order to make the output communication trace of the java construct more concise and neat, we choose the key methods, fields and necessary expressions as a compact set for each necessary element in the SAM model. The elements in SAM model and the related compact set are listed in the Table 13.

As discussed before, a firing rule for a transition includes the preset and postset of the transition. If we fix an order on the places then after each firing of a transition, we can get the same sequence for the same transition under different markings except for the tokens 
in places. Each input place object is evaluated based on the index of the input place vector, the updating of the each output place object is done based on the index of the output place vector. We can use the index of vector as the order for places.

From Table 13, we define the mapping relation $f$ as a function that maps each element in the set of places $P$, set of transitions $T$, and tokens CONs in a PrT net $N$ to the corresponding entities in the communication trace. Let $e$ be an element defined in the set $E=P \cup T \cup C O N s$, we have $\forall e \in E, f(e) \in \operatorname{ct}(N)$. For instance, $f($ (uInfoReq_15") = $\operatorname{ct}\left(\right.$ (uInfoReq_15") $\in \operatorname{ct}\left(\right.$ Prog $\left._{U s e r}\right)$.

For one token structure, although we specify the sort of the token as a Set in java, it is implemented as a vector in java construct. The Vector class implements a growable array of objects. Like an array, it contains components that can be accessed using an integer index. However, the size of a Vector can grow or shrink as needed to accommodate adding and removing items after the Vector has been created [5]. As an object instantiated and evaluated in the java semantics, it is consistent with that of the one token structure.

The implementation of Set in java is not synchronized [5]. If multiple threads access a set concurrently, and at least one of the threads modifies the set, it must be synchronized externally. This is typically accomplished by synchronizing on some object that naturally encapsulates the set. Synchronizing objects will bring some notorious issues especially in the debugging. This happened for both TreeSet and HashSet. The second reason is that in most of cases the vector is enough for the implementation since vector can provide the searching and indexing functions. These functions are what we need in the coding. Thus in our functional code, we implemented this as vector.

\subsubsection{Correspondence}

To reflect the firing rule for each transition in the PrT net, it needs to extract the necessary information that is relative to the firing rule for each transition, i.e., the preset and postset of the transition as well as the transition (identification) itself. How to identify each transition and its pre- and postset on a communication trace is the first issue that needs to be solved. 
The projection concept on a communication trace can help in this case. In this work, first I define the projection on a communication trace generated from a PrT net.

Definition 11 (Projection on a Communication Trace) Given $a \operatorname{Pr} T$ net $N=$ (Net, S pec, Insc) with net structure defined as $N e t=(P, T, F)$, its communication trace is $\operatorname{ct}(N)$. Let $S$ be the set of elements defined the java construct, The projection Proj of communication trace $\operatorname{ct}(N)$ on the set $S, \operatorname{Proj}(c t(N), S)$, is the sequence arising from $\operatorname{ct}(N)$ when all literals not contained in $S$ are deleted.

For the SAM example with a component User and port id "uInfor_15", $\operatorname{Proj}($ ct(User $),\{$ “uInfor_15"\} $\}=$..."uInfor_15"..."uInfor_15"...).

Corollary 2 Given a PrT net $N=(N e t, S p e c$, Insc $)$ with net structure defined as Net $=$ $(P, T, F)$, its communication trace is ct $(N)$. Let $\left.S\right|_{t}$ be the set of preset and postset of transition $t$ w.r.t. the java construct, i.e.,

$$
\begin{aligned}
\left.S\right|_{t}= & \left\{<p_{r 1}, p_{r 1} \cdot \operatorname{contains}\left(t k_{r 1}\right)>, \ldots,<p_{r m}, p_{r m} \cdot \operatorname{contain}\left(t k_{r m}>\right),\right. \\
& <p_{o 1}, p_{o 1} \cdot \operatorname{contains}\left(t k_{o 1}\right)>, \ldots,<p_{o n}, p_{o n} \cdot \operatorname{contains}\left(t k_{o n}\right)>, \\
& <t, t \text {. fire }()>\}
\end{aligned}
$$

$\left.F\right|_{t}$ be the firing for transition $t$ defined in the section 3.1. $f$ be the mapping function defined in the section 3.3. We have Proj(ct $\left.(N),\left.S\right|_{t}\right)=f\left(\left.F\right|_{t}\right)$.

Finally, we can lift each transition firing rule in the Java construct to the state sequence for a PrT net in the SAM model.

Proposition 3 (Sequential Consistency) Given a PrT net $N=(N e t, S p e c$, Insc) with net structure defined as $N e t=(P, T, F)$, its communication trace is $\operatorname{ct}(N)$.

For each transition $t \in T$ of a restricted $\operatorname{Pr} T$ net $N$, the firing rule for the transition $t$ is denoted as $\left.F\right|_{t}$, let $\left.S^{N}\right|_{t}$ be the set of preset and postset of transition $t$ as well as transition $t$, i.e., $\left.S^{N}\right|_{t}=\left\{p_{r 1}, \ldots, p_{r m}, p_{o 1}, \ldots, p_{o n}, t\right\}, \sigma=M_{0} t_{0} M_{1} \ldots t_{n-1} M_{n}$ be a state sequence defined in Definition 2, then we have 
$\operatorname{Proj}\left(\sigma,\left.S^{N}\right|_{t}\right)=\left.F\right|_{t}$.

We say a java construct is semantically consistent with its high level PrT net model if $\operatorname{Proj}\left(\operatorname{ct}(N),\left.S\right|_{t}\right)=f\left(\operatorname{Proj}\left(\sigma,\left.S^{N}\right|_{t}\right)\right)$, where $f$ is the mapping function defined on the section 3.3.

Proof

From the firing for a transition $t$, based on the Corollary, we can derive that the proposition is true.

endProof.

\subsection{Discussion}

In our communication trace, we did not consider the object initiation and object completion, which was discussed in the work [112]. That work [112] is to proof the correctness of an $\mathrm{OO}$ programming language CORE, while our work assume these $\mathrm{OO}$ features are true silently.

\subsubsection{Interleaving Semantics vs. Causal Semantics}

There are generally three types for the dynamic behavior of a Petri net model [28]. The first one is called the interleaving semantics, which looks at the set of occurrence sequences of a model. The second one is called the causal semantics, which looks at all processes run simultaneously. The third one is called parallel semantics, which is a mixed version of the above two. It comprises the full set of behaviors including all interleavings and all causal processes. Whereas the causal semantics is favorized in Petri net theory [Rei86] because of its ability to handle concurrency, the area of applications is dominated by interleaving semantics because of their easy and straight-away definition. Usually, simulation tools for Petri nets generate totally ordered sequences of transition occurrences and thus are based on interleaving semantics. In terms of the Restricted PrT nets and its high level process nets, the causal semantics are the maximum set of all the process nets, while the interleaving semantics are the minimum set of the process nets. The equivalence between causal semantics and interleaving semantics of Petri nets can be proved by bisimulation 
among the set of the process nets. The idea is to associate to a causal firing sequence a linearizable occurrence net. Many works have been done on some types of Petri nets [28].

\subsubsection{Granularity}

It is not surprising that since the very beginning of the design of parallel algorithms and parallel computation formalisms, a great deal of research effort has been devoted to interpreting parallel computation in more familiar, sequential terms. The classical example of this "sequential way of thinking" in computer science is the nondeterministic sequential interleaving semantics of concurrent computation.

In this context, a natural question arises: Given a parallel computing model, can its (logically) concurrent execution always be replaced by such nondeterminism, so that any given concurrent behavior can be faithfully reproduced via an appropriate choice of a sequential interleaving? The answer is Yes, provided that we simulate concurrent execution via sequential nondeterministic interleaving at a sufficiently high level of granularity of the basic computational operations.

In the mapping relation, we regulated the expression and operations as atomic actions. Following question is "is that fine enough for the computation to simulate the behavior of SAM?". If we consider each value in the stack, the operations on the stack cannot be reflected in the mapping relation. In other words, we cannot find an image in the mapping function for these operations.

On the other side, if we consider a coarse communication trace in the implementation, for instance, we only consider the object instantiation during runtime execution. Then we cannot get the knowledge of the tokens related with a place. We proved that the sequential semantics of the java construct can capture the sequential behavior of Restricted PrT nets, so we can say that the granularity for the analysis of java construct is fine.

According to the granularity level of the Petri net specification of a software system, the values of the measures below may change. For instance, at the topmost granularity level, one might represent a whole system by means of a Petri net with a single transition, 
whose input places represent the initial states of the processes of the system, and whose output places represent the final states of the processes. At lower granularity levels, the same system may be represented by a Petri net with a large number of places, transitions, and arcs. In this chapter, we are here interested in the definition of measures for a Petri net specification, so we assume that the specification has already been written. It is the specifier's responsibility to write a specification in such a way as to represent the modeled software system accurately. The granularity issue becomes important when empirically validating these measures for the internal attributes against measures for external attributes. Sensible comparisons between different specifications require that only specifications at close granularity levels be compared.

\subsubsection{State-based vs. action-based}

The formalisms employed in sate space methods may be roughly divided into two categories, state-based and action-based methods. State-based methods include model checking of many temporal logics that use Kripke structures and similar models. Actionbased methods include many process algebraic methods, where Labeled Transition Systems (LTSs) are used as a model. State-based formalisms discuss the system properties on the global state of the system, whereas action-based formalisms talk about the interaction among the system components. More, action-based semantics is unsatisfiable because of incapability to express fairness [124]. This is why some properties may be harder to express using just one approach.

In the work of Lamport in 1985 [124], he pointed out the difference of the system property specification between these two methods. The discussion of the underlying reason of the benefits for each method was presented by simple examples. Different actions or events may trigger systems or processes to the states, while it is hard to identify the behaviors of these systems/processes since states are same in the state-based specification. Similarly, 
same action may reach the different states of systems or processes, while it would difficulty to identify the two processes since the actions are same by using only action-based specification.

In our work, The Petri nets' behavior is identified by the sequence of states with the transition firing ordered. This is the mixed version of both state- and action-based. We believe the above issues for the single approach can be avoided. However, the temporal logic is a state-based approach that is used to describe system properties based on the state changing. Since behavior is specified by the combined version, we can say that the property is true when the property is held by the system.

\subsection{Summary}

This chapter presented two approaches to the correctness proof on the semantic consistency for translation validation from software architecture specification translation to Maude program and object-oriented program (Java).

In the translation validation from SAM model to the Maude program, we construct a rewrite theory $\left(\mathcal{R}^{\mathcal{P J}}\right)$ for a given $\operatorname{Pr} T$ net $(N)$ stepwisely. For the constructed rewrite theory $\left(\mathcal{R}^{\mathcal{P J}}\right)$ the semantic consistency is proved on the interleaving semantic of the PrT net and the proof term $(\alpha)$ of the rewrite theory based on the inference rules. In this proof each component/connector of a SAM model is considered as a subsystem and described by a rewrite theory and a PrT net. The main advantage is the stepwise comparison between rewrite theory and PrT net is established. The future work can be the proof of the bisimulation between them.

In the translation validation from SAM model to the Java program, we described the translation validation in four steps - a restricted version of PrT nets with its interleaving semantics is provided, the communication trace for java construct is defined, a mapping relation is established between the restricted PrT nets and java construct, and a consistency proposition is derived. The main advantages are 1) the weak bisimulation can be derived 
due to stepwise sequence construction; and 2) a possible extension to the multithreaded programs can be applied for the same approach to prove the correctness of $O O$ programs. This translation validation approach has few restrictions. It does not consider the synchronization of multithreaded components since the formal proof is established based on a sequential java assertion language (COORE). Some reduction is made to fit for this java assertion language. Secondly, exceptions in the target code as well as their semantics are not considered due to the same reason as before. Many of our generated code have the exception handling, such try-catch block. In our discussion, this part is ignored to simplify the validation. Since these cause only programming language and implementation-oriented issues, which are not closed related with the architecture specification, it is reasonable to ignore the exceptions. Furthermore, the fairness of the communication trace for the java construct is not considered. Finally, the ability of our approach to check correctness of algebraic simplifications is limited by the power of the decision procedure employed by the SAM Parser tool we use. 
Table 10: Operational Semantics of COORE [169]

$L A$

$\mathcal{E} \llbracket e \rrbracket(s k, h) \neq \perp$

$<u:=e,(s k, h)>\rightarrow(s k[u \mapsto \mathcal{E} \llbracket e \rrbracket(s k, h)], h)$

FA

$\frac{\mathcal{E} \llbracket e \rrbracket(s k, h)=o \in\{\perp, \text { null }\} \mathcal{E} \llbracket e^{\prime} \rrbracket(s k, h)=v \neq \perp \operatorname{origin}([e], x)=D}{<e . x:=e^{\prime},(s, h)>\rightarrow\left(s, h\left[o . x_{D} \mapsto v\right]\right)}$

$o=(C$, id $) h(o)$ is undefined

$\mathcal{E} \llbracket e_{i} \rrbracket(s k, h) \neq \perp \forall i \in\{1 \ldots n\}$ constr $(C) \equiv C\left(p_{1}, \ldots, p_{n}\right)\{S\}$

$O C$

$<S,\left(\right.$ sk $\left[\right.$ this, $\left.\left.\left.p_{1}, \ldots, p_{n} \mapsto o, v_{1}, \ldots, v_{n}\right], h .[o \mapsto \operatorname{init}(C)]\right)\right\rangle \rightarrow\left(s k^{\prime}, h^{\prime}\right)$

$<u:=\operatorname{new} C\left(e_{1}, \ldots, e_{n}\right),(s k, h)>\rightarrow\left(s k[u \mapsto o], h^{\prime}\right)$

$\mathcal{E} \llbracket e_{0} \rrbracket(s k, h)=o=(C, i d)$

$\mathcal{E} \llbracket e_{i} \rrbracket(s k, h)=v_{i} \neq \perp, \forall i \in\{1 \ldots n\}$

$\operatorname{meth}(C, m) \equiv \operatorname{tm}\left(p_{1}, \ldots, p_{n}\right)\{S$ return $e\}$

$<S,\left(s k\left[t h i s, p_{1}, \ldots, p_{n} \mapsto o, v_{1}, \ldots, v_{n}\right], h\right)>\rightarrow\left(s k^{\prime}, h^{\prime}\right)$

$M C_{1}$ $\mathcal{E} \llbracket e \rrbracket\left(s k^{\prime}, h^{\prime}\right)=v \neq \perp$

$<u:=e_{0} \cdot m\left(e_{1}, \ldots, e_{n}\right),(s k, h)>\rightarrow\left(s k[u \mapsto v], h^{\prime}\right)$

$\mathcal{E} \llbracket e_{0} \rrbracket(s k, h)=o=(C, i d)$

$\mathcal{E} \llbracket e_{i} \rrbracket(s k, h)=v_{i} \neq \perp, \forall i \in\{1 \ldots n\}$

$\operatorname{meth}(C, m) \equiv \operatorname{tm}\left(p_{1}, \ldots, p_{n}\right)\{S$ return $e\}$

$M C_{2}$

$\frac{<S,\left(s k\left[t h i s, p_{1}, \ldots, p_{n} \mapsto o, v_{1}, \ldots, v_{n}\right], h\right)>\rightarrow\left(s k^{\prime}, h^{\prime}\right)}{<u:=e_{0} \cdot m\left(e_{1}, \ldots, e_{n}\right),(s k, h)>\rightarrow\left(s k, h^{\prime}\right)}$

$S_{1} ; S_{2} \quad \frac{\left\langle S_{1},(s k, h)>\rightarrow\left(s k^{\prime \prime}, h^{\prime \prime}\right)<S_{2},\left(s k^{\prime \prime}, h^{\prime \prime}\right)>\rightarrow\left(s k^{\prime}, h^{\prime}\right)\right.}{<S_{1} ; S_{2},(s k, h)>\rightarrow\left(s k^{\prime}, h^{\prime}\right)}$

$I f_{1} \quad \underline{E} \llbracket e \rrbracket(s k, h)=$ true $\left\langle S_{1},(s k, h)\right\rangle \rightarrow\left(s k^{\prime}, h^{\prime}\right)$

$<$ if $(e) S_{1}$ else $S_{2},(s k, h)>\rightarrow\left(s k^{\prime}, h^{\prime}\right)$

$I f_{2} \quad \frac{\mathcal{E} \llbracket e \rrbracket(s k, h)=\text { true }\left\langle S_{1},(s k, h)>\rightarrow\left(s k^{\prime}, h^{\prime}\right)\right.}{<i f(e) S_{1} \text { else } S_{2},(s k, h)>\rightarrow\left(s k^{\prime}, h^{\prime}\right)}$

$W h_{1} \quad \frac{\mathcal{E} \llbracket e \rrbracket(s k, h)=\text { false }}{<w h i l e(e) S,(s k, h)>\rightarrow(s k, h)}$

$\mathcal{E} \llbracket e \rrbracket(s k, h)=$ true

$<S,(s k, h)>\rightarrow\left(s k^{\prime \prime}, h^{\prime \prime}\right)$

$W h_{1}$

$\frac{<\text { while }(e) S,\left(s k^{\prime \prime}, h^{\prime \prime}\right)>\rightarrow\left(s k^{\prime}, h^{\prime}\right)}{<\text { while }(e) S,(s k, h)>\rightarrow\left(s k^{\prime}, h^{\prime}\right)}$ 
Table 11: Communication Trace

$\operatorname{ct}($ Prog $)=\operatorname{ct}($ class $*)$

$\operatorname{ct}($ class $)=\operatorname{ct}($ construct $)(\operatorname{ct}($ field $*) ; \operatorname{ct}($ meth $*))$,

where ; denotes possible interleaving order among meth $_{i}$ and field $_{i}$

$\operatorname{ct}($ construct $)=\operatorname{ct}(o=(C, i d)) \operatorname{ct}\left(e_{i}\right) \operatorname{ct}\left(p_{1}, \ldots, p_{n} \mapsto o, v_{1}, \ldots, v_{n}\right)$

$\operatorname{ct}($ field $*)=\operatorname{ct}\left(\right.$ field $\left._{1}\right) \ldots \operatorname{ct}\left(\right.$ field $\left._{n}\right)$, where $n \in N a t$

$c t(m e t h *)=\operatorname{ct}\left(\right.$ meth $\left._{1}\right) \ldots c t\left(m e t h_{n}\right)$, where $n \in N a t$

$\operatorname{ct}(\operatorname{meth}(C, m))=\operatorname{ct}(o=(C, i d)) \operatorname{ct}\left(e_{i}\right) \operatorname{ct}(S) \operatorname{ct}(e)$,

where $\operatorname{meth}(C, m) \equiv \operatorname{tm}\left(e_{1}, \ldots, e_{n}\right)\{S$ return $e\}$

$\operatorname{ct}(m e t h(C, m))=\operatorname{ct}(o=(C, i d)) \operatorname{ct}\left(e_{i}\right) \operatorname{ct}(S)$,

where $\operatorname{meth}(C, m) \equiv \operatorname{void} m\left(e_{1}, \ldots, e_{n}\right)\{S\}$

$\operatorname{ct}\left(i f(e) S_{1}\right.$ else $\left.S_{2}\right)=\operatorname{ct}(e)\left(\operatorname{ct}\left(S_{1}\right) \cup \operatorname{ct}\left(S_{2}\right)\right)$

$\operatorname{ct}($ while $(e) S)=(\operatorname{ct}(e) \operatorname{ct}(S)) *$

$\operatorname{ct}\left(S_{1} ; S_{2}\right)=\operatorname{ct}\left(S_{1}\right) \operatorname{ct}\left(S_{2}\right)$

$c t(u:=e \cdot m(\vec{e}))=\varepsilon \llbracket u:=e \cdot m(\vec{e}) \rrbracket c t(e \cdot m(\vec{e}))$

$\operatorname{ct}(u:=n e w C(\vec{e}))=\mathcal{E} \llbracket u:=\operatorname{new} C(\vec{e}) \rrbracket$

$\operatorname{ct}(u:=e)=\varepsilon \llbracket u:=e \rrbracket$

$\operatorname{ct}(e)=\mathcal{E} \llbracket e \rrbracket$ 
Table 12: Expression Semantics [169]

$$
\begin{gathered}
\mathcal{E} \llbracket e . x \rrbracket(s k, h)= \begin{cases}\perp & \text { if } v \in\{\text { null }, \perp\} \\
h(v)(\text { origin }([e], x))(x) & \text { otherwise }\end{cases} \\
\mathcal{E} \llbracket(C) e \rrbracket(s k, h)= \begin{cases}v & \text { if } v \in \operatorname{dom}(C) \\
\perp & \text { otherwise }\end{cases}
\end{gathered}
$$$$
\mathcal{E} \llbracket \text { e instanceof } C \rrbracket(s k, h)= \begin{cases}\perp & \text { if } v=\perp \\ \text { true } & \text { if } v \in \operatorname{dom}(C) \\ \text { false } & \text { otherwise }\end{cases}
$$

$$
\begin{gathered}
\mathcal{E} \llbracket e_{1} ? e_{2}: e_{3} \rrbracket(s k, h)= \begin{cases}\perp & \text { if } v_{1}=\perp \\
v_{2} & \text { if } v_{1}=\text { true } \\
v_{3} & \text { if } v_{1}=\text { false }\end{cases} \\
\mathcal{E} \llbracket e_{1}=e_{2} \rrbracket(s k, h)= \begin{cases}\text { true } & \text { if } v_{1}=v_{2} \\
\text { false } & \text { otherwise }\end{cases}
\end{gathered}
$$


Table 13: A Mapping Relation of Elements in Component/Connector of SAM model

\begin{tabular}{|c|c|c|}
\hline $\begin{array}{l}\text { SAM } \\
\text { Model }\end{array}$ & Java Construct & Communication Event \\
\hline $\begin{array}{l}\text { place } p_{i}\left(\alpha_{t}\right) \\
\text { transition } t_{i}\end{array}$ & $\begin{array}{l}\text { Object id } p_{i} \text { with } \\
\text { type of Place } \\
p_{i} \text {.contains }(t k) \\
\text { object id } t_{i} \text { with } \\
\text { type of Transition } \\
t_{i} \text {. fired() }\end{array}$ & $\begin{array}{l}<p_{i}, \text { contains }(\mathrm{tk})> \\
<t_{i}, \text { fired }()>\end{array}$ \\
\hline Guard $g$ & $\begin{array}{l}\text { Object if } g \text { with } \\
\text { type of Guard } \\
\text { g.guardEvaluate( } \\
\text { Vector pv) }\end{array}$ & $<$ g, guardEvaluate (Vectorpv) $>$ \\
\hline $\begin{array}{l}\text { int } \\
\text { String } \\
\text { boolean } \\
\text { Product }\end{array}$ & $\begin{array}{l}\text { int } \\
\text { String } \\
\text { boolean } \\
\text { user-defined class }\end{array}$ & $\begin{array}{l}\text { int } \\
\text { String } \\
\text { boolean } \\
\text { user-defined class }\end{array}$ \\
\hline
\end{tabular}




\section{CHAPTER 8}

\section{CASE STUdIES}

\subsection{Introduction}

We have realized and implemented the integrated framework on several different scale examples. This chapter will demonstrates the application of the integrated framework to these examples. First, we apply the integrated framework to a small but illustrative example - a coffee machine. Second, we apply the integrated framework to a network security protocol under attack scenario. Third case study is an online shopping example. This case study shows the advantages for the deadlock checking in the design level for the integrated framework. Finally, we check and monitor an embedded system - a cruise control example using this integrated framework. In addition, an extension of SAM model on the service oriented architecture (SOA) named SO-SAM is demonstrated with an example. Moreover, an UML architecture description for the web system connects the SO-SAM model with the integrated framework by an image processing example. This demonstrated the method from UML architecture description for the web system to the SO-SAM model and the verification through the integrated framework.

\subsection{Coffee Machine}

Our first running example is a coffee machine from [195]. Fig. 18 shows a simplified SAM model of coffee machine. In SAM, there should have a component CoffeeMachine, a composition CoffeeMachine, and a hierarchical mapping from the component to the composition. However, in order to make the figure more straightforward, we integrate these three parts and still call the composition CoffeeMachine. Thus, the composition CoffeeMachine has ports that actually belong to the component CoffeeMachine. The connection 
between a port of the composition and a port of its subcomponent is called glue, which is actually defined in the hierarchical mapping.

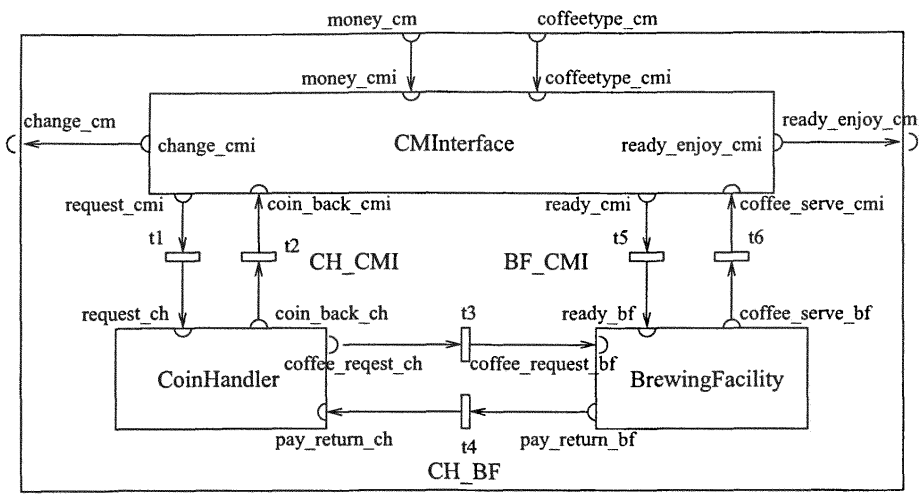

CoffeeMachine

Figure 18: SAM Model of Coffee Machine

From this figure, we can see the coffee machine itself is modelled as a composition CoffeeMachine, which has three sub components: CMInterface, CoinHandler, and BrewingFacility. Behavior models of these components are demonstrated in Fig. 19, Fig. 20, and Fig. 21.

Composition CoffeeMachine has four ports: money_cm, coffeetype_cm, change_cm, and ready_enjoy_cm, which are glued with ports in component CMInterface: money_cmi, coffeetype_cmi, change_cmi, and ready_enjoy_cmi respectively. A message in one port is immediately flowed to another port if they are glued. The direction of a message flow is determined by the direction of glued ports:incoming ports or outgoing ports. So whenever there is a message in the port money_cm, it is passed to the port money_cmi immediately.

Property specifications for each component/connector in SAM is defined by LTL formulae. Some heuristics of how to specify temporal properties are given in [97]. Following is a property of component CoffeeMachine:

$\square(($ money_cm $(80) \wedge$ coffeetype_cm $(2)) \longrightarrow$

$\diamond($ coin_back_cm $(80) \vee$ 


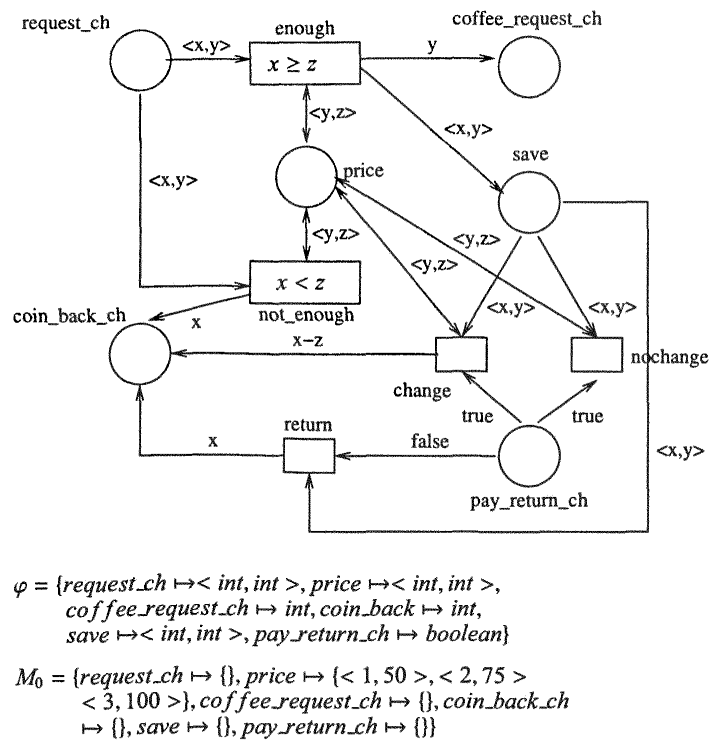

Figure 19: Behavior of Subcomponents - CoinHandler - in CoffeeMachine

$$
(\text { coin_back_cm }(5) \wedge \text { coffee_ready(1)))) }
$$

In the above formula, the atomic predicates specify the tokens contained in a port of a component. For example, atomic predicate money_cm $(80)$ is true if the port money_cm of component CoffeeMachine has a message 80. Since in SAM, a port refers to a unique place with the same name in the behavior model of the component, the atomic predicate also means the place money_cm contains a token 80 . Therefore, the above formula specifies the situation that when a customer inserts 80 cents into the coffee machine and chooses coffee type 2, the coffee machine either returns 80 cents or gives the customer 5 cents change and a cup of coffee.

This formula can be expressed in a more general way as follows, which means the user will either get his coffee and change or get his money back whenever he inserts coins and choose coffee type 2. The expression Port(money_cm actually specifies a type as the sort assigned to port money_cm. 


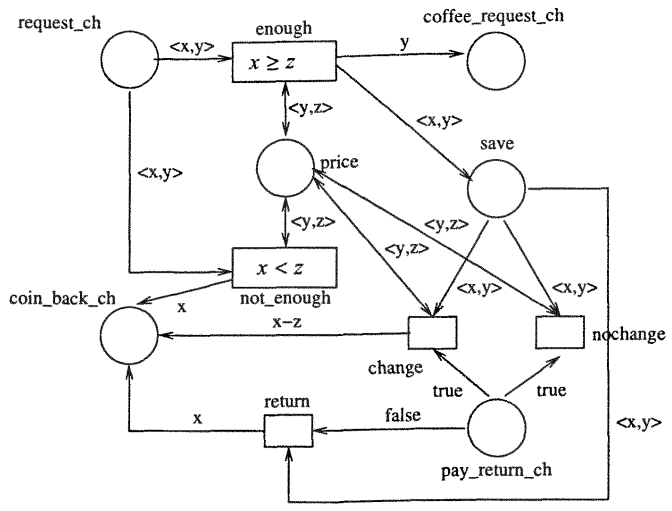

$$
\begin{gathered}
\varphi=\{\text { request_ch } \mapsto<\text { int, int }>\text {, price } \mapsto<\text { int, int }>, \\
\text { coffee_request_ch } \mapsto \text { int, coin_back } \mapsto \text { int }, \\
\text { save } \mapsto<\text { int, int }>\text {, pay_return_ch } \mapsto \text { boolean }\} \\
M_{0}=\{\text { request_ch } \mapsto\{\}, \text { price } \mapsto\{<1,50>,<2,75> \\
<3,100>\}, \text { coffee_request_ch } \mapsto\{\}, \text { coin_back_ch } \\
\mapsto\{\}, \text { save } \mapsto\{\}, \text { pay_return_ch } \mapsto\{\}\}
\end{gathered}
$$

Figure 20: Behavior of Subcomponents - BrewingFacility - in CoffeeMachine

$$
\begin{aligned}
& \forall m \in \text { Port }(\text { money_cm }), \square( \\
& (\text { money_cm }(m) \wedge \text { coffeetype_cm }(2)) \longrightarrow \\
& \diamond((\text { change_cm }(m) \wedge ! \text { ready_enjoy_cm }(1)) \vee \\
& \quad(\text { ready_enjoy_cm }(1) \wedge m>75 \\
& \quad \wedge \text { change_cm }(m-75)) \vee \\
& (\text { ready_enjoy_cm }(1) \wedge m==75)))
\end{aligned}
$$

In the work of property patterns [59], a set of categories is listed for the finite state verification of the non-timing systems based on more than five hundreds examples. Mainly there are two groups discussed - occurrence pattern and order pattern. Occurrence patterns talk about the occurrence of a given event/state during system execution, while order patterns talk about relative order in which multiple events/states occur during system execution. The supported formalisms of these patterns include LTL, CTL, GIL, QRE, and others. The following formula 3 is the application of the bounded existence pattern in the occurrence pattern group. 


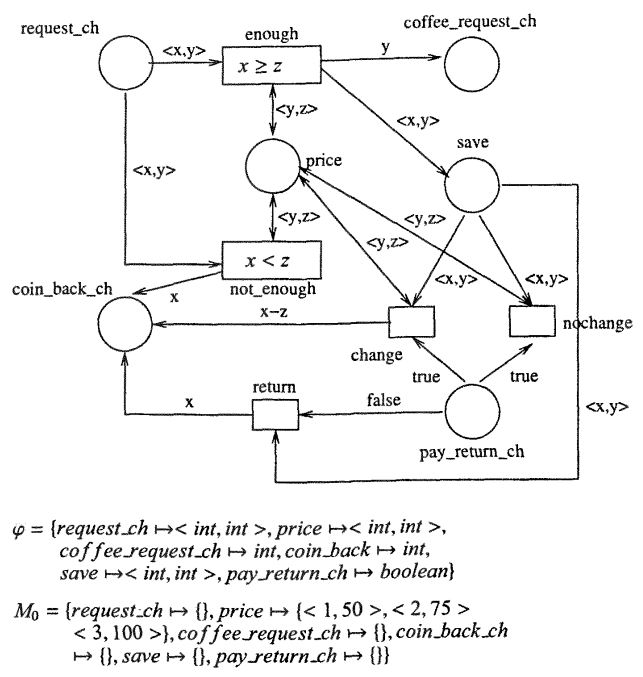

Figure 21: Behavior of Subcomponents - CMInterface - in CoffeeMachine

$$
\begin{aligned}
& \forall m, t \in \text { Port(coffee_request_ch), } \square( \\
& (\text { coffee_request_bf } f(t) \wedge \diamond \text { coffee_serve_cmi }(t)) \rightarrow \\
& ((\neg(\text { coffee_request_ch }(t) \wedge \neg \text { coffee_serve_cmi }(t)) \mathrm{U} \\
& (\text { coffee_serve_cmi }(t) \vee((\text { coffee_request_b } f(t) \wedge \neg \text { coffee_serve_cmi }(t)) U \\
& (\text { coffee_serve_cmi }(t) \vee((\neg \text { coffee_request_bf } f(t) \wedge \neg \text { coffee_serve_cmi }(t)) U \\
& (\text { coffee_serve_cmi }(t) \vee((\text { coffee_request_bf } f(t) \wedge \neg \text { coffee_serve_cmi }(t)) U \\
& (\text { coffee_serve_cmi }(t) \vee(\neg \text { coffee_request_b } f(t) \mathrm{U} \\
& \text { coffee_serve_cmi(t)) )) )) )) )) ) }
\end{aligned}
$$

\subsubsection{Model Checking}

All above properties are true after run on the Maude model checker. Besides, we also tried several properties specified in the constrained chain property patterns and response chain patterns [59]. The results can be returned within $1-4 \mathrm{~ms}$ at most. The main reason for the efficient result on the model checking of coffee machine example is this is a sequential system that the transition can be fired in order. The sort of port money_cmi can take a 
product token with two integer elements. Since there is not user identifications specified, whenever both integer elements match the pair defined in the place price, the transition enough can be fired, otherwise not_enough will be fired. This is corresponding to the ifcondition statements in the programming language, which is a sequential statement.

We define the size of the system using the number of process nets. Since process nets are defined on the initial markings, the size of the system $n$ can be defined as the number of initial tokens in the initiate place. For instance, in the coffee machine model, the size of the system can be specified by the number of initial tokens in the port money_cmi. In this experiment, when $n=4$ we cannot get the result. The system is running out of memory.

\subsubsection{Runtime Verification}

As a result of executing SAM parser, lots of files are generated to implement Petri nets and SAM. Table 14 shows the distribution of generated files. From this table, we can see even for this simple example, more than 200 java classes are generated to implement Petri nets. The reason for this is due to the most important principle for the SAM parser: The generated code is kept simple to understanding and modifying if necessary. In order to implement SAM, one ArchJava file is generated for each component, connector or composition. A component/connector class in ArchJava introduces several java classes, which are decided by the number of ports contained by the element. One thing we have to point out is that composition CoffeeMachine has no behavioral model. Its behavior is decided by its sub-components and sub-connectors.

Each fired transition is recorded by three parts: pre-condition, the transition name and id, and post-condition. For example, the following step means transition input is fired. As a result of the firing, token 85 in place money_cmi, and token 2 in place coffeetype_cmi are consumed, and token $<85,2>$ and 1 are added to place request_cmi and place sig respectively.

$<m o n e y \_c m i=\{85\}$, coffeetype_cmi $=\{2\}>$

-----Transition iutput(input)-----> 
Table 14: Summary of Generated Files

\begin{tabular}{|l|c|c|}
\hline \hline \# of Generated Files & For PN Implementation & For SAM Implementation \\
\hline CoffeeMachine & 0 & 1 \\
\hline CMInterface & 12 & 1 \\
\hline CoinHandler & 27 & 1 \\
\hline BrewingFacility & 16 & 1 \\
\hline CH_CMI & 6 & 1 \\
\hline CH_BF & 6 & 1 \\
\hline BF_CMI & 14 & 7 \\
\hline Templates & 1 & 0 \\
\hline Sort & 88 & 14 \\
\hline Total & 6 & 1 \\
\hline
\end{tabular}

$\langle$ request_cmi $=\{\langle 85,2\rangle\}, \operatorname{sig}=\{1\}\rangle$

During the experiment, the maximum number of the size of the system we checked is $\operatorname{six}(n=6)$. The result for the same formula can be output normally. The time and the size of system relation on the property 3 is listed in the Table 15.

\subsubsection{Discussion}

From the results of using model checking and runtime verification on the coffee machine example, we can summarize as follows.

1. In the small size of system, model checking is much more efficient. 
Table 15: Relation between Size and Time of Coffee Machine Example

\begin{tabular}{|l|l|}
\hline \hline System Size n & Time Consumed (ms) \\
\hline 1 & 3424 \\
\hline 2 & 3743 \\
\hline 3 & 3744 \\
\hline 4 & 4221 \\
\hline 5 & 4771 \\
\hline 6 & 4769 \\
\hline
\end{tabular}

2. When the model checker is out of space, runtime checker can still work well on properties.

3. The time consumption of the runtime checker is not linear to the size of system.

Although the time consumption of the runtime checker is not linear to the size of the system, it is still reasonable in terms of processors cost and resources for PC machine. When the program and the runtime checker are running on a machine, the performance for the system will be evaluated by the workload from monitor, storage, register, and others. These factors override the effects from the number of process nets because we only check the current execution path. In other words, in the multiprocessor machine, the performance of the runtime verification may not be able to improve efficiently due to the above factors. This also illustrates the reason that the time consumption of the runtime checker is not linear to the size of the system.

From the above summarization, we can say that runtime checker can be a complementary to the model checker when the size of the system is becoming huge. 


\subsection{Network Security Protocol Under Attack}

\subsubsection{Scenario Introduction}

E-commerce and enterprise systems can be secured in many different ways from simple use of password to digital authentication. State-to-state ENC (STS-ENC) protocol [57],a version of the authenticated Diffie-Hellman key agreement protocol [51], is to provide desirable security attributes in the network communication. The immunity is achieved by allowing the two parties to authenticate themselves to each other by the use of digital signatures and public-key certificates.

But this is not strong enough to immune from malicious attacks. Public key substitution attacks [31] on the protocol is an attack on a key agreement protocol that mislead one principal to false beliefs. An participant $A$ ends up believing she shares a key with $B$, and although this is in fact the case, $B$ mistakenly believes the key is instead shared with an entity $I \neg=A$. We use $A \hookrightarrow B$ to represent that A sends a message intended for $\mathrm{B}$, but intercepted by intruder $I$. This attacks can be described in the following message sequence (A for Alice, B for Bob and I for intruder Ink):

$$
\begin{aligned}
& \text { 1. } M 1: \mathrm{A} \hookrightarrow \mathrm{B} \quad \mathrm{A}, \alpha^{r A} \\
& \text { 2. } M 1^{\prime}: 1 \rightarrow \mathrm{B} \quad \mathrm{I}, \alpha^{r A} \\
& \text { 3. } M 2: \mathrm{B} \hookrightarrow \mathrm{A} \quad \operatorname{Cert}_{B}, \alpha^{r B}, E_{K}\left(S_{B}\left(\alpha^{r B}, \alpha^{r A}\right)\right) \\
& \text { 4. } M 2^{\prime}: \mathrm{I} \rightarrow \mathrm{A} \quad \operatorname{Cert}_{B}, \alpha^{r B}, E_{K}\left(S_{B}\left(\alpha^{r B}, \alpha^{r A}\right)\right) \\
& \text { 5. } M 3: \mathrm{A} \hookrightarrow \mathrm{B} \quad \operatorname{Cert}_{A}, E_{K}\left(S_{A}\left(\alpha^{r A}, \alpha^{r B}\right)\right) \\
& \text { 6. } M 3^{\prime}: \mathrm{I} \rightarrow \mathrm{B} \quad \operatorname{Cert}_{I}, E_{K}\left(S_{A}\left(\alpha^{r A}, \alpha^{r B}\right)\right)
\end{aligned}
$$

where $\operatorname{Cert}_{A}=\left(A, P_{A}, s_{T}\left\{A, P_{A}\right\}\right)$, is $A$ 's certificate containing $A$ 's identifying information, $A$ 's public key $P_{A}$, and a trusted authority $T$ 's signature $s_{T}$ over these information. $S_{A}(x)$ is $A$ 's signature on $x$ using her secret key. $E_{K}(M)$ is encryption over message $M$ using a symmetric key encryption scheme with key $K=\alpha^{r A \times r B}$, which is the ephemeral Diffie-Hellman shared secret key. $r A$ and $r B$ are the number picked up randomly by $\mathrm{A}$ and 
B respectively. In this message sequence, steps 1,3 , and 5 establish the normal communication between $A$ and $B$ without intruder.

The series of steps indicate an intruder Ink successfully impersonate Alice to communicate with Bob. The communication starts from Alice sending a message $M 1$ to Bob, which is intercepted by Ink. Intruder Ink uses $P_{A}$ as its own public key, to impersonate Alice to get the shared key with Bob. After Ink intercepts message $M 1$, he impersonates Alice and sends a modified message $M 1^{\prime}$ with his identifying information to Bob. When Ink receives the message $M 2$ from Bob, he sends message $M 2^{\prime}$ to Alice without modification. Thus when Alice sends out the message $M 3$, he intercepts and decrypts it with the shared key, and sends the faked message $M 3^{\prime}$ with his certificate to Bob.

\subsubsection{SAM Model of Network Security Protocol Under Attack}

The top level of SAM model of STS-ENC protocol under attack scenario is demonstrated in Fig. 22. There are one component TrustedAuthority, three compositions Alice, Intruder, and $B o b$ denoted by rectangles, and five connectors in this level. Due to space limit, the mappings from compositions to subcomponents and behavioral specifications are omitted. Because each connector behaves just like a data pipe, we simply explain components functionalities in the following.

The functionalities of component TrustedAuthority can be listed into the followings. First, given identifying information and a public key TrustedAuthority can calculate a certificate, second, TrustedAuthority can check if a certificate is valid or not by comparing the public and private keys based on the calculation. The composition Alice is responsible for sending message $M 1$, receiving message $M 2^{\prime}$, checking the validation of message $M 2^{\prime}$, and sending message $M 3$. The composition Intruder/Ink intercepts message $M 1$, substitutes identifying information, sends message $M 1^{\prime}$, intercepts message $M 2$, sends message $M 2^{\prime}$, intercepts message $M 3$, substitutes certificate and sends message $M 3^{\prime}$. The composition $B o b$ is responsible for receiving message $M 1^{\prime}$, sending message $M 2$, receiving message $M 3^{\prime}$, and checking the validation of message $M 3^{\prime}$ according to previous information. 


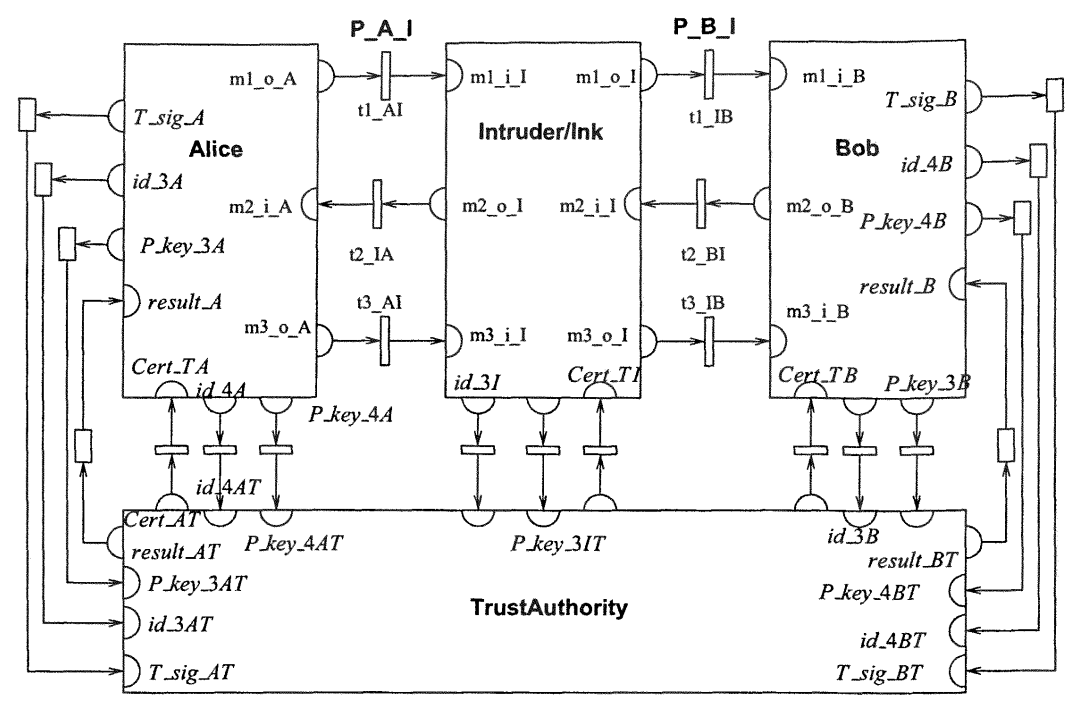

Figure 22: SAM Architecture of STS with Intruder

In SAM, components communicate with each other through ports represented as semicircles. An incoming port, represented by a semicircle inside of the component, only receives messages from other components at the same hierarchy, like port $m 2 \_i \_A$ of composition Alice in Fig. 22. Similarly, an outgoing port, represented by a semicircle outside of the component, only sends messages to other components, like port $m 2 \_o I$ of composition Intruder in Fig. 22. Therefore, composition Intruder can sends a message $M 2^{\prime}$ from the outgoing port $m 2_{-} o_{-} I$ to the incoming port $m 2_{-} i_{-} A$ of the composition Alice through the connector $P \_A I$.

\subsubsection{System Properties}

In SAM model, we have to make sure that compositions Alice, Ink, and Bob behave as expected from STS-ENC protocol. In other words, composition Alice first sends message $M 1$, then receives message $M 1^{\prime}$, and finally sends message $M 3$. This can be expressed by the following formulae on composition Alice: 


$$
\begin{aligned}
& \diamond\left(m 3 \_o \_A\left(<\operatorname{Cert}_{B}, E_{K}\left(S_{A}\left(\alpha^{r A}, \alpha^{r B}\right)\right)>\right)\right) \\
& {[*]\left(m 2 \_i \_A\left(<\operatorname{Cert}_{B}, \alpha^{r}, E_{K}\left(S_{B}\left(\alpha^{r B}, \alpha^{r A}\right)\right)>\right) \rightarrow\right.} \\
& \left.\langle *\rangle\left(m 1 \_o-A\left(<A, \alpha^{r A}>\right)\right)\right) \\
& {[*]\left(m 3 \_o \_A\left(<\operatorname{Cert}_{B}, E_{K}\left(S_{A}\left(\alpha^{r A}, \alpha^{r B}\right)\right)>\right) \rightarrow\right.} \\
& \left.\quad\langle *\rangle\left(m 2 \_i \_A\left(<\operatorname{Cert}_{B}, \alpha^{r B}, E_{K}\left(S_{B}\left(\alpha^{r B}, \alpha^{r A}\right)\right)>\right)\right)\right)
\end{aligned}
$$

The atomic predicate in above formula is in the form $\operatorname{Port}(m)$, which is evaluated true if specified port contains the message $m$. For example, predicate $m 1_{-} \_\_A\left(\left\langle A, \alpha^{r A}\right\rangle\right)$ is true if the port $m 1 \_o \_A$ of composition Alice has a message $\left\langle A, \alpha^{r A}\right\rangle$. Our work supports future time linear temporal logic and past time linear temporal logic. Formula 8 is a future time LTL formula, while formulae 5 and 6 are past time LTL. In above formulae, $\diamond,[*]$, and $\langle *\rangle$ are future time operator eventually, past time operator SometimeInThePast (sometime in the past), and AlwaysInThePast (always in the past) respectively.

Similarly, we can specify formulae for compositions $B o b$ and Ink to guarantee the correctness of their behavior. In addition to these formulae, another formula

$$
\diamond(\text { Succ(true }))
$$

on composition $B o b$ is defined to indicate if STS-ENC protocol can be attacked by the public key substitution attack, where Succ is a outgoing port of a sub component of composition Bob. In other words, if formula 7 is satisfied, it means Alice thinks she shares a key with $B o b$, but Bob thinks he shares a key with third party Ink.

\subsubsection{Model Checking}

All above properties are true after run on the Maude model checker. Besides, we also tried several properties specified in the constrained chain property patterns and response chain patterns [59]. The results can be returned within $0-8 \mathrm{~ms}$. 


\subsubsection{Runtime Verification}

The SAM model of STS-ENC protocol totally has 3 compositions, 16 components, 20 connectors, 312 ports, and 36 high level Petri nets with 290 places, 116 transitions and 331 arcs. It takes about 10 seconds for the SAM parser to generate the implementation of SAM model of STS-ENC protocol on a P4 $2.4 \mathrm{Ghz}$ machine with 512MB RAM. The generated implementation has 491 files, and it is executable without any modification. Most of them (419) is the implementation of components or connectors behavior (Petri nets). The reason of generating so many files is due to the most important principle for the SAM parser: We have to make the generated code easy to understand and minimize the cost of modification. It takes about 96 seconds for the generated implementation to execute and verify above 10 formulae. The execution of the generated implementation fires transition 114 times, i.e. almost one transition is fired every second. Most of the time is spending on the searching of the enabled transition and valid assignments to variables. Comparing the time consuming in the model checking method, the runtime verification is not efficient enough. However, the code can be manually optimized for critical transitions by overriding methods that judge if a transition is enabled. Most important is the runtime verification does not have state explosion problem with the large systems. The analysis of the formulae is simply discussed in the following.

From the $\log$ file generated in the execution of the implementation, the formula 7 is true, which means that the well-known public key substitution attacks on STS-ENC protocol is viable. In other words, Alice wants to share a public key with Bob. But in fact, Bob shares a public key with Ink. From the log file, we also can see that the formula 8 holds. However, the evaluations of formulae 5 and 6 are neither true nor false. This seems strange at first since the purpose of runtime verification was to check if formulae are satisfied or not. However this result is correct because these two formulae are past time LTL, which are supposed to be always satisfied. In other words, runtime verification code only reports exception for past time LTL formulae if it is violated, just like code in Fig. 14. Therefore, the unsure results for formulae 5 and 6 indicate that there are no violation detected. This 
means these formulae hold during the program execution, which assures the behavioral correctness of Alice, Ink, and Bob.

\subsection{Online Shopping}

\subsubsection{Scenario Introduction}

In this section we present an application of this approach to the basic electronic commerce process of online shopping with credit card transaction [74]. However, this approach is domain independent, i.e. SAM Parser does not depend on a particular domain of discourse but rather on fundamental definitions of an ontology that captures what a component and a connector are. The online shopping scenario is a well familiar scenario that used for many companies for their online sale systems. Different systems have different GUI, but they share the same functionalities. The Fig. 23 is a simple description of the online shopping scenario example.

Fig. 23, adapted from [6] and [204], describes online shopping processing in a free natural language, while an accompanying pictorial scheme provides the details of electronic shopping that precedes the payment processing.

The description in Fig. 23 concerns details of the shop system, which is the focus of the e-business depicted in [204]. The two modules - the front platform for the user for the graphically and textually representation of shopping operations and the back platform for the retailer for organization and billing, etc. are separated into 5 blocks of services Customer, ShoppingCart, Warehouse, OrderProcessing, and CreditCardProcessing. The retailer bank is a simple entity in the online shopping system and we do not concern the black box financial organization of business company. In what follows, we examine the SAM specification constructed, translated code and verification process to study several unique features of SAM Parser.

\subsubsection{SAM Model of Online Shopping System}

The top topology of an online shopping system specified in SAM model is demonstrated in Fig. 24. Each description block is captured in a component (or a composition). The 


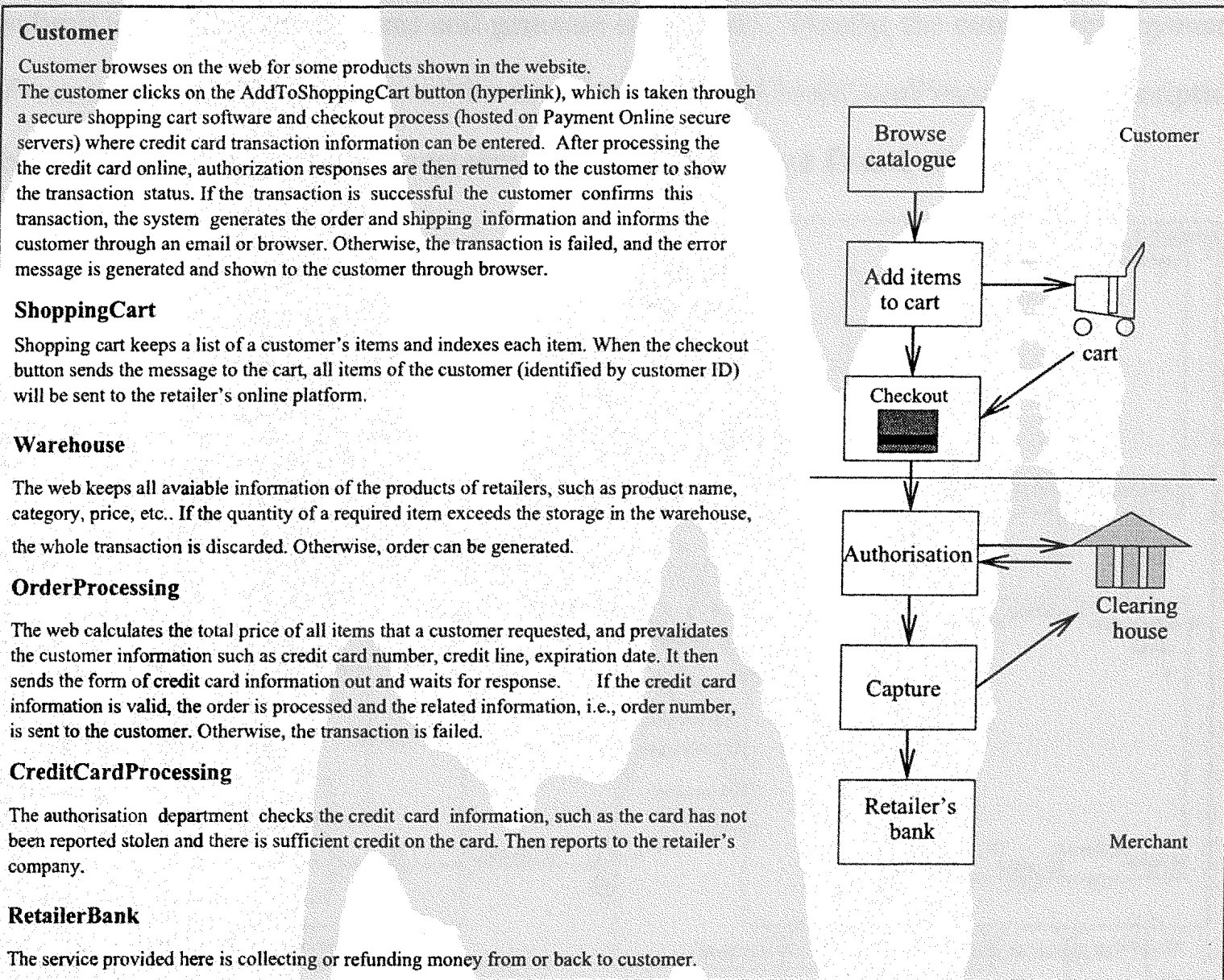

Figure 23: Natural language description of electronic shopping System

compositions $_{v}$ (different concept from the composition in SAM, we subscript with a letter v) between e-services are defined by connectors. Vertically, components/compositions in a lower level can form a composition in the upper level. For instance, block Customer and ShoppingCart is modeled in the component Customer and component Cart, and both components form a composition User in the topology of SAM. In the composition User, a customer can browse the web, choose category, select and checkout items. In addition, a user can also adjust the order and cart list. All these internal behaviors are represented by the refined inner components or Petri nets. Description blocks Warehouse and OrderProcessing are specified in the component Warehouse and Order, which form another composition E-Company. An E-company can evaluate user requests, confirm or reject user requests, 
submit user information forms and generate orders, etc.. Finally, the component PaymentProcess captures the behavior of the description block CreditCardProcessing. Description block RetailerBank is simply represented in the component RetailerBank.

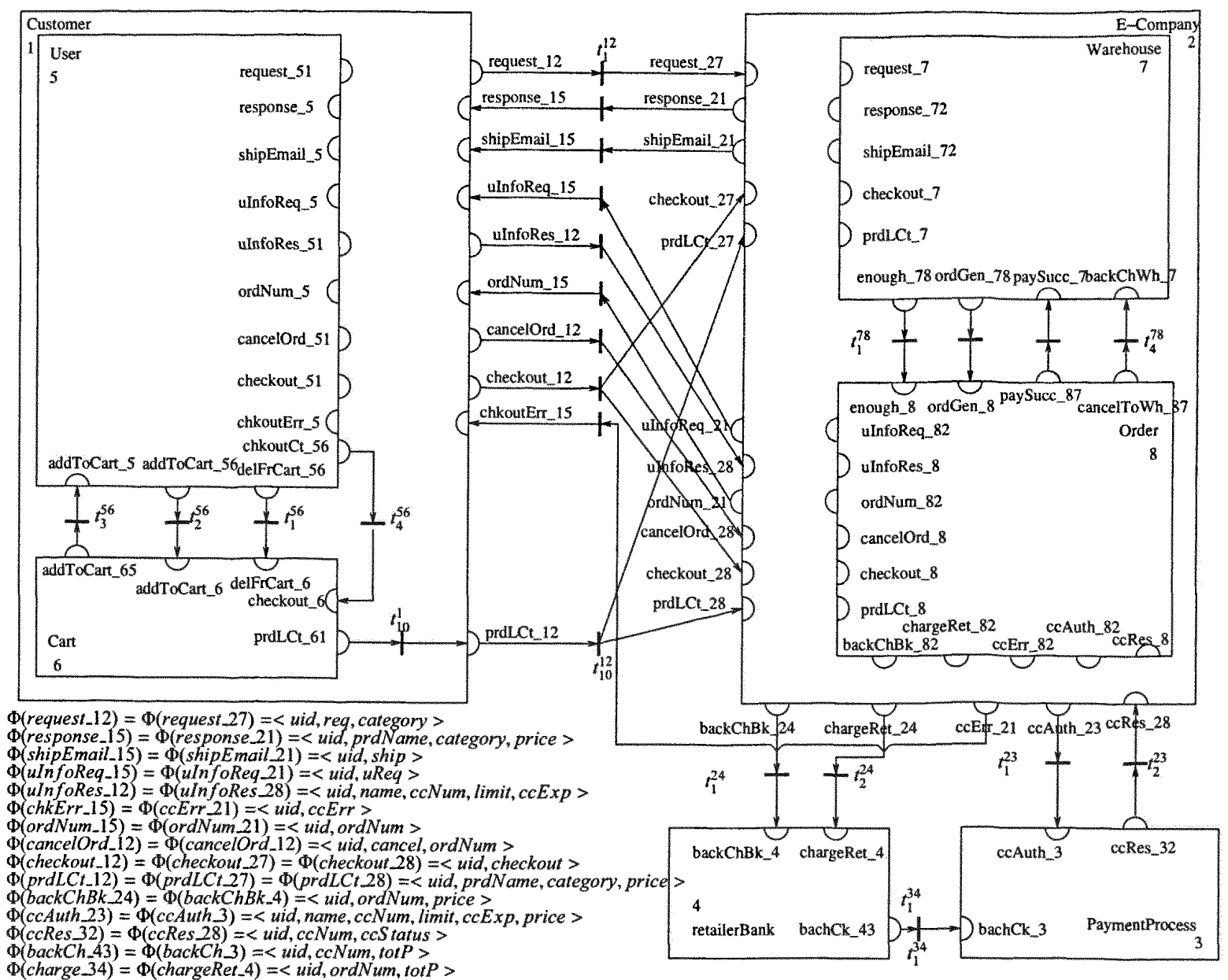

Figure 24: SAM Architecture of online shopping scenario

In SAM, components communicate with each other through ports represented as semicircles. An incoming port, represented by a semicircle inside of the component, only receives messages from other components at the same hierarchy, like port response_15 of composition User in Fig. 24. Similarly, an outgoing port, represented by a semicircle outside of the component, only sends messages to other components, like port response 21 of composition E-Company in Fig. 24. Therefore, composition E-Company can sends a message $r^{\prime}$ from the outgoing port response 21 to the incoming port response_15 of the composition 
Customer through the connector 12. Therefore, ports in SAM pass messages that are required or demanded by a service. These passing messages are used to describe the service composition $_{v}$.

In SAM, connector behaves as a communication channel between components. Ports gear a connector and a component together - an outgoing port in a component is an incoming port in the communicated connector. In this case, connector is a pipe to convey the data between services and a link to compose different service component. This behavior is defined by a transition connected between an incoming and an outgoing port. The whole version of SAM model of online shopping example is shown in the Appendix F.

\subsubsection{System Properties}

The basic requirements for the online shopping system are correctness, robustness and reliability. Since in this dissertation, the correctness property is the only concern of the software systems, all the system properties of each example listed is derived based on the correctness. For instance, if a user request to browse a product, a proper statement for the product is shown up; if a user put a "checkout" requirement, either (s)he gets the order information or is rejected because of payment issues (invalid credit cards, incorrect passwords, system errors, and others), in the later case, an error message is popped up to the user. In SAM model, we have to make sure that compositions User, and E-Company behave as expected from description in Fig. 23. In other words, if the composition Customer obtains a message $m 2=<$ uid, pname, category, price $>$ in the port response_15( $m 2)$, it indicates that sometime before the customer sent out a message $m 1=<$ uid, pReq, category $>$ through port request_12( $\mathrm{m} 1)$. This can be expressed by the following formulae on the composition Customer:

$$
\begin{aligned}
& \diamond(\text { response_15(< uid, pname, category, price }>)) \\
& \square(\text { request_12 }(<\text { uid, "pReq", category }>) \rightarrow \\
& \diamond(\text { response_15(<uid, pname, category, price }>)))
\end{aligned}
$$


The atomic predicate in above formula is in the form Port $(m)$, which is evaluated true if specified port contains the message $m$. For example, predicate request_12 $(<$ uid, pReq,category $>$ ) is true if the port request_12 of composition User has a message $<$ uid,pReq,category $>$. Our work supports future time linear temporal logic and past time linear temporal logic. Formula 8 is a future time LTL formula, while formula 9 is a past time LTL. In above formulae, $\diamond, \odot$, and $\diamond$ are future time operator eventually, and past time operator SometimeInThePast (sometime in the past), and AlwaysInThePast (always in the past) respectively.

There are many key system properties need to be enforced. We list three of them for the composition Customer and E-Company as follows.

1. Selective response property. When a customer sends a checkout request with products (s)he wants, eventually (s)he gets either an order and shipment information or the error response on this payment process. The formula is

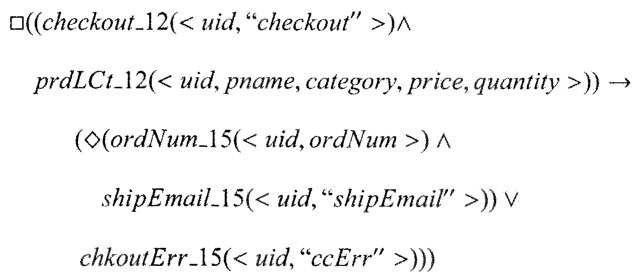

2. Conjoined reverse reasoning property. This property is to reason reversely from the current to past about the result. The features for this property is 1) it started with the current state which is true; 2) it is a past time temporal logic formula; 3) it involves several components and their relation is conjunction. For instance, when a customer receives an order information, we can reversely deduce that first the customer got a response on a request for the product(s), second the customer provided his/her credit 
card information sometime before, finally the customer got the shipment information. The LTL formula for this property is

$$
\begin{aligned}
& \square((\text { ordNum_15(<uid, ordNum }>) \rightarrow \diamond((\text { request_12 }(<\text { uid, "pReq", category }>) \rightarrow \\
& \diamond \text { response_15 }(<\text { uid, pname, category, price }>)) \wedge \diamond \text { shipEmail_15 }(<\text { uid, "shipEmail" }>) \wedge \\
& (\text { uInfoReq_15 }(<\text { uid, "uReq" }>) \rightarrow \diamond \text { uInfoRes_12 }(<\text { uid, name, ccNum, limit,ccExp }>)))))
\end{aligned}
$$

3. Cause-and-effect property. This involves more than two components/compositions, and there is a timing order between each two. For instance, the E-Company request to authorize a customer's payment information (credit card form). After component PaymentProcess responses, the E-Company sends out either an order and shipment information or the credit card error information to the component Customer. The LTL formula for this property is

$$
\begin{aligned}
& \square(\text { ccAuth_23 }(<\text { uid, name, ccNum, limit,ccExp, tprice }>) \rightarrow \\
& \diamond(\text { ccRes_28 }(<\text { uid,ccStatus }>) \rightarrow \\
& \diamond(\text { ccErr_21(<uid, "ccErr" }>) \vee \\
& (\text { shipEmail_15(<uid, "shipEmail" }>) \wedge \\
& \text { ordNum_21(<uid, ordNum }>)))))
\end{aligned}
$$

\subsubsection{Model Checking}

The basic requirements for the online shopping system are correctness, robustness and reliability. We use model checker of Maude [47] to validate the SAM model of the online shopping example against its properties. Before experiment, we translate the SAM model to the Maude program. The translation algorithm is shown in the Chapter 7 . The translated code is shown in the Appendix B. 
The results output from Maude are true for all the above formulae. Most the above formulae are liveness properties. We do not have deadlock freedom properties in this case. However, we did detect a deadlock in the model. The deadlock was located in the component Order, which is in the E-Company service, from the ccPrevalid place to the ccAuth port. After we fix the deadlock problem all properties are verified. The interesting readers can refer to paper [77] for details.

Besides, we also tried several properties specified in the constrained chain property patterns and response chain patterns [59]. The results can be returned within $0-13 \mathrm{~ms}$. When the size of the system $n=5$ we cannot get the result. The system is running out of memory.

\subsubsection{Runtime Verification}

The translation of SAM model and generated code of verification takes about 1.5 seconds for the SAM parser on a P4 2.4Ghz machine with 512MB RAM. The generated implementation has 280 files, and it is executable without any modification. Most of files are the implementation of components or connectors behavior (Petri nets). The statistic data is shown in Table 16. The reason of generating so many files is due to the most important principle for the SAM parser: We have to make the generated code easy to understand and minimize the cost of modification. It takes about 9.6 seconds for the generated implementation to execute and verify 8 formulae involved four compositions or components. The execution of the generated implementation fires transition 31 times, i.e. almost one transition is fired every second. Most of the time is spend on the search of enabled transition and valid assignments to variables. The code can be manually optimized for critical transitions by overriding methods that judge if a transition is enabled.

From the $\log$ file, we also can see that the formulae $8,10,12$ holds. However, the evaluations of formulae 9 is neither true nor false. This seems strange at first since the purpose of runtime verification was to check if formula are satisfied or not. However this result is correct because these two formulae are past time LTL, which are supposed to be always satisfied. In other words, runtime verification code only reports exception for past 
Table 16: Statistic Data of Generated Files

\begin{tabular}{|l|l|l|l|l|l|l|}
\hline $\begin{array}{l}\text { ArchJava } \\
\text { Files }\end{array}$ & $\begin{array}{l}\text { AspectJ } \\
\text { Files }\end{array}$ & $\begin{array}{l}\text { Java } \\
\text { Files }\end{array}$ & Properties & Types & Folders & $\begin{array}{l}\text { Total } \\
\text { Files }\end{array}$ \\
\hline 14 & 6 & 273 & 8 & 17 & 30 & 291 \\
\hline
\end{tabular}

time LTL formulae if it is violated, just like code in Fig. 14. Therefore, the unsure result for formula 9 indicate that there are no violation detected. Same reason for the formula 11. This means these formulae hold during the program execution, which assures the behavioral correctness of Customer, E-Company, and PaymentProcess.

During the experiment, the maximum number of the size of the system we checked is six $(n=6)$. The result for the same formula can be output normally. The time and the size of system relation on the property 12 is listed in the Table 17.

Table 17: Relation between Size and Time of Online Shopping Example

\begin{tabular}{|l|l|}
\hline \hline System Size n & Time Consumed (ms) \\
\hline 1 & 3117 \\
\hline 2 & 3434 \\
\hline 3 & 3449 \\
\hline 4 & 3771 \\
\hline 5 & 4111 \\
\hline 6 & 4219 \\
\hline
\end{tabular}




\subsection{Cruise Control System}

\subsubsection{Scenario Introduction}

This section introduces the case study cruise control system. It deals with a simplified cruise control (CC) example adapted from [85]. The purpose of a cruise control system [85] is to accurately maintain the driver's desired set speed, without intervention from the driver, by actuating the throttle-accelerator pedal linkage.

We assume an automatic transmission vehicle. When turned on by the driver, a cruisecontrol system (CCS) automatically maintains the speed of a car over varying terrain. The CCS can be turned on by pressing Start button, and enabled by pressing SetSpeed button. Resume button will enable the CCS at the last maintained speed when the brake is released. The cruise control function is disabled when the brake or accelerator pedal is pressed. Pressed once Resume button can increase the speed with $1 \mathrm{mph}$ and the SetSpeed button can decrease the speed with $1 \mathrm{mph}$ when the cruise control function is enabled. The cruise control system should be automatically disabled when the speed is below $25 \mathrm{mph}$ and above $90 \mathrm{mph}$. For the detail information, please refer to [76].

\subsubsection{SAM Model of CC System}

The top topology of an Cruise Control system specified in SAM model is given in Fig. 25. In the Fig. 25, we do not label every entity in the model because of visibility issue and space limitation. The labeled elements are those necessary in the presentation. In this model we describe the resuming, cruising, accelerating and decelerating states in the composition CruiseController, other behaviors, such as actuator, sensor are captured in other components or composition. Some component might be very simple, such as Engine, we only modelled the data flowing or repository in that component.

\subsubsection{Properties of the System}

After studying models and the errors discovered during the model validation, two main property categories have been selected:

1. Structural properties: this kind of properties is closely related to the topology of 


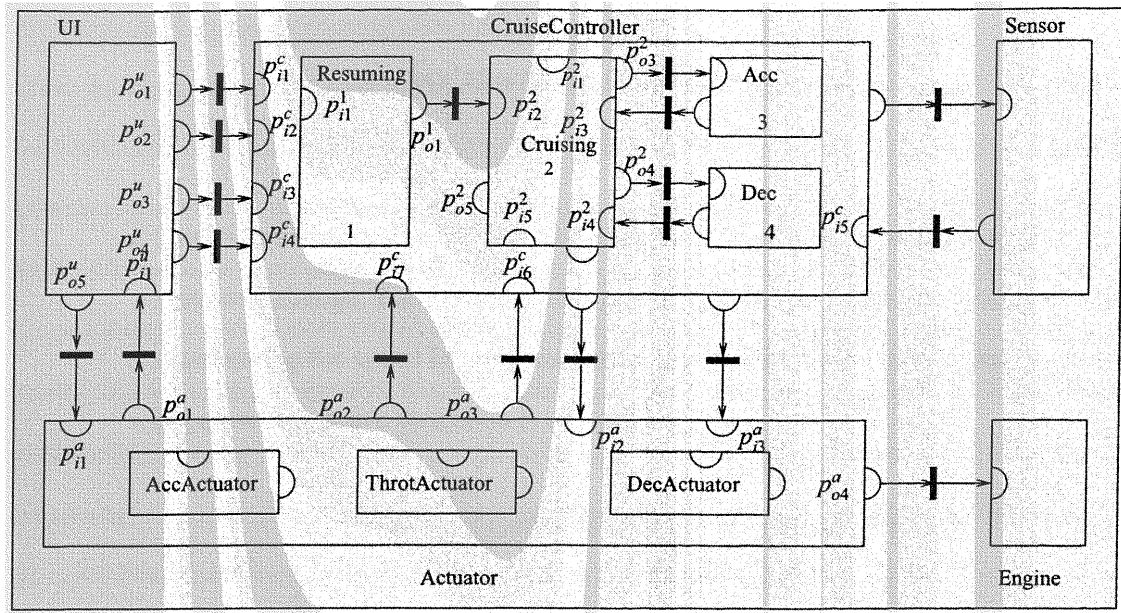

Figure 25: SAM Model of Cruise Control System

the model. These properties can be directly verified on the SAM model without animating the execution. These properties are necessary conditions that ensure the feasibility of the software. If one of them is not fulfilled, we can assert firmly that the system will not behave well.

2. Behavior properties: the dynamic feature of these properties means that they are related to state changing of the system. The evaluation of the dynamic properties are based on the behavior description - Petri nets. Its verification is achieved on a set of places describing a possible evolution of the system. Among these properties, we cite the following properties:

- Deadlock freedom checks whether an instance will stay in a state forever. If that case happens, the deadlock occurs and the system cannot program normally. For instance, whenever you request for resuming, the last maintained speed is retrieved and finally the system maintain the car at the last speed and set the last speed as current speed. 


$$
\square\left(p_{i 1}^{1}(\text { LastS peed }) \rightarrow \diamond p_{o 1}^{1}(\text { CurrentS peed })\right)
$$

- Livelock freedom checks different transitions can finally be fired so that the state will change. For instance, after pressing Resume button, the system either accelerating the speed by $1 \mathrm{mph}$ or retrieving the last maintained speed depending on which guard condition is satisfied, e.g.,

$$
\square\left(p_{i 2}^{2}(s p d) \rightarrow\left(p_{o 3}^{2}(s p d, \text { cruiseStatus }) \vee p_{o 5}^{2}(s p d, \text { cruiseStatus })\right)\right)
$$

3. Nonfunctional properties: In the realm of embedded systems, the most important non-functional properties are energy, timeliness, and dependability. In this case, we only consider some dependency properties.

\subsubsection{Runtime Verification}

The SAM model of Cruise Control System totally has 3 compositions, 10 components, 11 connectors. It takes about 4 seconds for the SAM parser to generate the implementation of SAM model of Cruise Control System on a P4 2.4Ghz machine with 512MB RAM. The generated implementation has over 100 files, and it is executable without any modification. Most of them is the implementation of components or connectors behavior (Petri nets). The reason of generating so many files is due to the most important principle for the SAM parser: We have to make the generated code easy to understand and minimize the cost of modification. It takes about 56 seconds for the generated implementation to execute and verify 8 formulae. The execution of the generated implementation fires transition 114 times, i.e. almost one transition is fired every second. Most of the time is spend on the search of enabled transition and valid assignments to variables. The code can be manually optimized for critical transitions by overriding methods that judge if a transition is enabled. 
From the $\log$ file generated in the execution of the implementation, the formula 13 is true, which means that whenever the resuming signal comes the cruise control status will be entered finally and the system maintains last speed. From the log file, we also can see that the formula 14 holds. However, the evaluations of some formulae are neither true nor false. This seems strange at first since the purpose of runtime verification was to check if formulae are satisfied or not. However this result is correct because those formulae are past time LTL, which are supposed to be always satisfied. In other words, runtime verification code only reports exception for past time LTL formulae if it is violated.

\subsection{Service Oriented Software Architecture Model (SO-SAM)}

Web services are emerging as a promising technology for the effective automation of inter-organizational interactions. By web service, we mean a self-contained, internetenabled application capable not only of performing business activities on its own, but also possessing the ability to engage other web services in order to complete higher-order business transactions.

A web service-oriented system refers to a system that integrates multiple web services automatically and transparently. The basic principles of service oriented architectures (SOAs) consist in modularizing functions and exposing them as services, which are typically specified using standard languages and interoperate through standard protocols. While several established and emerging standards bodies (e.g., $[1,2,7,9,10]$ and others) are rapidly laying out the foundations that the industry will be built upon, there are many research challenges behind web services that are less well-defined and understood [9] for the large number of web service application design and development.

SOAs will not scale without significant mechanization of service discovery, service adaptation, service composition, and service monitoring; as well as data, protocol, and process mediation. Recent efforts around service-oriented architectures provide even increased urgency for the above mentioned fundamental requirements for any scalable and economic software solution. In parallel, the textual specification is not suitable for formal methods 
such as computer aided verification. With computer aided verification, in particular model checking, it would be possible to decide crucial properties such as dependability of transactions, composability of processes, soundness, etc.. For a formal treatment, it is necessary to resolve the ambiguities and inconsistencies of the language which occurred particularly due to the hierarchical (esp. bottom-up) design and the unification of rather different services in the composition.

Currently most research focuses on the integration and automatic composition of web services but pays less attention to the analysis of whole structure and behaviors of web services. Recently, a number of Web Services-oriented Architecture Description Language (WSADL), such as Web Services Description Languages (WSDL) [10], Web Services Flow Language (WSFL) [108], Business Process Execution Language for Web Services (BPEL4WS) [1], Web Service Choreography Interface (WSCI) [9], XLANG [148], and others. have been developed and applied in both the academic and industry. However, these WSADLs either merely focus on static descriptions of web service functional activities as a whole, or concentrate only on the behavioral integration between web services. Furthermore, none of these current WSADLs support dynamic verification and monitoring of the integrated system.

Finally, web service design and composition is a distributed programming activity. It requires software engineering principles and technology support for service reuse, specialization and extension such as those used, for example, in component based software development. Although web service provides the possibility for offering new services by specialization and extension instead of designing them from scratch, to this date there is little research initiative in this context.

SAM [198] is an architectural description model based on Petri nets [153] well-suited for modeling distributed systems and temporal logic [137] for specifying system properties. In this section we propose a service-oriented architecture model, SO-SAM, which extends SAM to the web service applications and overcomes the drawbacks of current web service description languages. SO-SAM is an executable specification language based on 
the Predicate Transition Net (PrTN) [153] and the temporal logic [137] specification of system properties. Supporting modern software engineering philosophies equipped with component-based notations, SO-SAM provides an approach to specify and monitor the dynamic composition of web services and integration of service-oriented systems. SO-SAM model introduces the concept of component web to facilitate the idea of web service reuse, specialization, extension and online evolution.

\subsubsection{SO-SAM Specification}

In order to specify both the static and dynamic architectural features of a web servicesoriented system, we proposed a Service Oriented Software Architecture Model (SO-SAM) [74] as an executable architectural specification language. Two web service components are composed through connectors. SO-SAM specifies a web services-oriented software architecture as a set of connected architectural components described by PrT nets and service properties described by temporal logic formulae. The architectural components correspond to functional units in the system. With the hierarchical nature of SAM model one architectural component may in turn be composed of several smaller architectural components. The entire system can be viewed as a highest-level architectural composition, which is also a component. Each architectural component is either statically or dynamically realized by a web services component. Architectural components are connected to each other via XML message passing through Simple Object Access Protocol (SOAP) [7]. The behavior of the connection is specified by SAM architectural connectors. The message passing mediates the interactions between architectural components via the rules that regulate the component interactions. In the model, connectors carry the tasks of service compositions. Thus the model supports both executable and dynamic web service compositions.

Definition 12 (SO-SAM) Service-Oriented Software Architecture Model, SO-SAM, is defined by a set of components $C_{m}$, a set of connectors $C_{n}$, and a set of constraints $C_{s}$, e.g. $S O-S A M \triangleq<C_{m}, C_{n}, C_{s}>$, with following regulations: 
- Two components can communicate with each other only through connectors, which model service integration.

- The message in each port is defined in the XML format and passed through SOAP protocol.

- The component follows the definition of WSDL or XML.

- The dynamic behavior of a component is defined by a service net, which is focusing on its internal operational nature.

In Chapter 6, we know that the ports in each component are either input ports or output ports. In the extension to web applications, ports are used to transfer messages among services, same as in SAM model. However, we regulate messages as a tuple with the information of service name, service description, location, URL, etc., so that the message carries service information.

A component is composed of the above ports that carry service information, behavior description and property specification. The behavior of a component is defined by a Petri net, which is called a service net. In the service net, tokens in a place has to have specific sort to be consistent with the above port and message definition. A basic component is one that does not have sub-components and non-empty connectors, otherwise, it is a composition. A composition is a composite service. The relation between a composition and its subcomponents and connectors is defined by a mapping function $f$. Mapping function $f$ is a set of maplets from super component(connector)'s identities to sub-components'(connectors').

Service integration can be done through connectors. Connectors have the same definition as in SAM. The Petri net for a connector is a regular petri net that describe the integration and composition of services. A connector cannot be a composition.

Definition 13 (Port) Ports in SO-SAM are communication interfaces of each services and graphically represented by semi-circles. Messages in ports are modeled by tokens. The sort of each token is defined by two parts, service sort $S_{S}$ and data sort $S_{D}$. Service sorts are, but 
not limited to, SName, SDesc, Loc, URL, where SName is the name of the service, SDesc is the service description, Loc is the service location and URL is the Uniform Resource Locator. data sort is the regular sort defined in SAM [96].

Thus we have sort $S$ of a port is defined by

$$
S \triangleq S_{S}, S_{D}
$$

Service sort $S_{S}$ is a service query indicating a service name, location, description, URL, etc.. Service sort must be carried by all tokens in the service net.

Definition 14 (Service Component) Each component $C_{m}^{i}$ in $S O-S A M$ is defined by a tuple, component name $C_{m}^{i} I D$, mapping function $f$, set of ports $P_{t}$ that is composed of the set of input ports $P t_{I}$ and the set of output ports $P t_{O}$, the set of initial ports $P t_{\text {ini }} \in P_{t}$, the set of finial ports $P t_{f n l} \in P_{t}$, a service net $S N$, and a set of temporal logic formulae $S T$, e.g.,

$$
C_{m}^{i} \triangleq<C_{m}^{i} I D, f_{C_{m}^{i} I D}, P t_{i n i}, P t_{f n l}, P t_{\text {internal }},
$$

$S_{\text {des }}, S N, S T>$,

where $f_{C_{m}^{i} I D}$ is a mapping function : $C_{m}^{i} I D \rightarrow s u b C_{m}^{i} I D$, and $C_{m} I D$ and $s u b C_{m} I D$ denote the set of components and subcomponents respectively. If $f_{C_{m}^{i} I D}=\Phi$, then $C_{m}^{i}$ is a basic component and cannot be further refined. $P t_{i n i}, P t_{f n l}, P t_{\text {internal }}$ are set of initial, final and internal ports. $S_{\text {des }}$ is a service description.

Initial ports are represented by dash line bold half circles, and final ports are represented by solid line bold half circles. Each set of initial ports in a service component must connect to a set of final ports in another service component through a connector, and vice versa.

In the component, each service must be started from one set of initial ports, but can be ended at multiple finial ports separately. This is because a service can reach different final states but starts at the same condition.

Definition 15 (Service Net) $A$ service net is a Petri net defined by 8-tuple, $S N \triangleq<$ $P, T, F, \phi, R, L, M_{0}, \mathcal{M}_{p}>$, where

- $P, T$ are finite set of places and transitions; $F$ is flow relation: $P \times T \cup T \times P$. 
- $\phi$ is sort assignment: $P \rightarrow \wp(S)$ ([96]), but sorts $S$ are extended to carry service information.

- The final ports $P t_{f n l}$ of a service net communicate with a set of initial ports $P t_{i n i}$ of another service net through a connector.

- $R$ (guard function), L (label function), and $M_{0}$ (initial markings) follow the definitions in the paper [96].

- $\mathcal{M}_{p}$ is place mapping function, $\mathcal{M}_{p}: P_{t} \rightarrow P$

where each sort must contain $S_{S}$. Mapping function $\mathcal{M}_{p}$ associates each port to a place in the service net. It is a one-to-one mapping function, because in the SAM model the place and ports share the same name, and two ports between upper level and lower level share the same name. It is also an onto mapping function because all places that are identified as communicators to the other service nets will not be increased for a basic service component.

Definition 16 (Web Service) $A$ web service is defined by a basic component or $a$ composition (collection of components and connectors) or a tuple such that < $S_{I D}, f, S_{d e s}, P t, S N, S T>$ where

- $S_{I D}$ is the service which is equivalent to $C_{m}^{i} I D$. When a service component has more than one services, each service must contain its service component's ID.

- mapping function $f$ is equivalent to $f_{C_{m}^{i} I D}$ syntactically. The difference is the mapping function $f_{C_{m}^{i} I D}$ for service component should concern behavioral refinement, while in each service we only concern a flattened component where the ports that inherited from service component are the only mapping concern in $f$.

- $S_{\text {des }}$ is the service description which can be represented by WSDL,

- and $P t_{i n i} \cup P t_{f n l}=P t$. Since it is a basic service, not a composite service (i.e. service component) $P t_{\text {internal }}=\Phi$ 
- $S T$ is a subnet of $S T$ that is defined in the service component, and so is $S N$.

The behavior of each web service $S^{i}$ in SO-SAM is defined by a service net $S N$, which must starts when the initial ports $P t_{\text {ini }}$ has messages and ends when the final ports receive messages. The properties are defined using a set of temporal logic formulae ST.

The composition of different web services can be modeled by connectors.

\subsubsection{Service Composition}

Because services are simple units used for building distributed applications, we cannot talk about them without talking about service-based applications - specifically, how these applications are built based on the service view and how services should behave together within them based on the discovering and binding functions. The applications will use services by composing or putting them together. An architecture for service-based applications has three main parts: a provider, a consumer, and a registry. Providers publish or announce their services on registries, where consumers find and then invoke them. Figure 26 provides a general architectural model for Web services, which forms the basis for an SOA.

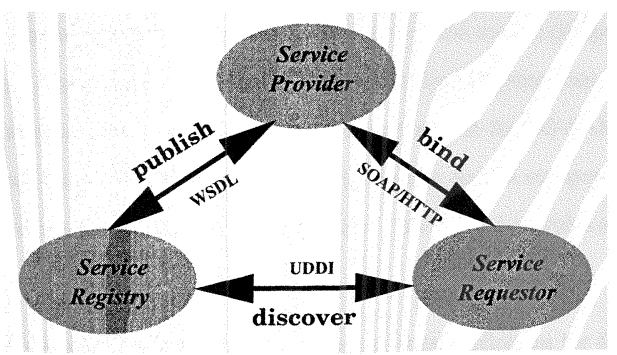

Figure 26: A General Service Oriented Architecture

In this section, we explain how the service component and connector work together to create service compositions. As shown in the Figure 26, there are three stages among the 
three participants of service composition: publishing the service descriptions, discovering the matching services, and binding the required services. We address these in the following.

The publishing stage involves service description, static syntax checking, and compatibility checking. The output of this stage is a service identified with its ID and service description.

Each service requested can be satisfied if we find the services that match the required constituent services either completely or partially. For service discovery, it is important to find an appropriate service with the right capability. Service discovery relies on the following steps:

- Functional analysis: this is to check the selected service's behavioral capability in terms of the functions they provide to determine whether or not this service can perform the completely or partially the requested service task.

- Semantic analysis: during this period, we compare the descriptions of the requested service with the retrieved services from the repository in terms of service contents and decide how closely they are related. High degree related service will be chosen. In this stage how to define the semantic of a service and service composition is quite difficulty. A service composition in a large distributed system can involve highly complicated sequential and parallel communications. In the SO-SAM model, each service can be described by a Petri net behaviorally and its composition is further distributed on a component-based architecture. This method provides a good solution for this issue.

- Syntactic analysis: Each service has a precise syntax definition for their interfaces and operations. In this stage we need to check the service syntax and determine how they can be combined to achieve the requested higher-order service.

We use $=$ to denote sequential composition, $\|$ denote parallel composition. Based on the SO-SAM model, we define the sequential and parallel composition as follows. 
Definition 17 Sequential Composition of Services can be defined as following:

A service $S$ is a sequential composition of two services $S 1$ and $S 2$, i.e., $S \triangleq S 1 \simeq S 2$, where

$$
\begin{aligned}
& S \triangleq<S_{I D}, f, S_{d e s}, P t, S N, S T> \\
& S 1 \triangleq<S_{I D_{1}}, f_{1}, S_{d e s_{1}}, P t_{1}, S N_{1}, S T_{1}>\text {, and } \\
& S 2 \triangleq<S_{I D_{2}}, f_{2}, S_{d e s_{2}}, P t_{1}, S N_{1}, S T_{1}>\text {, and }
\end{aligned}
$$

- $S_{I D} \triangleq S_{I D_{12}}$, the composed service ID is the combination of each service's ID,

- $f \triangleq f_{1}-f_{1_{f n l}} \cup f_{2}-f_{f_{f n l}}$, where $f_{1_{f n l}}$ and $f_{2 f n l}$ denote the $P t_{f n l_{1}}$ and $P t_{f n l_{2}}$ mapping in the $f_{1}$ and $f_{2}$ respectively,

- $S_{d e s} \triangleq S_{d e s_{1}} \cup S_{d e s_{2}}$,

- $P t \triangleq P t_{1} \cup P t_{2}$, where $P t_{i n i} \triangleq P t_{i n i_{1}}, P t_{f n l} \triangleq P t_{f n l_{2}}$, and $P t_{\text {internal }} \triangleq P t_{f n l_{1}} \cup P t_{i n i_{2}}$,

- $S N \triangleq S N_{1} \cup S N_{2} \cup\left\{p_{\text {wait }}\right\}$, where $p_{\text {wait }}$ is a connecting place between two sub-services $S_{1}$ and $S_{2}$,

- $S T$ cannot be defined as $S T_{1} \cup S T_{2}$ since they are not equivalent to each other.

Since sequential composition of two services $S_{1}$ and $S_{2}$ has an ordering relation, i.e., it could be $S_{1}$ concatenating with $S_{2}$ or vice versa.

It is worth to note that after the sequential composition the composed service $(S)$ property is not the simple union of each sub-service $\left(S_{1}, S_{2}\right)$ property because the atomic formula of each property is the initial and final ports while these ports in the new service is not the union of each sub-services $\left(S_{1}, S_{2}\right)$. Place $p_{\text {wait }}$ is necessary because each sub-service has to communicate with outside through their ports, the internal communication of the two services can be done by a place connecting two transitions in two sub-services to hold the information of the previous one and provide service data to its following one. Finally, the union of Petri nets can be defined as the merge of places with the same name. 
Definition 18 Parallel Composition of Services can be defined as following:

A service $S$ is a parallel composition of two services $S 1$ and $S 2$, i.e., $S \triangleq S 1 \| S 2$, where $S \triangleq<S_{I D}, f, S_{d e s}, P t, S N, S T>$

$S 1 \triangleq<S_{I D_{1}}, f_{1}, S_{d e s_{1}}, P t_{1}, S N_{1}, S T_{1}>$, and

$S 2 \triangleq<S_{I D_{2}}, f_{2}, S_{d e s_{2}}, P t_{1}, S N_{1}, S T_{1}>$, and

- $S_{I D} \triangleq S_{I D_{12}}$, the composed service ID is the combination of each service's ID,

- $f \triangleq f_{1} \cup f_{2}$, where $f_{\text {ini }} \triangleq f_{\text {ini }} \cup f_{\text {ini }}$, and $f_{f n l} \triangleq f_{f n l_{1}} \cup f_{f n l_{2}}$,

- $S_{d e s} \triangleq S_{d e s_{1}} \cup S_{d e s_{2}}$,

- $P t \triangleq P t_{1} \cup P t_{2}$, where $P t_{i n i} \triangleq P t_{i n i_{1}} \cup P t_{i n i_{2}}, P t_{f n l} \triangleq P t_{f n l_{1}} \cup P t_{f n l_{2}}$, and $P t_{\text {internal }} \triangleq \Phi$,

- $S N \triangleq S N_{1} \cup S N_{2} \cup P_{\text {comm }}$, where $P_{\text {comm }}$ is a set of communication places between two sub-services $S_{1}$ and $S_{2}$, if the two services have communication, otherwise, we have

$S N \triangleq S N_{1} \cup S N_{2}$

- It can be defined $S T \triangleq S T_{1} \cup S T_{2}$ if two sub-services do not have communication.

The basic services composition is described in one service component. In parallel, for the two service components, we can also define sequential composition.

Definition 19 Sequential Composition of Service Component can be defined as following:

A service component $S C$ is a sequential composition of two service components $S C 1$ and $S C 2, S C=S C 1=S C 2$, where

$$
S C \triangleq<C_{I D}, f_{C}, P t_{i n i}, P t_{f n l}, P t_{\text {internal }},
$$

$S_{d e s}, S N, S T>$,

$S C 1 \triangleq<C_{I D 1}, f_{C} 1, P t_{i n i_{1}}, P t_{f n l_{1}}, P t_{\text {internal }}$,

$S_{d e s_{1}}, S N_{1}, S T_{1}>$, and

$S C 2 \triangleq<C_{I D 2}, f_{C} 2, P t_{i n i_{2}}, P t_{f n l_{2}}, P t_{i_{n t e r n a l}}$,

$S_{d e s_{2}}, S N_{2}, S T_{2}>$, and 
- $C_{I D} \triangleq C_{I D_{12}}$, the composed service component/composition ID is the combination of each service component's ID,

- $f_{C} \triangleq f_{C 1}-f_{C 1_{f n l}} \cup f_{C 2}-f_{C 2 \text { fnl }}$, where $f_{C_{\text {ini }}} \triangleq f_{C_{i n i_{1}}}, f_{C_{f n l}} \triangleq f_{C_{f n l_{1}}}$, and $f_{C_{\text {intermal }}} \triangleq$ $f_{C_{f^{\prime} l_{1}}} \cup f_{C_{\text {ini }}} \cup f_{C_{\text {intermal }_{1}}} \cup f_{C_{\text {internal }}}$

- $S_{d e s} \triangleq S_{d e s_{1}} \cup S_{d e s_{2}}$,

- $P t \triangleq P t_{1} \cup P t_{2}-P t_{f n l_{1}}-P t_{i n i_{2}}$, where $P t_{\text {ini }} \triangleq P t_{i n i_{1}}, P t_{f n l} \triangleq P t_{f n l_{2}}$, and $P t_{\text {internal }} \triangleq$ $P t_{f_{n} l_{1}} \cup P t_{\text {ini }_{2}} \cup P t_{\text {internal }_{1}} \cup P t_{\text {internal }_{2}}$,

- $S N \triangleq S N_{1} \cup S N_{2} \cup N_{C n}$, where $S N_{C n_{12}}$ represents the Petri nets of the connector $C n_{12}$ between service components $S C 1$ and $S C 2$

- $S T$ cannot be defined as $S T_{1} \cup S T_{2}$ since they are not equivalent to each other.

It is worth to note that there is not a necessary ordering relation between two service components. When we denote the sequential composition of two service components, we need to consider the connector between them, i.e., $S C \triangleq S C 1=S C 2 \equiv S C 1=C n_{12}=$ $S C 2$.

\subsubsection{A Case Study on the SO-SAM Model}

The top topology of an online shopping system specified in SOSAM model is given in Fig. 24. Each description block is captured in a component (or a composition). Each composition/component captures a service with the start and end ports. For instance, service Customer and ShoppingCart is modeled in the component Customer and component Cart, and both components form a composition, a composite service, User in the topology of SOSAM. In the composition User, a customer starts from request to browse the web (through port request_51), then choose category, select and checkout items. Finally it ends at either getting the order and ship information (port ordNum_15 and shipEmail_5) or payment errors (port chkoutErr_5). In addition, a user can also adjust the order and cart list. All internal operations are represented by the refined inner components, service nets and connectors. 
Description blocks Warehouse and OrderProcessing are specified in the service component Warehouse and Order, which form another composite service E-Company. An E-company starts at port request $\_27$ to evaluate user requests, and confirm or reject user requests, then submit user information forms and generate orders, etc.. Finally, the service component PaymentProcess captures the behavior of the description block CreditCardProcessing. Description block RetailerBank is simply represented in the service component RetailerBank.

Since SO-SAM model keep the port same as that in the SAM model, the properties specified for the online shopping example are still valid in this SO-SAM model. The interesting aspect about this case study is that we can formally verify and validate service composition through the SO-SAM model. Furthermore, the SO-SAM model provide an integration among services.

\subsection{Application on UML Architecture Description of Web Systems}

Web systems are self-descriptive software components which can automatically be discovered and engaged, together with other web components, to complete tasks over the Internet. The importance of web system architecture descriptions has been widely recognized in recently year. One of the main perceived benefits of a web system architecture description is that such a description facilitates system property analysis and thus can detect and prevent web design errors in an earlier stage, which are critical for web systems. Software architecture description and modeling of a web system plays a key role in providing the high level perspective, triggering the right refinement to the implementation, controlling the quality of services of products and offering large and general system properties. While several established and emerging standards bodies (e.g., $[1,2,7,9,10]$ etc.) are rapidly laying out the foundations that the industry will be built upon, there are many research challenges behind web system architecture description languages that are less well-defined and understood [107] for the large number of web service application design and development. 
On the other hand, Unified Modeling Language (UML), a widely accepted objectoriented system modeling and design language, has been adapted for software architecture descriptions in recent years. Several research groups have used UML extension to describe the web system's architecture $([29,100])$. However, it is hard to detect the system problems, such as correctness, consistency [101] and others, of the integration of Web services without a formal semantics of web services architecture.

Currently, although a software architecture description using UML extension contains multiple viewpoints such as those proposed in the SEI model [164], the ANSI/IEEE P1471 standard, and the Siemens [103]. The component and connector (C\&C) viewpoint [187], which addresses the dynamic system behavioral aspect, is essential and necessary for system property analysis.

To bridge the gap between web system architecture research and practice, several researchers explored the ideas of integrating architecture description languages (ADLs) and UML $[40,61,62,140]$. Most of these integration approaches attempted to describe elements of ADLs in terms of UML such that software architectures described in ADLs can be easily translated to extensions of UML. There are several problems of the above approach that hinder their adoption. First, there are multiple ways to describe ADLs in terms of UML [79], each of which has advantages and disadvantages; thus the decision on which extension of UML to use is not unique. Second, modifications on UML models are difficult to be reflected in the original ADL models since the reverse mapping is in general impossible. Finally, the software developers are required to learn and use specific ADL to model software architecture and use the specific extension of UML, which is exactly the major cause of preventing the wide use of ADLs. Currently, there is less work involved to apply these methodologies to the web systems.

In this section, we present an approach opposite to the one mentioned above and apply this approach to the web applications, i.e. we translate a UML architecture description into a formal architecture model for formal analysis. Using this approach, we can combine the potential benefits of UML's easy comprehensibility and applicability with a formal 
ADL's analyzability. Moreover, this approach is used to formally analyze the integration of web services. The formal architecture model used in this research is named SO-SAM, an extended version of SAM [97], which is based on Petri nets and temporal logic; and supports the analysis of a variety of functional and non-functional properties [99]. Finally, we validate this approach by using model checking techniques.

\section{Algebraic High-Level Nets}

An algebraic high-level net integrates Petri net with inscription of an algebraic specification defining the data types and operations. Instead of specifying a single system model, an algebraic Petri net represents a class of models that often differ only in a few parameters. Such a compact parameterized description is unavoidable for modular specification and economic verification of net models in the dependable system design.

Generally speaking, an algebraic high-level (AHL) nets $N=$ ( $S P E C, A, X, P, T, W^{+}, W^{-}$, cond, type) consists of following parts:

- An algebraic specification $S P E C=(S, O P, E)$, where $S I G=(S, O P)$ is a signature, and $\mathrm{E}$ is a set of equations over $S I G$;

- $A$ is an $S P E C$ algebra;

- $X$ is an family of S-sorted variables;

- $P$ is a set of places;

- $T$ is a set of transitionssuch that $P \cap T=\emptyset$;

- Two functions $W^{+}, W^{-}$assigning to each $t \in T$ an element of the free commutative monoid ${ }^{1}$ over the cartesian of $P$ and terms of $S P E C$ with variables in $X$.

- cond is a function assigning to each $t \in T$ a finite set of equations over signature SIG.

\footnotetext{
${ }^{1} \mathrm{~A}$ set $\mathrm{M}$ with an associative operation * and an identity element for that operation is called a monoid. A commutative monoid is a monoid in which the operation is commutative. A commutative monoid is a free commutative monoid if every element of $\mathrm{M}$ can be written in one and only one way as a product (in the sense of *) of elements of subset P M.
} 
- type is a function assigning to each place a sort in $S$.

\section{Component and Connector View}

Component and connector view was one of the four views proposed in $[103,104]$, which is described as an extension of UML. The component and connector view describes architecture in terms of application domain elements. In this view, "the functionality of the system is mapped to architecture elements called components, with coordination and data exchange handled by elements called connectors." [103]

In the component and connector view, components, connectors, ports, roles and protocols are modelled as UML stereotyped classes. Each of them is represented by a special type of graphical symbol, as summarized in Fig. 27. A component communicates with another component of the same level only through a connector by connections, which connect relevant ports of components and roles of connectors that obey a compatible protocol. In addition to the connections between components and connectors, ports (roles, resp.) of a component (connector, resp.) can be bound to the ports (roles, resp.) of the enclosing component (connector, resp.).

\subsubsection{An Image Processing Example}

In order to present the approach we use an image processing example used in the distributed web application that was adapted from [103]. Fig. 28, 29, 30 from [103] shows a concrete and complete component and connector view, which is the running example of this section. Fig. 28(a) is a configuration of ImageProcessing component. Fig. 28(b) shows another aspect of the configuration. Both of them are UML class diagrams and model different aspects of the system. From these two figures, we can see the component ImageProcessing contains two components: Packetizer and ImagePipline, and one connector PacketPipe. The ports of component ImageProcessing, rawDataInput, acqControl, and framedOutput are bound to ports rawDataInput of component Packetizer, acqControl and framedOutput of component ImagePipeline respectively. Component Packetizer communicates with component ImagePipeline through connector PacketPipe. Component 


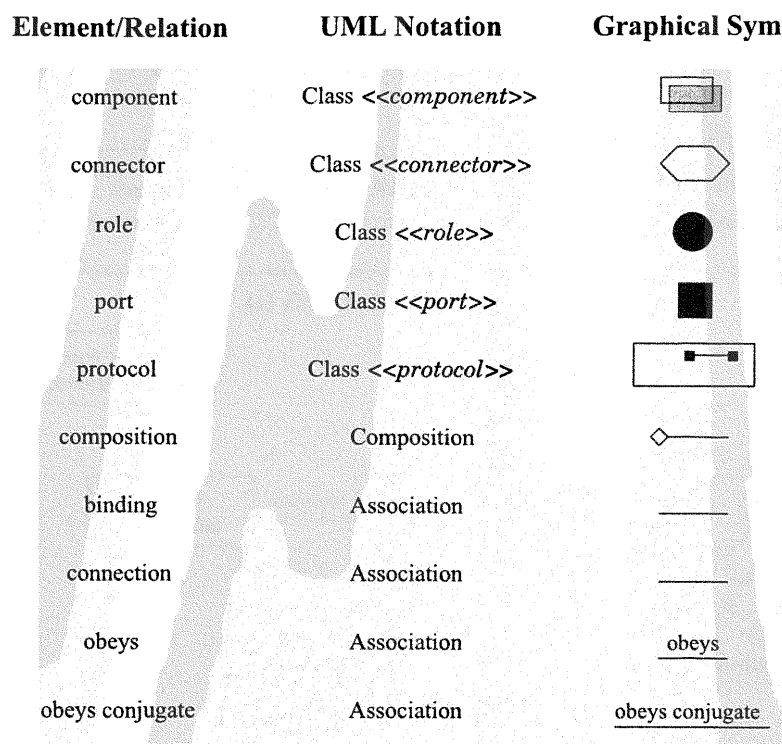

Figure 27: UML Extension for component and connector View

Packetizer and connector PacketPipe is connected by a connection between port PacketOut and role source, which obey (conjugate) protocol DataPacket. Component ImagePipeline and connector PacketPipe is connected by a connection between port PacketIn and role dest, which obey (conjugate) protocol RequestDataPacket. Being a conjugate means that the ordering of the messages is reversed so that incoming messages are now outgoing and vice versa.

Fig. 28 alone is not enough to illustrate component and connector view since only components and connectors of the system and corresponding connections among them are demonstrated. Additional diagrams are needed to define protocols and functional behavior of components and connectors. A protocol, represented by a stereotyped class, is defined as a set of incoming message types, a set of outgoing message types and the valid message exchange sequences. The valid message exchange sequence is represented by a sequence diagram. Fig. 29 shows the definition of RawData, DataPacket, and RequestDataPacket 


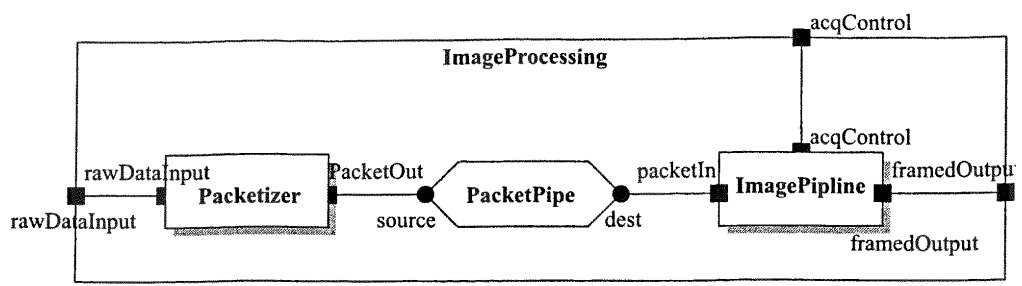

(a) Component ImageProcessing

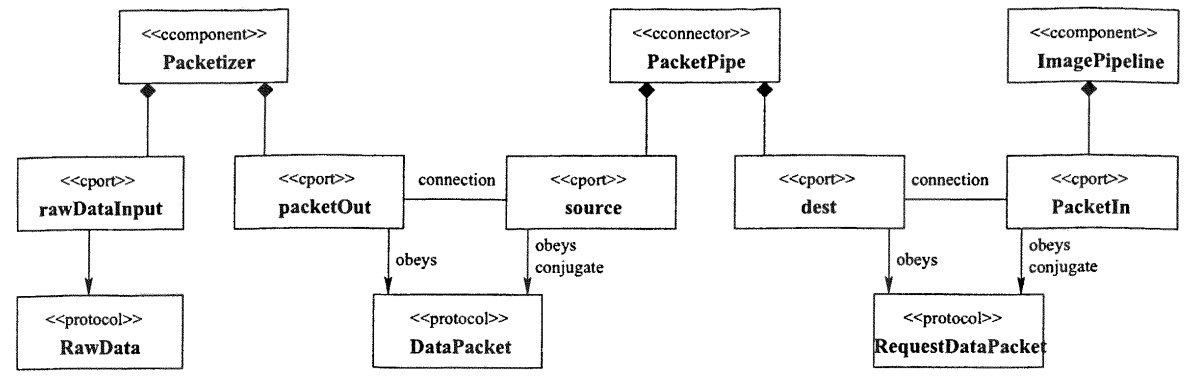

(b) Protocols for Packetizer and PacketPipe

Figure 28: Structural Aspect of Component and Connector View

protocols. From Fig. 29(c), we can see protocol RequestDataPacket has one incoming message: packet(pd), and three outgoing messages: subscribe(c), desubscribe(c), and requestPacket(c) where $c, p d$ are parameters of messages. In order to communicate with object $B$ based on protocol RequestDataPacket, object $A$ first sends object $B$ a message subscribe(c) where $c$ indicates the sender $A$. Then a message requestPacket is sent to $B$ to request a packet. Later, object $A$ may receive a packet $p d$ from $B$. The symbol "*” in the figure indicates that the pair of message requestPacket and packet(pd) may occur many times. Finally, object $A$ sends a message desubscribe(c) to $B$ to stop requesting packet.

The behavior of components/connectors may be described formally by UML statechart diagrams, for example the behavior of component Packetizer and connector PacketPipe in Fig. 30. Statechart diagrams describe the dynamic behaviors of objects of individual classes through a set of conditions, called states, a set of transitions that are triggered by event instances, and a set of actions that can be performed either in the states or during 


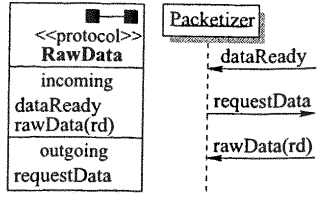

(a) Protocol RawData

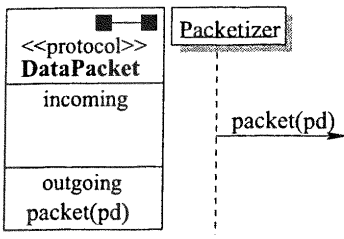

(b) Protocol DataPacket

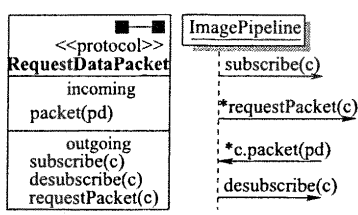

(c) Protocol RequestDataPacket

Figure 29: Protocols in Component ImageProcessing

the firing of transitions. From Fig. 30(b), we can see the statechart diagram of connector PacketPipe contains two states: waiting and "assign packet to ready client" and seven transitions. When connector PacketPipe receives an event subscribe(c), it invokes its operation AddClient(c) although we do not know exactly the functionality of this operation. When the connector receives an event $\operatorname{packet}(p d)$, it saves the packet $p d$. And the response of connector PacketPipe to an event requestPacket(c) is up to the condition: the client $c$ has read current packet or not. If yes, the connector treats it as a request for next packet; otherwise it sends current packet to client $c$ through an event $c$.packet( $p d)$. If all clients have read current packets, the connector updates its packet queue and enter state "assign packet to ready client" in which the connector sends current packet to clients that has submitted their requests. If all requests are processed, the connector return to state waiting. As we can see from this figure, connectors and components mainly handle incoming messages of protocols they obey (conjugate).

\subsubsection{Transformation From Component \& Connector View}

Component and connector (C\&C) view [187] has been the main underlying design principle of most ADLs [142], which is also a major view type in several software architecture documentation models supporting multiple architecture views such as SEI [164] and Siemens [103]. C\&C view is essential and necessary for system dependability analysis since it captures a system's dynamic behavioral aspect. SO-SAM model and component and connector view share a set of common terms such as components, connectors, and 


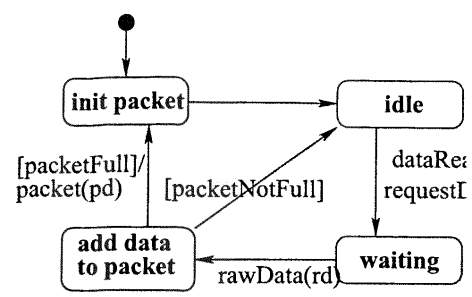

(a) Behavior of Component Packetizer

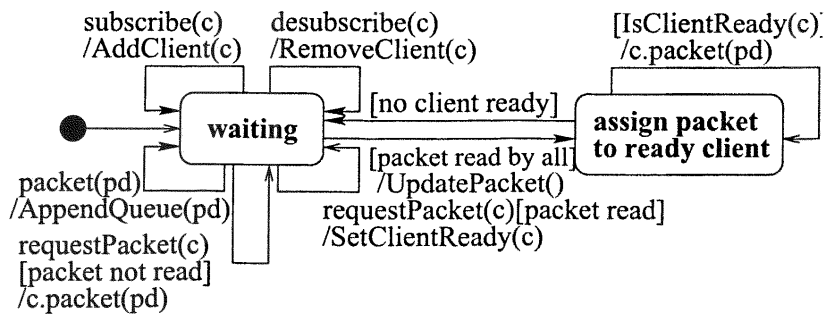

(b) Behavior of Connector PacketPipe

Figure 30: Behavior of Elements (UML)

ports. Therefore it is straightforward to map them to the counterparts in SO-SAM. However, due to the meaning difference and various formal methods to describe elements' behavior, the concrete mapping procedure is not that easy. This section shows a method [75] to construct a complete and executable SO-SAM model from a component and connector view.

A component (connector, resp.) in component and connector view is mapped to a component (connector, resp.) in SO-SAM model. It is easy to understand from structural aspects. However, the behavior mapping is complex since different formal methods are used to model behavior. UML statechart diagrams are used to model behavior in component and connector view, contrasting with Petri net model in SO-SAM. Fortunately, the previous work [55] showed that it is possible to transform statechart diagrams to Petri net models. In UML statechart diagrams, method invocations and relationships between variables are implicit in the elements' structure. For example, in Fig. 30(a), the conditions PacketNotFull and PacketFull, and relationship between variable $r d$ and $p d$ is not illustrated explicitly. 
However, such information has to be expressed explicitly in order to obtain a complete and executable Petri net. In order to bridge the gap, we utilize algebraic high level nets [65], a variant of high level Petri nets, to model behavior of elements. This method is possible because SO-SAM model does not specify a particular Petri net model as its formal foundation. We use algebraic specifications [63] to capture structures of elements obtained from UML statechart diagrams because algebraic specifications are abstract enough that no additional information about implementation detail is assumed, and they are also powerful enough to represent implied information about components or connectors. Although the work [55] is for SAM architecture model, we can still use it and adapt it to the SO-SAM model since they share the same net structure. The main differences exist in the service sorts in the net specification, initial and final ports in the net specification and net inscription. The following rule gives us a general idea to derive components or connectors in SO-SAM.

Rule 1 (Component and Connector) A Component (connector, resp.) in component and connector view is mapped to a component (connector, resp.) in SO-SAM according to following steps:

Step 1 An algebraic specification, which specifies the abstract interface of the component (connector, resp.), is generated from a UML statechart diagram. The idea to construct algebraic specification is described later.

Step 2 Construct a complete and executable algebraic high level net from the UML statechart diagram according to the approach in [55] and the generated algebraic specification. There is a special place in the generated algebraic high level nets that contains element information and provides necessary information for transitions.

Step 3 A component (connector, resp.) with a UML statechart diagram in component and connector view is mapped to a component (connector, resp.) with an algebraic high level net in SO-SAM. 
While it is inherently impossible to prove the correctness of the transformation, we have carefully validated the completeness and consistency of our transformation rule. First, from structure point of view, concepts of components or connectors in component and connector view and SO-SAM are the same. Both of them support composition, binding with enclosing element, and they can have their own behavior and communication channels-ports or roles. Therefore, the main functionalities of components or connectors in component and connector view are presented in SO-SAM counterparts. Second, algebraic specification can be used to specify modular, more specific classes [64]. Therefore, the implied information of statechart diagrams, i.e. the operations and their properties can be correct and fully specified by algebraic specifications. Since functions of algebraic specifications only define what to be done, no additional implementation information not implied in statechart diagrams is introduced. Finally, our previous work [55] and others work [180] have shown that the behavior described by statechart diagrams can be fully captured by corresponding Petri nets.

The idea to obtain algebraic specifications from UML statechart diagrams is as follows: For each element (component and connector), its algebraic specification defines a sort, called element_sort, like packetizer for component Packetizer and packetpipe for connector PacketPipe. If a data type of parameters is not defined by a primitive algebraic specification, a new algebraic specification is imported. Such a imported algebraic specification generally defines only one sort, like Packet for component Packetizer. Each action of transitions in a UML statechart diagram is considered as a function such that: action_name $:$ element_sort $\times$ parameters_sort_list $\rightarrow$ element_sort. Here parameters_sort_list includes service sorts as well. For a guard condition of a transition, a function from elementsort (with necessary parameter sorts) to boolean is added. For each variable that is defined in the element (i.e. the variable is not defined in the related events), a function like GetVariableTypeName $:$ element_sort $\rightarrow$ element_sort $\times$ VariableType ${ }^{2}$

\footnotetext{
${ }^{2}$ This is actually the abbreviation of two functions: GetVariable : element_sort $\rightarrow$ VariableTypes and UpdateElement : element_sort $\rightarrow$ element_sort since these two functions are invoked sequently in the example.
} 
is specified. The properties of these functions can be constructed as equations if they are implied in the UML statechart diagrams, like guards PacketNotFull and PacketFull cannot hold at the same time.

From the above idea, we know that the algebraic specification for component Packetizer contains four functions, two of them correspond to guard conditions: .PacketNotFull(), ..PacketFull() : packetizer $\rightarrow$ bool; and one is obtained from actions:_AddRawData(_) : packetizer $\times$ rawdata $\rightarrow$ packetizer, and one from undefined variable: _.GetPacket() : packetizer $\rightarrow$ packetizer $\times$ packet. In these functions, “_” is used to indicate a variable placeholder, bool is a sort defined in primitive algebraic specification Bool [63], and sorts rawdata and packet are defined in imported algebraic specifications Packet and RawData respectively, which are defined by users and normally only one sort (rawdata, packet resp.) is specified.

With these algebra specifications, we can generate corresponding algebraic high level nets according to Rule 1. Fig. 31 shows the generated Petri nets from UML statechart diagrams in Fig. 30. Each generated Petri net has three special places: RECV containing messages from environment, SEND temporarily storing messages generated for its environment, and the place whose name is the same as its element's name (here, Packetizer and PacketPipe resp.), holding the abstract structural information of the element. In additional to these three places, there is a corresponding place indicating current status for each state in statechart diagrams, for example, places idle,waiting, init_packet and add_data for the same name states. A special token in these places indicates if the corresponding state is active. Place RECV sends events from external environment to places that are interested in the event. In Fig. 31(a) place idle and waiting are interested in event dataReady and rawdata(rd) respectively. If state idle is active and an event dataReady is available, transition $t_{15}^{2}$ is fired. As a result, an event requestData is added to place SEND, and place waiting becomes active. State add_data becomes active if state waiting is active and an event rawdata $(r d)$ is available. At the same time, the token in place packetizer is changed to another one through operation _.AddRawData(-) of algebraic specification Packetizer. 
Components and connectors in component and connector view are connected through a connection if they are enclosed directly by the same element and the corresponding ports and roles obey (conjugate) a compatible protocol. Therefore, the mapping from ports or roles in component and connector view to ports in SO-SAM is actually the mapping from relevant protocols describing behavior of ports or roles to ports of SO-SAM components/connectors. However, ports in SO-SAM models have their own characteristics. A port in SO-SAM model is a place that has either no incoming arcs or no outgoing arcs. In other words, the communication between ports is unidirectional. Therefore, a protocol in component and connector view, which consists of a set of incoming message types, a set of outgoing message types and the valid message exchange sequences, is mapped into a set of interface places (To avoid confusion, we use interface places to refer to ports in SO-SAM model). The type of tokens in an interface place is OID $\times O I D \times M E S S A G E \_T Y P E$, where $O I D$ is a set of unique identification number for each instance of the element, which specifies sender and receiver of a message, and MESSAGE_TYPE is the set of message types of the protocol (Here we ignore the parameters of messages for brief). Rule 2 specifies how to map a port/role in component and connector view to interface places in SO-SAM.

Rule 2 (Ports and Roles) A port (role, resp.) of a component (connector, resp.) in component and connector view is mapped to a set of interface places of the corresponding component (connector, resp.) specified by Rule 1: For each protocol that the port (role, resp.) obeys (conjugate), each kind of incoming messages is mapped to an incoming (outgoing, resp.) interface place of the component (connector, resp.) with the name of the message type; and each kind of outgoing messages is mapped to an outgoing (incoming resp.) interface place of the component (connector, resp.).

Initial and final ports can not be obtained from the UML architecture description directly. We provided two possible solutions:

- One is extending $C \mathcal{G} C$ view with new UML stereotypes initialPort and finalPort. 
This would bring a direct transformation from CEC architecture to SO-SAM. The problem is this also bring more complexity into UML architecture description.

- Another is manually adding the specification for these ports according to the system architecture description. For instance, we can say dataReady and RawData as initial port and frameout and final port in this case.

A port (role, resp.) of an element is actually -roughly speaking- a "channel" that forwards messages of specified types either from element itself to environment, or from environment to element. In Rule 2, a token represents an occurrence of an message of specified type, and the direction of a message is specified by the place containing the token - incoming or outgoing. Therefore, the mapping in Rule 2 conserves the main structural features of ports/roles and related protocols, and the reverse mapping exists, which ensures the correctness of the rule.

The behavior of a protocol, defined by UML sequence diagrams to demonstrate valid message exchange sequences, actually specifies possible sequences of relevant messages along time axle. A sequence of protocol messages illustrates their occurrence order, which can be specified by a set of temporal constraints, the basic predicates of which are the names of interface places obtained through Rule 2. For example, from Rule 2, we know port RawData of Packetizer is represented by two incoming places dataReady and rawData, and one outgoing place requestData. We use predicate dataReady $\left(\left\langle s i d\right.\right.$, rid, $\left.\left.m_{\text {dataReady }}\right\rangle\right)$ to describe if place dataReady contains a token representing an event dataReady that is sent to rid by sid.

In order to construct temporal constraints, we consider two elements communicating with each other through a protocol, for example RawData. First we only consider a pair of adjacent events, for example DataReady and requestData. For this pair of events, it means if an event DataReady occurs, then an event requestData must occur some time later, which is described by a temporal formula:

$$
\forall<\text { sid, rid, } m_{d}>, \square\left(\text { dataReady }\left(<s i d, \text { rid }, m_{d} \gg\right) \rightarrow \diamond \text { requestData }\left(<s i d, \text { rid, } m_{>}>\right)\right)
$$


However, this temporal formula cannot reflect the situation implied in the sequence diagram of the protocol: no other events of the protocol can occur between events dataReady and requestData. In order to describe this implied property, we have a reasonable assumption at architecture level that the communication media is reliable, no message is lost and no need to resend a message. Therefore, another temporal formula is introduced to address this missing situation:

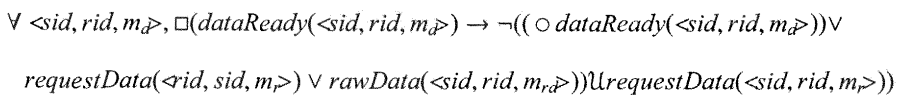

This formula is a safety property in first order temporal logic. This temporal formula means if an event of dataReady occurs, no other events such as dataReady, requestData and rawData can occur before the first event of requestData. Predicate odataReady $(<$ sid, rid, $m_{d}>$ ) is used to guarantee that the temporal formula is satisfied at the time the event dataReady occurs. Therefore, given a sequence diagram of a protocol with $n$ messages, we can obtain $(n-1) * 2$ temporal formulas.

In addition to the consideration of one session of a protocol, we have to inspect the relationship of two adjacent sessions of the same protocol between two objects, i.e. one session can start only after the previous session ends. Such a relationship is specified by a temporal constraint:

$$
\begin{aligned}
& \left.\forall<\text { sid, rid, } m_{r d}\right\rangle, \square\left(\text { rawData }\left(<\text { sid, rid }, m_{r \ngtr}\right\rangle\right) \rightarrow \neg\left(\text { dataReady }\left(\left\langle\text { sid }, \text { rid }, m_{d}\right\rangle\right) \vee\right. \\
& \text { requestData } \left.\left(\triangleleft i d, \text { sid, } m_{p}>\right) \vee\left(\text { o rawData }\left(<\text { sid, rid, } m_{r \triangleright} \gg\right)\right)\right) \text { UdataReady }\left(\left\langle s i d, \text { rid, } m_{d} \gg\right)\right)
\end{aligned}
$$

Although we think the above generated constraints are strong enough, there is still one more case we ignored: the first session of a protocol in a running system may starts with any messages but the first message. For example, a session of protocol RawData starts with message dataReady, and then obeys relevant part of the sequence diagram. We can 
see this session satisfies the above temporal formulas, but conflicts with the behavior of the protocol. Such a case can be avoided in three different ways, and the choice of them is up to users. One is to introduce a temporal predicate basetime that holds only at the time "zero", and a new temporal formula:

$$
\begin{gathered}
\square\left(\text { basetime } \rightarrow \neg \left(\text { dataReady }\left(<\text { sid, rid, } m_{d} \gg\right) \vee \text { requestData }\left(\varangle \text { rid }, \text { sid, } m_{r}>\right) \vee\right.\right. \\
\text { rawData } \left.\left(<\text { sid }, \text { rid }, m_{r d} \gg\right)\right) \text { UdataReady }\left(\left\langle\text { sid, rid, } m_{d} \gg\right)\right)
\end{gathered}
$$

The second method is to introduce a past time operator such as "eventually in the past". The final way is to prove that system structure guarantees that such case cannot happen. Thus, from the above discussion, a sequence diagram for a protocol is mapped to a set of temporal constraints.

The following rule is used to construct a set of constraints for components or connectors according to the above discussion.

Rule 3 (Constraint) For each protocol that a port (role, resp.) obeys (conjugate), a set of constraints, generated from the corresponding sequence diagram according to the above discussion, is added to the property specification of corresponding components (connectors, resp.) . When a constraint is added to a component (connector, resp.), sid or rid in tokens (the choice is up to the direction of corresponding message) is substituted by the actual identification number of the component (connector, resp.) since the component (connector, resp.) can only receive messages sent to itself.

A sequence diagram of a protocol specifies possible message communication sequences. However, it is impossible to limit the firing sequences of transitions in Petri nets to meet specified occurrence sequences of tokens in places. Although we cannot specify the firing sequences of transitions, but we can prove that if each possible firing sequence meets the behavior of a protocol. From the above discussion, we can see the generated set of temporal formulas exactly realizes the behavior of a protocol - the message sequences. 
By adding these temporal formulas as property specifications to components/connectors obeying the protocol, inconsistencies between behavior of elements and protocols can be easily detected. Since the behavior mapping in Rule 1 is complete and consistent, we know the detected inconsistencies also exist in the original model, i.e. Rule 3 is complete and consistent.

We may obtain a component (connector, resp.) with a behavioral model, and related ports and constraints according to Rules 1,2 and 3 respectively. Next task is to get a complete component or connector, i.e. ports of a component or connector has to be integrated with its behavior model. Rule 4 is used to guide such a procedure, and Rule 5 establishes the connection between components and connectors.

Rule 4 (Integration) The interface places, i.e. ports of a component (connector, resp.) in SO-SAM are integrated into its behavior model with the previous generated algebraic high level nets according to the following steps:

Step 1 Each incoming interface place is connected to place RECV through a transition, firing of which transmits tokens in the incoming place that are sent to the instance of component or connector to place RECV unconditionally.

Step 2 Each outgoing interface place is connected to place SEND through a transition, which forwards tokens of a special type in place SEND to the outgoing place.

Rule 5 (Connection) From Rules 2 and 4, if there is a connection between ports of a component and a role of a connector, then generated behavior models of the component and connector share a set of places that corresponds to the protocol they obey (conjugate). Therefore, to establish the connection between a component and a connector in SO-SAM, we merge these shared interface places because an incoming (outgoing, resp.) interface place in the component has an outgoing (incoming, resp.) counterpart in the connector such that they contain messages of the same type, and vice versa. 
In component and connector view, relationships between ports and behaviors are not specified explicitly. A port forwards incoming messages to the queue of the component/connector, which provide events for its behavior - the statechart diagram. The statechart diagram sends messages to its environment through a port. In Rule 4, place SEND serves as output queue and place RECV is input queue. The forward action is represented by the firing of transitions connecting place SEND, RECV and other interface places. Therefore Rule 4 captures the communication between ports and the corresponding behaviors in component and connector view.

Due to the space limitation, we cannot specify the transformation of binding and multiplicity. However, such transformations are similar and straightforward. Fig. 32 shows the final result of generated SO-SAM model from the running example. In order to give a concise description, algebraic specifications and internal parts of behavioral models are omitted.

In Fig. 32, components, for example component Packetizer and component ImageProcessing are represented by solid rectangles, while connectors such as PacketPipe are represented by dashed rectangles. The Petri nets enclosed by rectangles are the behavior models of components or connectors. Semicircles on the edge of rectangles are places that represent ports derived from relevant protocols. An inside semicircle indicates an incoming place that only receives tokens from environment, while an outside semicircle indicates an outgoing place that only sends tokens to environment. For example, component Packetizer has two incoming places dataReady, rawData, and one outgoing place requestData. These three places are derived from protocol RawData according to Rule 2. Component Packetizer and connector PacketPipe is connected through Rule 5. The binding between components and its enclosing component is implemented as a transition between corresponding places, which only forwards tokens from one place to another according to types of places (i.e. incoming or outgoing). For example, transition $t_{11}^{1}$ forwards tokens in place dataReady of ImageProcessing to place dataReady of Packetizer, while transition $t_{13}^{1}$ forwards tokens in place requestData of Packetizer to place requestData of ImageProcessing. 
Finally, we give an execution path of component ImageProcessing. Let component Packetizer be in state idle, connector PacketPipe in state waiting, and place ImageProcessing.dataReady contains a token representing message dataReady. This initial condition can be represented by the initial marking (ImageProcessing.dataReady, Packetizer.idle, PacketPipe.waiting). Here we only list related places (not including places such as packetizer and PacketPipe) that contain tokens, and ignore concrete token values that can be derived from context. We also assume that a packet consists of only one raw data, i.e. operation PacketFull() will be true if AddRawData() is invoked once. Table 18 shows the execution of communication based on protocols RawData and DataPacket. This example demonstrates the application of this method.

\subsubsection{Validation of the Approach}

The SO-SAM model allows formal validation of a service net against system constraints and property specified on its abstraction represented by a component or connector. Here, validation means that the developer can animate the specification by providing initial markings and checking if the responses meet the expected results. Validation of SO-SAM is based on the precise syntax and semantics of Petri net formal language and temporal logic. The validation will cover the topology and dynamic behavior of the Petri net as well as temporal logic formulae. Here we simply introduce how to translate SO-SAM model to the Maude [47] language. For the details, please refer to the work [77].

\section{Translation from SO-SAM to Maude}

Step 1. Translation to the functional module: generate the sorts operators used in the functional modules for the model signatures. This step translates each place, sorts, markings in a Petri net into the corresponding part in Maude's functional module.

Step 2. Translation to the system modules: there are three types of system modules, one is for the model signature that corresponds to the architecture structure and dynamic behavior of the model, one is for the mapping to the predicates, and one is for the model checking, which includes the property specification. 
Table 18: A Path of Executing Protocols RawData and DataPacket

\begin{tabular}{|c|c|c|}
\hline Step & Marking of Component ImageProcessing & Fired Transition \\
\hline 1 & idle, ImageProcessing.dataReady, PacketPipe.waiting & $t_{11}^{1}$ \\
\hline 2 & idle, Packetizer.dataReady, PacketPipe.waiting & $t_{19}^{2}$ \\
\hline 3 & idle, Packetizer.RECV, PacketPipe.waiting & $t_{12}^{2}$ \\
\hline 4 & idle, PacketPipe.waiting & $t_{15}^{2}$ \\
\hline 5 & Packetizer.waiting, Packetize.SEND, PacketPipe.waiting & $t_{111}^{2}$ \\
\hline 6 & Packetizer.waiting, Packetizer.requestData, PacketPipe.waiting & $t_{13}^{1}$ \\
\hline 7 & Packetizer.waiting, ImageProcessing.requestData, PacketPipe.waiting & unspecified transition \\
\hline 8 & Packetizer.waiting, PacketPipe.waiting & unspecified transition \\
\hline 9 & Packetizer.waiting, ImageProcessing.rawData, PacketPipe.waiting & $t_{12}^{1}$ \\
\hline 10 & Packetizer.waiting, Packetizer.rawData, PacketPipe.waiting & $t_{110}^{2}$ \\
\hline 11 & Packetizer.waiting, Packetizer.RECV, PacketPipe.waiting & $t_{13}^{2}$ \\
\hline 12 & Packetizer.waiting, PacketPipe.waiting & $t_{16}^{2}$ \\
\hline 13 & Packetizer.add_data, PacketPipe.waiting & $t_{17}^{2}$ \\
\hline 14 & Packetizer.initial_packet, Packetizer.SEND, PacketPipe.waiting, & $t_{18}^{2}$ \\
\hline 15 & Packetizer.idle, Packetizer.SEND, Packetpipe.Waiting, & $t_{112}^{2}$ \\
\hline 16 & Packetizer.idle, Packetizer.packet, PacketPipe.waiting & $t_{213}^{2}$ \\
\hline 17 & Packetizer.idle, PackePipe.RECV, PacketPipe.waiting & $t_{21}^{2}$ \\
\hline \multirow[t]{2}{*}{18} & Packetizer.idle, PacketPipe.waiting & $t_{26}^{2}$ \\
\hline & Packetizer.idle, PacketPipe.waiting & \\
\hline
\end{tabular}


1. Each basic component and connector are defined as a system module (SysID) with the declaration of variables and necessary rules and operators. Each composition is specified as a system module that including its sub-components and connector that are predefined as a module. All guard conditions in a transition are a (un)conditional rule.

2. Each place is mapped to an operator in the predicate system module (SysID-PREDS). The connection between operators and predicate is established by an equation.

3. Model checking module (SysID-CHECK) is mainly for the initial marking and property specification.

In the translation, system signature such as sorts and operators are declared in the functional module. This translates the places/ports, sorts into algebra in Maude that will be used in the system modules. The dynamic semantics of Petri net can be mapped to the rewriting rules used in Maude. Computationally, the meaning of rewriting rules is to specify local concurrent transitions that can take place in a system if the pattern in the rule's lefthand side matches a fragment of the system state and the rule's condition is satisfied. In that case, the transition specified by the rule can take place, and the matched fragment of the state is transformed into the corresponding instance of the righthand side. Thus an amazing match between semantics of Petri net and rewriting logic can be observed. These are theoretic aspect of the above translation algorithm.

\section{Results}

The basic requirements for the image processing in the distributed web applications are correctness, robustness and reliability. We use model checker of Maude [47] to validate the SO-SAM model obtained from UML architecture description against system properties. After studying models and the errors discovered during the model validation, two main property categories have been selected:

1. Structural properties: this kind of properties is closely related to the topology of 
the model. These properties can be directly verified on the SO-SAM model without animating the transactions. These properties are necessary conditions that ensure the feasibility of the state transitions. If one of them is not fulfilled, we can assert firmly that the communication between ports in UML description cannot happen.

2. Behavioral properties: the dynamic feature of these properties means that they are related to state changing of the system. The evaluation of the dynamic properties are based on the behavior description - Petri nets. Its verification is achieved on a set of places describing a possible evolution of the system. All four properties in section 8.7.2 fall in this group.

The results output from Maude are true for all the above formulae. Most the above formulae are safety properties.

\subsection{Summary}

This section introduced four case studies on the integrated framework. From the experiment results for all case studies, we can tell that the runtime verification do provide a complementary for the model checking to avoid the state explosion problem. Furthermore, we demonstrated extension of the SAM model on the web services with a case study. Finally, we show the model checking application on the UML architecture description. 


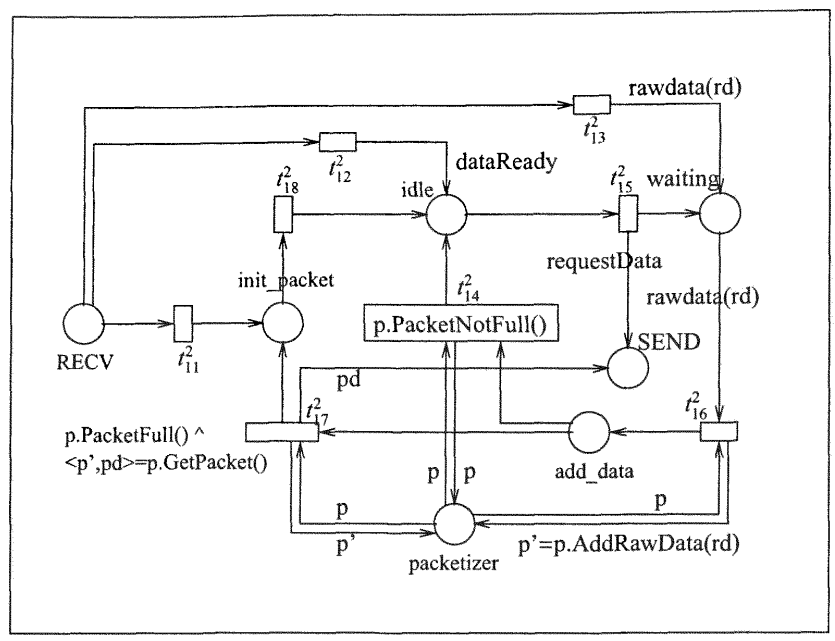

(a) Petri net for Packetizer

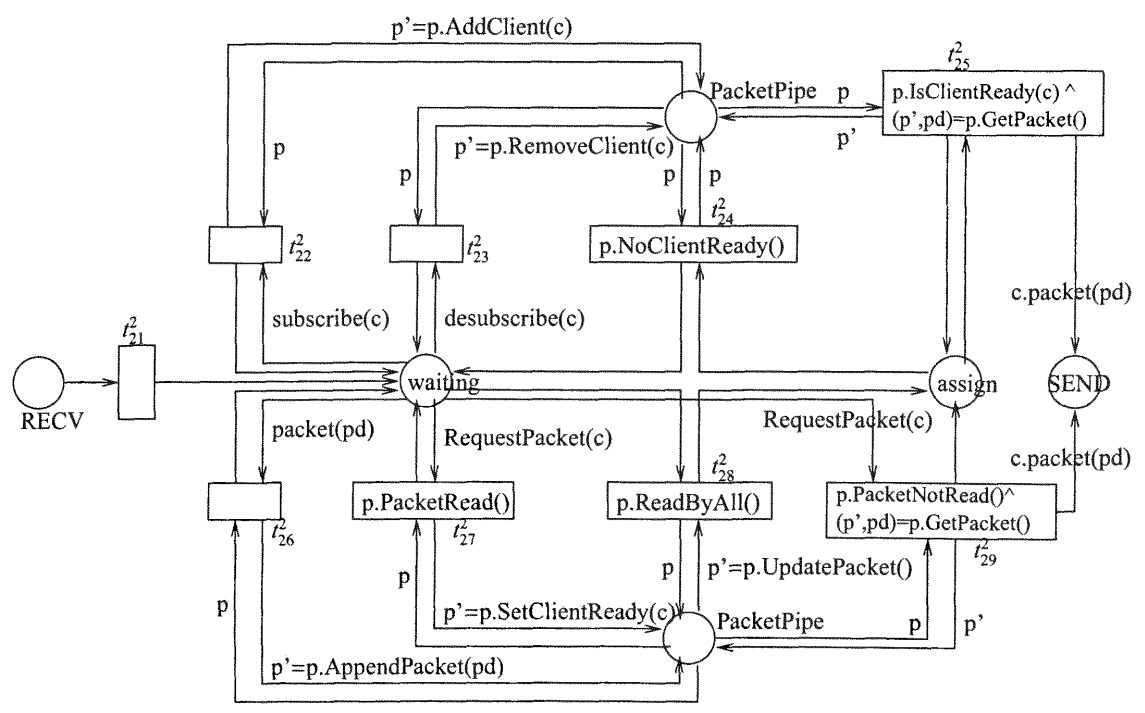

(b) Petri net for PacketPipe

Figure 31: Behavior of Elements (PrT Nets) 


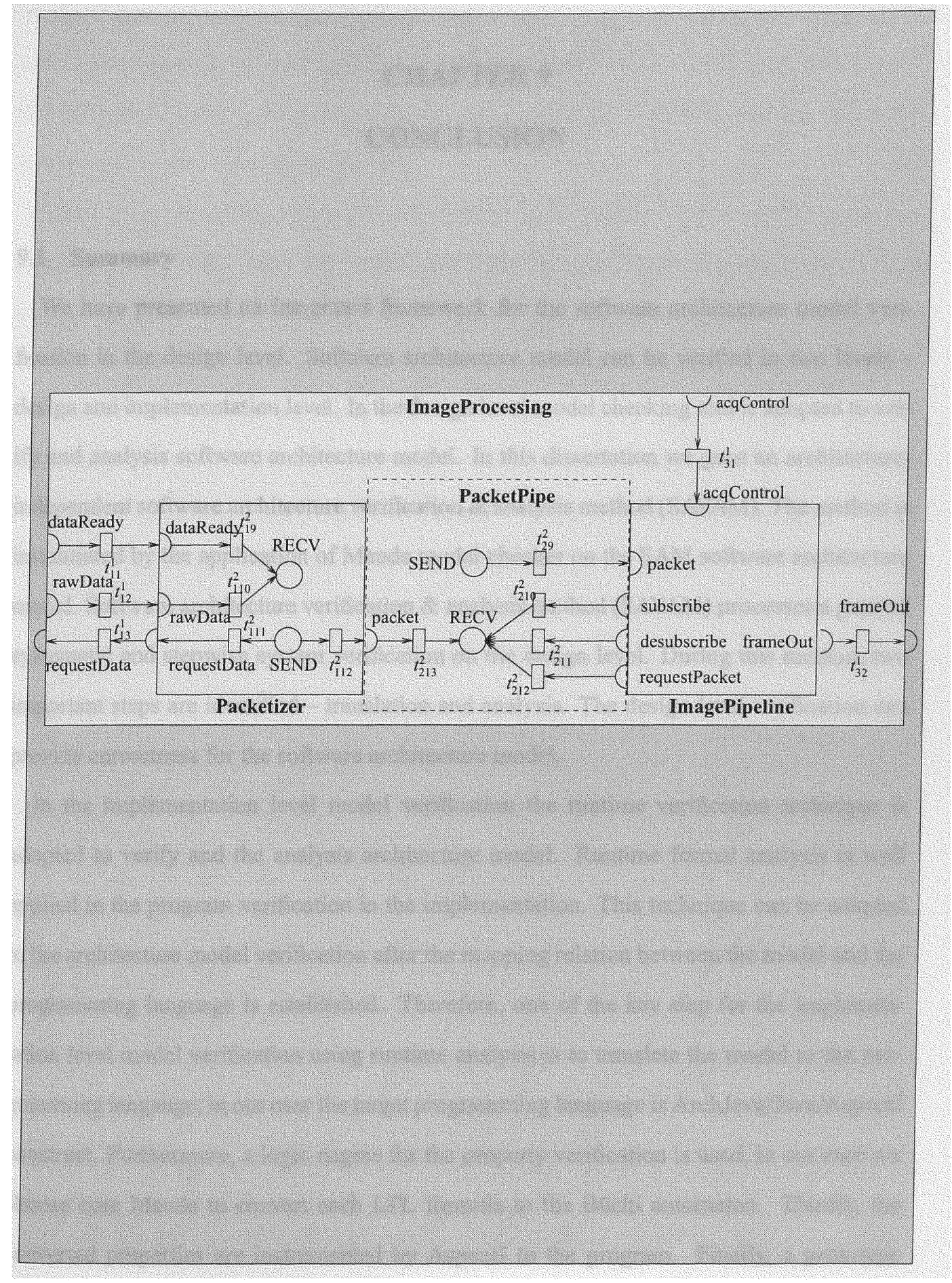

Figure 32: ImageProcessing in SO-SAM 


\section{CHAPTER 9}

\section{CONCLUSION}

\subsection{Summary}

We have presented an integrated framework for the software architecture model verification in the design level. Software architecture model can be verified in two levels design and implementation level. In the design level model checking tool is adopted to verify and analysis software architecture model. In this dissertation we gave an architectureindependent software architecture verification \& analysis method (SAVAM). The method is instantiated by the application of Maude model checker on the SAM software architecture model. Software architecture verification \& analysis method (SAVAM) processes a general systematic and stepwise system verification on the design level. During this method, two important steps are identified - translation and analysis. The design level verification can provide correctness for the software architecture model.

In the implementation level model verification the runtime verification technique is adopted to verify and the analysis architecture model. Runtime formal analysis is well applied in the program verification in the implementation. This technique can be adapted to the architecture model verification after the mapping relation between the model and the programming language is established. Therefore, one of the key step for the implementation level model verification using runtime analysis is to translate the model to the programming langauge, in our case the target programming language is ArchJava/Java/AspectJ construct. Furthermore, a logic engine for the property verification is used, in our case we choose core Maude to convert each LTL formula to the Büchi automaton. Thirdly, the converted properties are instrumented by AspectJ to the program. Finally, a prototype tool - SAM Parser - is developed that uses ArchJava/Java/AspectJ construct and the LTL formula as input and executes runtime checker and the monitored program in parallel to 
verify system properties in the implementation level. Compared with design level verification, implementation level verification ensure the translated code of the model correct. The common feature is verifications in both level check the system against the properties specified in temporal logical formulas.

Two translations from software architecture model are validated based on the interleaving semantics of Petri nets. The validations show the correctness of the translation based on the defined criteria, translation correctness. Without the correctness of the translations it will not make sense for the verification in the implementation level. Even though we can say the code holds the specified properties, but we cannot sure the code is same as the model. Two approaches are presented for the these translations. The first approach is to show the correctness of the translation from SAM model to the Maude program. This approach established a stepwise comparison between the state sequence of SAM behavior model (a PrT net) and the rewriting steps of a rewrite theory constructed from the behavior model (a PrT net). Then a consistency relation can be proved based on the inference rules of the rewrite theory. The second approach is to show the correctness of the translation from SAM model to the java program. First, the java construct for a component/connector in a SAM model has to be restricted to fit for the automatic code generation from the prototype tool - SAM Parser. Then this approach uses a sequential object oriented program language (COORE) syntax to represent the java program. Based on the given operational semantics of the COORE programming language, we define the communication events and constructively define the communication trace on the syntax of the COORE programming language. Moreover, a mapping function between the PrT nets and the java program is defined. Finally, a consistency proof between the PrT nets and the communication trace is established based on the mapping function and the projection of the firing rule for a transition.

It is worth to note that translation validations are not redundant for the runtime analysis applied in the code. The runtime analysis is used to verify the system when the model checking is not able to do it after the system size is increased. 
Finally, we re-identify the significance of the integration framework to ensure the quality of software systems in the design level. One of the advantages of model checking is the exhaustive state space searching for the model. The notorious issue for the model checking is state explosion. Compared with the model checking, runtime analysis is checking the current execution path of a running program against certain properties. The advantage of runtime analysis is that it scales well, and does not suffer the state explosion problem. One issue is it only overlooks the current execution path. Another issue is runtime verification is not good at checking the safety properties due to the infinite execution trace. In this dissertation we demonstrated the integration through the complementary between runtime analysis and model checking on the verification of the software architecture model in the design. This is further confirmed in several case studies.

\subsection{Future Work}

The integrated framework performs model checking and runtime analysis on the software architecture model in the design. We have explored the state explosion problem and the verification of liveness properties during case studies, however, the categorization of different properties and domain systems need to be further analyzed in a future work.

The engagement between model checking and runtime analysis technique highly depends on the system domain and properties specified. Deep analysis and classification of properties and their patterns can identify the engaging point between model checking and runtime analysis. This also can be an extension to the research of the dissertation "An Integrated Framework for Ensuring the Quality of Software Design.” 


\section{LIST OF REFERENCES}

[1] Business Process Execution Language for Web Services (BPEL4WS). Available from http://www.ibm.com/developerworks/library/ws-bpel.

[2] DAML-S and OWL-S. Available from http://www.daml.org/services/owl-s/.

[3] Formal methods publications. Available from http://vl.fmnet.info/pubs/.

[4] Java platform debugger architecture (jpda). Available from http://java.sun.com/products/jpda/index.jsp.

[5] Java tutor. Available from http://java.sun.com.

[6] Payment online. Available from http://www.paymentonline.com/ shopping-cartsoftware.

[7] Simple Object Access Protocol (SOAP), W3C Note 08. Available from http://www.w3.org/TR/SOAP/.

[8] Time rover. Available from http://www.time-rover.com/.

[9] Web Service Choreography Interface (WSCI) 1.0. Available from http://www.w3.org/TR/2002/NOTE-wsci-20020808/.

[10] Web Services Description Language (WSDL) 1.1. Available from http://www.w3.org/TR/wsdl.

[11] Specification and description language. Available from http://www.sdlforum.org/sdl88tutorial/index.html, 1988.

[12] Specification and description language. Available from http://www.iec.org/online/tutorials/sdl/, 1992.

[13] Ariane 5 flight 501 failure. Available from http://www.cs.unibo.it/ laneve/papers/ariane5rep.html, 1996, accessed January 2004.

[14] Discussion of the Object Management Architecture. OMG Document 00-06-41, 1997.

[15] Deduction is forever. Invited Talk at Formal Methods, 1999.

[16] Information technology research: Investing in our future. Available from http://www.itrd.gov/pitac/report/, February 1999.

[17] Archjava Language Reference Manual. Available from http://archjava.fluid.cs.cmu.edu/papers/index.html, 2000, accessed January 2004. 
[18] P. Abercrombie and M. Karaorman. jContractor: Bytecode Instrumentation Techniques for Implementating Design by Contract in Java. In Proceedings of RV'02: Second International Workshop on Runtime Verification, Paris, France, Electronic Notes in Theoretical Computer Science. Elsevier Science, July 2002.

[19] J. Aldrich, C. Chambers, and D. Notkin. Archjava: Connecting Software Architecture to Implementation. In Proceedings of International Conference on Software Engineering, Orlando, FL, USA, May 2002.

[20] J. Aldrich, V. Sazawal, D. Notkin, and C. Chambers. Language support for connector abstractions. In Proceedings of European Conference on Object-Oriented Programming (ECOOP 2003), Darmstadt, Germany, July 21-25 2003.

[21] R. J. Allen. A formal approach to software architecture. PhD thesis, 1997. ChairDavid Garlan.

[22] B. Alpern and F. B. Schneider. Defining liveness. Technical report, Ithaca, NY, USA, 1984.

[23] R. Alur, C. Courcoubetis, T. A. Henzinger, and P.-H. Ho. Hybrid automata: An algorithmic approach to the specification and verification of hybrid systems. In Hybrid Systems I, Lecture Notes in Computer Science, 1993.

[24] R. Alur, J. Esposito, M. Kim, V. Kumar, and I. Lee. Formal modeling and analysis of hybrid systems: A case study in multirobot coordination. In Proceedings of the World Congress on Formal Methods (FM'99), volume 1708 of Lecture Notes in Computer Science, 1999.

[25] R. Alur and T. A. Henzinger. Reactive modules. In Proceedings of the 11th Annual Symposium on Logic in Computer Science (LICS), IEEE Computer Society Press, pages 207-218, 1996.

[26] AspectJ Project. Available from http://eclipse.org/aspectj/.

[27] D. Bartetzko and C. Fischer. Java - Java with Assertions. In Proceedings of RV'01: First International Workshop on Runtime Verification, Paris, France, Electronic Notes in Theoretical Computer Science. Elsevier Science, 2001.

[28] E. Best. Semantics of sequential and parallel programs. Prentice-Hall, Inc., Upper Saddle River, NJ, USA, 1996.

[29] H. S. Bhatt, V. H. Patel, and A. K. Aggarwal. Web enabled client-server model for development environment of distributed image processing. In Proceedings of the First IEEE/ACM International Workshop on Grid Computing (GRID'00), pages 135-145, London, UK, 2000. Springer-Verlag. 
[30] J. Billington, S. Christensen, et al. The Petri Net Markup Language: Concepts, Technology, and Tools. In Proceedings of the 24th International Conference on Applications and Theory of Petri Nets (ICATPN 2003), volume 2679 of Lecture Notes in Computer Science, pages 483-505. Springer-Verlag, June 2003.

[31] S. Blake-Wilson and A. Menezes. Unknown Key-Share Attacks on the Station-toStation (STS) Protocol. Technical Report CORR 98-42, University of Waterloo, 1998.

[32] A. Bouhoula, J.-P. Jouannaud, and J. Meseguer. Specification and proof in membership equational logic. Technical report, SRI International, 1988.

[33] J. P. Bowen and M. G. Hinchey. Ten commandments of formal methods. IEEE Computer, 4, April 1995.

[34] P. Brémond-Grégoire, I. Lee, and R. Gerber. Acsr: An algebra of communicating shared resources with dense time and priorities. In Proceedings of the 4th International Conference on Concurrency Theory (CONCUR'93), pages 417-431, London, UK, 1993. Springer-Verlag.

[35] R. E. Bryant. Graph-based algorithms for Boolean function manipulation. IEEE Transactions on Computers, C-35(8):677-691, Aug. 1986.

[36] R. E. Bryant. Symbolic boolean manipulation with ordered binary decision diagrams. ACM Computing Surveys, 24(3), Sept. 1992.

[37] I. Cerna and R. Pelanek. Relating hierarchy of linear temporal properties to model checking, 2003.

[38] E. Y. Chang, Z. Manna, and A. Pnueli. Characterization of temporal property classes. In Proceedings of the 19th International Colloquium on Automata, Languages and Programming (ICALP'92), pages 474-486, London, UK, 1992. Springer-Verlag.

[39] F. Chen and G. Rosu. Towards monitoring-oriented programming: A paradigm combining specification and implementation. Electronic Notes in Theoretical Computer Science, 89(2), 2003.

[40] S.-W. Cheng and D. Garlan. Mapping Architectural Concepts to UML-RT. In Proceedings of International Conference on Parallel and Distributed Processing Techniques and Applications (PDPTA'2001), June 2001.

[41] S. E. Chodrow and M. G. Gouda. Implementation of the sentry system. SoftwarePractice and Experience, 25(4):373-387, 1995. 
[42] S. E. Chodrow and M. G. Gouda. Sentries for the execution of concurrent programs. In ICDCS, pages 11-18, 1996.

[43] D. Clarke and I. Lee. Automatic test generation for the analysis of a real-time system: Case study. In Proceedings of 3rd IEEE Real-time Technology and Applications Symposium, June 1997.

[44] E. M. Clarke, E. A. Emerson, and A. P. Sistla. Automatic verification of finitestate concurrent systems using temporal logic specifications. ACM Transactions on Programming Languages Systems, 8(2):244-263, 1986.

[45] E. M. Clarke, O. Grumberg, and D. A. Peled. Computer Aided Verification. The MIT Press, January 2000.

[46] E. M. Clarke and J. M. Wing. Formal methods: State of the art and future directions. ACM Computing Surveys, 4, 1996.

[47] M. Clavel, F. Durán, S. Eker, P. Lincoln, N. Martí-Oliet, J. Meseguer, and J. F. Quesada. Maude: specification and programming in rewriting logic. Theoretical Computer Science, 285(2):187-243, 2002.

[48] M. Clavel, F. Durán, S. Eker, P. Lincoln, N. Martí-Oliet, J. Meseguer, and C. Talcott. Maude manual, 2004.

[49] A. Corradini and F. Gadducci. Rational term rewriting. Lecture Notes in Computer Science, 1378:156-171, 1998.

[50] M. Dahm. Byte code engineering with the BCEL API. Technical report, institution of information, April 2001.

[51] W. Diffie, P. C. van Oorschot, and M. J. Wiener. Authentication and Authenticated Key Exchanges. Designs, Codes and Cryptography, 2, 1992.

[52] L. K. Dillon, G. Kutty, L. E. Moser, P. M. Melliar-Smith, and Y. S. Ramakrishna. A graphical interval logic for specifying concurrent systems. ACM Transactions on Software Engineering and Methodology, 3(2):131-165, 1994.

[53] L. K. Dillon and Q. Yu. Oracles for checking temporal properties of concurrent systems. In SIGSOFT '94: Proceedings of the 2nd ACM SIGSOFT symposium on Foundations of software engineering, pages 140-153, New York, NY, USA, 1994. ACM Press.

[54] L. Dobrica and E. Niemelä. A survey on software architecture analysis methods. IEEE Transaction on Software Engineering, 28(7):638-653, 2002. 
[55] Z. Dong, Y. Fu, and X. He. Deriving Hierarchical Predicate/Transition Nets from Statechart Diagrams. In Proceedings of The 15th International Conference on Software Engineering and Knowledge Engineering (SEKE2005), 2003.

[56] Z. Dong, Y. Fu, and X. He. An Integrated Runtime Monitoring Framework for Software Architecture Design. In Proceedings of the Software Engineering and Applications (SEA 2005), 2005.

[57] Z. Dong, Y. Fu, and X. He. Automated runtime validation of software architecture design. In Proceedings of Second International Conference of Distributed Computing and Internet Technology (ICDCIT 2005), volume 3816 of Lecture Notes in Computer Science, pages 446-457. Springer, 2005.

[58] B. Dutertre and V. Stavridou. Formal requirements analysis of an avionics control system. Software Engineering, 23(5):267-278, 1997.

[59] M. B. Dwyer, G. S. Avrunin, and J. C. Corbett. Property specification patterns for finite-state verification. In Proceedings of 2nd Workshop on Formal Methods in Software Practice (FMSP-98), New York. ACM Press.

[60] O. G. E.Clarke and D.Long. Verification tools for finite-state concurrent systems. In Proceedings of In A Decade of Concurrency - Reflections and Perspectives, volume 803 of Lecture Notes in Computer Science, 1994.

[61] A. Egyed. Automating Architectural View Integration in UML. Technical Report USCCSE-99511, Center for Software Engineering, University of Southern California,Los Angeles, CA, 1999.

[62] A. Egyed and N. Medvidovic. Extending Architectural Representation in UML with View Integration. In Proceedings of the 2nd International Conference on the Unified Modeling Language, pages 2-16, October 1999.

[63] H. Ehrig and B. Mahr. Fundamentals of Algebraic Specification 1: Equations and Initial Semantics. Springer-Verlag, 1985.

[64] H. Ehrig and B. Mahr. Fundamentals of Algebraic Specification 2: Module Specifications and Constraints. Springer-Verlag, 1990.

[65] H. Ehrig, J. Padberg, and L. Ribeiro. Algebraic High-Level Nets: Petri Nets Revisited. In Proceedings of Recent Trends in Data Type Specification, 9th Workshop on Specification of Abstract Data Types Joint with the 4th COMPASS Workshop, volume 785 of Lecture Notes in Computer Science, pages 188-206. Springer, 1994. 
[66] S. Eker, J. Meseguer, and A. Sridharanarayanan. The maude LTL model checker. In Proceedings of the 4th International Workshop on Rewriting Logic and Its Applications (WRLA'02), volume 71 of Electronic Notes in Theoretical Computer Science, Amsterdam, September 2002. Elsevier.

[67] J. Esparza and S. Romer. An unfolding algorithm for synchronous products of transition systems. In Proceedings of International Conference on Concurrency Theory, volume 1664 of Lecture Notes in Computer Science, pages 2-20, 1999.

[68] J. Esparza, S. Romer, and W. Vogler. An improvement of McMillan's unfolding algorithm. In Proceedings of Tools and Algorithms for Construction and Analysis of Systems, pages 87-106, 1996.

[69] B. Finkbeiner and H. Sipma. Checking finite traces using alternating automata. Formal Methods in System Design, 24(2):101-127, 2004.

[70] C. C. Frederiksen. Correctness of classical compiler optimizations using CTL. In Proceedings of theETAPS 2002 : European Joint Conference on Theory and Practice of Software, pages 41-55, 2002.

[71] Y. Fu, Z. Dong, and X. He. A Methodology of Automated Realization of a Software Architecture Design. In Proceedings of The Seventeenth International Conference on Software Engineering and Knowledge Engineering (SEKE2005), 2005.

[72] Y. Fu, Z. Dong, and X. He. An Approach to Validation of Software Architecture Model. In Proceedings of the 12th Asia-Pacific Software Engineering Conference (APSEC'05), 2005.

[73] Y. Fu, Z. Dong, and X. He. An Integrated Runtime Monitoring Framework for Software Architecture Design. In Proceedings of the Software Engineering and Applications (SEA'05), 2005.

[74] Y. Fu, Z. Dong, and X. He. An Approach to Web Services Oriented Modeling and Validation. In Proceedings of the 28th ICSE workshop on Service Oriented Software Engineering (SOSE2006), 2006.

[75] Y. Fu, Z. Dong, and X. He. Formalizing and Validating UML Architecture Description of Web Systems. In Proceedings of of ICWE06 workshop: Model-Driven Web Engineering (MDWE'06), Palo Alto, CA, USA, 2006.

[76] Y. Fu, Z. Dong, and X. He. A method for realizing software architecture design. In Proceedings of the Sixth International Conference on Quality Software(QSIC'06), pages 57-64, Washington, DC, USA, 2006. IEEE Computer Society. 
[77] Y. Fu, Z. Dong, and X. He. Modeling, Validating and Automating Composition of Web Services. In Proceedings of The Sixth International Conference on Web Engineering (ICWE 2006), 2006.

[78] G. C. Gannod and S. Gupta. An automated tool for analyzing petri nets using spin. In ASE '01: Proceedings of the 16th IEEE international conference on Automated software engineering, page 404, Washington, DC, USA, 2001. IEEE Computer Society.

[79] D. Garlan, S.-W. Cheng, and A. J. Kompanek. Reconciling the Needs of Architectural Description with Object-Modeling Notations. Science of Computer Programming, 44(1):23-49, July 2002.

[80] M.-C. Gaudel. Testing can be formal, too. In TAPSOFT '95: Proceedings of the 6th International Joint Conference CAAP/FASE on Theory and Practice of Software Development, pages 82-96, London, UK, 1995. Springer-Verlag.

[81] I. Georgiadis. Design Issues in the Mapping of the Darwin ADL to Java using RMI as the Communication Substrate. Available from http://www.doc.ic.ac.uk/ igeozg/Project/Darwin, Nov 1999.

[82] D. Giannakopoulou and K. Havelund. Automata-based verification of temporal properties on running programs. In ASE '01: Proceedings of the 16th IEEE International Conference on Automated Software Engineering, page 412, Washington, DC, USA, 2001. IEEE Computer Society.

[83] S. Glesner, R. Geï, and B. Boesler. Verified code generation for embedded systems. In Proceedings of the COCV-Workshop (Compiler Optimization meets Compiler Verification), volume 65. Electronic Notes in Theoretical Computer Science (ENTCS), 5th European Conferences on Theory and Practice of Software (ETAPS 2002),, April 132002 .

[84] J. A. Goguen and J. Meseguer. Order-sorted algebra I: Equational deduction for multiple inheritance, overloading, exceptions and partial operations. Theoretical Computer Science, 105(2):217-273, 1992.

[85] H. Gomaa. Designing Concurrent, Distributed, and Real-Time Applications with UML. Addison-Wesley Professional, 2000.

[86] G. Goos and W. Zimmermann. Verification of compilers. In Proceedings of Correct System Design, pages 201-230, 1999.

[87] M. M. Gorlick and R. R. Razouk. Using Weaves for Software Construction and Analysis. In Proceedings of the 13th International Conference on Software Engineering (ICSEI3), Austin, TX, USA, May 1991. 
[88] K. H. Grigore Rosu. Synthesizing dynamic programming algorithms from linear temporal logic formulae. Research Report TR 01-15, Siebel Center, Department of Computer Science, University of Illinois at Urbana-Champaign, May 2001.

[89] K. Havelund. Using runtime analysis to guide model checking of java programs. In Proceedings of the 7th International SPIN Workshop on SPIN Model Checking and Software Verification, pages 245-264, London, UK, 2000. Springer-Verlag.

[90] K. Havelund, S. Johnson, and G. Rosu. Specification and error pattern based program monitoring. In Proceedings of European Space Agency Workshop on On-Board Autonomy, Noordwijk, Holland, October 2001.

[91] K. Havelund and T. Pressburger. Model checking java programs using java pathfinder. International Journal on Software Tools for Technology Transfer, 2(4), April 2000.

[92] K. Havelund and G. Rosu. Monitoring Java Programs with Java PathExplorer. In Proceedings of the 18th International Conference on Software Enginnering, Paris, France, Electronic Notes in Theoretical Computer Science, pages 97-114, July 2001.

[93] K. Havelund and G. Rosu. Monitoring programs using rewriting. In Proceedings of International Conference on Automated Software Engineering (ASE'01), pages 135-143. Institute of Electrical and Electronics Engineers, 2001. Coronado Island, California.

[94] K. Havelund and G. Rosu. Synthesizing monitors for safety properties. In Proceedings of Tools and Algorithms for Construction and Analysis of Systems, pages 342-356, 2002.

[95] K. Havelund and G. Rosu. An overview of the runtime verification tool java pathexplorer. Journal of Formal Methods in System Design, 2004.

[96] X. He. A Formal Definition of Hierarchical Predicate Transition Nets. volume 1091 of Lecture Notes in Computer Science, pages 212 - 229, 1996.

[97] X. He and Y. Deng. A Framework for Specifying and Verifying Software Architecture Specifications in SAM. volume 45 of The Computer Journal, pages 111-128, 2002.

[98] X. He, J. Ding, and Y. Deng. Model checking software architecture specifications in sam. In Proceedings of the 14th international conference on Software engineering and knowledge engineering (SEKE'02), volume 27 of ACM International Conference Proceeding Series, pages 271-274, New York, NY, USA, 2002. ACM Press. 
[99] X. He, H. Yu, T. Shi, J. Ding, and Y. Deng. Formally analyzing software architectural specifications using sam. Journal of Systems and Software, 71(1-2):11-29, 2004.

[100] R. Heckel, M. Lohmann, and S. Thöne. Towards a UML Profile for Service-Oriented Architectures. Available from http://citeseer.csail.mit.edu/heckel03towards.html.

[101] R. Heckel, H. Voigt, J. Küster, and S. Thöne. Towards Consistency of Web Service Architectures. Available from http://www.upb.de/cs/agengels/Papers/2003/HeckelVoigtKuesterThoene-SCI03.pdf.

[102] T. Hoare. The verifying compiler: A grand challenge for computing research. Journal of the ACM, 50(1):63-69, 2003.

[103] C. Hofmeister, R. Nord, and D. Soni. Applied Software Architecture. Addison Wesley, 2000.

[104] C. Hofmeister, R. L. Nord, and D. Soni. Describing Software Architecture with UML. In Proceedings of the TC2 1st Working IFIP Conference on Software Architecture (WICSA1), pages $145-160,1999$.

[105] G. Holzmann and D. Peled. An improvement in formal verification. In Proceedings of Formal Description Techniques, FORTE94, pages 197-211, Berne, Switzerland, October 1994. Chapman \& Hall.

[106] G. J. Holzmann. The Model Checker SPIN. IEEE Transactions on Software Engineering, 23(5), May 1997.

[107] R. Hull, M. Benedikt, V. Christophides, and J. Su. Eservices: A look behind the curtain. In Proceedings of the International Symposium on Principles of Database Systems (PODS). ACM Press, June 2003.

[108] IBM Coorperation. Web Services Flow Language (WSFL) 1.1. Available from www-3.ibm.com/software/ solutions/webservices/pdf/WSFL.pdf.

[109] F. Jahanian and A. Goyal. A formalism for monitoring real-time constraints at runtime. In Proceedings of 20th International Symposium on Fault-Tolerant Computing (FTCS-20), pages 148-155, 1990.

[110] C. Jeffery, W. Zhou, K. Templer, and M. Brazell. A lightweight architecture for program execution monitoring. SIGPLAN Notices, 33(7):67-74, 1998.

[111] C. L. Jeffery. A Framework for Monitoring Program Execution. PhD thesis, CS Department University of Arizona, 1993. 
[112] E. B. Johnsen and O. Owe. An asynchronous communication model for distributed concurrent objects. In Proceedings of the Software Engineering and Formal Methods, Second International Conference on (SEFM'04), pages 188-197, Washington, DC, USA, 2004. IEEE Computer Society.

[113] J.R. Burch, E.M. Clarke, K.L. McMillan, D.L. Dill, and L.J. Hwang. Symbolic Model Checking: $10^{20}$ States and Beyond. In Proceedings of the Fifth Annual IEEE Symposium on Logic in Computer Science, pages 1-33, Washington, D.C., 1990. IEEE Computer Society Press.

[114] M. Karaorman and J. Freeman. jMonitor: Java Runtime Event Specification and Monitoring Library. In Proceedings of RV'04: Fourth International Workshop on Runtime Verification, Paris, France, Electronic Notes in Theoretical Computer Science. Elsevier Science, April 2004.

[115] M. Kaufmann and J. S. Moore. The boyer-moore theorem prover and its interactive enhancement. Computers and Mathematics with Applications, 29(2):27-62, 1995.

[116] R. Kazman, G. Abowd, L. Bass, and P. Clements. Scenario-based analysis of software architecture. IEEE Software, 13(6):47-55, November 1996.

[117] R. Kazman, L. J. Bass, M. Webb, and G. D. Abowd. SAAM: A method for analyzing the properties of software architectures. In Proceedings of International Conference on Software Engineering, pages 81-90, 1994.

[118] G. Kiczales, J. Lamping, A. Mendhekar, C. Maeda, C. V. Lopes, J.-M. Loingtier, and J. Irwin. Aspect-Oriented Programming. In Proceedings of European Conference on Object-Oriented Programming (ECOOP). Springer-Verlag, 1997.

[119] M. Kim, S. Kannan, I. Lee, and O. Sokolsky. Java-MaC: a Run-time Assurance Tool for Java. In Proceedings of of RV'01: First International Workshop on Runtime Verification, Paris, France, Electronic Notes in Theoretical Computer Science. Elsevier Science, 2001.

[120] K. Korenblat, O. Grumberg, and S. Katz. Translations between textual transition systems and petri nets. In Proceedings of the Third International Conference on Integrated Formal Methods (IFM'02), pages 339-359, London, UK, 2002. SpringerVerlag.

[121] R. P. Kurshan. Reducibility in analysis of coordination. volume 103 of Lecture Notes in Computer Science, pages 19-39, 1987.

[122] R. P. Kurshan. Computer-aided verification of coordinating processes: the automata-theoretic approach. Princeton University Press, Princeton, NJ, USA, 1994. 
[123] L. Lamport. Proving the correctness of multiprocess programs. IEEE Transactions on Software Engineering, 3(2):125-143, 1977.

[124] L. Lamport. An axiomatic semantics of concurrent programming languages. pages 77-122, 1985.

[125] H. B. Lee and B. G. Zorn. BIT: A tool for instrumenting java bytecodes. In Proceedings of the USENLX Symposium on Internet Technologies and Systems,Monterey, California, December 1997.

[126] I. Leey, S. Kannan, M. Kim, O. Sokolsky, and M. Viswanathan. Runtime assurance based on formal specifications. In Proceedings of International Conference on Parallel and Distributed Processing Techniques and Applications, 1999.

[127] N. G. Leveson. Safeware: system safety and computers. ACM Press, New York, NY, USA, 1995.

[128] S. W. Lewandowski and X. He. Generating Code for Hierarchical Predicate Transition Net Based Designs. In Proceedings of the 12th International Conference on Software Engineering $\mathcal{E}$ Knowledge Engineering, pages 15-22, Chicago, U.S.A., July 2000.

[129] G. Liu and A. K. Mok. Implementation of JEM - A java composite event package. In Proceedings of the Fifth IEEE Real-Time Technology and Applications Symposium (RTAS'99), page 68, Washington, DC, USA, 1999. IEEE Computer Society.

[130] C. M. Lott. Correctness is congruent with quality. Software Engineering Notes, 15(5):19-20, October 1990.

[131] D. C. Luckham and J. Vera. An Event Based Architecture Definition Language. IEEE Transactions on Software Engineering, 21(9), 1995.

[132] P. L. M. Clavel, S. Eker and J. Meseguer. Principles of Maude. volume 4 of Electronic Notes in Theoretical Computer Science. Elsevier Science Publishers, 1996.

[133] J. Magee, N. Dulay, S. Eisenbach, and J. Kramer. Specifying Distributed Software Architectures. In Proc. 5th European Software Engineering Conf. (ESEC 95), volume 989, Sitges, Spain. Springer-Verlag, Berlin.

[134] D. Mahrenholz, O. Spinczyk, and W. Schröder-Preikschat. Program instrumentation for debugging and monitoring with aspectc++. In Fifth IEEE International Symposium on Object-Oriented Real-Time Distributed Computing, April 2002. 
[135] Z. Manna and A. Pnueli. A hierarchy of temporal properties (invited paper, 1989). In Proceedings of the ninth annual ACM symposium on Principles of distributed computing (PODC'90), pages 377-410, New York, NY, USA, 1990. ACM Press.

[136] Z. Manna and A. Pnueli. The Temporal Logic of Reactive and Concurrent Systems. Springer-Verlag New York, Inc., New York, NY, USA, 1992.

[137] Z. Manna and A. Pnueli. Temporal Logic of Reactive and Concurrent Systems. Springer, 1992.

[138] K. L. McMillan. Symbolic Model Checking An Approach to the State Explosion Problem. PhD thesis, Carnegie Mellon University, May 1992.

[139] K. L. McMillan. Symbolic Model Checking. Springer, July 1993.

[140] N. Medvidovic, A. Egyed, and D. S. Rosenblum. Round-Trip Software Engineering Using UML:From Architecture to Design and Back. In Proceedings of the 2nd Workshop on Object-Oriented Reengineering, pages 1-8, September 1999.

[141] N. Medvidovic and D. S. Rosenblum. Assessing the Suitability of a Standard Design Method for Modeling Software Architectures. In Proceedings of the 1st IFIP Working Conference on Software Architecture, Feburary 1999.

[142] N. Medvidovic and R. N. Taylor. A classification and comparison framework for software architecture description languages. Software Engineering, 26(1):70-93, 2000.

[143] T. H. G. Megson. Structural and Stress Analysis. Butterworth-Heinemann, 2005.

[144] J. Meseguer. Conditional rewriting logic as a unified model of concurrency. volume 96, pages 73-155, 1992.

[145] J. Meseguer. Rewriting logic as a semantic framework for concurrency: a progress report. In Proceedings of International Conference on Concurrency Theory, pages 331-372, 1996.

[146] J. Meseguer. Membership algebra as a logical framework for equational specification. In Proceedings of Workshop on Algebraic Development Techniques (WADT97), Lecture Notes in Computer Science, pages 18-61, June 1998.

[147] J. Meseguer and G. Rosu. Rewriting Logic Semantics: From Language Specifications to Formal Analysis Tools. In Proceedings of Automated Reasoning, Lecture Notes in Computer Science, pages 1-44. Springer, June 2004. 
[148] Microsoft Coorperation. XLANG - Web Services for Business Process Design. Available from http://www.gotdotnet.com/team/ xml_wsspecs/xlang-c/default.htm.

[149] R. Milner. Communication and concurrency. Prentice-Hall, Inc., Upper Saddle River, NJ, USA, 1989.

[150] A. K. Mok and G. Liu. Early detection of timing constraint violation at runtime. In Proceedings of the IEEE Real-Time Systems Symposium, pages 176-, 1997.

[151] A. K. Mok and G. Liu. Efficient run-time monitoring of timing constraints. In 3rd IEEE Real-Time Technology and Applications Symposium (RTAS '97), pages 252$262,1997$.

[152] M. Moriconi, X. Qian, and R. A. Riemenschneider. Correct Architecture Refinement. IEEE Transactions on Software Engineering, 21(5), 1995.

[153] T. Murata. Petri Nets: Properties, Analysis and Applications. In Proceedings of the IEEE, 77(4):541-580, 1989.

[154] G. J. Myers. Software Reliability: Principles and Practices. Wiley, September 1976.

[155] G. Naumovich and L. A. Clarke. Classifying properties: an alternative to the safetyliveness classification. In Proceedings of the 8th ACM SIGSOFT international symposium on Foundations of software engineering (SIGSOFT'00/FSE-8), pages 159168, New York, NY, USA, 2000. ACM Press.

[156] G. C. Necula. Proof-carrying code. In Proceedings of Conference Record of POPL'97: The 24th ACM SIGPLAN-SIGACT Symposium on Principles of Programming Languages, pages 106-119, Paris, France, Jan 1997.

[157] G. C. Necula. Translation validation for an optimizing compiler. ACM SIGPLAN Notices, 35(5):83-94, 2000.

[158] G. C. Necula and P. Lee. The design and implementation of a certifying compiler. In Proceedings of the 1998 ACM SIGPLAN Conference on Prgramming Language Design and Implementation (PLDI), pages 333-344, 1998.

[159] N. Nethercote and A. Mycroft. Redux: A dynamic dataflow tracer. In Electronic Notes in Theoretical Computer Science. Elsevier, July 2003.

[160] R. A. Olsson, R. H. Cawford, and W. W. Ho. A dataflow approach to event-based debugging. Software - Practice and Experience, 21(2):209-230, 1991.

[161] R. A. Olsson, R. H. Crawford, and W. W. Ho. Dalek: A GNU, Improved Programmable Debugger. 
[162] J. Padberg and H. Ehrig. Petri Net Modules in the Transformation-based Component Framework. Journal of Logic and Algebraic Programming, 67(1-2):198-225, 2006.

[163] J. Padberg, H. Ehrig, and L. Ribiero. Algebraic High-Level Net Transformation Systems. Mathematical Structures in Computer Science, 5:217-256, 1995.

[164] J. S. Paul Clements, Len Bass. Documenting Software Architectures: Views and Beyond. Addison-Wesley, January 2003.

[165] D. Peled. Combining partial order reductions with on-the-fly model-checking. In Proceedings of the 6th International Conference on Computer Aided Verification (CAV'94), pages 377-390, London, UK, 1994. Springer-Verlag.

[166] W. Penczek, M. Szreter, R. Gerth, and R. Kuiper. Partial order reductions preserving simulations, 1999.

[167] D. E. Perry and A. L. Wolf. Foundations for the study of software architecture. $A C M$ SIGSOFT Software Engineering Notes, 17(4):40-52, 1992.

[168] B. C. Pierce. Basic Category Theory for Computer Scientists (Foundations of Computing). The MIT Press, August 1991.

[169] C. Pierik. Validation Techniques for Object-Oriented Proof Outlines. PhD thesis, Computer Science Department, Utrecht University, May 2006.

[170] C. Pierik and F. S. de Boer. A proof outline logic for object-oriented programming. Theoretical Computer Science, 343(3):413-442, 2005.

[171] A. Pnueli. The temporal logic of programs. In Proceedings of the 18th IEEE Symposium on Foundations of Computer Science, 1977.

[172] A. Pnueli, O. Shtrichman, and M. Siegel. The code validation tool CVT: Automatic verification of a compilation process. International Journal on Software Tools for Technology Transfer, 2(2):192-201, 1998.

[173] A. Pnueli, M. Siegel, and E. Singerman. Translation validation. Lecture Notes in Computer Science, 1384:151-166, 1998.

[174] A. N. Prior. Temporal Logic of Reactive and Concurrent Systems. Greenwood Press Reprint, 1979.

[175] M. Rinard. Credible compilers. Technical Report MIT/LCS/TR-776, 1999.

[176] X. Rival. Abstract interpretation-based certification of assembly code. In Proceedings of the 4th International Conference on Verification, Model Checking, and 
Abstract Interpretation (VMCAI'03), pages 41-55, London, UK, 2003. SpringerVerlag.

[177] G. Rosu and K. Havelund. Rewriting-Based Techniques for Runtime Verification. Journal of Automated Software Engineering, 2004.

[178] J. R. Rumbaugh, M. R. Blaha, W. Lorensen, F. Eddy, and W. Premerlani. ObjectOriented Modeling and Design. Prentice Hall, October 1990.

[179] Seventh Workshop on Runtime Verification (RV'07), Barcelona, Spain. Electronic Notes in Theoretical Computer Science, Volume 113, 2004.

[180] J. Saldhana, S. M. Shatz, and Z. Hu. Formalization of Object Behavior and Interactions From UML Models. International Journal of Software Engineering and Knowledge Engineering, pages 643-673, 2001.

[181] S. Sankar. Automatic runtime consistency checking and debugging of formally specified programs. PhD thesis, 1989. Adviser-David C. Luckham.

[182] T. Savor and R. E. Seviora. An approach to automatic detection of software failures in real-time systems. In Proceedings of IEEE Real Time Technology and Applications Symposium, pages 136-146, 1997.

[183] A. Sen and V. K. Garg. Partial order trace analyzer (pota) for distributed programs. In Proceedings of the Third International Workshop on Runtime Verification (RV'03), volume 89 of Electronic Notes in Theoretical Computer Science. Elsevier Science, 2003.

[184] K. Sen, G. Rosu, and G. Agha. Runtime safety analysis of multithreaded programs. In Proceedings of the 9th European software engineering conference held jointly with 11th ACM SIGSOFT international symposium on Foundations of software engineering (ESEC/FSE-11), pages 337-346, New York, NY, USA, 2003. ACM Press.

[185] B. Shannon, M. Hapner, V. Matena, J. Davidson, J. Davidson, and L. Cable. Java 2 Platform, Enterprise Edition: Platform and Component Specifications. Pearson Education, 2000.

[186] M. Shaw, R. DeLine, D. V. Klein, T. L. Ross, D. M. Young, and G. Zelesnik. Abstractions for Software Architecture and Tools to Support Them. IEEE Transactions on Software Engineering, April 1995.

[187] M. Shaw and D. Garlan. Software Architecture: Perspectives on an Emerging Discipline. Prentice Hall, 1996. 
[188] R. Snodgrass. A relational approach to monitoring complex systems. ACM Transactions on Computer Systems (TOCS), 6(2):157-195, 1988.

[189] R. Sosič. Dynascope: A tool for program directing. In Proceedings of SIGPLAN'92 Conference on Programming Language Design and Implementation, 1992.

[190] R. Sosič. Design and implementation of dynascope, a directing platform for compiled programs. Computing Systems, 8(2), 1995.

[191] R. Sosič. A procedural interface for program directing. Software - Practice and Experience, 25(7), 1995.

[192] M. M. Sriram Sankar. Concurrent runtime monitoring of formally specified programs. IEEE Computer, 26(3):32-41, March 1993.

[193] K. S. Templer and C. Jeffery. A configurable automatic instrumentation tool for ansi c. In Proceedings of the IEEE 13th International Conference on Automated Software Engineering, ASE'98, Honolulu Hawaii, USA, October 1998.

[194] A. Valmari. A stubborn attack on state explosion. In Proceedings of the 2nd International Workshop on Computer Aided Verification (CAV'90), pages 156-165, London, UK, 1991. Springer-Verlag.

[195] W. M. P. van der Aalst, K. M. van Hee, and R. A. van der Toorn. Component-based software architectures: A framework based on inheritance of behavior. Science of Computer Programming, 42(2-3):129-171, 2002.

[196] M. Y. Vardi. Alternating automata:checking truth and validity for temporal logics. In Proceedings of the 14th International Conference on Automated Deduction, Lecture Notes in Computer Science, pages 191-206, 1997.

[197] S. Vestal. MetaH User's Manual, 1998.

[198] J. Wang, X. He, and Y. Deng. Introducing Software Architecture Specification and Analysis in SAM through an Example. Information and Software Technology, 41(7):451-467, 1999.

[199] S. Williams and C. Kindel. The Component Object Model: Technical Overview. Dr. Dobbs Journal, December 1994.

[200] J. M. Wing. A Specifier's Introduction to Formal Methods. IEEE Computer, 23(9), September 1990. 
[201] F. Xie, V. Levin, R. P. Kurshan, and J. C. Browne. Translating Software Designs for Model Checking. In Proceedings of 7 th International Conference Fundamental Approach to Software Engineering (FASE), volume 2984, pages 324-338. SpringerVerlag, 2004.

[202] W. J. Yeh and M. Young. Compositional reachability analysis using process algebra. In Proceedings of the symposium on Testing, analysis, and verification (TAV4), pages 49-59, New York, NY, USA, 1991. ACM Press.

[203] Q. Zhang, J. Wu, and H. Zhu. Tool support to model-based quality analysis of software architecture. compsac, 1:121-128, 2006.

[204] J. Zhao. Robust Object-oriented Systems Analysis. Resources on software development process consulting service.

[205] L. Zuck, A. Pnueli, Y. Fang, B. Goldberg, and Y. Hu. Translation and run-time validation of optimized code. 


\section{APPENDIX A}

\section{MAUDE CODE FOR COFFEE MACHINE}

The following is the functional module for the coffee machine example demonstrated in the Chapter 8:

fmod CM-SIGNATURE is

protecting INT .

sort Marking .

op _- : Marking Marking $\rightarrow$ Marking [assoc comm id: null] .

op null : $\rightarrow$ Marking .

op request-ch : Int Int $\rightarrow$ Marking .

op price : Int Int $\rightarrow$ Marking .

op save : Int Int $\rightarrow$ Marking .

op pay-return-ch : Bool $\rightarrow$ Marking .

op coffee-request-ch : Int $\rightarrow$ Marking .

op coin-back-ch : Int $\rightarrow$ Marking .

op IntToMarking : Int $\rightarrow$ Marking .

*** (brewing facility)

op coffee-request-bf : Int $\rightarrow$ Marking .

op ready-bf : Bool $\rightarrow$ Marking .

op storage : Int Int $\rightarrow$ Marking .

op pay-return-bf : Bool $\rightarrow$ Marking .

op coffee-serve-bf : Int $\rightarrow$ Marking .

***(CMInterface)

op request-cmi : Int Int $\rightarrow$ Marking .

op money-cmi : Int $\rightarrow$ Marking .

op coffeetype-cmi : Int $\rightarrow$ Marking .

op sig : Bool $\rightarrow$ Marking .

op ready-cmi : Bool $\rightarrow$ Marking .

op change-cmi : Int $\rightarrow$ Marking .

op coin-back-cmi : Int $\rightarrow$ Marking .

op coffee-serve-cmi : Int $\rightarrow$ Marking .

op ready-enjoy-cmi : Int $\rightarrow$ Marking .

endfm 
The following is the system module for coffee machine example demonstrated in the Chapter 8:

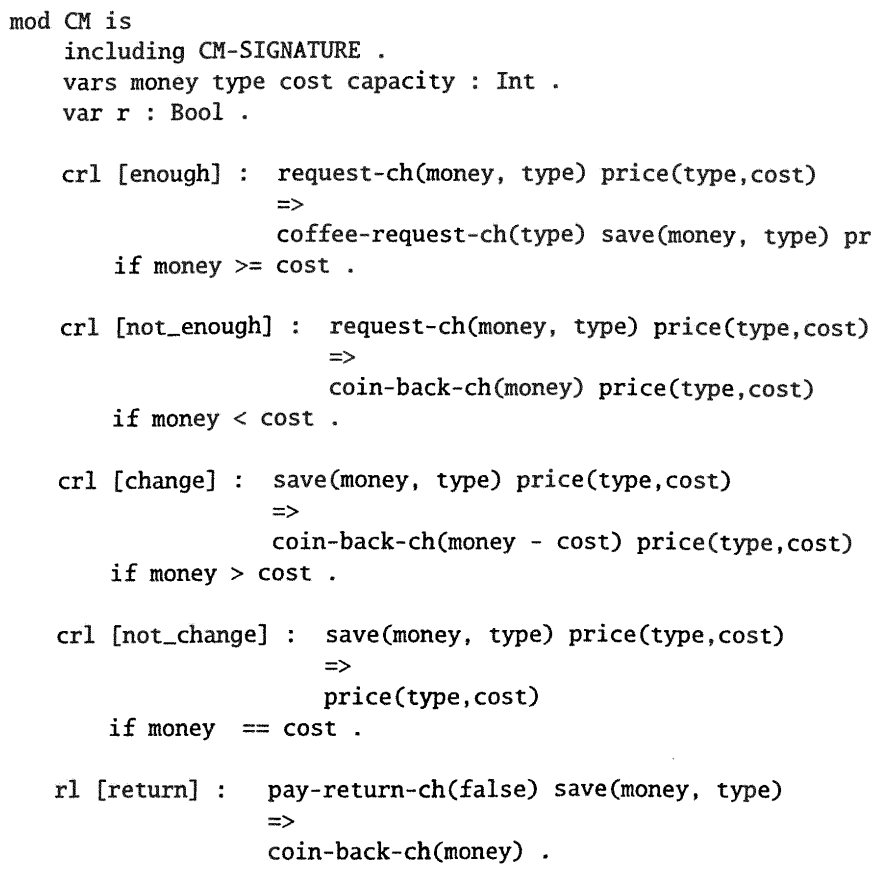

crl [consume] : coffee-request-bf(type) ready-bf(true) storage(type, capacity) $\Rightarrow$

rl [input] : money-cmi(money) coffeetype-cmi(type)

$\Rightarrow$

request-cmi (money, type) sig(true).

rl [take_cup] : sig(true)

$\Rightarrow$

ready-cmi (true).

rl [back_coin] : coin-back-cmi (money)

$\Rightarrow$

change-cmi (money). 


$$
\begin{aligned}
\mathrm{rl} \text { [send_coffee] : } & \text { coffee-serve-cmi(type) } \\
& \Rightarrow \\
& \text { ready-enjoy-cmi(type). }
\end{aligned}
$$

rl [t1] : request-cmi (money, type) $\Rightarrow$ request-ch(money, type) .

rl [t2] : coin-back-ch (money) $\Rightarrow$ coin-back-cmi (money).

rl [t3] : coffee-request-ch(type) $\Rightarrow$ coffee-request-bf(type).

rl [t4]: pay-return-bf(r) $\Rightarrow$ pay-return-ch( $r)$.

rl [t5] : $\operatorname{ready-cmi(r)\Rightarrow ~ready-bf(r).~}$

rl [t6] : coffee-serve-bf(type) $\Rightarrow$ coffee-serve-cmi(type).

endm

The following is the atomic predicates of coffee machine example demonstrated in the Chapter 8:

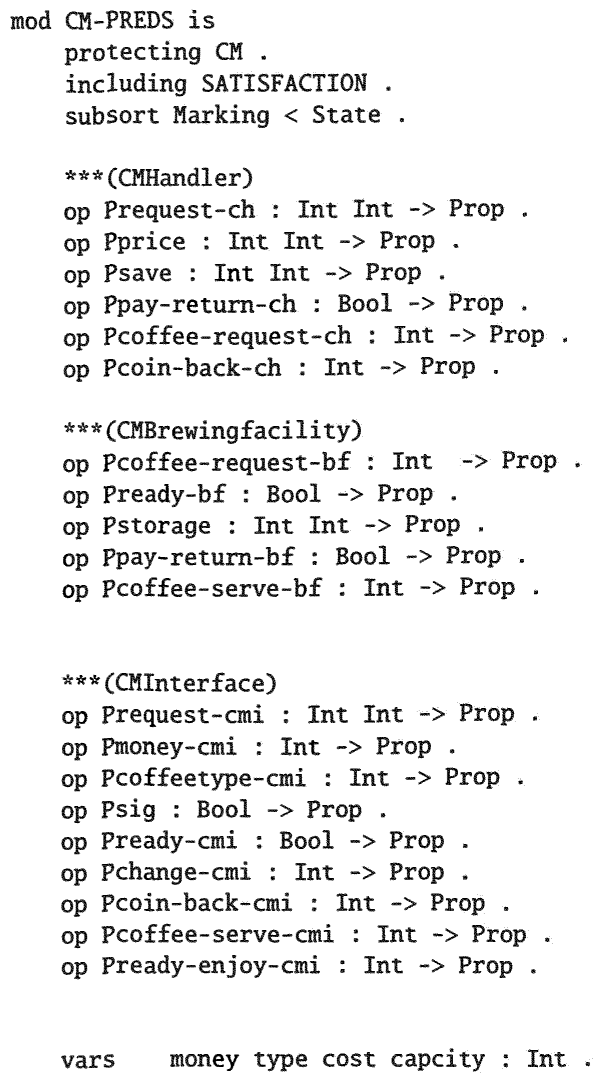


var bool : Bool.

vars $\mathrm{m} 1 \mathrm{~m} 2$ : Marking.

eq $\mathrm{m} 1$ request-ch(money, type) $\mathrm{m} 2 \quad \mid=$ Prequest $-\mathrm{ch}$ (money, type) $=$ true .

eq $\mathrm{m} 1$ price (type, cost) $\mathrm{m} 2 \mathrm{I}=$ Pprice (type, cost) $=$ true .

eq $\mathrm{m} 1$ save (money, type) $\mathrm{m} 2 \quad$ I= Psave (money, type) $=$ true .

eq $\mathrm{m} 1$ pay-return-ch(bool) $\mathrm{m} 2 \quad \mathrm{I}=$ Ppay-return-ch(bool) = true .

eq $\mathrm{m} 1$ coffee-request-ch(type) $\mathrm{m} 2 \quad \mid=$ Pcoffee-request-ch(type) $=$ true .

eq $\mathrm{m} 1$ coin-back-ch(money) $\mathrm{m} 2 \mathrm{l}=$ Pcoin-back-ch(money) $=$ true .

eq $\mathrm{m} 1$ coffee-request-bf(type) $\mathrm{m} 2 \quad \mid=$ Pcoffee-request-bf(type) $=$ true .

eq $\mathrm{m} 1$ ready-bf(bool) $\mathrm{m} 2 \quad \mathrm{l}=$ Pready-bf(bool) = true .

eq $\mathrm{m} 1$ storage (type, capcity) $\mathrm{m} 2 \mathrm{l}=$ Pstorage (type, capcity) = true .

eq $\mathrm{m} 1$ pay-return-bf(bool) $\mathrm{m} 2 \mathrm{I}=$ Ppay-return-bf(bool) $=$ true .

eq $\mathrm{m} 1$ coffee-serve-bf(type) $\mathrm{m} 2 \quad \mid=$ Pcoffee-serve-bf(type) $=$ true .

eq $\mathrm{m} 1$ request-cmi (money, type) $\mathrm{m} 2 \mathrm{I}=$ Prequest-cmi (money, type $)=$ true . eq $\mathrm{m} 1$ money-cmi(money) $\mathrm{m} 2 \quad \mathrm{I}=$ Pmoney-cmi (money) $=$ true .

eq $\mathrm{m} 1$ coffeetype-cmi (type) $\mathrm{m} 2 \mathrm{I}=$ Pcoffeetype-cmi (type) $=$ true .

eq $\mathrm{m} 1 \mathrm{sig}$ (bool) $\mathrm{m} 2 \mathrm{I}=$ Psig(bool) = true .

eq $\mathrm{m} 1$ change-cmi (money) $\mathrm{m} 2 \quad \mid=$ Pchange-cmi (money) = true .

eq $\mathrm{m} 1$ coin-back-cmi (money) $\mathrm{m} 2 \mathrm{I}=$ Pcoin-back-cmi (money) $=$ true .

eq $\mathrm{m} 1$ coffee-serve-cmi (type) $\mathrm{m} 2 \quad \mathrm{I}=$ Pcoffee-serve-cmi(type) $=$ true .

eq $\mathrm{m} 1$ ready-enjoy-cmi(type) $\mathrm{m} 2 \mathrm{I}=$ Pready-enjoy-cmi (type) $=$ true .

eq $\mathrm{m} 1$ ready-cmi(bool) $\mathrm{m} 2 \mathrm{I}=$ Pready $-\mathrm{cmi}($ bool $)=$ true .

endm 


\section{APPENDIX B}

\section{MAUDE CODE FOR ONLINE SHOPPING}

The following is the functional module for online shopping system demonstrated in Chapter 8:

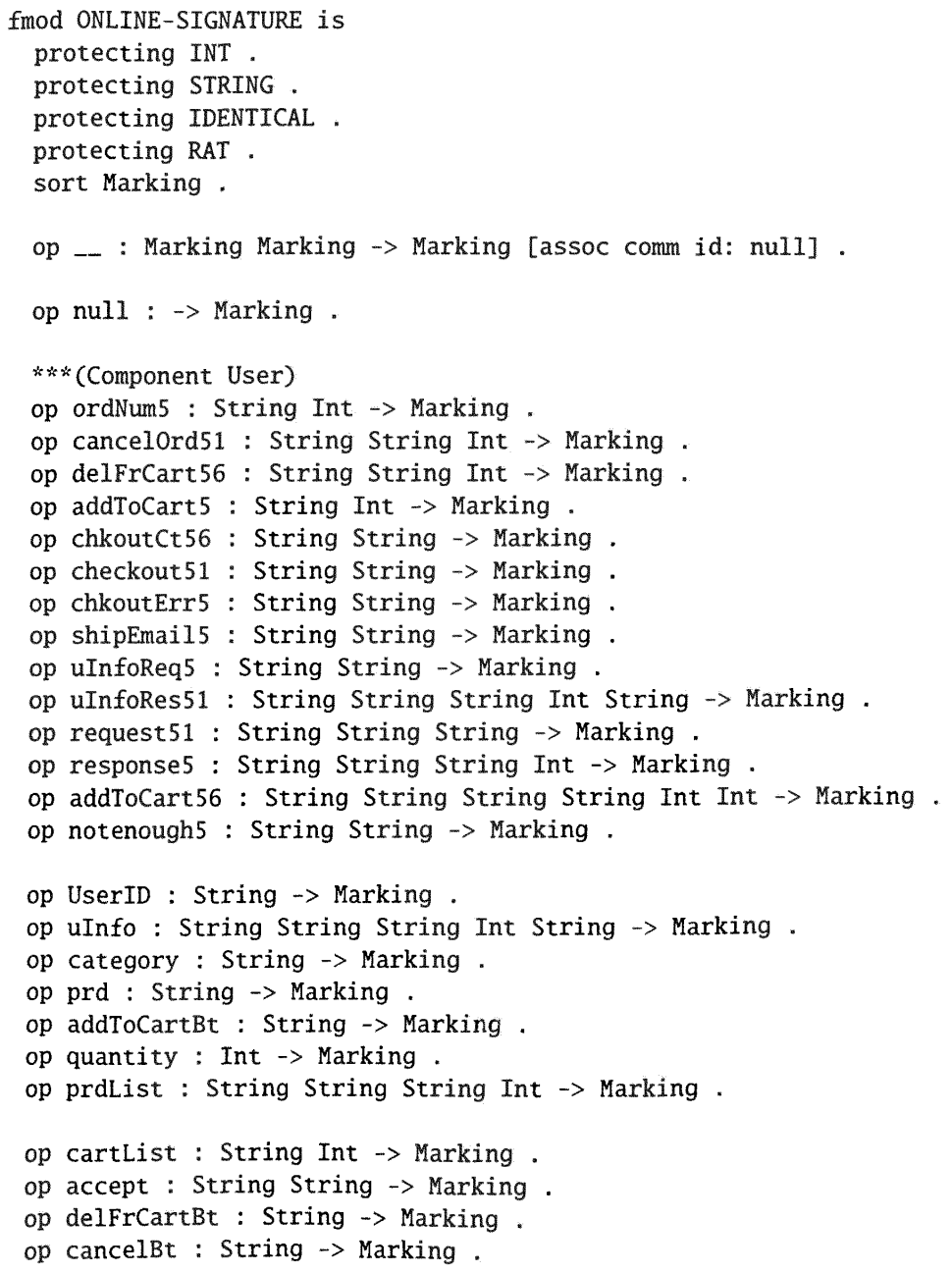




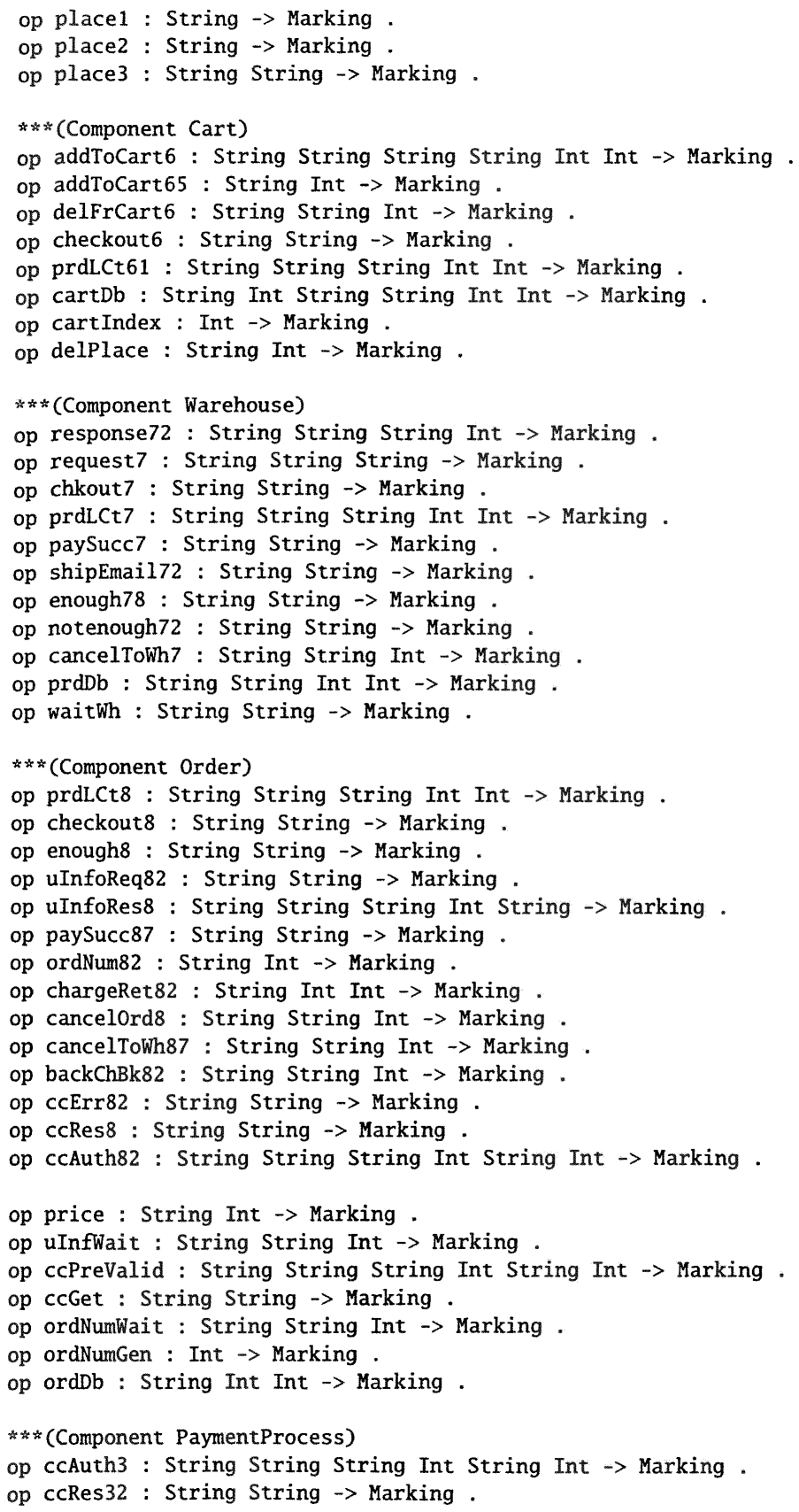


op backChCc3 : String String Int $\rightarrow$ Marking .

op ccAuthor : String String Int String Int String $\rightarrow$ Marking .

op cStolen : String String String $\rightarrow$ Marking .

op coutlmt : String String String $\rightarrow$ Marking .

op cExp : String String String $\rightarrow$ Marking .

op cPass : String String String $\rightarrow$ Marking .

$* * *$ (Component RetailerBank)

op backChBk4 : String String Int -> Marking .

op chargeRet4 : String Int Int $\rightarrow$ Marking .

op backChCc43 : String String Int $\rightarrow$ Marking .

op retailerBk : String Int Int $\rightarrow$ Marking .

end $f m$

The following is the system module for online shopping system demonstrated in Chapter 8:

mod ONLINE is

including ONLINE-SIGNATURE .

vars uid1 uid2 uid3 cat ccExp name pname ccNum $f \mathrm{fl} f 2 \mathrm{k}$ ru $\mathrm{rp} d$ ac kc : String .

vars cat1 cat2 eg name1 name2 ccNum1 ccNum2 ccExp1 ccExp2 ccStatus : String .

vars ordNm ordNm1 ordNm2 index limit price q mindex q1 q2 limit1 limit2 balance : Int .

vars price1 price2 : Int .

vars $b$ : Bool.

$* * *$ (Component User)

rl [t5_6] : ordNum5 (uid1, ordNm) $\Rightarrow$ orderList(uid1, ordNm) .

rl [t5_12] : cancelBt $(\mathrm{f} 2) \Rightarrow \operatorname{place2}(\mathrm{f} 2)$.

rl [t5_11] : delFrCartBt $(f 1) \Rightarrow \operatorname{place1}(f 1)$.

rl [t5_9] : accept (uid1,"checkout") $\Rightarrow$ place3(uid1,"checkout") .

rl [t5_1] : response5 (uid1, pname, cat,price) $\Rightarrow$ prdList (uid1, pname, cat,price) .

rl [t5_3] : addToCart5 (uid1, index) $\Rightarrow$ cartlist(uid1,index) .

rl [t5_0] : UserID(uid1) category (cat) $\Rightarrow$ request51(uid1, "pReq", cat) UserID(uid1) .

rl [t5_10] : cartList(uid1, index) $\Rightarrow$ accept (uid1, "checkout") .

crl [t5_5] : UserID(uid1) cancelBt(f2) orderList (uid2, ordNm)

$\Rightarrow$

cancelord51 (uid1, $f 2$, ordNm) UserID(uid1)

if $\mathrm{f} 2==$ "cancel" and uid1 $==$ uid2 .

crl [t5_4] : UserID(uid1) delFrCartBt(f1) cartList (uid2, index)

$\Rightarrow$

delFrCart56(uid2,f1, index) UserID(uid1)

if $\mathrm{f} 1===$ "del" and uid1 $===$ uid2 .

crl [t5_8]: UserID(uid1) accept (uid2,k)

$\Rightarrow$

checkout51(uid2,k) chkoutCt56(uid2,k) UserID(uid1)

if $\mathrm{k}===$ "checkout" and uid1 $===$ uid2 .

cr1 [t5_7] : uInfoReq5(uid1,ru) uInfo(uid2, name, ccNum, limit, ccExp) 
$\Rightarrow$

uInfoRes51 (uid2, name, ccNum, limit, ccExp)

if uid $1===$ uid2 and ru === "uReq" .

crl [t5_2] : UserID(uid1) prd(pname) addToCartBt(f) quantity(q)

prdList (uid2, pname, cat,price)

$\Rightarrow$

addToCart56(uid2,f,pname, cat,price,q) UserID(uid1)

if uid1 === uid2 .

$* * *$ (Component Cart)

crl [t6_1] : addToCart6 (uid1,ac,pname, cat,price,q) cartIndex (mindex)

$\Rightarrow$

cartIndex (mindex +1 ) cartDb (uid1, mindex, pname, cat,price, $q$ )

addToCart65 (uid1, mindex)

if $\mathrm{ac}==$ "addCart" .

crl [t6_2] : cartDb(uid1, mindex, pname, cat,price,q) delFrCart6(uid2,d,mindex)

$\Rightarrow$

delplace (uid2, mindex)

if uid1 $===$ uid2 and $d===$ "del" .

crl [t6_3] : cartDb(uid1,mindex, pname,cat,price,q) checkout6(uid2,kc)

$\Rightarrow$

prdLCt61 (uid2, pname, cat,price, q)

if uid1 === uid2 and $\mathrm{kc}===$ "checkout" .

$* * *$ (Component Warehouse)

cr1 [t7_Q] : request7(uid1,f,cat1) prdDb(pname,cat2,price,q)

$\Rightarrow$

response 72(uid1, pname, cat1,price) prdDb(pname, cat2,price, q)

if cat $1==$ cat 2 and $f==$ "pReq".

crl [t7_10] : chkout7(uid1,f) prdLCt7(uid2,pname,cat1,price,q1)

prdDb (pname, cat2, price, q2)

$\Rightarrow$

enough78 (uid1, "enough") waitWh(uid1, "wait")

$\operatorname{prdDb}$ (pname, cat2, price, q2 - q1)

if (uid1 $===$ uid2 and cat $1===\operatorname{cat} 2$ and $q 2>$ q1).

crl [t7_11] : chkout7(uid1,f) prdLCt7(uid2,pname,cat1,price,q1)

prdDb (pname, cat2, price, q2)

$\Rightarrow$

notenough72 (uid1, "notenough") prdDb(pname, cat2, price, q2)

if (uid1 $===$ uid2 and cat $1===$ cat 2 and $q 1>$ q2).

crl [t7_2] : paySucc7(uid1,f1) waitWh(uid2,f2)

$\Rightarrow$

shipEmail72 (uid1, "shipEmail")

if uid1 $===$ uid2 and $\mathrm{f1}===$ "paySucc" and $\mathrm{f2}===$ "wait" .

$* * *$ (Component Order)

crl [t8_o] : prdLCt8(uid1,pname,cat,price,q) checkout8(uid2,f1) enough8(uid3,f2)

$\Rightarrow$ price(uid2,price)

if $\mathrm{f} 1===$ "checkout" and $\mathrm{f} 2===$ "enough" and

uid1 $===$ uid2 and uid $1===$ uid3 .

crl [t8_2] : uInfWait (uid1,f1,price) uInfoRes8 (uid2, name, ccNum, limit, ccExp)

$\Rightarrow$

ccPreValid(uid1, name, ccNum, limit, ccExp,price) 
ccAuth82 (uid1, name, ccNum, limit, ccExp, price)

if uid2 = = uid1.

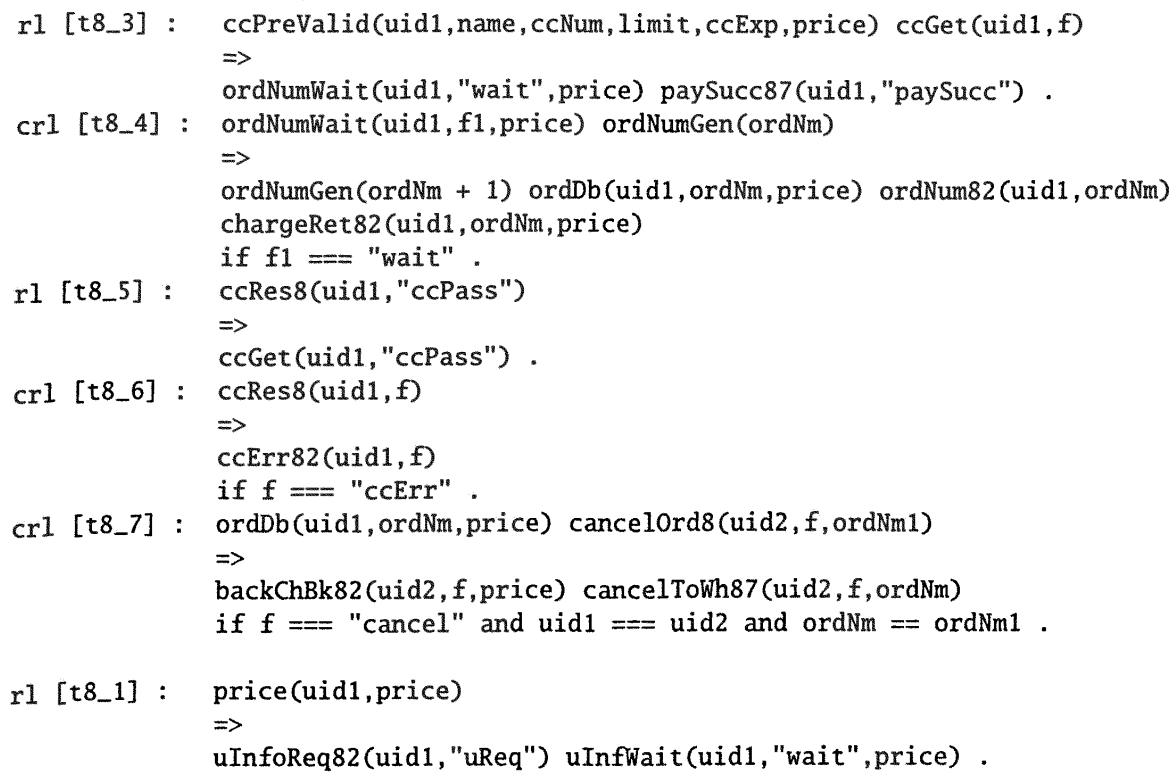




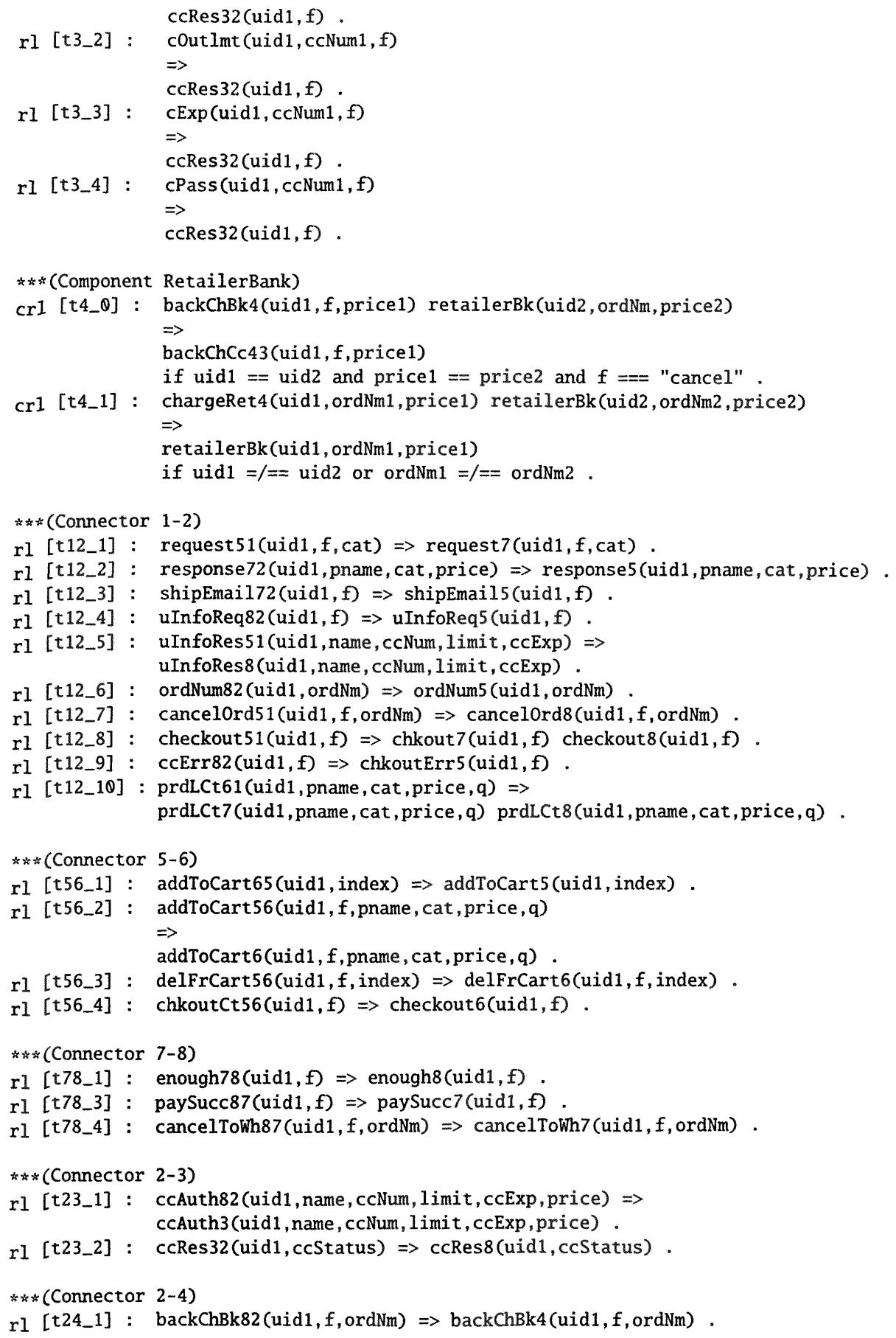


rl [t24_2] : chargeRet82(uid1,ordNm,price) $\Rightarrow$ chargeRet4(uid1, ordNm,price) .

$* * *$ (Connector 3-4)

rl [t34_1] : backChCc43(uid1,f,price) $\Rightarrow$ backChCc3(uid1,f,price) .

endm

The following is the predicate module of online shopping system demonstrated in the Chapter 8:

$\bmod$ ONLINE-PREDS is

protecting ONLINE .

including SATISFACTION .

subsort Marking < State.

$* * *$ (Component User)

op PordNum5 : String $\rightarrow$ Prop.

op Pcancelord51 : String String Int $\rightarrow$ Prop .

op PdelFrCart56 : String String Int $\rightarrow$ Prop .

op PaddToCart5 : String Int $\rightarrow$ Prop .

op PchkoutCt56 : String String $\rightarrow$ Prop .

op Pcheckout51 : String String $\rightarrow$ Prop .

op PchkoutErr5 : String String $\rightarrow$ Prop .

op PshipEmail5 : String String $\rightarrow$ Prop .

op PuInfoReq5 : String String $\rightarrow$ Prop .

op PuInfoRes51 : String String String Int String $\rightarrow$ Prop .

op Prequest51 : String String String $\rightarrow$ Prop .

op Presponse5 : String String $\rightarrow$ Prop .

op PaddToCart56 : String String String $\rightarrow$ Prop .

op Pnotenough5 : String String $\rightarrow$ Prop .

op PUserID : String $\rightarrow$ Prop .

op PuInfo : String String String Int String $\rightarrow$ Prop .

op Pcategory : String $\rightarrow$ Prop .

op Pprd : String $\rightarrow$ Prop .

op PaddToCartBt : String $\rightarrow$ Prop .

op Pquantity : Int $\rightarrow$ Prop .

op PprdList : String String String Int $\rightarrow$ Prop .

op Pcartlist : String Int $\rightarrow$ Prop .

op Paccept : String String $\rightarrow$ Prop .

op PdelFrCartBt : String $\rightarrow$ Prop .

op PcancelBt : String $\rightarrow$ Prop .

op PorderList : String Int $\rightarrow$ Prop .

op Pplace1 : String $\rightarrow$ Prop .

op Pplace2 : String $\rightarrow$ Prop .

op Pplace3 : String String $\rightarrow$ Prop .

*** (Component Cart)

op PaddToCart6 : String String String String Int Int $\rightarrow$ Prop . 


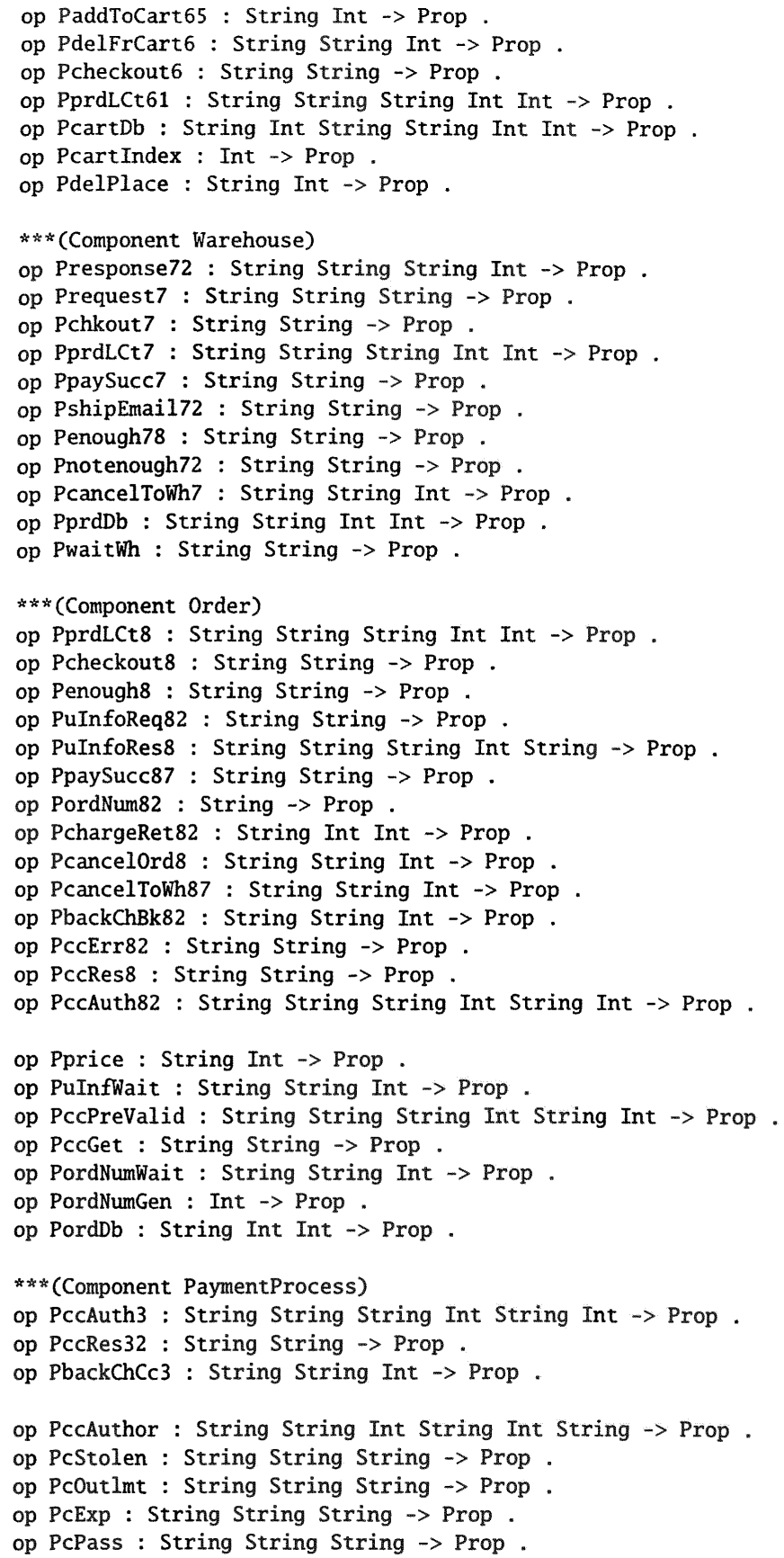




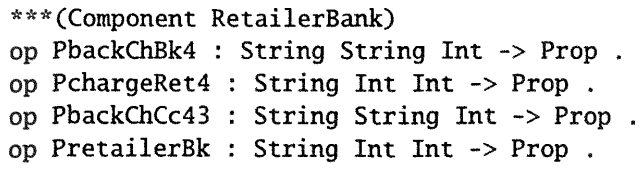

vars uid1 uid2 uid3 cat ccExp name pname ccNum $f \mathrm{f1} f 2 \mathrm{k}$ ru $\mathrm{rp} d$ ac kc : String . vars cat 1 cat 2 eg name1 name 2 ccNum1 ccNum2 ccExp1 ccExp2 ccStatus : String .

vars ordNm ordNm1 ordNm2 index limit price q mindex q1 q2 limit1 limit2 balance : Int .

vars price1 price2 : Int .

vars $\mathrm{m} 1 \mathrm{~m} 2$ : Marking.

$* * *$ (Component User)

eq $\mathrm{m} 1$ ordNum5 (uid1, ordNm) $\mathrm{m} 2 \quad \mid=\operatorname{PordNum} 5$ (uid1) $=$ true .

eq $\mathrm{m} 1$ cancelord51(uid1,f, ordNm) $\mathrm{m} 2 \quad \mid=$ Pcancelord51(uid1, $f$, ordNm) $=$ true .

eq $\mathrm{m} 1$ delFrCart56(uid1, $f$, index) $\mathrm{m} 2 \quad \mid=$ PdelFrCart56(uid1, $f$, index) = true .

eq $\mathrm{m} 1$ addToCart5 (uid1, index) $\mathrm{m} 2 \quad \mid=$ PaddToCart5(uid1, index) = true .

eq $\mathrm{m} 1$ chkoutCt56(uid1,k) m2 $\mathrm{I}=$ PchkoutCt56(uid1,k) = true .

eq $\mathrm{m} 1$ checkout 51 (uid1,k) $\mathrm{m} 2 \quad \mathrm{l}=$ Pcheckout51(uid1,k) = true .

eq $\mathrm{m} 1$ chkoutErr5 (uid1, $f$ ) $\mathrm{m} 2 \quad \mid=$ PchkoutErr5(uid1, $f$ ) $=$ true .

eq $\mathrm{m} 1$ shipEmail5(uid1,f) $\mathrm{m} 2 \quad \mid=$ PshipEmail5(uid1,f) $=$ true.

eq $\mathrm{m} 1$ uInfoReq5(uid1, $f$ ) $\mathrm{m} 2 \quad \mid=$ PuInfoReq5(uid1, $f$ ) $=$ true .

eq $\mathrm{m} 1$ uInfoRes51(uid1, name, ccNum, limit, ccExp) $\mathrm{m} 2$ I= PuInfoRes51(uid1, name, ccNum, limit, ccExp) = true .

eq $m 1$ request 51 (uid1, $f, c a t$ ) $m 2 \quad l=$ Prequest 51 (uid $1, f$, cat) $=$ true .

eq $\mathrm{m} 1$ response 5 (uid1, pname, cat,price) $\mathrm{m} 2 \quad \mid=$ Presponse 5 (uid1, cat) = true .

eq $\mathrm{m} 1$ addToCart56(uid1, f, pname,cat,price, q) $\mathrm{m} 2 \mathrm{l}=$ PaddToCart56(uid1, $f$, cat) $=$ true . eq $\mathrm{m} 1$ notenough5 (uid1, $f$ ) $\mathrm{m} 2 \quad \mid=$ Pnotenough5 (uid $1, f$ ) $=$ true .

eq $\mathrm{m} 1$ UserID(uid1) $\mathrm{m} 2 \quad \mid=$ PUserID(uid1) = true .

eq $\mathrm{m} 1$ uInfo(uid1, name, ccNum, limit, ccExp) $\mathrm{m} 2 \quad$ I=

PuInfo(uid1, name, ccNum, limit, ccExp) = true .

eq $\mathrm{m} 1$ category (cat) $\mathrm{m} 2 \quad \mathrm{l}=$ Pcategory $(\mathrm{cat})=$ true .

eq $\mathrm{m} 1$ prd(name) $\mathrm{m} 2 \mathrm{I}=\operatorname{Pprd}($ name $)=$ true .

eq $m 1$ addToCartBt $(f) m 2 \quad \mid=$ PaddToCartBt $(f)=$ true.

eq $m 1$ quantity $(q) m 2 \quad \mid=$ Pquantity $(q)=$ true .

eq $\mathrm{m} 1$ prdList(uid1, pname, cat,price) $\mathrm{m} 2 \quad \mid=$ PprdList (uid1, pname, cat,price) $=$ true .

eq $\mathrm{m} 1$ cartList(uid1, index) $\mathrm{m} 2 \quad \mid=$ PcartList(uid1, index) $=$ true .

eq $m 1$ accept(uid1,f) $m 2 \quad \mid=$ Paccept(uid1, $f$ ) = true.

eq $m 1 \operatorname{delFrCartBt}(f) \mathrm{m} 2 \quad \mid=\operatorname{PdelFrCartBt}(f)=$ true .

eq $m 1$ cancelBt $(f) \mathrm{m} 2 \quad \mid=$ PcancelBt $(f)=$ true .

eq $\mathrm{m} 1$ orderList (uid1, ordNm) $\mathrm{m} 2 \mathrm{l}=$ PorderList(uid1, ordNm) $=$ true .

eq $m 1 \operatorname{place} 1(f) m 2 \quad \mid=\operatorname{Pplace} 1(f)=$ true .

eq $m 1$ place2 $(f) \mathrm{m} 2 \quad \mid=\operatorname{Pplace} 2(f)=$ true .

eq $\mathrm{m} 1$ place3(uid1,f) $\mathrm{m} 2 \quad \mid=\operatorname{Pplace} 3(\mathrm{uid} 1, f)=$ true .

**** (Component Cart)

eq $\mathrm{m} 1$ addToCart6(uid1, $f$, pname, cat,price, $q$ ) $\mathrm{m} 2 \quad \mid=$ PaddToCart6 (uid1, $f$, pname, cat, price, $q$ ) $=$ true .

eq $\mathrm{m} 1$ addToCart65(uid1, index) $\mathrm{m} 2 \mathrm{I}=$ PaddToCart65(uid1, index) = true . 


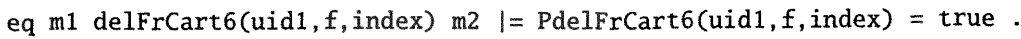

eq $m 1$ checkout 6 (uid1, $f$ ) $m 2 \quad I=$ Pcheckout6(uid1, $f$ ) $=$ true .

eq $\mathrm{m} 1$ prdLCt61(uid1, pname, cat, price, q) $\mathrm{m} 2$ । =

PprdLCt61(uid1, pname, cat, price, $q$ ) = true .

eq $\mathrm{m} 1$ cartDb(uid1, index, pname, cat,price, q) $\mathrm{m} 2 \quad \mathrm{I}=$

PcartDb (uid1, index, pname, cat, price, $q)=$ true .

eq $\mathrm{m} 1$ cartIndex (index) $\mathrm{m} 2 \quad \mid=$ PcartIndex(index) = true .

eq $\mathrm{m} 1$ delPlace(uid1, index) $\mathrm{m} 2 \quad \mathrm{I}=\mathrm{Pdel}$ Place(uid1, index) $=$ true .

*** (Component Warehouse)

eq $\mathrm{m} 1$ response 72 (uid1, pname, cat,price) $\mathrm{m} 2 \quad \mid=$

Presponse72 (uid1, pname, cat,price) = true .

eq $m 1$ request 7 (uid $1, f, c a t$ ) $m 2 \quad \mid=$ Prequest 7 (uid $1, f, c a t$ ) $=$ true .

eq $\mathrm{m} 1$ chkout7 (uid1, $f$ ) $\mathrm{m} 2 \quad \mid=\operatorname{Pchkout} 7$ (uid1, $f$ ) $=$ true .

eq $\mathrm{m} 1$ prdLCt7 (uid1, pname, cat, price, q) $\mathrm{m} 2$ l=

PprdLCt7 (uid1, pname, cat,price, $q$ ) = true .

eq $m 1$ paySucc7(uid1,f) $m 2 \quad I=$ PpaySucc7(uid1,f) $=$ true .

eq $m 1$ shipEmail72(uid1, $f$ ) $m 2 \quad \mid=$ PshipEmail72(uid1, $f$ ) $=$ true .

eq $m 1$ enough78(uid1, $f$ ) $m 2 \quad I=$ Penough78(uid1, $f$ ) $=$ true .

eq $\mathrm{m} 1$ notenough72(uid1, $f$ ) $\mathrm{m} 2 \mathrm{I}=$ Pnotenough72(uid1, $f$ ) $=$ true.

eq $\mathrm{m} 1$ cancelToWh7 (uid1, $f$, ordNm) $\mathrm{m} 2 \quad \mid=$ PcancelToWh7 (uid1, $f$, ordNm) $=$ true .

eq $\mathrm{m} 1 \mathrm{prdDb}$ (pname, cat, price, q) $\mathrm{m} 2 \mathrm{l}=\operatorname{PprdDb}$ (pname, cat, price, $q$ ) $=$ true .

eq $m 1$ waitWh(uid1,f) $m 2 \quad \mid=$ PwaitWh(uid1, $f$ ) = true .

*** (Component Order)

eq $\mathrm{m} 1$ prdLCt8(uid1, pname, cat,price, q) m2 I=

PprdLCt8 (uid1, pname, cat,price, $q$ ) = true .

eq $m 1$ checkout 8 (uid1,f) $m 2 \quad \mid=$ Pcheckout8 (uid1, $f$ ) $=$ true.

eq $\mathrm{m} 1$ enough8(uid1, $f$ ) $\mathrm{m} 2 \mid=$ Penough8(uid $1, f$ ) $=$ true .

eq $\mathrm{m} 1$ uInfoReq82(uid1, $f$ ) $\mathrm{m} 2 \quad \mid=$ PuInfoReq82(uid1, $f$ ) $=$ true .

eq m1 uInfoRes8 (uid1, name, ccNum, limit, ccExp) m2 l=

PuInfoRes8 (uid1, name, ccNum, limit, ccExp) = true .

eq $\mathrm{m} 1$ paySucc87(uid1,f) $\mathrm{m} 2 \quad$ I= PpaySucc87(uid1, $f$ ) $=$ true .

eq $\mathrm{m} 1$ ordNum82(uid1, ordNm) $\mathrm{m} 2 \quad \mid=$ PordNum82(uid1) = true.

eq $\mathrm{m} 1$ chargeRet 82 (uid1, ordNm, price) $\mathrm{m} 2 \quad \mid=$ PchargeRet 82 (uid1, ordNm, price) = true .

eq $m 1$ cancelord8 (uid1, $f$, ordNm) $m 2 \quad I=$ Pcancelord8 (uid1, $f$, ordNm) $=$ true .

eq $m 1$ cancelToWh87 (uid1, $f$, ordNm) $m 2 \quad I=$ PcancelToWh87 (uid1, $f$, ordNm) $=$ true .

eq $m 1$ backChBk82 (uid1, $f$,price) $m 2 \quad l=$ PbackChBk82(uid1, $f$,price) $=$ true .

eq $m 1 \operatorname{ccErr} 82$ (uid1, $f$ ) $\mathrm{m} 2 \quad \mid=\operatorname{PccErr} 82$ (uid1, $f$ ) $=$ true .

eq $\mathrm{m} 1$ ccRes8 (uid1, $f$ ) $\mathrm{m} 2 \quad \mid=$ PccRes8 (uid1, $f$ ) = true .

eq $\mathrm{m} 1$ ccAuth82(uid1, name,ccNum, limit,ccExp,price) $\mathrm{m} 2 \mathrm{l=}$

PccAuth82(uid1, name, ccNum, limit, ccExp,price) = true .

eq $\mathrm{m} 1$ price(uid1,price) $\mathrm{m} 2 \quad \mathrm{I}=$ Pprice(uid1,price) $=$ true .

eq $\mathrm{m} 1$ uInfWait(uid1,f,price) $\mathrm{m} 2 \mathrm{l}=$ PuInfWait(uid1,f,price) = true .

eq $\mathrm{m} 1 \mathrm{ccPreValid}($ uid1, name, ccNum, limit, ccExp,price) $\mathrm{m} 2 \mathrm{l}=$

PccPreValid(uid1, name, ccNum, limit, ccExp,price) $=$ true .

eq $\mathrm{m} 1 \mathrm{ccGet}$ (uid1, f) $\mathrm{m} 2 \quad \mid=$ PccGet(uid1, $f$ ) = true .

eq $m 1$ ordNumWait (uid1,f,price) $m 2 \quad l=$ PordNumWait(uid1,f,price) = true .

eq $\mathrm{m} 1$ ordNumGen (ordNm) $\mathrm{m} 2 \mathrm{l}=$ PordNumGen (ordNm) = true.

eq $\mathrm{m} 1$ ordDb(uid1, ordNm, price) $\mathrm{m} 2 \mathrm{l}=$ PordDb(uid1, ordNm, price) $=$ true .

$* * *$ (Component PaymentProcess)

eq $\mathrm{m} 1$ ccAuth3(uid1, name, ccNum, limit, ccExp, price) $\mathrm{m} 2 \mathrm{|}=$

PccAuth3 (uid1, name, ccNum, limit, ccExp, price) = true . 
eq $m 1 \operatorname{ccRes} 32$ (uid1, $f$ ) $m 2 \quad \mid=$ PccRes32(uid1, $f$ ) = true .

eq $m 1$ backChCc3(uid1,f,price) $m 2 \quad=$ PbackChCc3(uid1, f,price) = true .

eq $\mathrm{m} 1$ ccAuthor (name, ccNum, limit, ccExp, balance, ccStatus) $\mathrm{m} 2 \mathrm{I}=$ PccAuthor (name, ccNum, limit, ccExp, balance, ccStatus) = true .

eq $\mathrm{m} 1$ cStolen(uid1, ccNum, ccStatus) $\mathrm{m} 2 \quad \mid=$ PcStolen(uid1, ccNum, ccStatus) = true .

eq $\mathrm{m} 1$ coutlmt (uid1, ccNum, ccStatus) $\mathrm{m} 2 \mathrm{I}=$ PcOutlmt (uid1, ccNum, ccStatus) = true .

eq $\mathrm{m} 1$ cExp(uid1, ccNum, ccStatus) $\mathrm{m} 2 \quad \mid=$ PcExp (uid1, ccNum, ccStatus) = true .

eq $\mathrm{m} 1 \mathrm{cPass}$ (uid1, ccNum, ccStatus) $\mathrm{m} 2 \mathrm{I}=\mathrm{PcPass}$ (uid1, ccNum, ccStatus) = true .

$* *$ (Component RetailerBank)

eq $m 1$ backChBk4(uid1,f,price) $m 2 \mid=$ PbackChBk4(uid1,f,price) = true .

eq $m 1$ chargeRet4 (uid1, ordNm, price) $m 21=$ PchargeRet4 (uid1, ordNm, price) = true .

eq $m 1$ backChCc43 (uid1,f,price) $m 2 \quad I=$ PbackChCc43(uid1,f,price) = true .

eq $\mathrm{m} 1$ retailerBk(uid1, ordNm, price) $\mathrm{m} 2 \mathrm{I}=$ PretailerBk(uid1, ordNm,price) $=$ true .

endm 


\section{APPENDIX C \\ RUNTIME CODE FOR COFFEE MACHINE}

The generated code for the coffee machine contains hundreds of files, we cannot list every

file due to space limit. Following the ArchJava code for the component CoffeeMachine.

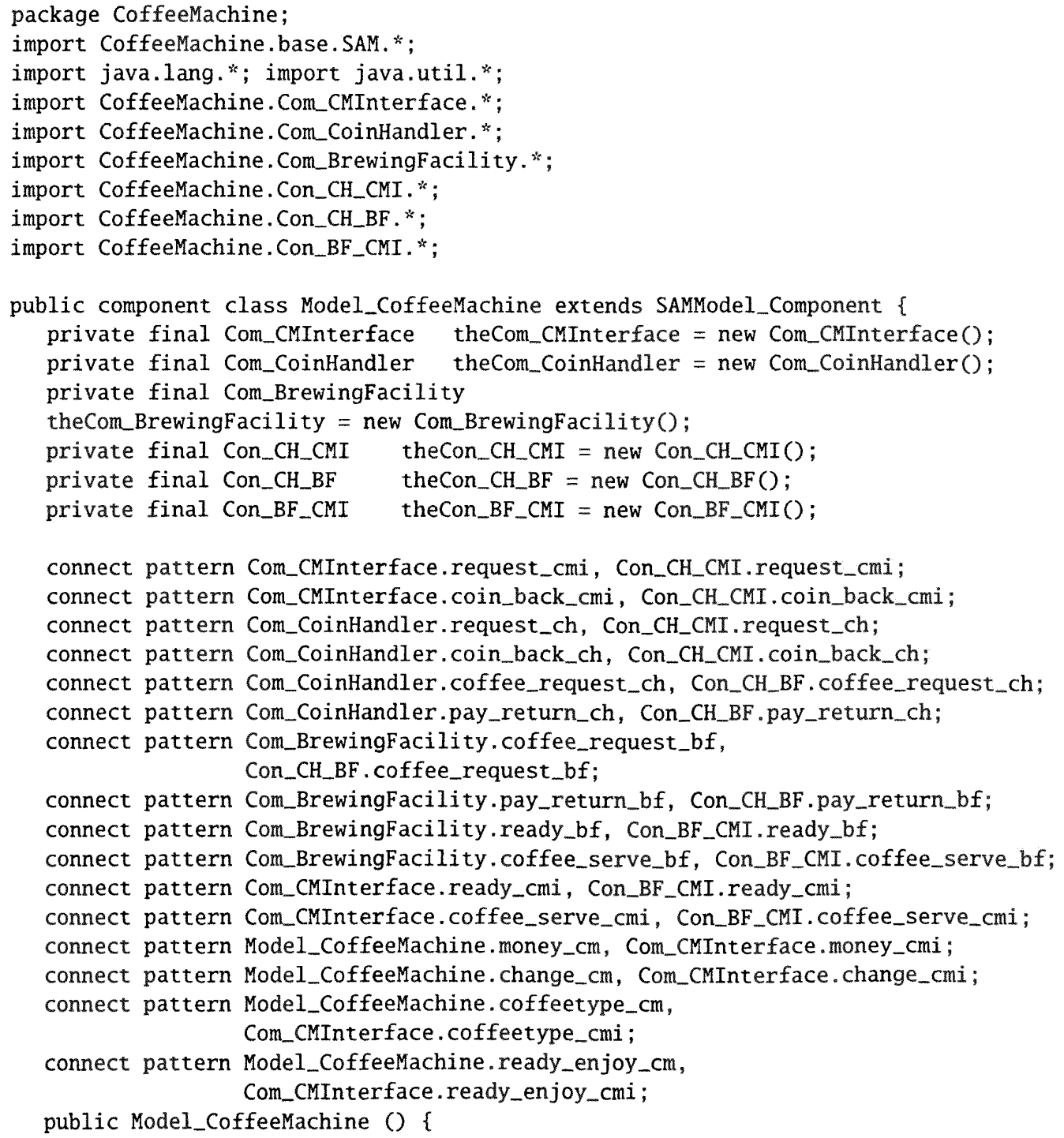




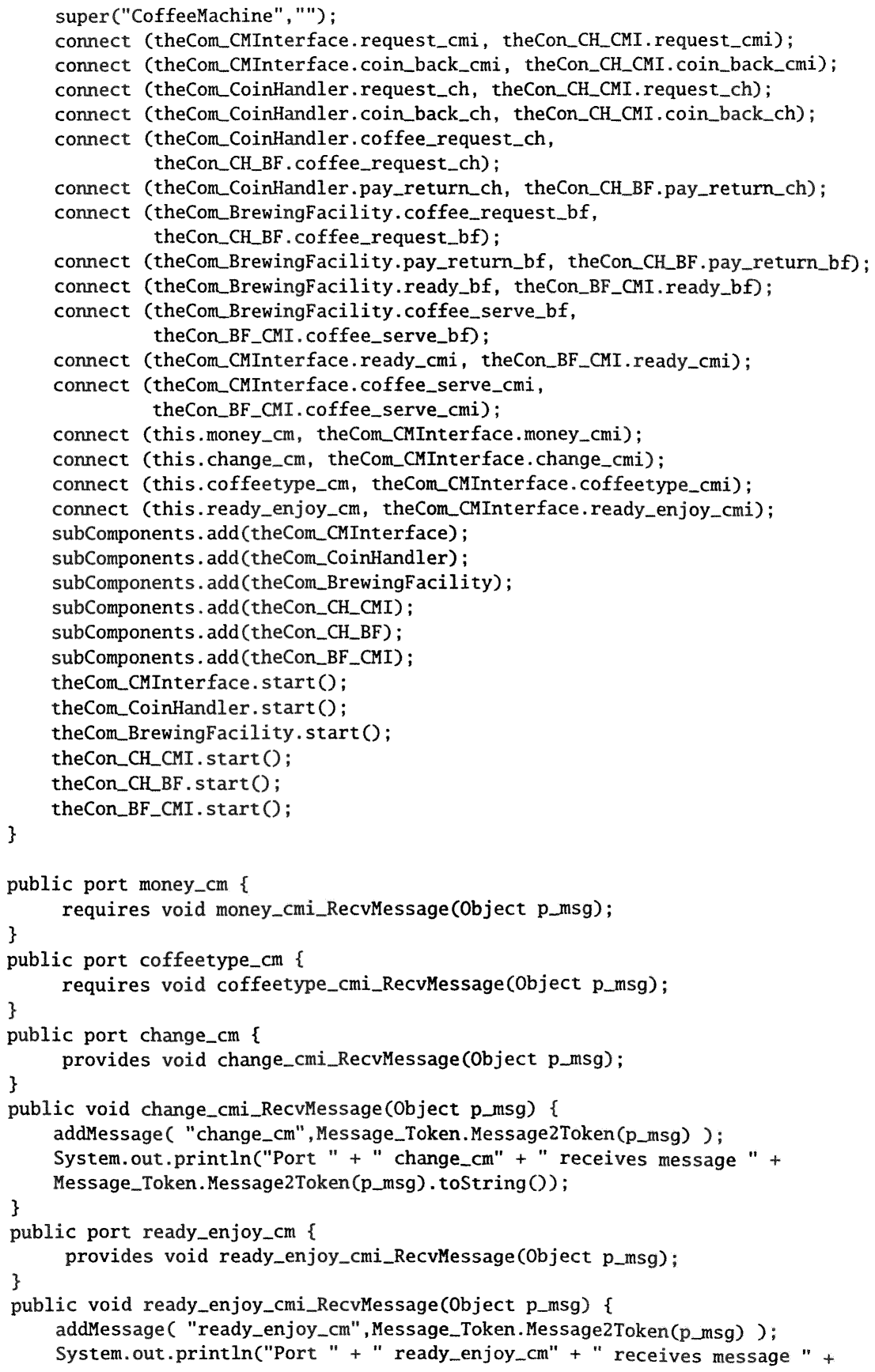




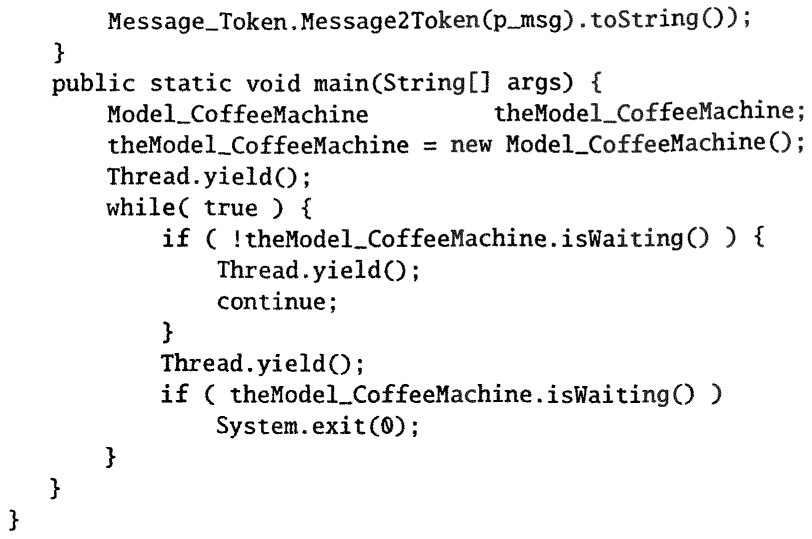

The generated aspectj code for the component CoffeeMachine is shown in the following.

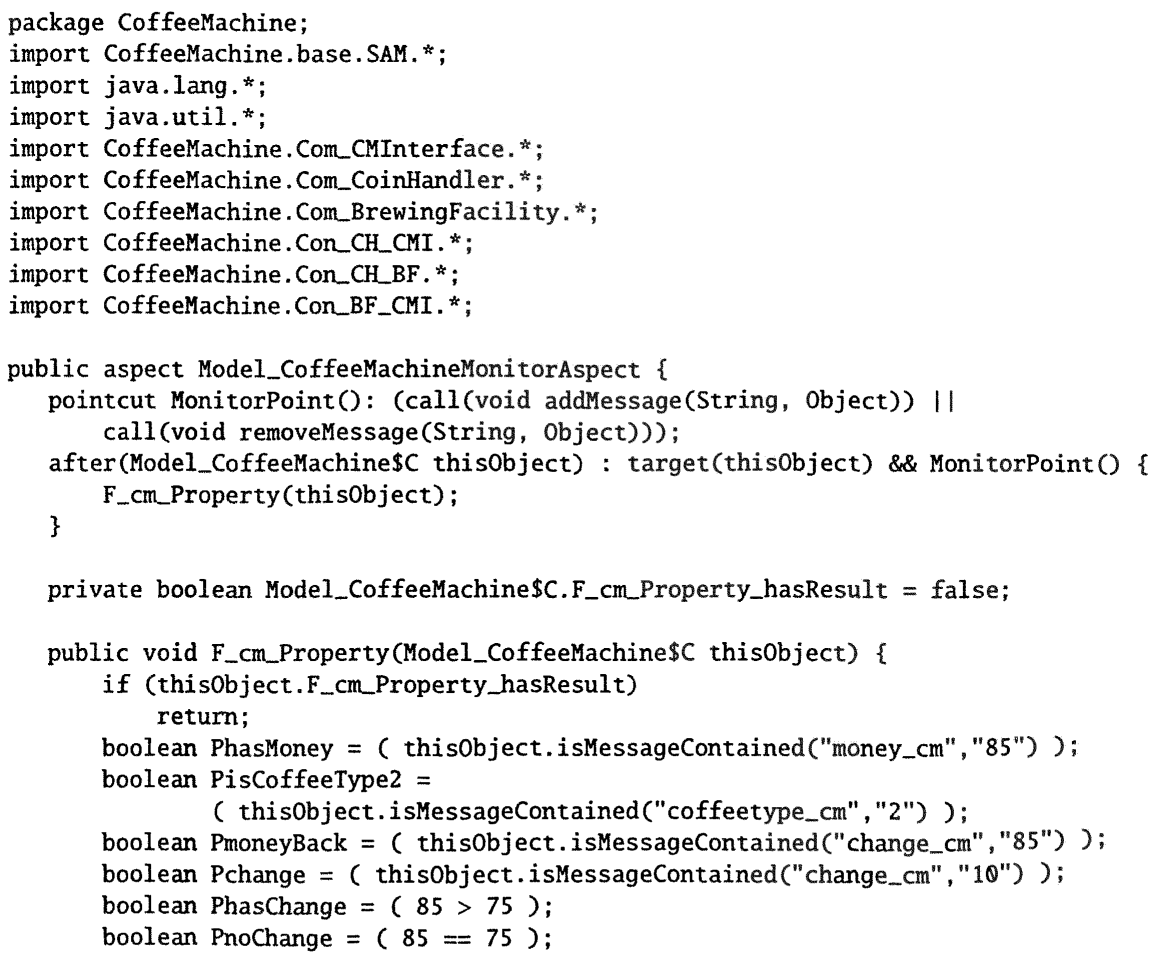


boolean PgetCoffee $=$ ( thisObject.isMessageContained ("ready_enjoy_cm", "1"));

switch(thisobject.F_cm_Property_\$state) \{

case 1:

thisObject.F_cm_Property_\$state $=$ PgetCoffee ? -1: PmoneyBack ? -1 :

PisCoffeeType2 ? PhasMoney ? $2:-1:-1$; break ;

case 2:

thisobject.F_cm_Property_\$state $=$ PgetCoffee ? -1: PmoneyBack ? -1: 2 ;

break ;

\}

if (thisobject.F_cm_Property_\$state $==-2$ ) \{

thisobject.F_cm_Property_hasResult = true;

System.out.println ("Formula F_cm_Property is violated!");

\} return;

if (thisobject.F_cm_Property_\$state $==-1$ ) \{

thisObject.F_cm_Property_hasResult = true;

System.out.println("Formula F_cm_Property holds!");

return;

\}

thisObject.F_cm_Property_hasResult $=$ false;

\}

System.out.println("Cannot judge Formula F...cm_Property currently!");

private int Model_CoffeeMachine $\$ C . F \_c m \_P r o p e r t y \_\$ s t a t e=1 ;$

pointcut ConstructorPoint (): (!within(SAMModel_Component) \&\&

execution(new(..)));

after(Model_CoffeeMachine $\$ C$ thisObject) :

target(thisObject) \&\& ConstructorPoint () \{

3 


\section{APPENDIX D \\ RUNTIME CODE FOR ONLINE SHOPPING}

The generated code for the online shopping contains hundreds of files, we cannot list every

file due to space limit. Following the ArchJava code for the component OnlineShopping.

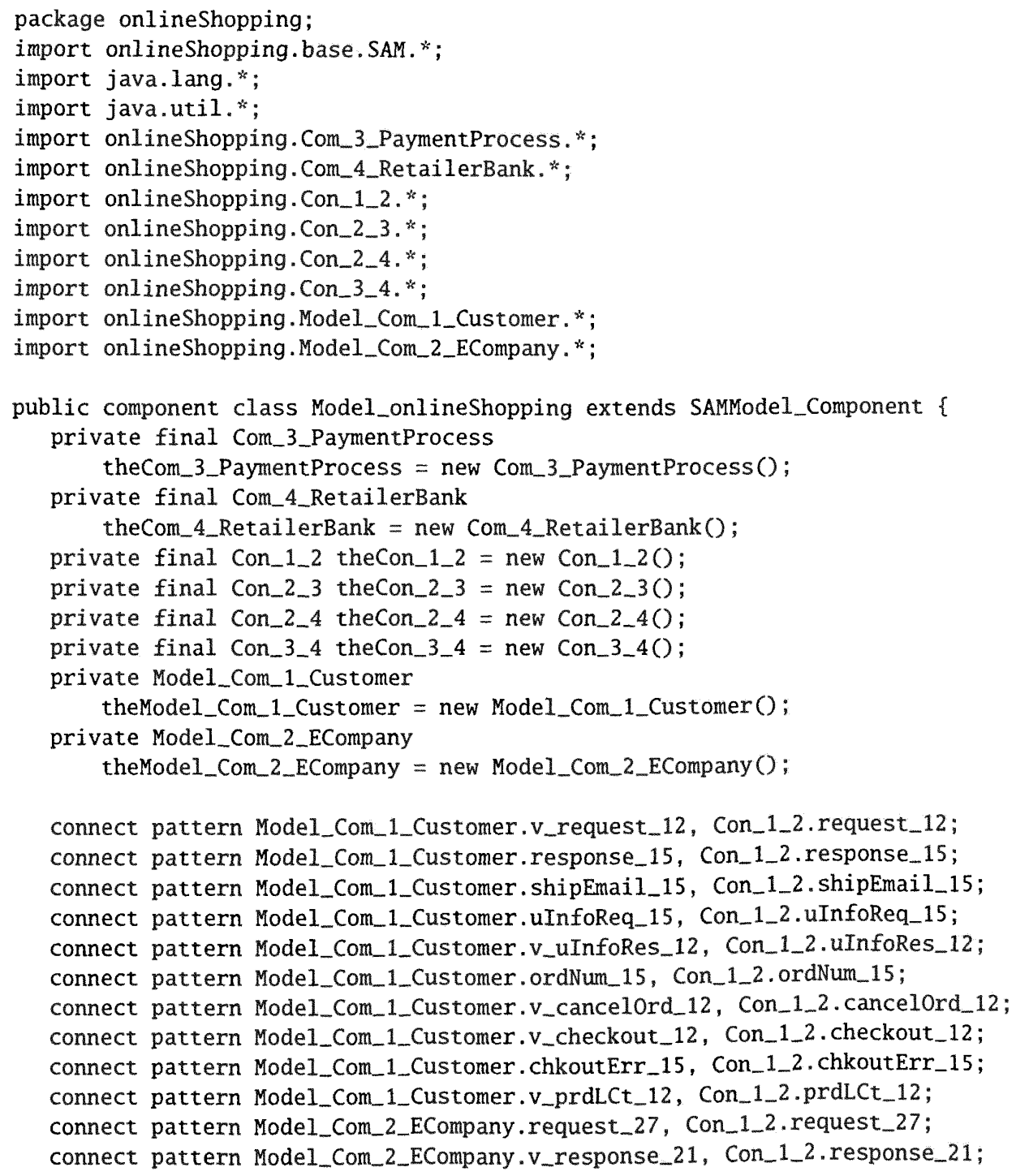


connect pattern Mode1_Com_2_ECompany.v_uInfoReq_21, Con_1_2.uInfoReq_21; connect pattern Model_Com_2_ECompany.uInfoRes_28, Con_1_2.uInfoRes_28; connect pattern Model_Com_2_ECompany.v_ordNum_21, Con_1_2.ordNum_21; connect pattern Model_Com_2_ECompany.cancelord_28, Con_1_2.cancelord_28; connect pattern Model_Com_2_ECompany.checkout_28, Con_1_2.checkout_28; connect pattern Model_Com_2_ECompany.checkout_27, Con_1_2.checkout_27; connect pattern Model_Com_2_ECompany.v_ccErr_21, Con_1_2.ccErr_21; connect pattern Model_Com_2_ECompany.prdLCt_27, Con_1_2.prdLCt_27; connect pattern Model_Com_2_ECompany.prdLCt_28, Con_1_2.prdLCt_28; connect pattern Model_Com_2_ECompany.v_ccAuth_23, Con_2_3.ccAuth_23; connect pattern Model_Com_2_ECompany.ccRes_28, Con_2_3.ccRes_28; connect pattern Model_Com_2_ECompany.v_backChBk_24, Con_2_4.backChBk_24; connect pattern Model_Com_2_ECompany.v_chargeRet_24, Con_2_4.chargeRet_24; connect pattern Com_3_PaymentProcess.ccAuth_3, Con_2_3.ccAuth_3; connect pattern Com_3_PaymentProcess.ccRes_32, Con_2_3.ccRes_32; connect pattern Com_3_PaymentProcess.backCh_3, Con_3_4.backCh_3; connect pattern Com_4_RetailerBank.backChBk_4, Con_2_4.backChBk_4; connect pattern Com_4_RetailerBank. chargeRet_4, Con_2_4.chargeRet_4; connect pattern Com_4_RetailerBank.backCh_43, Con_3_4.backCh_43;

public Model_onlineShopping ()\{ super ("onlineShopping", "'); connect (theModel_Com_1_Customer.v_request_12, theCon_1_2.request_12); connect (theModel_Com_1_Customer.response_15, theCon_1_2.response_15); connect (theModel_Com_1_Customer.shipEmail_15, theCon_1_2.shipEmail_15); connect (theModel_Com_1_Customer.uInfoReq_15, theCon_1_2.uInfoReq_15); connect (theModel_Com_1_Customer.v_uInfoRes_12, theCon_1_2.uInfoRes_12); connect (theModel_Com_1_Customer.ordNum_15, theCon_1_2.ordNum_15); connect (theModel_Com_1_Customer.v_cancelord_12, theCon_1_2.cancelord_12); connect (theModel_Com_1_Customer.v_checkout_12, theCon_1_2.checkout_12); connect (theModel_Com_1_Customer,chkoutErr_15, theCon_1_2.chkoutErr_15); connect (theModel_Com_1_Customer.v_prdLCt_12, theCon_1_2.prdLCt_12); connect (theModel_Com_2_ECompany.request_27, theCon_1_2.request_27); connect (theModel_Com_2_ECompany.v_response_21, theCon_1_2.response_21); connect (theModel_Com_2_ECompany.v_shipEmail_21, theCon_1_2.shipEmail_21); connect (theMode1_Com_2_ECompany.v_uInfoReq_21, theCon_1_2.uInfoReq_21); connect (theModel_Com_2_ECompany.uInfoRes_28, theCon_1_2.uInfoRes_28); connect (theModel_Com_2_ECompany.v_ordNum_21, theCon_1_2.ordNum_21); connect (theModel_Com_2_ECompany.cancelord_28, theCon_1_2.cancelord_28); connect (theModel_Com_2_ECompany.checkout_28, theCon_1_2.checkout_28); connect (theModel_Com_2_ECompany.checkout_27, theCon_1_2.checkout_27); connect (theModel_Com_2_ECompany.v_ccErr_21, theCon_1_2.ccErr_21); connect (theModel_Com_2_ECompany.prdLCt_27, theCon_1_2.prdLCt_27); connect (theModel_Com_2_ECompany.prdLCt_28, theCon_1_2.prdLCt_28); connect (thelodel_Com_2_ECompany.v_ccAuth_23, theCon_2_3.ccAuth_23); connect (theMode1_Com_2_ECompany.ccRes_28, theCon_2_3.ccRes_28); connect (theModel_Com_2_ECompany.v_backChBk_24, theCon_2_4.backChBk_24); connect (theModel_Com_2_ECompany.v_chargeRet_24, theCon_2_4.chargeRet_24); connect (theCom_3_PaymentProcess.ccAuth_3, theCon_2_3.ccAuth_3); connect (theCom_3_PaymentProcess.ccRes_32, theCon_2_3.ccRes_32); connect (theCom_3_PaymentProcess.backCh_3, theCon_3_4.backCh_3); connect (theCom_4_RetailerBank.backChBk_4, theCon_2_4.backChBk_4); connect (theCom_4_RetailerBank.chargeRet_4, theCon_2_4.chargeRet_4); connect (theCom_4_RetailerBank.backCh_43, theCon_3_4.backCh_43); subComponents . add (theCom_3_PaymentProcess); 


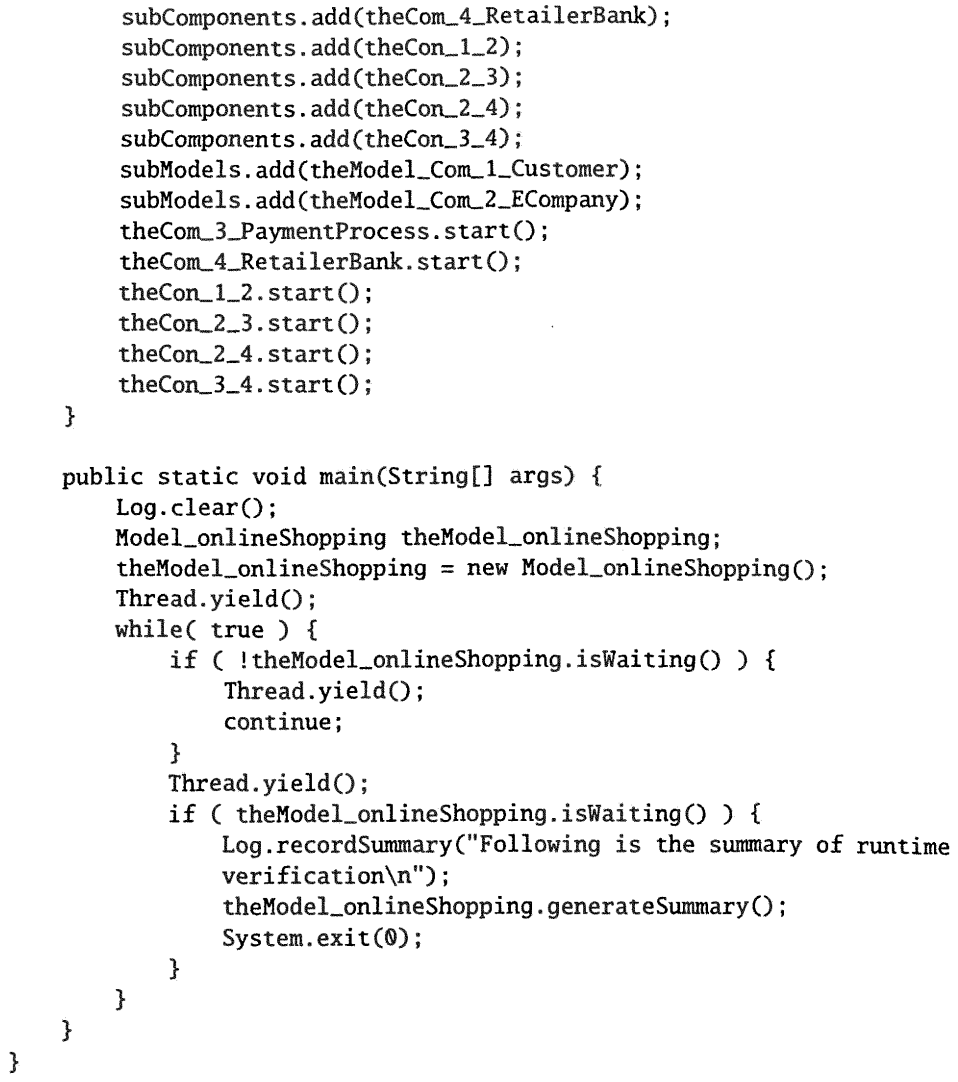

The generated aspectj code for the component ECompany is shown in the following.

public aspect Model_Com_2_ECompanyMonitorAspect \{

pointcut MonitorPoint (): (call(void addMessage(String, Object)) II

call (void removeMessage(String, object)));

after(Model_Com_2_ECompany $\$ C$ thisObject) : target(thisObject) \&\& MonitorPoint $O\{$ \}

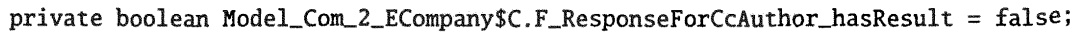

private boolean Model_Com_2_ECompany $\$ C . F \_R e s p o n s e F o r C C A u t h o r \_r e s u l t ~=$ false ;

public void F_ResponseForCCAuthor(Model_Com_2_ECompany $\$ C$ thisObject) \{

if (thisObject.F_ResponseForCcAuthor_hasResult)

return; 


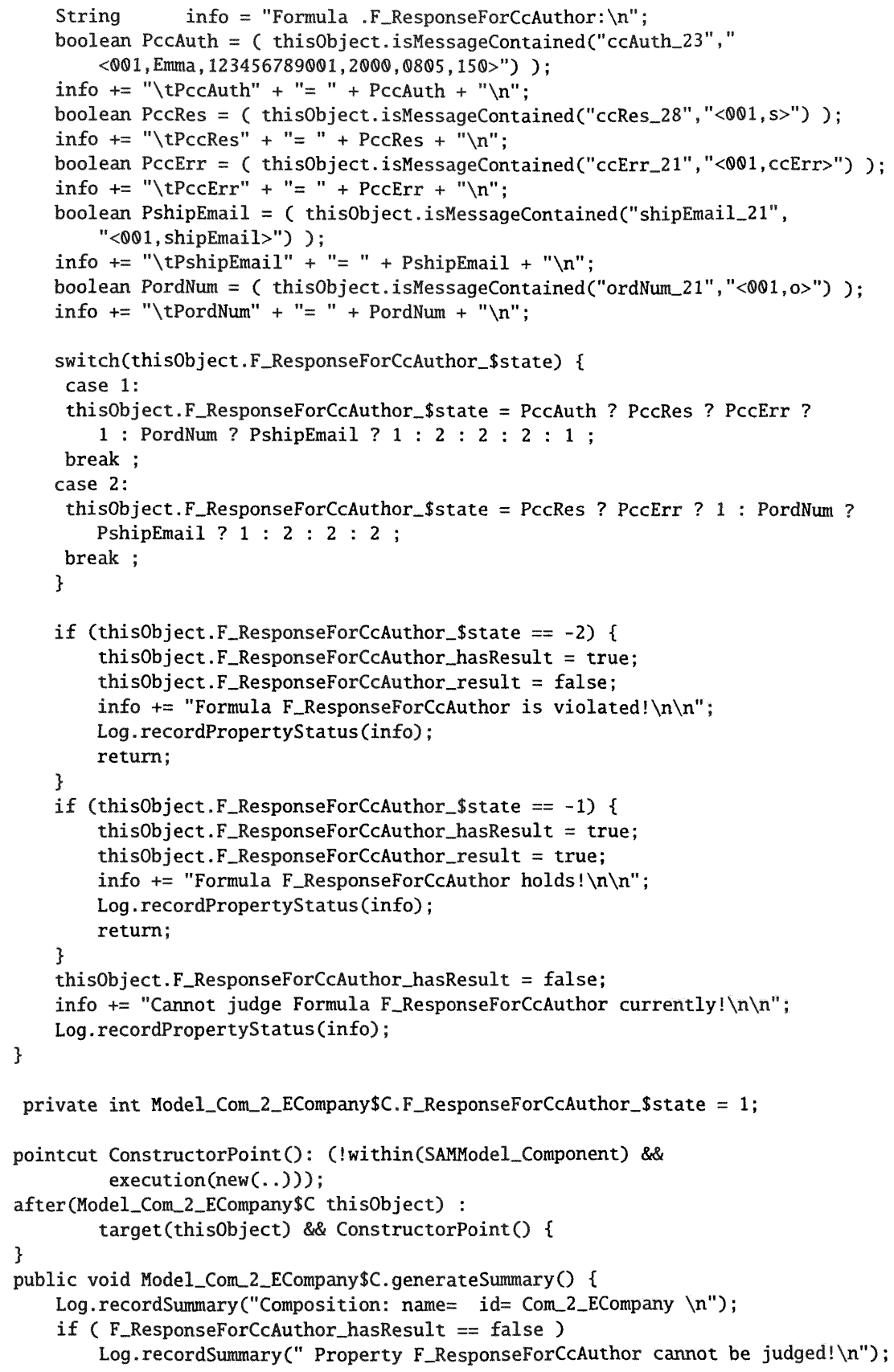


else \{

if (F_ResponseForCcAuthor_result) Log.recordSummary (" Property F_ResponseForCcAuthor held! $\backslash n "$ "); else

Log.recordSummary (" Property F_ResponseForCcAuthor failed! $\backslash n$ "); \}

super.generateSummary ();

\} \} 


\section{APPENDIX E \\ RUNTIME CODE FOR CRUISE CONTROL}

The generated code for the cruise control contains hundreds of files, we cannot list every file due to space limit. Following the Aspectj code for the component Cruising.

public aspect Com_Cruising_2MonitorAspect \{

pointcut MonitorPoint): (call(void addMessage(String, Object)) II

call(void removeMessage(String, Object)));

after(Com_Cruising_2\$C thisObject) : target(thisObject) \&\& MonitorPoint() \{

\}

F_Cruising_accl(thisobject);

private boolean Com_Cruising_2 $\$ C$.F_Cruising_accl_hasResult $=$ false;

private boolean Com_Cruising_2\$C.F_Cruising_accl_result = false;

public void F_Cruising_accl(Com_Cruising_2\$C thisobject) \{

if (thisobject.F_Cruising_accl_hasResult)

return;

String info = "Formula Cruising.F_Cruising_accl: $\backslash \mathbf{n}$ ";

boolean PP_C2_02 = ( thisObject.isMessageContained("C2_02", "<s1, 50, true, false $>$ ") );

info $+=$ "\tPP_C2_02" + "=" + PP_C2_o2 + "\n";

boolean PP_C2_i4 = ( thisObject.isMessageContained("C2_i4", "<s1, 50, true, false $>$ ") );

info $+=$ "\tPP_C2_i4" + "=" + PP_C2_i 4 + "\n";

switch(thisobject.F_Cruising_accl_\$state) \{

case 1:

thisObject.F_Cruising_accl_\$state $=$ PP_C2_i4 ? -1 : PP_C2_02 ? $2:-1 ;$ break ;

case 2:

thisObject.F_Cruising_accl_\$state $=$ PP_C2_i4 ?-1: 2 ;

break ;

\}

if (thisobject.F_Cruising_accl_\$state $==-2$ ) \{

thisObject.F_Cruising_accl_hasResult = true;

thisObject.F_Cruising_accl_result = false;

info $+=$ "Formula F_Cruising_accl is violated! $\backslash n \backslash n "$;

Log.recordPropertyStatus (info);

return;

\}

if (thisobject.F_Cruising_accl_\$state $==-1$ ) \{ 


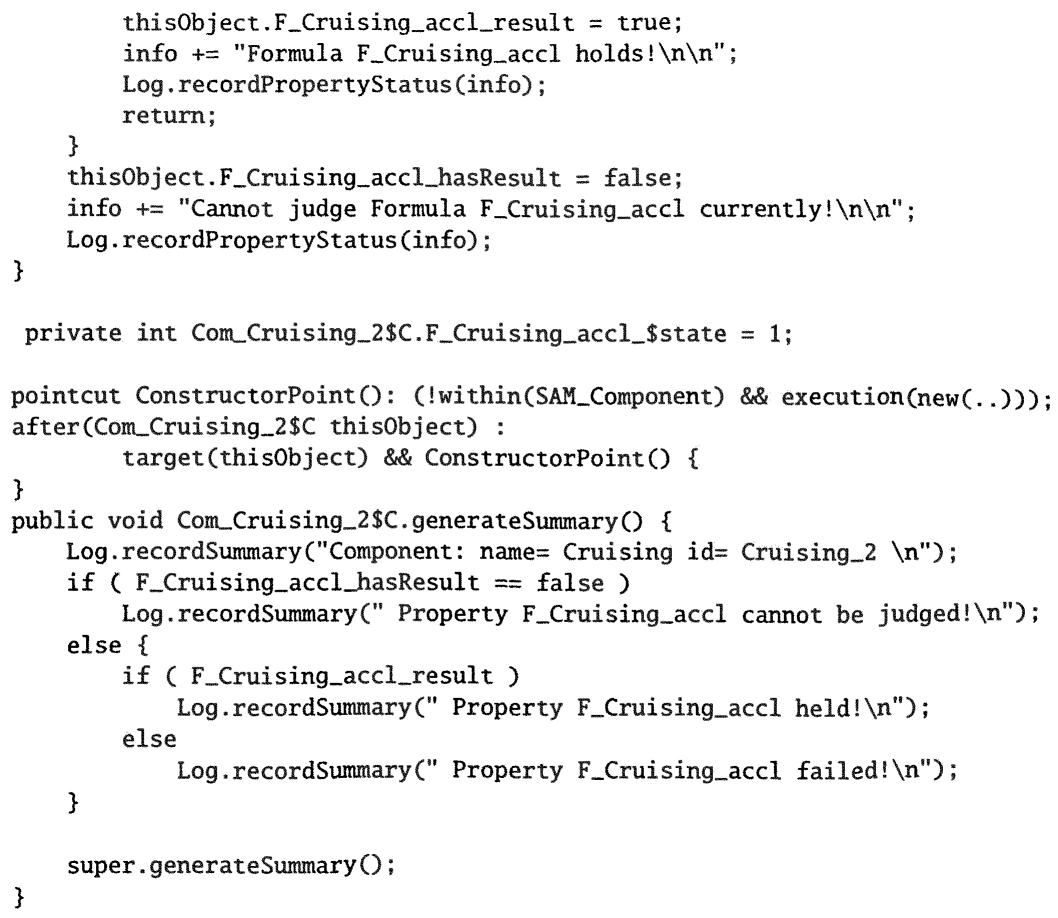




\section{APPENDIX F}

\section{SAM MODEL OF ONLINE SHOPPING SCENARIO}

Followings are the SAM model of online shopping scenario. The behavior model of each component and composition are shown in this appendix.

The first figure (Figure 33) is the whole structure of the SAM model of online shopping scenario, which was demonstrated in the Chapter 8. After that several components' behavior model, such as component User, Cart, Order, are demonstrated.

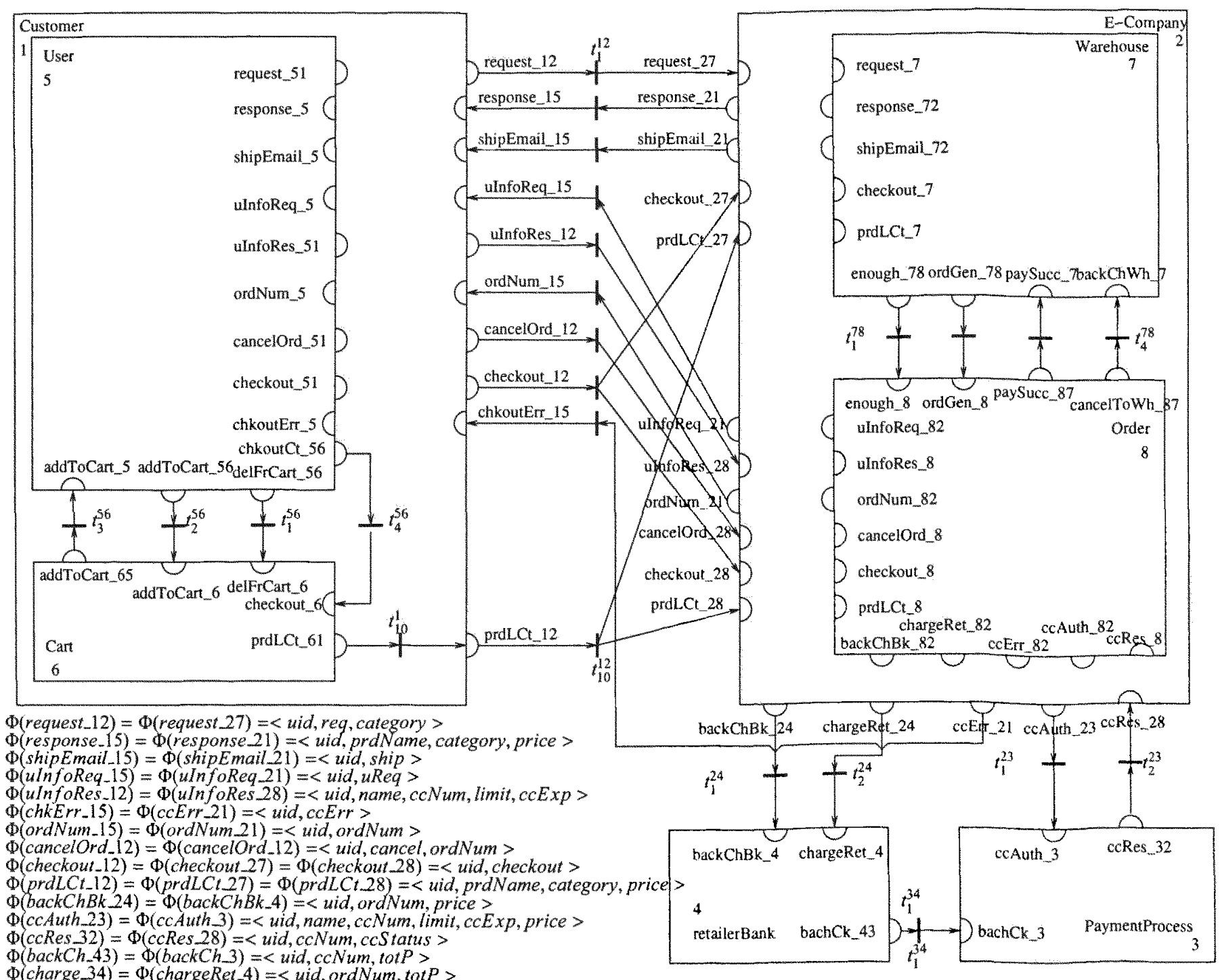

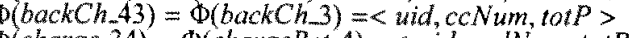

$\Phi($ charge 34$)=\Phi($ chargeRet 4$))=<$ uid, ordNum, tot $P>$

Figure 33: SAM model of online shopping 


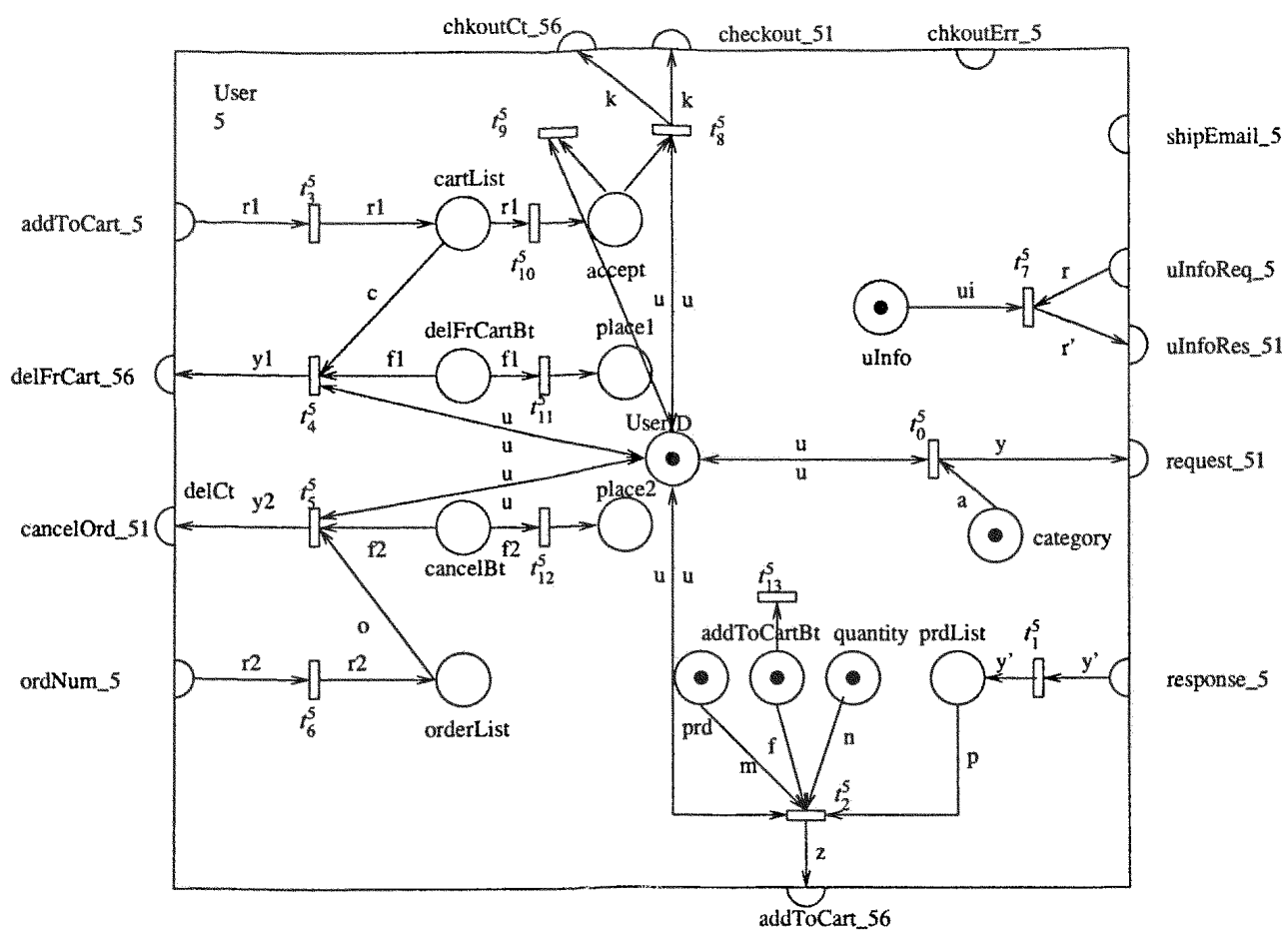

$\Phi(U \operatorname{ser} l D)=<u i d>$

$\Phi($ ordNum_5 $)=<$ uid, ordNum $>$

$\Phi($ cancelOrds 51$)=\langle$ uid, cancel, ordNum $>$

$\Phi($ uln $f o)=\langle$ name, $c c N u m$, cclimit, ccExp $>$

$\Phi($ delFrCart_56 $)=\langle$ uid, del, index $>$

$\Phi($ category $)=\langle$ categon $>$

$\Phi(p r d)=<$ name $>$

$\Phi($ add $T o$ Cart Bt $)=<$ addCart $>$

$\Phi(q u a n t i t y)=<$ int $>$

$\Phi($ prdList $)=<$ prdName, category, price $>$

$\Phi($ cartList $)=\langle$ uid, prdIndex $>$

$\Phi($ delFrCart $B t)=\langle$ index $>$

$\Phi($ cance $\mid B t)=\langle$ cancel $>$

$\Phi($ ordLisi $)=<$ uid, ordNum $>$

$\Phi($ accept $)=<$ uid, "checkout" $>$

$\Phi($ addToCart..5 $)=<$ uid, inde $x>$

$\Phi($ checkout_56) $=\Phi($ checkout 51$)=\langle$ uid, checkou $\rangle$

$\Phi($ chkoutErr.5) $=\langle$ uid, Siring $\rangle$

$\Phi($ ship Email $)=\langle$ uid, String $\rangle$

$\Phi($ uInforReq 5$)=\langle$ uid, uReq $>$

$\Phi($ ulnforRes 51$)=\langle$ uid, name, $c$ Num, limit, ccExp $>$

$\Phi($ request 51$)=\langle$ uid, Preq, cat $\rangle$

$\Phi($ response 5$)=<$ uid, prdName, category, price $>$

$\Phi($ place 1$)=\langle$ index $>$

$\Phi($ place 2$)=\langle$ index $>$

$\Phi($ addToCart .56$)=<$ uid, addCart, prdName, category, price, num $>$

$M_{0}($ UserID $)=($ uid $) \quad M_{0}($ uInfo $)=\{<$ name, ccNum, cclimit, ccExp $>\}$

$M_{0}($ category $)=\{$ category $\}$

$M_{0}($ prd $)=\{$ name $\}$

$M_{0}(a d d T o \operatorname{Cart} B t)=\{$ "addCart" $)$

$M_{0}($ quantity $)=\{$ quantity $\}$

$R\left(t_{0}^{5}\right)=\{y[1]=u \wedge y[2]=p \operatorname{Req} \wedge y[3]=a)$

$R\left(t_{1}^{5}, t_{3}^{5}, t_{6}^{5}, t_{9}^{5}, t_{10}^{5}, t_{11}^{5}, t_{12}^{5}\right)=\{$ true $\}$

$\left.R\left(t_{2}^{5}\right)=U=\operatorname{addCar} \wedge(\forall p \in P: p[1]==u \wedge p[2]=m \wedge z[1]=u \wedge z[2]=f \wedge z[3]=p[2] \wedge z[4]=p[3] \wedge z[5]=p[4] \wedge z[6]=n)\right\}$

$R\left(i_{5}^{5}\right)=\{f==$ cancel $\wedge \exists o \in O ; o[1]=z \wedge y[1]=u \wedge y 2[2]=f 2 \wedge y 2[3]=o[2] \wedge O=O-\{r 2\}\}$

$R\left(t_{4}^{5}\right)=\left\{f 1==\operatorname{del} \wedge \forall c \in C: c[1]==u \wedge y 1[1]=u \wedge y 1[2]=f 1 \wedge y 1[3]=c[2] \wedge C^{\prime}=C-\{c\}\right\}$

$R\left(r_{7}^{5}\right)=\left[r[1]==u \wedge r[2]==u \operatorname{Req} \wedge r^{\prime}[1]=u \wedge r^{\prime}[2]=u[1] \wedge r^{\prime}[3]=u i[2] \wedge r^{\prime}[4]=u\left[[3] \wedge r^{\prime}[5]=u[4]\right)\right.$

$R\left(s_{g}^{5}\right)=(h=$ checkout $\wedge k[1]=u \wedge k[2]=h \wedge O \neq n u l l]$

$R\left(t_{9}^{5}\right)=\{h=$ checkout $\wedge k[1]=u \wedge k[2]=h \wedge O \neq$ null $\}$

Figure 34: Behavior model of User component 


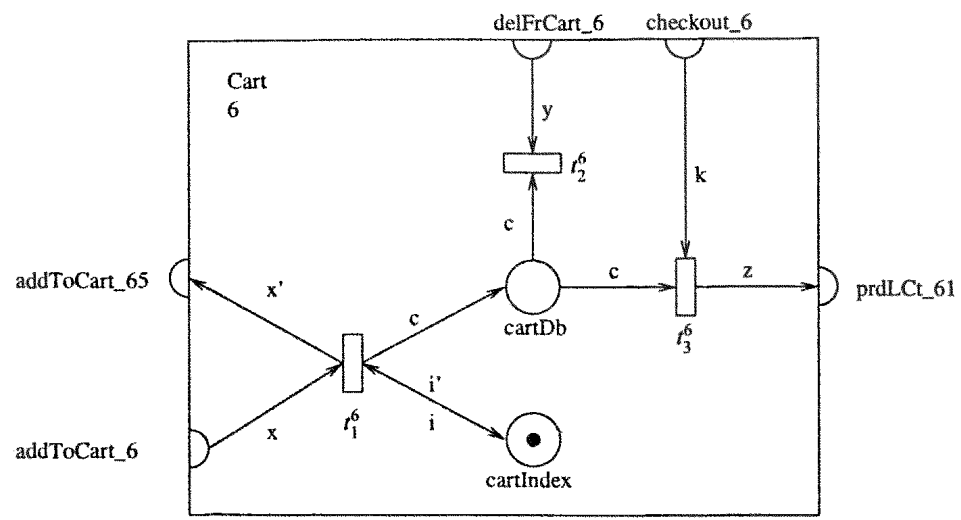

$\Phi($ addToCart_6 $)=<$ uid, addCart, prdName, category, price, quantity >

$\Phi($ addToCart_65) $=<$ uid, index $>$

$\Phi($ delFrCart_6) $=<$ uid, del, index $>$

$\Phi($ checkout_6 $)=\langle$ uid, checkout $>$

$\Phi($ prdLCt 61$)=<$ uid, index, prdName, category, price, quantity $>$

$\Phi($ cart $D b)=\langle$ uid, index, prdName, category, price, quantity $\rangle$

$\Phi($ cartIndex $)=<\operatorname{maxIndex}>$

$M_{0}($ cartindex $)=\{0\}$

$R\left(t_{1}^{6}\right)=(c[1]=x[1] \wedge c[2]=i \wedge c[3]=x[3] \wedge c[4]=x[4] \wedge c[5]=x[5] \wedge c[6]=x[6]$

$\left.\wedge x^{\prime}[1]=x[1] \wedge x^{\prime}[2]=i \wedge i^{\prime}=i+1\right\}$

$\left.R\left(\epsilon_{2}\right)=y[2]==\operatorname{del} \wedge \exists c \in C: c[2]==y[1] \wedge C^{\prime}=C-\{c\}\right\}$

$R\left(f_{3}^{6}\right)=\{k[2]==$ checkout $\wedge(\forall c \in C: k[1]==d[2]$

$\wedge z[1]=d[2] \wedge z[2]=d[3] \wedge z[3]=d[4] \wedge z[4]=d[5] \wedge z[5]=d[6] \wedge Z=Z \cup[z]\}$

\section{Figure 35: Behavior model of Cart component}

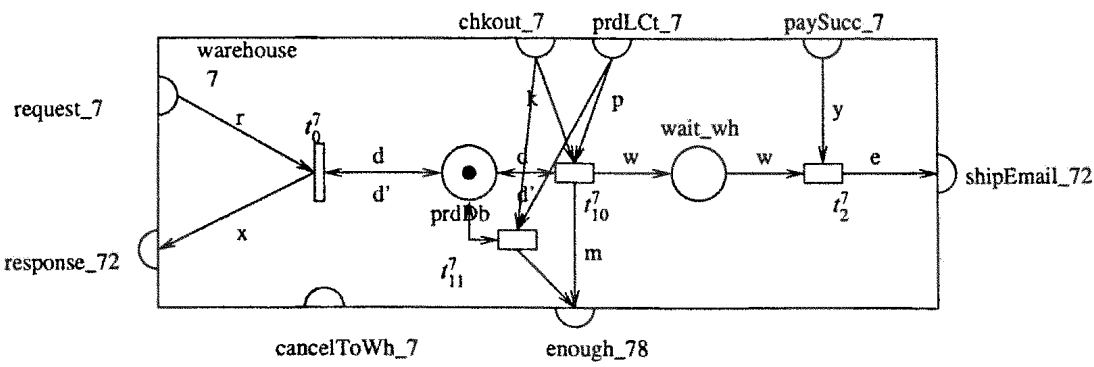

$\Phi($ prdDb $)=<$ prdName, category, price, quantity $>$

$\Phi($ wait_wh $)=\langle$ uid, wait $>$

$\Phi($ response_72) $=<$ uid, prdName, category, price $>$

$\Phi\left(\right.$ request $\left._{-} 7\right)=<$ uid, req, $_{\text {, category }}>$

$\Phi($ chkout_7 $)=\left\langle\right.$ uid, $^{\text {"kit }}$ checkout" $\rangle$

$\Phi($ prdList, 7$)=<$ uid, prdName, category, price, quantity $>$

$\Phi($ pays ucc_7 $)=\langle$ uid, payStatus >

$\Phi($ shipEmail 72$)=<$ uid, "ship" $>$

$\Phi($ enough_78) $=<$ uid, "enough" >

$\Phi($ cancelToWh.7) $=<$ uid, "cancel" >

$R\left(t_{0}^{7}\right)=[r[2]==p \operatorname{Req} \wedge r[3]==d[2] \wedge x[1]=r[1] \wedge x[2]=d[1] \wedge x[3]=d[2] \wedge x[4]=d[3])$

$R\left(t_{10}^{7}\right)=\{c[1]==p[1] \wedge c[2]==$ "checkout" $\wedge(d[2]==p[3] \wedge d[4]>=p[5] \wedge m[1]=p[1] \wedge m[2]=$ enough

$\wedge w[1]=p[1] \wedge w[2]=$ wait $\left.\left.\wedge d^{\prime}[4]=d[4]-p[5] \wedge d^{\prime}[1]=d[1] \wedge d^{\prime}[2]=d[2] \wedge d^{\prime}[3]=d[3]\right)\right]$

$R\left(t_{11}^{7}\right)=\left\{c[1]==p[1] \wedge c[2]==\right.$ "checkout" $^{\prime} \wedge(d[2]==p[3] \wedge d[4]>=p[5] \wedge m[1]=p[1] \wedge m[2]=$ "notenough"

$\wedge w[1]=p[1] \wedge w[2]=$ "wait" $\left.\left.\wedge d^{\prime}[4]=d[4] \wedge d^{\prime}[1]=d[1] \wedge d^{\prime}[2]=d[2] \wedge d^{\prime}[3]=d[3]\right)\right\}$

$R\left(t_{2}^{7}\right)=\{w[1]==y[1] \wedge y[2]==\operatorname{succ} \wedge e[1]=w[1] \wedge e[2]=$ shipEmail $]$

Figure 36: Behavior model of Warehouse component 


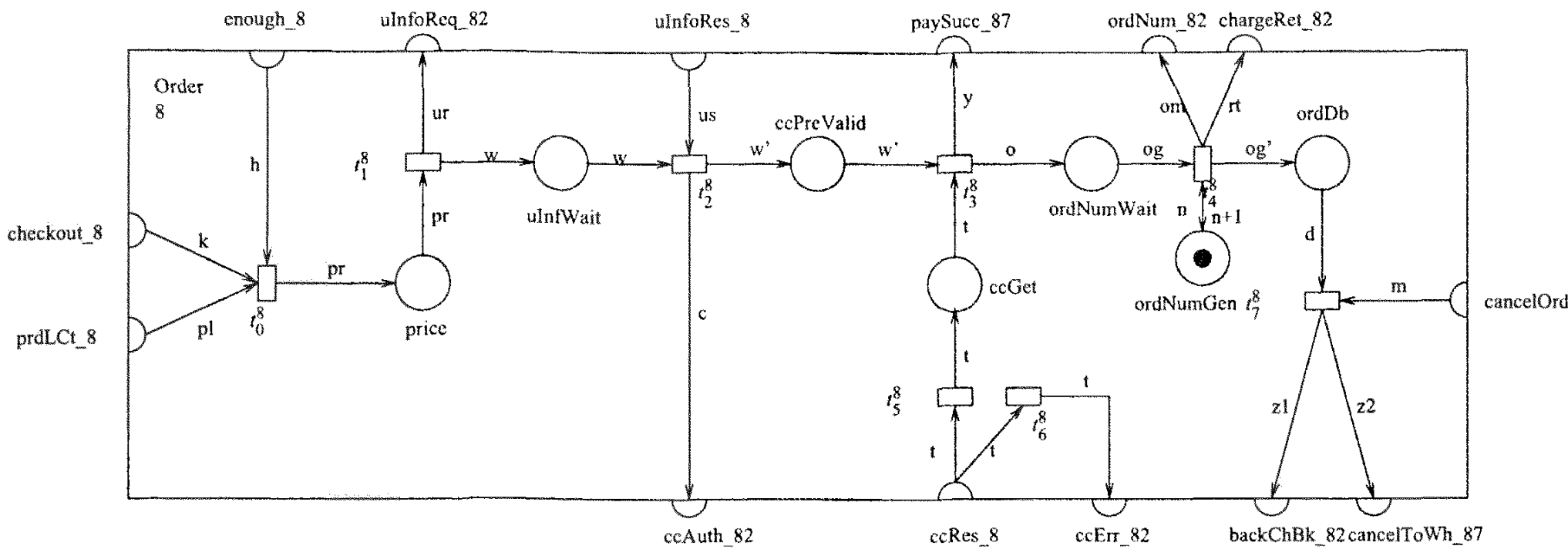

$\Phi($ prdLCt_8 $)=<$ idd, prdName, category, price, quantity >

$\Phi($ checkout 8$)=\langle$ uid, checkout $\rangle$

$\Phi($ enough_ 8$)=<$ uid, enough $>$

$\Phi($ wInfoReq_82) $=\langle$ uid, uReq $\rangle$

$\Phi($ ulnfoRes. 8$)=\langle$ uid, name, ccNum, limit, ccExp $>$

$\Phi($ ordGen 8$)=<$ wid, ordGen $>$

$\Phi($ paySucc.87 $)=<$ wid, paySucc $>$

$\Phi($ ordNum 82$)=\langle$ uid, ordNum $\rangle$

$\Phi($ cancelOrd 8$)=<$ id, cancel, ordNum $>$

$\Phi($ cancelToWh_87 $)=\langle$ uid, cancel, ord Num, tot $P>$

$\Phi($ backChBk_82) $=<$ uid, cancel, ordNum $>$

$\Phi\left(c c E r r_{-} 82\right)=\langle$ uid, $c c E r r\rangle$

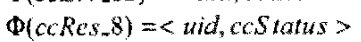

$\Phi($ ccAuth_82) $=\langle$ wid, name, ccNum, limit, ccExp, totP $\rangle$

$\Phi($ price $)=<$ uid, tor Price $>$

$\Phi($ un $/$ Wait $)=\langle$ uid, totPrice, wS taus $>$

$\Phi($ ccPreV alid $)=\langle$ uid, name, ccNum, linit, cc Exp, totPrice $\rangle$

$\Phi(\operatorname{cc} G e t) \approx\langle$ uid, $c c S u c c\rangle$

$\Phi($ ord $N u m G e n)=\langle$ uid, ord $N u m, 1 o t P>$

$\Phi($ ord Db $)=\langle$ uid, ord Num, tot $P>$

$\Phi($ chargeRet 82$)=\langle$ uid, ordNum, tol $P\rangle$

$R\left(t_{0}^{8}\right)=\left\{k[1]==p l[1]==h[1] \wedge h[2]==\operatorname{enough} \wedge p r[1]=k[1] \wedge \forall p^{\prime} \in p l: p r[2]=\Sigma\left(p^{\prime}[4] * p^{\prime}[5]\right)\right\}$

$R\left(t_{1}^{8}\right)=\{w[1]=\operatorname{pr}[1] \wedge w[2]=\operatorname{pr}[2] \wedge w[3]=$ wait $\wedge u[1]=z[1] \wedge u r[2]=u R e q\}$

$R\left(t_{2}^{8}\right)=\left[w[1]=u\left[[1] \wedge w^{\prime}[1]=w[1] \wedge w^{\prime}[2]=u s[2] \wedge w^{\prime}[3]=u s[3] \wedge w^{\prime}[4]=u s[4] \wedge w^{\prime}[5]=u[5] \wedge w^{\prime}[6]=w[2]\right\}\right.$

$R\left(t_{3}^{8}\right)=\left\{l[2]==c\right.$ Pass $\wedge w^{\prime}[1]==\left\{[1] \wedge y[1]=w^{\prime}[1] \wedge y[2]=\operatorname{poys} w \operatorname{cc} \wedge \operatorname{og}[1]=w^{\prime}[1] \wedge \operatorname{og}[2]=w^{\prime}[6]\right\}$

$R\left(t_{4}^{8}\right)=\left[o m[1]=\operatorname{og}[1] \wedge \operatorname{om}[2]=\operatorname{randomNum} \wedge \operatorname{og}^{\prime}[1]=\operatorname{og}[1] \wedge \operatorname{og}^{\prime}[2]=\operatorname{om}[2] \wedge \operatorname{og}^{\prime}[3]=\operatorname{og}[2] \wedge r[1]=\operatorname{og}[1] \wedge r t[2]=\operatorname{om}[2] \wedge r[3]=\operatorname{og}[2]\right]$

$R\left(t_{5}^{8}\right)=[\{[2]=$ "notpass" $\mid$

$R\left(t_{6}^{8}\right)=\left\{\left(r[2]={ }^{i z} \operatorname{ccs} u c c " \wedge \operatorname{cn}[1]=t[1] \wedge \operatorname{cn}[2]=t[2]\right)\right]$

$R\left(f_{7}^{8}\right)=\{m[2]==$ cancel $\wedge d[1]==m[1] \wedge d[2]==m[3] \wedge z[[1]=d[1] \wedge z[[2]=m[2] \wedge z 1[3]=m[3]$

$\wedge z 2[1]=d[1] \wedge z 2[2]=m[2] \wedge z 2[3]=m[3]) \wedge z 2[4]=d[3]\}$

Figure 37: Behavior model of Order component 


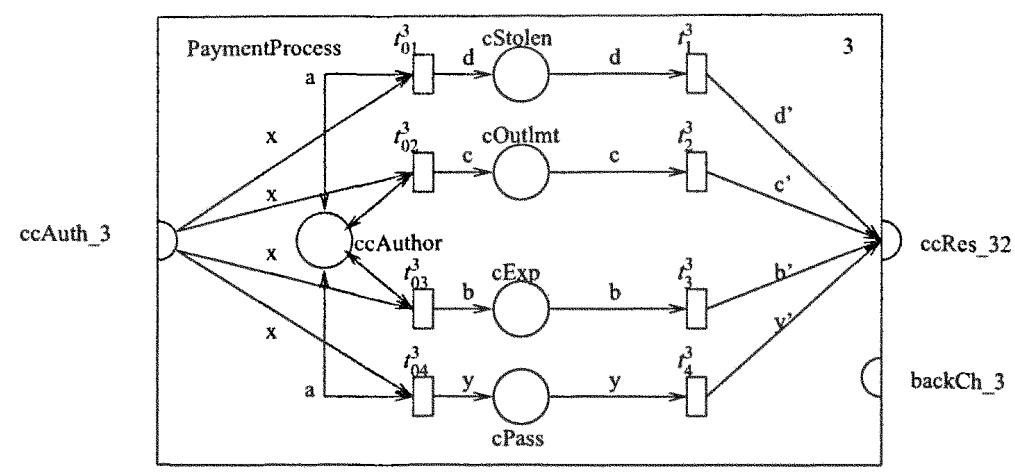

$\Phi($ ccAuth 3$)=\langle$ uid, name, ccNum, limit, ccExp, totP $\rangle$

$\Phi(c S$ tolen $)=<$ uid, $c c N$ um, stolen $>$

$\Phi($ ccRes 32) $=<$ uid, ccStatus $>$

$\Phi(c$ Outmi $)=\langle$ wid, $c c N u m$, outlimit $\rangle$

$\Phi($ backCh 3$)=\langle$ uid, cancel, tot $P\rangle$

$\Phi(c E x p)=<$ uid, ccNum, expire $>$

$\Phi(c c$ Author $)=<$ name, ccNum, limit, ccExp, totP, status $>$

$\Phi($ cPass $)=<$ uid, ceNum, true $>$

$R\left(t_{01}^{3}\right)=(\forall a \in A:(a[2]==x[3] \wedge a[1]==x[2] \wedge a[6]==$ stolen $\wedge d[1]=x[1] \wedge d[2]=x[2] \wedge d[3]=a[7])\}$

$R\left(t_{02}^{3}\right)=\{\forall a \in A:(a[2]==x[3] \wedge a[1]==x[2] \wedge a[3]<x[6] \wedge d[1]=x[1] \wedge d[2]=x[2] \wedge d[3]=$ "outlimit" $)\}$

$R\left(t_{03}^{3}\right)=(\forall a \in A:(a[2]==x[3] \wedge a[1]==x[2] \wedge a[4]<x[5] \wedge d[1]=x[1] \wedge d[2]=x[2] \wedge d[3]=$ "expire" $)\}$

$R\left(t_{04}^{3}\right)=\{\forall a \in A:(a[2]==x[3] \wedge a[1]==x[2] \wedge x[4]==a[3] \wedge x[4]>=x[6]\}$

$\wedge d[1]=x[1] \wedge d[2]=x[2] \wedge d[3]=$ irue $)$

$R\left(t_{1}^{3}\right)=\left\{d^{\prime}[1]=d[1] \wedge d^{\prime}[2]=d[3]\right\}$

$R\left(t_{2}^{3}\right)=\left\{c^{\prime}[1]=c[1] \wedge c^{\prime}[2]=c[3]\right\}$

$R\left(r_{3}^{3}\right)=\left\{b^{\prime}[1]=b[1] \wedge b^{\prime}[2]=b[3]\right\}$

$R\left(t_{4}^{3}\right)=\left\{y^{\prime}[1]=y[1] \wedge y^{\prime}[2]=y[3] \wedge z[1]=y[1] \wedge z[2]=p a y\right\}$

\section{Figure 38: Behavior model of PaymentProcess component}

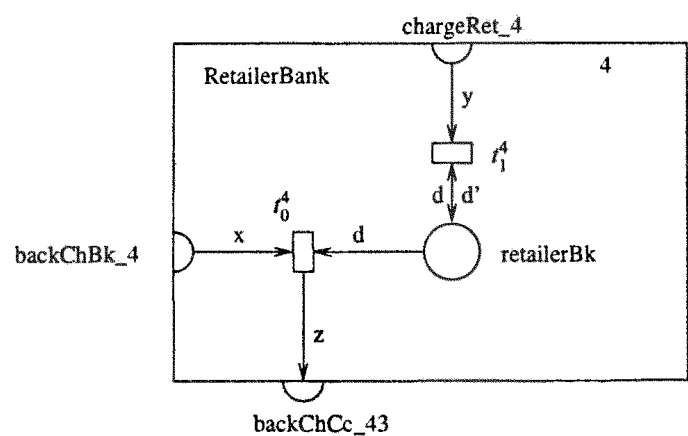

$\Phi\left(\right.$ backChet_-4 $_{-}=\langle$uid, ordNum, tot $P>$

$\Phi($ chargeRet_4 $)=\langle$ uid, ordNum, tot $P>$

$\Phi($ backChCc.43) $=\langle$ uid, ordNum, tot $P$, cancel $>$

$\Phi($ retailer $B k)=\langle$ uid, ordNum, tot $P>$

$R\left(t_{0}^{4}\right)=\left[\exists d \in D ; x[1]==d[1] \wedge x[2]==d[2] \wedge x[3]==d[3] \wedge D^{\prime}=D-(d)\right\}$

$R\left(t_{1}^{4}\right)=\left\{D^{\prime}=D \cup\{v\}\right]$

Figure 39: Behavior model of RetailerBank component 
Teaching Assistant

School of Computing and Information Sciences

Florida International University

Miami, Florida

1998-1999

Software Engineer

Everbright Securities, Ltd., Co.

Shenzhen, P.R.China

1994-1997

M.S., Electronic Science

NanKai University

TianJin, P.R.China

1992-1995

B.A., Foreign Language

TianJin Normal University

TianJin, P.R.China

\section{PUBLICATIONS AND PRESENTATIONS}

Y. Fu, Z. Dong, and X. He. A Method for Realizing Software Architecture Design. In Proceedings of QSIC06: Sixth International Conference on Quality Software. Beijing, China, October 26-28, 2006.

Y. Fu, Z. Dong, and X. He. Validating and Automating Composition of Web Services. In Proceedings of ICWE06: Sixth International Conference on Web Engineering, pages 217 -224, Palo Alto, CA, USA, July 10-14, 2006. (Best Paper Nomination)

Y. Fu, Z. Dong, and X. He. An Approach to Web Services Oriented Modeling and Validation. In Proceedings of ICSE06 workshop: The 2006 International Workshop on Service Oriented Software Engineering, Shanghai, China, May 27-28, 2006.

Y. Fu, Z. Dong, and X. He. An Approach to Validation of Software Architecture Model. In Proceedings of APSEC05: twelfth Asia-Pacific Software Engineering Conference (APSEC), December 15-17, 2005, TaiPei, Taiwan, China. 
Y. Fu, Z. Dong, and X. He. Formalizing and Validating UML Architecture Description of Web Systems. In Proceedings of ICWE06 workshop: Model-Driven Web Engineering (MDWE'06), Palo Alto, CA, USA, July 10-14, 2006.

Z. Dong, Y. Fu, and X. He. An Integrated Runtime Monitoring Framework for Software Architecture Model Verification. In Proceedings of The 9th IASTED International Conference on Software Engineering and Applications, Phoenix, AZ, USA, pages 106 - 111, November 14-16, 2005.

Y. Fu, Z. Dong, and X. He. A Methodology of Automated Realization of a Software Architecture Design. In Proceedings of 17th International Conference on Software Engineering and Knowledge Engineering, pages 412-417. Taipei, Taiwan, July 14-16, 2005.

Z. Dong, Y. Fu, and X. He. Automated Runtime Validation of Software Architecture Design. Lecture Notes in Computer Science, vol. 3816, pages 446 - 457. 2nd International Conference on Distributed Computing \& Internet Technology. Bhubaneswar, India. December $22-24,2005$.

Z. Dong, Y. Fu, and X. He. A Framework for Component-based System Modeling. In Proceedings of SEKE'06: The eighth International Conference on Software Engineering and Knowledge Engineering, pages 290 - 295, San Francisco Bay, CA, USA, July 5-7, 2006.

Z. Dong, Y. Fu, and X. He. Deriving Hierarchical Predicate/Transition Nets from Statechart Diagrams. In proceedings of the 15th International Conference on Software Engineering and Knowledge Engineering, pages $150-157$. San Francisco CA, U.S.A. July 1 3, 2003 .

Z. Dong, Y. Fu, and X. He. UML Consistency Checking Through Transformation based Petri Net Framework. The workshop of Consistency in Model Driven Engineering (Co$\mathrm{MoDE}$ ), in conjunction with European Conference on Model Driven Architecture, Nuremberg, Germany. November $7-10$ th, 2005. 